\title{
Visualization and quantification of tumour biology for personalized radiotherapy
}

Citation for published version (APA):

Even, A. J. G. (2017). Visualization and quantification of tumour biology for personalized radiotherapy. [Doctoral Thesis, Maastricht University]. Maastricht University. https://doi.org/10.26481/dis.20171220ae

Document status and date:

Published: 01/01/2017

DOI:

10.26481/dis.20171220ae

Document Version:

Publisher's PDF, also known as Version of record

\section{Please check the document version of this publication:}

- A submitted manuscript is the version of the article upon submission and before peer-review. There can be important differences between the submitted version and the official published version of record.

People interested in the research are advised to contact the author for the final version of the publication, or visit the DOI to the publisher's website.

- The final author version and the galley proof are versions of the publication after peer review.

- The final published version features the final layout of the paper including the volume, issue and page numbers.

Link to publication

\footnotetext{
General rights rights.

- You may freely distribute the URL identifying the publication in the public portal. please follow below link for the End User Agreement:

www.umlib.nl/taverne-license

Take down policy

If you believe that this document breaches copyright please contact us at:

repository@maastrichtuniversity.nl

providing details and we will investigate your claim.
}

Copyright and moral rights for the publications made accessible in the public portal are retained by the authors and/or other copyright owners and it is a condition of accessing publications that users recognise and abide by the legal requirements associated with these

- Users may download and print one copy of any publication from the public portal for the purpose of private study or research.

- You may not further distribute the material or use it for any profit-making activity or commercial gain

If the publication is distributed under the terms of Article $25 \mathrm{fa}$ of the Dutch Copyright Act, indicated by the "Taverne" license above, 


\section{Visualization and quantification of tumour biology for personalized radiotherapy}




\section{Cover}

The cover image shows a supervoxel representation of a lung cancer patient. The coloured hexagons symbolize different ways (different imaging modalities) of looking at the same underlying structure.

\section{Production}

Typesetting: This thesis was created using $\mathrm{ET}_{\mathrm{E}} \mathrm{X}$

Printing: $\quad$ Ipskamp Printing BV, Enschede

ISBN: $\quad$ 978-94-028-0857-5

\section{Copyrights}

(C) Copyright A.J.G. Even, Maastricht 2017

No parts of this publication may be reproduced in any form without prior written permission of the holder of the copyrights. 


\title{
Visualization and quantification of tumour biology for personalized radiotherapy
}

\author{
Proefschrift \\ ter verkrijging van de graad van doctor aan de Universiteit Maastricht, \\ op gezag van de Rector Magnificus Prof. dr. Rianne M. Letschert, \\ volgens het besluit van het College van Decanen, \\ in openbaar te verdedigen \\ op woensdag 20 december 2017 om 16:00 uur \\ door
}

Aniek Johanna Geertruida Even 


\section{Promotor}

Prof. dr. P. Lambin

\section{Co-promotor}

Dr. ir. W. van Elmpt

\section{Beoordelingscommissie}

Prof. dr. F.C.S. Ramaekers (voorzitter)

Prof. dr. ir. A.L.A.J. Dekker

Prof. dr. U.A. van der Heide (Leids Universitair Medisch Centrum; Nederlands Kanker Instituut) Prof. dr. D. Thorwarth (Eberhard Karls University, Tübingen, Duitsland)

Prof. dr. ir. FJ.W. Verhaegen 



\section{Contents}

$\begin{array}{ll}\text { Introduction } & 9\end{array}$

$\begin{array}{lll}\text { Chapter } 1 & \text { Introduction and outline } & 11\end{array}$

$\begin{array}{ll}\text { Non-invasive imaging of drug uptake } & 27\end{array}$

Chapter 2 PET imaging of Zirconium-89 labelled cetuximab: a phase I trial in patients with head and neck and lung cancer

Chapter 3 Quantitative assessment of Zirconium-89 labelled cetuximab using PET/CT imaging in patients with advanced head and neck cancer: a theragnostic approach

Hypoxia and DCE-CT imaging for outcome prediction and treatment adaptation

Chapter 4 Prognostic and predictive value of hypoxia HX4 PET and DCE-CT imaging in non-small cell lung cancer: results of a prospective window-of-opportunity clinical trial with nitroglycerin

Chapter 5 PET-based dose painting in non-small cell lung cancer: comparing uniform dose escalation with boosting hypoxic and metabolically active subvolumes 
Chapter 6 Predicting tumour hypoxia in non-small cell lung cancer by combining CT, FDG PET and dynamic contrast-enhanced CT

Chapter 7 Clustering of multiparametric functional imaging to identify highrisk subvolumes in non-small cell lung cancer

Discussion

Chapter 8 The promise of multiparametric imaging in oncology: how do we move forward?

Chapter 9 General discussion and future perspectives

Appendices

List of abbreviations

Summary

Samenvatting (Dutch summary)

Valorization addendum 205

Acknowledgments (Dankwoord) 209

Curriculum vitae 


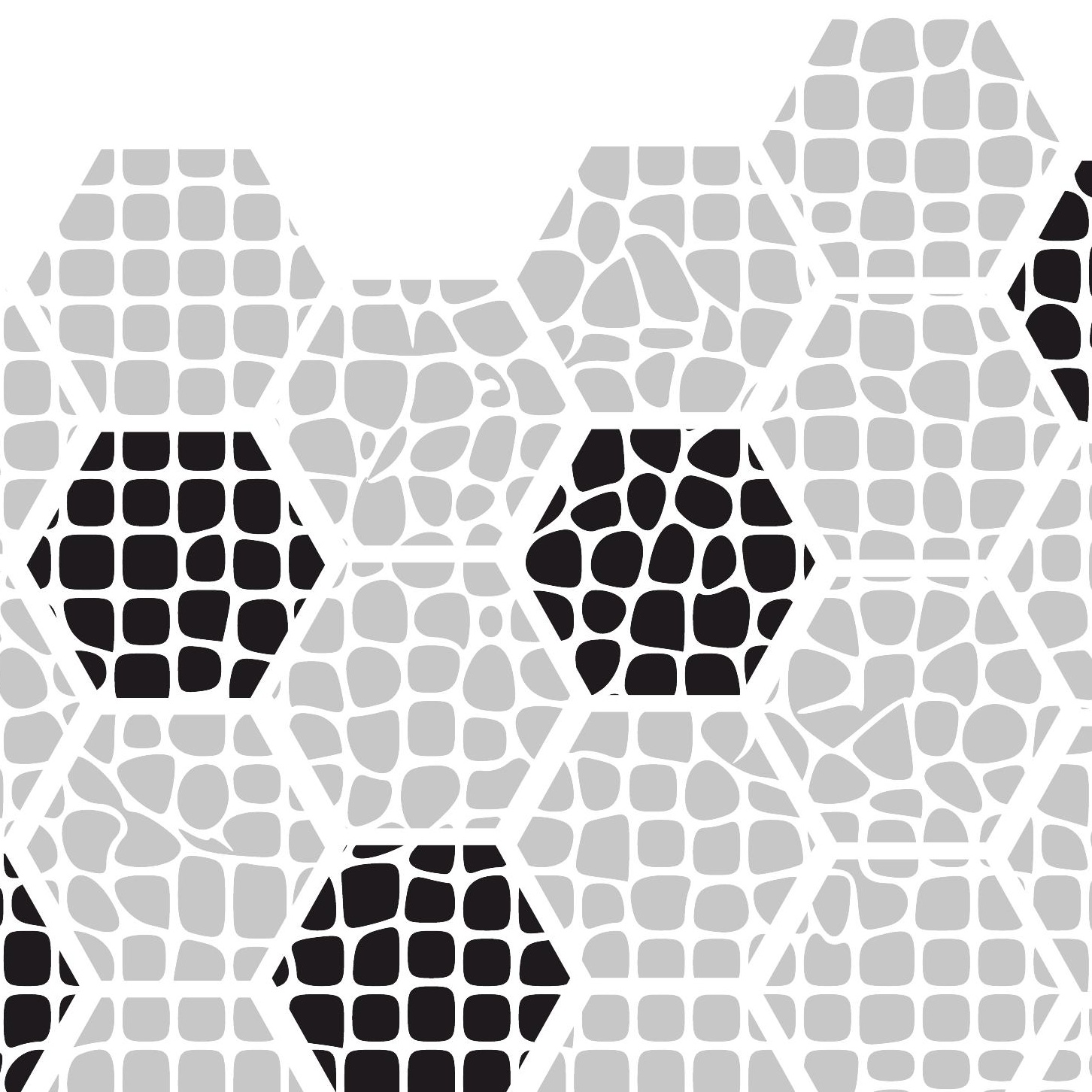


Introduction

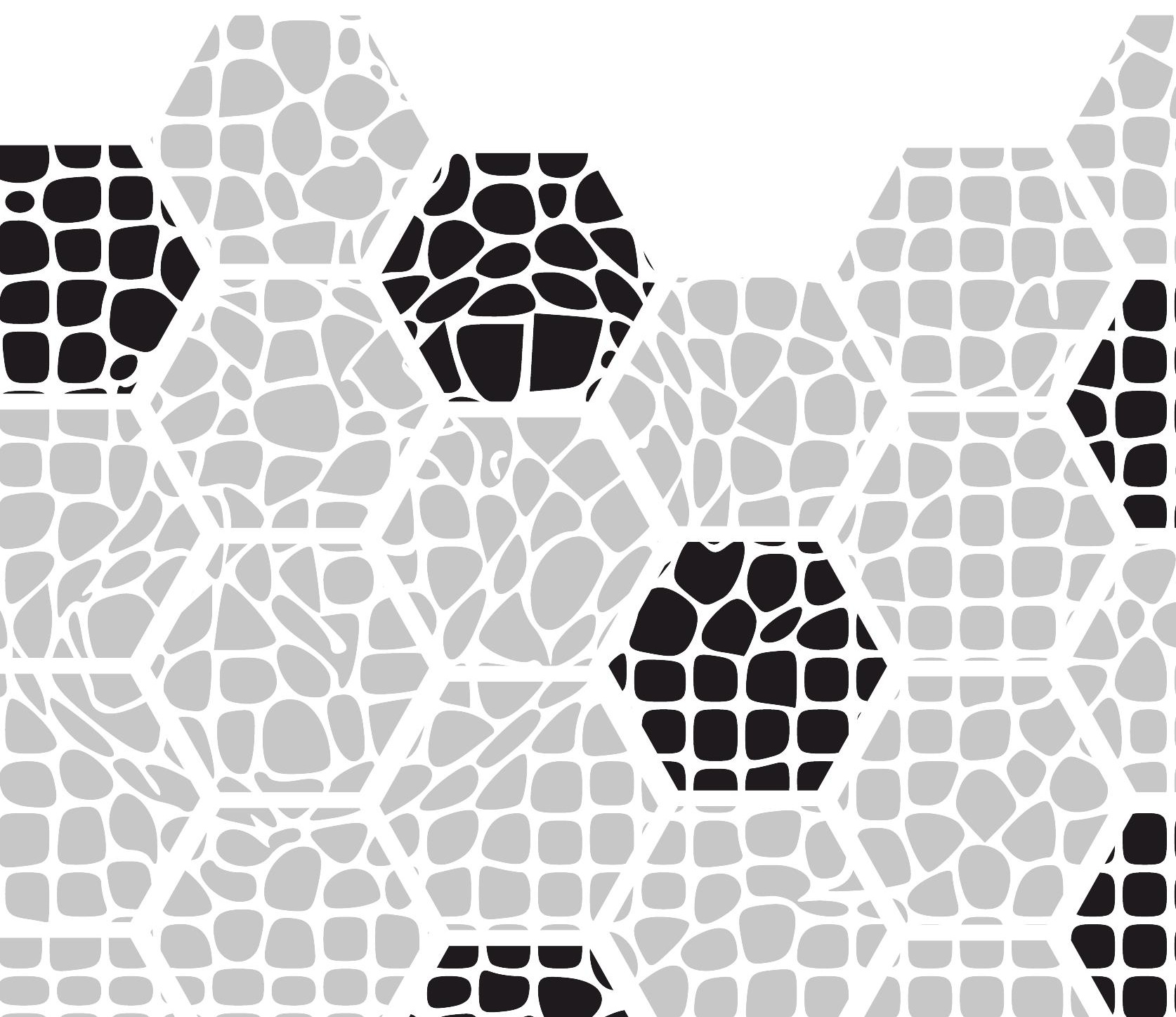




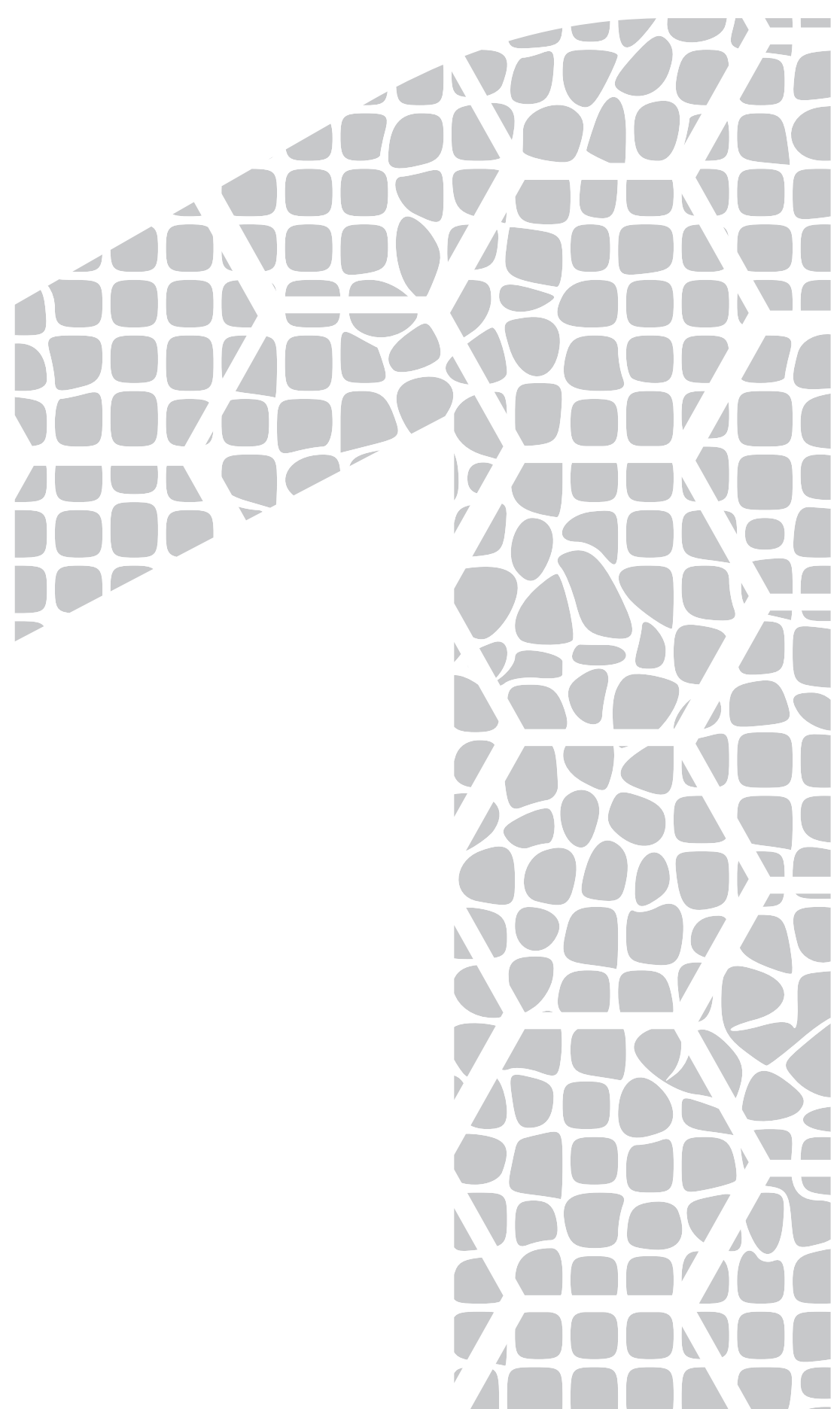




\section{Chapter 1}

\section{Introduction and outline}




\section{Cancer - a short introduction}

Cancer is the name of a group of diseases characterized by uncontrolled growth and spread into surrounding tissue. The first description of cancer dates back to an ancient Egyptian surgery textbook from about $1700 \mathrm{BC}$, which was probably a copy of a much older papyrus from 3000 to 2500 BC [1]. Although the Egyptians did not use the word cancer, the textbook described several cases of breast tumours that were removed with a "fire drill" [2, 3]. The Greek physician Hippocrates (460-370 BC) was the first one to refer to the disease as carcinos and carcinoma, which was later translated into cancer. Carcinos is the Greek word for crab, probably chosen due to the resemblance of the spreading of tumours to the legs and claws of a crab [3].

Nowadays, cancer is recognized as a worldwide health problem with over 14 million new cases in 2012. The number of new cancer cases is expected to increase and is estimated to reach almost 24 million by 2035 [4]. Even though the different treatment options have substantially improved over the past decades, survival for many cancer types remains poor, making cancer one of the leading causes of mortality and morbidity [4].

Cancer cells are mutated versions of our own cells. The mutations corrupt the regulatory systems in the cell and disturb processes such as development, cell growth and proliferation [5]. Despite the mutations, cancer cells are very similar to healthy cells and therefore a difficult target for anticancer therapies. Further complicating treatment is the fact that cancer is not one disease, but a collection of over 200 distinct cancer types [6]. Even for one type of cancer, genetic differences are found between patients (interpatient heterogeneity), within the primary tumour (intratumour heterogeneity), between metastases (intermetastatic heterogeneity), and within metastatic lesions (intrametastatic heterogeneity) [7].

\section{Cancer characteristics}

The diversity and complexity of cancer is believed to be a result of a number of underlying processes, referred to as "hallmarks of cancer". These hallmarks distinguish healthy cells from cancerous cells. The hallmarks are: (I) self-sufficiency in growth signals, (II) insensitivity to anti-growth signals, (III) tissue invasion and metastasis, (IV) limitless replicative potential, (V) sustained angiogenesis, (VI) evading apoptosis, (VII) avoiding immune destruction, (VIII) tumour-promoting inflammation, (IX) genome instability and mutation, and (X) reprogramming energy metabolism [8, 9]. Understanding the hallmarks of cancer can help improve the early detection of cancer, better develop targeted strategies, tailor treatments, and improve follow-up. Three hallmarks, crucial in the work presented in this thesis, will be discussed in more detail. 
The first hallmark, self-sufficiency in growth signals, is one of the most fundamental cancer characteristics. Normal cells require external signals to grow and divide. Cancers, however, can deregulate these signals and control their own growth. They can stimulate proliferation by producing growth factors, by activating normal cells to produce growth factors for them, by increasing the number of growth factor receptors, or by altering components downstream of the growth factor receptors [9]. An example of a growth factor receptor often overexpressed or mutated in human tumours is the epidermal growth factor receptor (EGFR) $[10,11]$. Elevated levels of EGFR have been correlated to a poor outcome in patients [12].

Sustained angiogenesis, another cancer hallmark, means that tumours can activate the formation of new blood vessels from pre-existing vessels. Tumours, like normal organs, require access to nutrients and oxygen, and need to be able to dispose of waste [13]. A developing tumour will first rely on diffusion for its nutrient supply, however, as soon as the tumour starts to grow beyond a size of $1-2 \mathrm{~mm}$, this will no longer be sufficient and blood vessels become essential for further growth [14]. Tumours activate angiogenesis to produce these new blood vessels. Angiogenesis is tightly regulated in adults and normally only temporarily enabled in processes such as wound healing. In contrast, almost all tumours activate angiogenesis and sustain its activation [9]. The imbalance in tumours between pro- and anti-angiogenic signalling creates a highly irregular and abnormal vasculature, with a heterogeneous blood flow. The vessels are dilated, leaky, tortuous and interconnected, and often fail to supply enough oxygen throughout the tumour $[14,15]$. Regions that are deprived of oxygen as a consequence, also referred to as tumour hypoxia, are more treatment resistant, and stimulate a more aggressive and invasive tumour type [16, 17].

Finally, tumours have the ability to reprogram their energy metabolism. In order to support the uncontrolled proliferation of tumour cells, adjustments of the energy metabolism are required [9]. In normal cells, energy is obtained by first processing glucose via an oxygen independent process, called glycolysis, followed by an oxygen dependent pathway, called oxidative phosphorylation. In hypoxic regions, tumours depend on the less energy efficient glycolysis for their energy supply. Even in the presence of oxygen, tumours cells will often resort to this oxygen independent pathway, referred to as the Warburg effect [18]. To compensate for the less energy efficient metabolism, tumours will upregulate glucose transporters to increase the import of glucose into the cytoplasm [9].

\section{Lung cancer and head and neck cancer}

The two types of cancer studied in this thesis are lung cancer, and head and neck cancer. Lung cancer is the most common cause of cancer related deaths worldwide [4]. 
The patient prognosis is highly depending on how advanced the disease is. Patients with locally advanced lung cancer have a five-year survival rate of only 15 to $20 \%$ [19]. Lung cancers can be subdivided in non-small cell lung cancer (around $85 \%$ of all lung tumours), and small cell lung cancer (around $15 \%$ ). Non-small cell lung cancers (NSCLC) are further divided in three major subtypes: squamous-cell carcinoma, adenocarcinoma, and large-cell lung cancer [20]. The vast majority of lung cancer cases are linked to tobacco smoking [21].

Head and neck cancers (HNC) are cancers that arise from the mouth, nose, throat, larynx or salivary glands. The majority of the cancers, about $95 \%$, begins in the squamous cells that line mucosal surfaces, the so-called head and neck squamous cell carcinoma (HNSCC) [22]. Treatment is often complex due to the proximity of the tumour to vital organs that provide breathing, swallowing, and speech. Tobacco and alcohol use are the most important risk factors for HNC [23].

\section{Treatment of cancer}

Cancer treatment options highly depend on the type of cancer, the advancement of the disease, and patient related factors. The most common treatments are surgery, chemotherapy, and radiotherapy. Surgery aims to physically remove the tumour; chemotherapy targets rapidly dividing cells with systemic drugs; radiotherapy uses ionizing radiation to kill cancer cells. Other treatment options include targeted therapy, immunotherapy and hormone therapy. Often a combination of these therapies is used to optimize treatment outcomes.

It is estimated that roughly $75 \%$ of all lung cancers and head and neck squamouscell carcinomas will benefit from radiotherapy as part of their treatment [24]. In patients with early stage NSCLC, surgery is the first treatment of choice [25]. However, if patients have contraindications to surgical resection, high dose radiotherapy is prescribed [19]. Most of the NSCLC patients will not have an early stage cancer, but will present with advanced disease at the time of diagnosis [20]. These patients, if not suitable for surgery, normally receive radiotherapy combined with chemotherapy, or only chemotherapy if the disease is metastasized.

Treatment approaches in head and neck vary depending on the tumour site. For early disease (about one third of the patients) both surgery and radiotherapy are viable options. In locally advanced HNSCC, radiotherapy is combined with chemotherapy, with or without surgery $[23,26]$. In HNC targeted therapies are also being investigated, focussing mostly on specific targeting of EGFR. The monoclonal antibody cetuximab is an example of such a targeted therapy approved for treatment in HNC. Monoclonal antibodies (mAb), i.e. antibodies that originate from one unique parent cell and are identical, specifically bind to antigens on the tumour surface, for example EGFR [27]. 


\section{Medical imaging in oncology}

Medical imaging has a vital role in the modern management of cancer. Medical imaging encompasses a wide range of non-invasive or minimally invasive modalities that provide a visual representation of the human body. Imaging is fundamental for cancer screening, diagnosis, staging, treatment, and response monitoring [28]. Radiotherapy heavily relies on medical imaging for treatment planning, patient positioning, and treatment verification [29]. After therapy, follow-up scans are performed routinely with different imaging techniques.

Medical imaging modalities can be roughly divided in two categories: techniques that visualize anatomy and techniques that assess functional or biological properties [30]. Magnetic resonance imaging (MRI) and computed tomography (CT) both provide anatomical information. CT is based on X-rays, while MRI makes use of the behaviour of nuclei in a magnetic field. Both techniques are commonly used to study the tumour size and morphology, and the location of the tumour relative to other organs. By adding contrast media or by adapting scanning protocols, both modalities can also provide functional information. Other modalities that provide functional information are positron emission tomography (PET) and single-photon emission computed tomography (SPECT) techniques. PET and dynamic contrast-enhanced CT imaging will be described in more detail in the next paragraphs.

\section{Positron emission tomography}

Positron emission tomography is a nuclear imaging modality based on positron emitting radionuclides [31]. The radionuclides are linked to biologically active molecules or drugs that act as carriers. These radionuclide labelled compounds, i.e. PET tracers, are injected in the body, accumulate in specific regions over time depending on the tracer, all the while releasing positrons. The positrons react in the body with nearby free electrons and in this annihilation reaction, a pair of gamma photons are released, travelling in opposite directions. The gamma photons are measured with a ring of detectors around the patient. The line in which the photons travel can be used to reconstruct the origin of the signal, to determine the location of the accumulated PET tracer (e.g. inside the tumour). A schematic drawing of PET imaging is shown in Figure 1.1. PET is commonly combined with CT, or more recently with MRI, to provide both functional and anatomical information in 3D in one imaging session. 


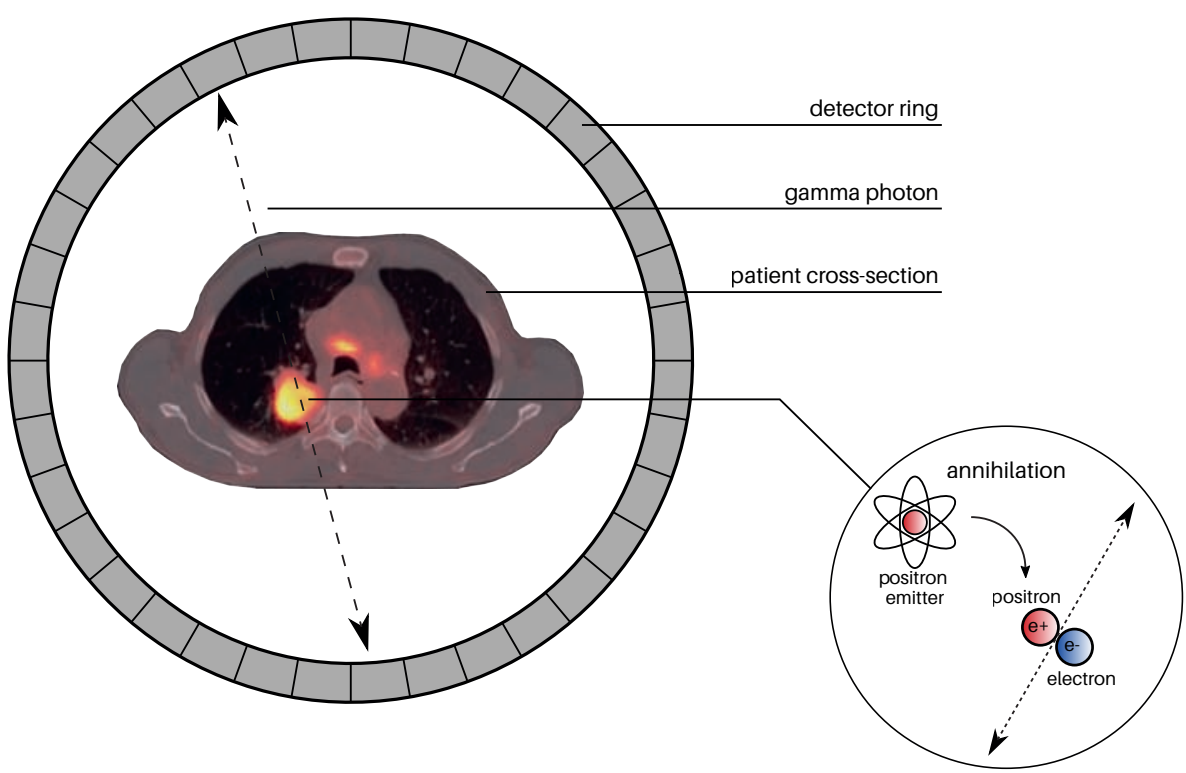

Figure 1.1. Schematic drawing of a patient in a PET scanner. The positron-emitting radionuclide has accumulated in various body tissues after some time. The higher concentration leads to more gamma photons being emitted from those regions and so the map of activity can be reconstructed.

\section{Selecting PET tracers to visualize different biological processes}

Depending on the PET tracers used, different biological processes can be visualized. By far the most widely used PET tracer, and the one most frequently used in the clinical workflow, is fluorodeoxyglucose $\left({ }^{18} \mathrm{~F}-\mathrm{FDG}\right)$, a glucose analog labelled with the radionuclide Fluorine-18. Just like glucose, FDG will be actively transported to cells with a high glucose demand. However, unlike glucose, FDG cannot undergo glycolysis and will get trapped in the cell after phosphorylation [32]. Generally, tumours have an upregulated glucose metabolism, as discussed previously as one of the hallmarks of cancer. As a consequence of this high glucose metabolism, a high accumulation of FDG is found in tumours. A disadvantage of FDG PET is that it is not tumour specific; also normal tissues with a high glucose metabolism or inflamed tissue will show up on an FDG PET scan.

Another tumour characteristic that can be visualized using PET is tumour hypoxia. Hypoxia, as described before, caused by the rapid tumour growth in combination with an underdeveloped vasculature, has been related to a more aggressive and treatment resistant tumour phenotype. The majority of hypoxia PET tracers are based on nitroimidazoles. Nitroimidazoles passively diffuse through a cell membrane where 
they are reduced into reactive radicals. This process is reversible in the presence of oxygen; the tracer is immediately reoxidized and can freely flow out of the cell again. In the absence of oxygen however, the radicals are further reduced, react with macromolecules in the cell, and get trapped [33]. ${ }^{18} \mathrm{~F}$-MISO, ${ }^{18} \mathrm{~F}$-FAZA and ${ }^{18} \mathrm{~F}-\mathrm{HX} 4$ are some examples of nitroimidazole-based PET tracers [34].

A third promising group of PET tracers is based on monoclonal antibodies (mAb). Monoclonal antibodies, e.g. cetuximab (introduced above), can specifically bind to antigens on the tumour surface. This enhances the immune system to attack the tumour cells, or targets tumour growth factor receptors, to restrict tumour proliferation. By labelling the mAb with positron-emitting radionuclides, a technique called immuno-PET [35], the expression of the antigens can be quantified and the efficiency of the $\mathrm{mAb}$ treatment can be assessed. The binding process of the mAb to the antigens is relatively slow, hence a long lasting positron emitting radionuclide is needed with a long half-life, for example Zirconium-89 $\left({ }^{89} \mathrm{Zr}\right)$ or Iodine-124 $\left({ }^{124} \mathrm{I}\right)$ [36].

\section{Dynamic contrast-enhanced CT}

Dynamic contrast-enhanced CT (DCE-CT) is a technique that dynamically images the flow of contrast material in a tumour to gain insight into the tumour vasculature [37]. First, a baseline CT image is acquired without any contrast material. Next, a bolus of iodinated contrast agent is injected intravenously and a series of CT images is acquired of the tumour region. The changes in enhancement on the $\mathrm{CT}$ are directly proportional to the concentration of contrast material [37]. This enhancement over time is used to construct time-attenuation curves, as shown in the box in Figure 1.2.

DCE-CT is used to extract several physiological parameters related to the tumour vasculature. In the first 45-60 seconds after injection of the contrast agent, i.e. first pass, the majority of the contrast agent is intravascular [38]. This phase can be used to assess the perfusion of the tumour, i.e. blood flow, and the blood volume. After the first phase, the contrast agent starts passing into the extravascular space. The amount of contrast agent in the extravascular space will increase over time until a balance is reached between the contrast material passing from the intravascular space to the extravascular space and from the extravascular space back into the vasculature. To acquire knowledge about vascular permeability, the DCE-CT scans should include images acquired around two to ten minutes after injection that will show this transition from the vascular to the extravascular space and back [38, 39]. 


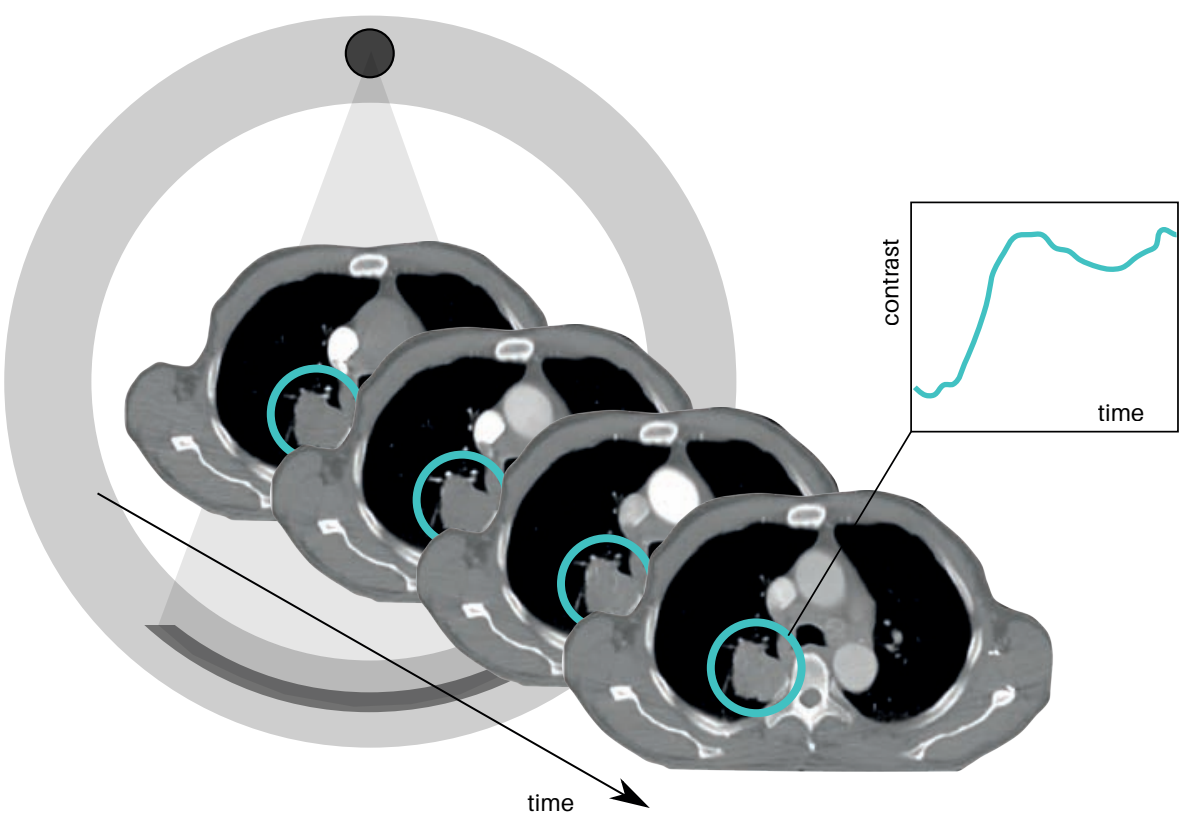

Figure 1.2. Dynamic-contrast enhanced CT image acquisition. A bolus of contrast material is injected in the patient. The distribution of this contrast is followed by acquiring a series of CT scans, centred around the tumour. The resulting time-attenuation curve (displayed in the top right box) can be used to model tumour vasculature.

\section{Functional imaging for personalized radiotherapy}

The main goal of personalized therapy is to tailor treatment to improve patient outcome and/or reduce treatment related toxicities [40]. Functional imaging in radiotherapy has the potential to significantly improve staging of cancer, targeting of radiotherapy, stratification of patients, and prediction and monitoring of therapeutic efficacy and toxicity [41]. Tumour stage and functional imaging characteristics can help selecting the most beneficial treatment for a certain patient. For example, functional imaging might predict if there is benefit of adding targeted therapies to radiotherapy. In patients where no benefit is expected, the targeted therapy will not be prescribed and the patients are not exposed to any unnecessary additional treatment induced toxicities.

One major challenge in radiotherapy is the definition of the target volume. Modern irradiation techniques allow very precise and conformal deposition of radiation dose in a target. The more precise this delivery is, the more important it becomes that all tumour lesions are correctly identified. FDG PET imaging is widely used to aid target delineation. Adding this functional imaging modality has shown to improve 
target definition, over anatomical imaging alone, in several tumour types [42, 43]. Functional imaging also allows integrating intratumour heterogeneities into treatment planning. Traditionally, radiotherapy doses are delivered uniformly throughout the whole tumour. However, since tumour are radiobiologically heterogeneous, not all tumour regions will achieve the same cell damage with the same radiation dose. Hence, if the spatial radioresistance information is known, the radiation dose can be redistributed inside the tumour based on this information acquired from functional imaging. This spatial redistribution of the radiation dose is a technique also referred to as dose painting [44].

\section{Objective and outline of the thesis}

The aim of this thesis is to explore the use of functional imaging to characterize the tumour and tumour subvolumes, and subsequently to use these functional imaging characteristics for the prediction of patient prognosis, for patient stratification, and for treatment adaptation in non-small cell lung cancer and head and neck cancer. We hypothesize that functional imaging could substantially contribute to the optimization and personalization of radiotherapy.

Following the introduction, the thesis is divided in four parts. In Part 1, noninvasive imaging of drug uptake is described. The drug assessed in this part is the EGFR targeting monoclonal antibody cetuximab. The benefit of addition of cetuximab to radiotherapy widely varies between patients. Most likely, cetuximab treatment is beneficial in some, but not all, patients. Cetuximab requires adequate expression of EGFR in the tumour and cetuximab has to be able to reach the tumour. We hypothesize that the accessibility of the tumour to cetuximab might be a factor in treatment success and therefore an important predictive biomarker. To be able to visualize cetuximab uptake in the tumour, cetuximab is labelled with the PET tracer Zirconium-89. In Chapter 2, first the safety of ${ }^{89} \mathrm{Zr}$-cetuximab is evaluated in a clinical phase I trial. Next, the uptake of the labelled drug is quantified in a larger head and neck cohort, as described in Chapter 3.

Part 2 describes the use of the functional imaging techniques for visualizing hypoxia (HX4 PET) and vasculature using DCE-CT, for outcome prediction and treatment adaptation. In Chapter 4, the results of a clinical trial on nitroglycerin in NSCLC are reported. Nitroglycerin is a vasodilating drug, commonly used in the treatment of cardiac diseases, repurposed to reduce hypoxia levels in cancer. In a window-of-opportunity trial, hypoxia and tumour vasculature are assessed at baseline and after applying a nitroglycerin patch. The chapter reports the prognostic value of hypoxia PET and DCE-CT imaging at baseline. Changes between the baseline scans and scans with nitroglycerin patch are used to study the effect of nitroglycerin 
on tumour hypoxia and vasculature to predict the potential benefit of the addition of nitroglycerin to radiotherapy treatment. Chapter 5 explores hypoxia imaging for treatment adaptation. In this chapter, a planning study is presented assessing the feasibility of radiotherapy dose painting based on hypoxia HX4 PET imaging in NSCLC.

In Part 3, advanced image analysis techniques are used to combine multiple functional imaging parameters, i.e. multiparametric imaging, to assess multiple tumour characteristics simultaneously. In these two chapters, a data set of NSCLC patients containing anatomical CT, FDG PET, HX4 PET, and DCE-CT imaging is used. In Chapter 6, virtual hypoxia PET images are generated from FDG PET/CT and DCECT. Hypoxia imaging provides valuable information about treatment resistance and can be utilized for treatment adaptation (shown in Chapter 5). Hypoxia PET is unfortunately not widely available. We proposed a technique to extract information on hypoxia based on widely available imaging techniques (FDG PET/CT end DCECT). Chapter 7 introduces a methodology to combine hypoxia PET/CT, FDG PET/CT and DCE-CT on a subvolume level. The multiparametric characteristics of all patients are combined to identify high-risk subvolumes in NSCLC related to a worse patient prognosis.

To conclude, Chapter 8 contemplates the future steps to be taken in multiparametric imaging in oncology. Chapter 9 provides a general discussion and thoughts on future perspectives on functional imaging for personalized radiotherapy.

The outline of the thesis is schematically illustrated in Figure 1.3. 
Introduction
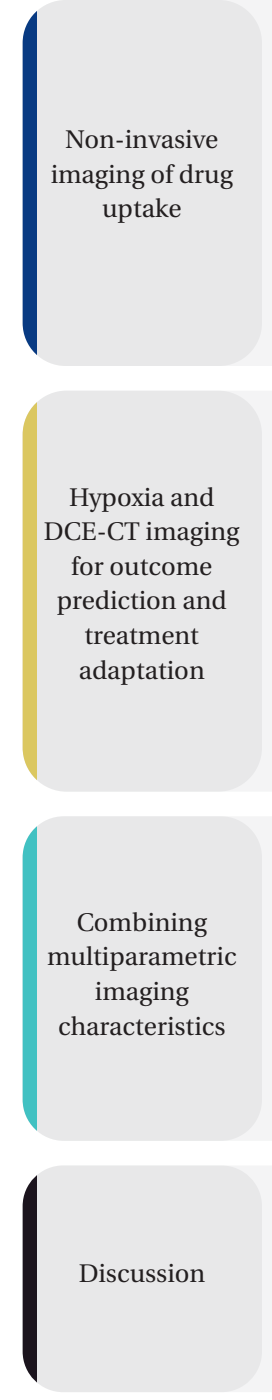

Main imaging

modality

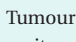

site

\section{Chapter 1}

Introduction and outline of the thesis

\section{Chapter 2}

PET imaging of zirconium-89 labelled cetuximab: a phase I trial in patients with head

${ }^{89} \mathrm{Zr}$-cetux PET

HNC and neck and lung cancer

\section{Chapter 3}

Quantitative assessment of Zirconium-89 labelled cetuximab using PET/CT imaging in patients with advanced head and neck cancer: a theragnostic approach

\section{Chapter 4}

Prognostic and predictive value of hypoxia HX4 PET and DCE-CT imaging in non-small cell lung cancer: results of a prospective window-of-opportunity clinical

\section{Chapter 5}

PET-based dose painting in non-small cell lung cancer: comparing uniform dose escalation with boosting hypoxic and metabolically active subvolumes

${ }^{89}$ Zr-cetux

HNC

HX4 PET/CT DCE-CT

NSCLC

HX4 PET/CT FDG PET/CT

NSCLC

\section{Chapter 6}

Predicting tumour hypoxia in non-small cell lung cancer by combining CT, FDG PET and dynamic contrast-enhanced CT

\section{Chapter 7}

Clustering of multiparametric functional imaging to identify high-risk subvolumes in non-small cell lung cancer

HX4 PET/CT FDG PET/CT DCE-CT

NSCLC

\section{Chapter 8}

The promise of multiparametric imaging in oncology: how do we move forward?

\section{Chapter 9}

General discussion and future perspectives

Figure 1.3. Schematic outline of the thesis. The main functional imaging modalities used are ${ }^{89} \mathrm{Zr}$-cetuximab PET/CT, FDG PET/CT, hypoxia HX4 PET/CT, and dynamic contrast-enhanced CT (DCE-CT). Patients with non-small cell lung cancer (NSCLC) and head and neck cancer (HNC) are examined. 


\section{References}

1. Breasted's translation of the Edwin Smith surgical papyrus. New England Journal of Medicine 206, 28-29 (1932).

2. Breasted, J. The Edwin Smith surgical papyrus (University of Chicago Press, 1930).

3. American Cancer Society. The history of cancer https://www.cancer.org/cancer/ cancer-basics/history-of-cancer/what-is-cancer.html.

4. Ferlay, J., Soerjomataram, I., Ervik, M., et al. GLOBOCAN 2012 v1.0, cancer incidence and mortality worldwide: IARC cancerbase no. $11 \mathrm{http}$ // globocan.iarc. fr.

5. Mayers, J. R. \& Vander Heiden, M. G. Nature and nurture: what determines tumor metabolic phenotypes? Cancer Res 77, 3131-4 (2017).

6. Lambert, A. W., Pattabiraman, D. R. \& Weinberg, R. A. Emerging biological principles of metastasis. Cell 168, 670-91 (2017).

7. Vogelstein, B., Papadopoulos, N., Velculescu, V. E., Zhou, S., Diaz L. A., J. \& Kinzler, K. W. Cancer genome landscapes. Science 339, 1546-58 (2013).

8. Hanahan, D. \& Weinberg, R. A. The hallmarks of cancer. Cell 100, 57-70 (2000).

9. Hanahan, D. \& Weinberg, R. A. Hallmarks of cancer: the next generation. Cell 144, 646-74 (2011).

10. Krause, D. S. \& Van Etten, R. A. Tyrosine kinases as targets for cancer therapy. $N$ Engl J Med 353, 172-87 (2005).

11. Sharma, S. V., Bell, D. W., Settleman, J. \& Haber, D. A. Epidermal growth factor receptor mutations in lung cancer. Nat Rev Cancer 7, 169-81 (2007).

12. Nicholson, R. I., Gee, J. M. W. \& Harper, M. E. EGFR and cancer prognosis. Eur J Cancer 37 Suppl 4, S9-15 (2001).

13. Potente, M., Gerhardt, H. \& Carmeliet, P. Basic and therapeutic aspects of angiogenesis. Cell 146, 873-87 (2011).

14. Goel, S., Duda, D. G., Xu, L., et al. Normalization of the vasculature for treatment of cancer and other diseases. Physiol Rev 91, 1071-121 (2011).

15. Jain, R. K. Normalization of tumor vasculature: an emerging concept in antiangiogenic therapy. Science 307, 58-62 (2005).

16. Gray, L. H., Conger, A. D., Ebert, M., Hornsey, S. \& Scott, O. C. A. The concentration of oxygen dissolved in tissues at the time of irradiation as a factor in radiotherapy. Br J Radiol 26, 638-48 (1953). 
17. Wilson, W. R. \& Hay, M. P. Targeting hypoxia in cancer therapy. Nat Rev Cancer 11, 393-410 (2011).

18. Warburg, O. On the origin of cancer cells. Science 123, 309-14 (1956).

19. Hirsch, F. R., Scagliotti, G. V., Mulshine, J. L., et al. Lung cancer: current therapies and new targeted treatments. Lancet 389, 299-311 (2017).

20. Herbst, R. S., Heymach, J. V. \& Lippman, S. M. Lung cancer. N Engl J Med 359, 1367-80 (2008).

21. Swanton, C. \& Govindan, R. Clinical implications of genomic discoveries in lung cancer. N Engl J Med 374, 1864-73 (2016).

22. Paidpally, V., Chirindel, A., Lam, S., Agrawal, N., Quon, H. \& Subramaniam, R. M. FDG-PET/CT imaging biomarkers in head and neck squamous cell carcinoma. Imaging Med 4, 633-47 (2012).

23. Argiris, A., Karamouzis, M. V., Raben, D. \& Ferris, R. L. Head and neck cancer. Lancet 371, 1695-709 (2008).

24. Barton, M. B., Jacob, S., Shafiq, J., et al. Estimating the demand for radiotherapy from the evidence: a review of changes from 2003 to 2012. Radiother Oncol 112, 140-4 (2014).

25. Vansteenkiste, J., Crinò, L., Dooms, C., et al. 2nd ESMO consensus conference on lung cancer: early-stage non-small-cell lung cancer consensus on diagnosis, treatment and follow-up. Ann Oncol 25, 1462-74 (2014).

26. Gregoire, V., Langendijk, J. A. \& Nuyts, S. Advances in radiotherapy for head and neck cancer. J Clin Oncol 33, 3277-84 (2015).

27. Van Dijk, L. K., Boerman, O. C., Kaanders, J. H. A. M. \& Bussink, J. PET imaging in head and neck cancer patients to monitor treatment response: a future role for EGFR-targeted imaging. Clin Cancer Res 21, 3602-9 (2015).

28. Fass, L. Imaging and cancer: a review. Mol Oncol 2, 115-52 (2008).

29. Baumann, M., Krause, M., Overgaard, J., et al. Radiation oncology in the era of precision medicine. Nat Rev Cancer 16, 234-49 (2016).

30. Alonzi, R. \& Hoskin, P. Functional imaging in clinical oncology: magnetic resonance imaging- and computerised tomography-based techniques. Clin Oncol (R Coll Radiol) 18, 555-70 (2006).

31. Ter-Pogossian, M. M., Phelps, M. E., Hoffman, E. J. \& Mullani, N. A. A positronemission transaxial tomograph for nuclear imaging (PETT). Radiology 114, 8998 (1975). 
32. Kelloff, G. J., Hoffman, J. M., Johnson, B., et al. Progress and promise of FDG-PET imaging for cancer patient management and oncologic drug development. Clin Cancer Res 11, 2785-808 (2005).

33. Chapman, J. D., Franko, A. J. \& Sharplin, J. A marker for hypoxic cells in tumours with potential clinical applicability. Br J Cancer 43, 546-50 (1981).

34. Peeters, S. G. J. A., Zegers, C. M. L., Lieuwes, N. G., et al. A comparative study of the hypoxia PET tracers $\left[{ }^{18} \mathrm{~F}\right]-\mathrm{HX} 4,\left[{ }^{18} \mathrm{~F}\right]-\mathrm{FAZA}$, and $\left[{ }^{18} \mathrm{~F}\right]$-FMISO in a preclinical tumor model. Int J Radiat Oncol Biol Phys 91, 351-9 (2015).

35. Van Dongen, G. A. M. S., Visser, G. W. M., Lub-de Hooge, M. N., de Vries, E. G. \& Perk, L. R. Immuno-PET: a navigator in monoclonal antibody development and applications. Oncologist 12, 1379-89 (2007).

36. Wright, B. D. \& Lapi, S. E. Designing the magic bullet? The advancement of immuno-PET into clinical use. J Nucl Med 54, 1171-4 (2013).

37. Axel, L. Cerebral blood flow determination by rapid-sequence computed tomography: theoretical analysis. Radiology 137, 679-86 (1980).

38. Miles, K. A. Perfusion CT for the assessment of tumour vascularity: which protocol? Br J Radiol 76 Spec No 1, S36-42 (2003).

39. Klotz, E., Haberland, U., Glatting, G., et al. Technical prerequisites and imaging protocols for CT perfusion imaging in oncology. Eur J Radiol 84, 2359-67 (2015).

40. Troost, E. G. C., Thorwarth, D. \& Oyen, W. J. G. Imaging-based treatment adaptation in radiation oncology. J Nucl Med 56, 1922-9 (2015).

41. O’Connor, J. P. B., Aboagye, E. O., Adams, J. E., et al. Imaging biomarker roadmap for cancer studies. Nat Rev Clin Oncol 14, 169-86 (2017).

42. Gregoire, V., Haustermans, K., Geets, X., Roels, S. \& Lonneux, M. PET-based treatment planning in radiotherapy: a new standard? J Nucl Med 48 Suppl 1, 68S$77 \mathrm{~S}$ (2007).

43. Jeraj, R., Bradshaw, T. \& Simoncic, U. Molecular imaging to plan radiotherapy and evaluate its efficacy. J Nucl Med 56, 1752-65 (2015).

44. Ling, C. C., Humm, J., Larson, S., et al. Towards multidimensional radiotherapy (MD-CRT): biological imaging and biological conformality. Int J Radiat Oncol Biol Phys 47, 551-60 (2000). 



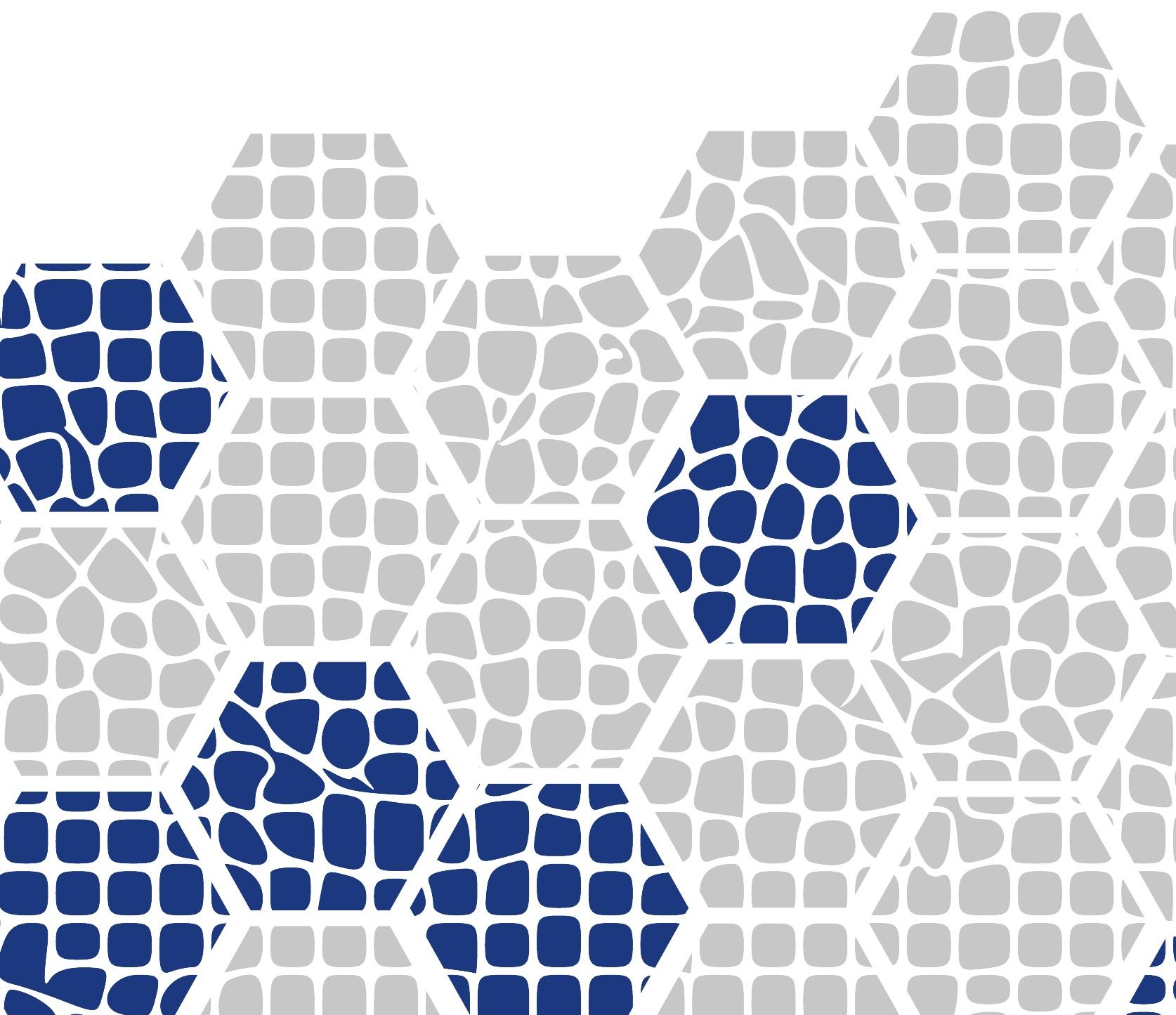


Non-invasive imaging of drug uptake

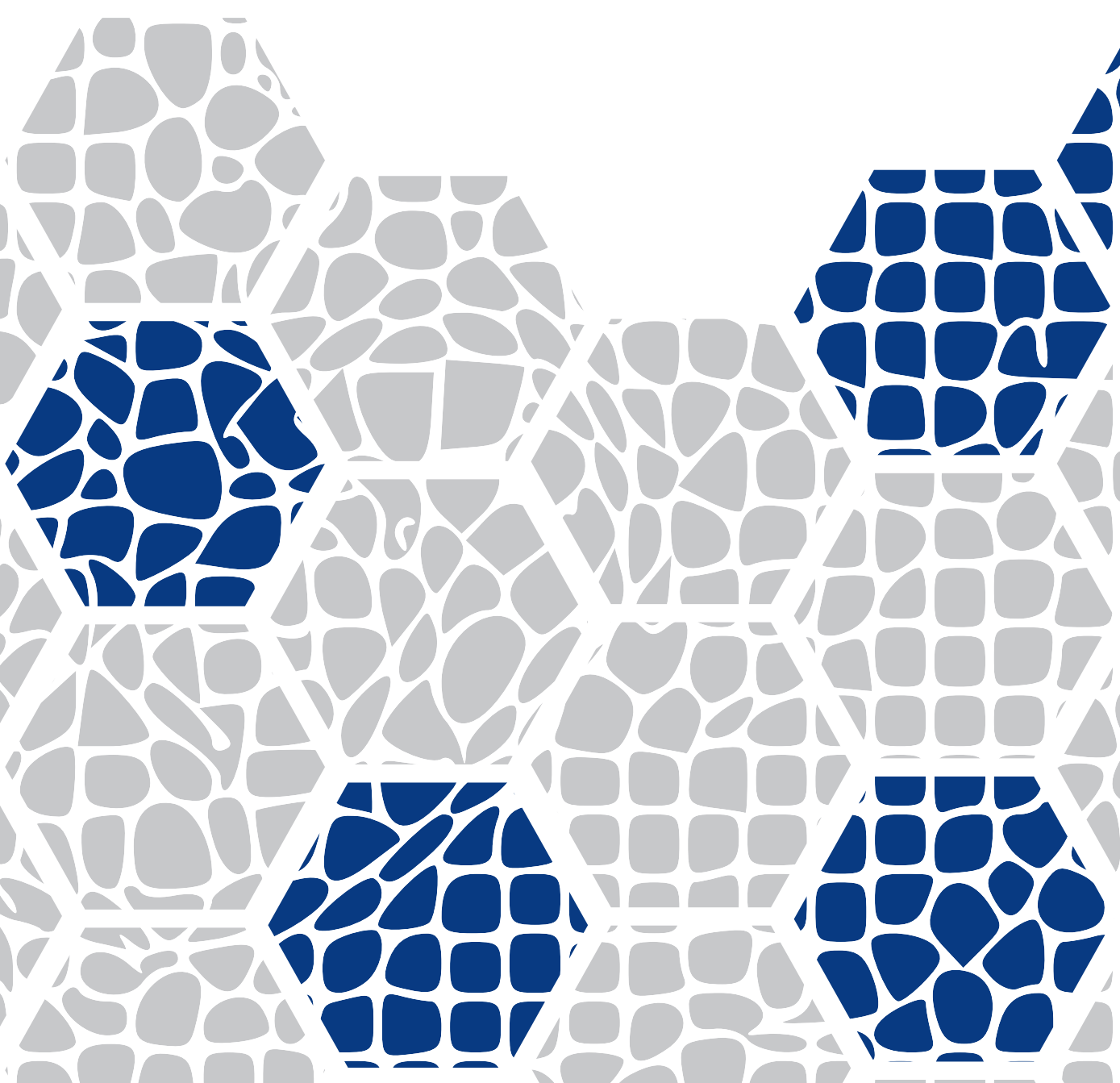




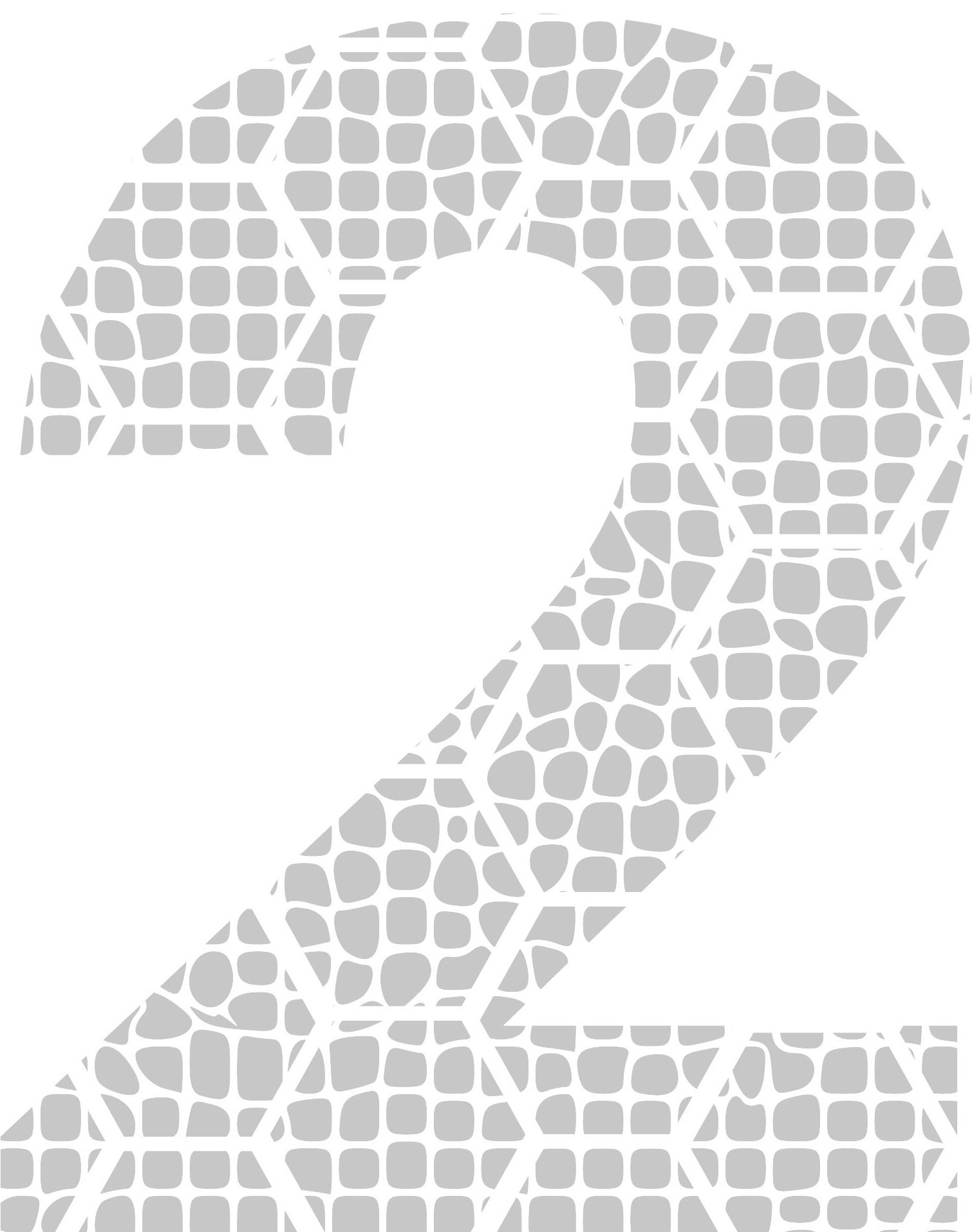




\section{PET imaging of Zirconium-89 labelled cetuximab: a phase I trial in patients with head and neck and lung cancer}

\section{Judith van Loon*, Aniek J.G. Even*, Hugo J.W.L. Aerts, Michel Öllers,}

Frank Hoebers, Wouter van Elmpt, Ludwig Dubois, Anne-Marie C. Dingemans, Roy I. Lalisang, Pascal Kempers, Boudewijn Brans, Véronique J.L. Winnepenninckx, Ernst-Jan Speel, Erik Thunnissen, Kim M. Smits, Ronald Boellaard, Daniëlle J. Vugts, Dirk De Ruysscher, Philippe Lambin 


\begin{abstract}
Background and purpose PET imaging of cetuximab uptake may help select cancer patients with the highest chance of benefiting from cetuximab treatment. The aim of this phase I trial was to determine the safety of the PET tracer ${ }^{89} \mathrm{Zr}$-cetuximab and to assess the PET uptake in the tumour.

Methods Two dose schedules were used; two consecutive doses of $60 \mathrm{MBq}{ }^{89} \mathrm{Zr}$-cetuximab or a single dose of $120 \mathrm{MBq}$, both preceded by $400 \mathrm{mg} / \mathrm{m}^{2}$ of unlabelled cetuximab. Toxicity (CTCAE 3.0) was scored twice weekly. PET/CT scans were acquired on day 4, 5, and 6 (step 1) or 5, 6, and 7 (step 2). Because tumour uptake could not be assessed satisfactorily, a third step was added including EGFR overexpressing tumours.
\end{abstract}

Results Nine patients were included (six NSCLC; three HNC). No additional toxicity was associated with administration of ${ }^{89} \mathrm{Zr}$-cetuximab compared to standard cetuximab. A tumour-to-background ratio (TBR) larger than 1 was observed in all but one patient, with a maximum of 4.6. TBR was not different between dose schedules. There was a trend for higher TBRs at intervals larger than five days after injection of ${ }^{89} \mathrm{Zr}$ cetuximab.

Conclusions Both presented ${ }^{89} \mathrm{Zr}$-cetuximab administration schedules are safe. The recommended dose for future trials is $60 \mathrm{MBq}$, with a minimum time interval for scanning of six days. 


\section{Introduction}

Monoclonal antibodies are increasingly used in anticancer treatment to specifically target receptors at the surface of tumour cells, either as a monotherapy or in combination with radiotherapy or chemotherapy. Cetuximab is such a monoclonal antibody that specifically blocks the epidermal growth factor receptor (EGFR) which is overexpressed in many human malignancies [1,2]. EGFR activation and overexpression appear to be important tumour cell mechanisms in the development of resistance to radiation and chemotherapy, resulting in decreased rates of local tumour control and survival [3].

A randomized phase III trial in head and neck cancer showed improved survival by adding cetuximab to radiotherapy [4]. However, cetuximab combined with radiotherapy failed to show any benefit over chemoradiotherapy [5]. The addition of cetuximab to chemoradiotherapy did also not show benefit in head and neck cancer [6] or non-small cell lung cancer [7, 8]. Selection of patients with the highest chance of benefit from cetuximab treatment is of obvious relevance, also in view of its high costs. The mechanisms underlying clinical response or resistance to EGFR targeting cetuximab treatment combined with radiotherapy are, however, largely unknown $[9,10]$.

Non-invasive visualization and quantification of tumour uptake of cetuximab may contribute significantly to the selection of patients and determination of the needed dosage [11]. Several methods using nuclear imaging have been proposed [12, 13].

As the biologic half-life of cetuximab in blood is 65-95 hours [14], a radioactive tracer with a long half-life is needed to visualize its uptake. Zirconium-89 $\left({ }^{89} \mathrm{Zr}\right)$, with a half-life of approximately 78 hours, is an example of such a positron emission tomography (PET) tracer that can successfully be labelled to cetuximab and has shown promising results in animal models [15-17]. In a preclinical study, uptake of ${ }^{89} \mathrm{Zr}$ cetuximab was demonstrated only in EGFR positive tumours. However, it was shown that ${ }^{89} \mathrm{Zr}$-cetuximab uptake did not correlate with EGFR expression levels, implying that pharmacokinetic and -dynamic factors influence the cetuximab accumulation in tumours [16].

Here, we report the results of a phase I study with as primary aim determining the safety of Zirconium-89 labelled cetuximab. Quantification of ${ }^{89} \mathrm{Zr}$-cetuximab uptake in the tumour is a secondary end point. In addition, we aimed to get an indication of the optimal radioactivity dose and imaging time point to direct future phase II studies. 


\section{Methods}

\section{Patient selection}

Patients with a histologically confirmed solid cancer without curative treatment options were eligible. Inclusion criteria were: WHO performance status zero to two; adequate bone marrow, adequate hepatic and renal function; life expectancy of more than three months and written informed consent. Patients with a recent ( $<$ three months) myocardial infarction, uncontrolled infectious disease, pregnancy, previous administration of cetuximab or concurrent treatment with anticancer agents or radiotherapy were excluded.

\section{Study design}

A study design with two different dose schedules was used (Figure 2.1). In both steps, ${ }^{89} \mathrm{Zr}$-cetuximab was administered within one hour after administration of the loading dose of unlabelled cetuximab. Six patients were included, three in each dose schedule. If in any patient grade two or higher toxicity was observed related to ${ }^{89} \mathrm{Zr}$ cetuximab administration, thee extra patients would be included in this dose step. When at maximum one out of six patients experienced grade two toxicity, the step was considered safe.

The study design anticipated the future aim, which is to determine the tumour uptake of ${ }^{89} \mathrm{Zr}$-cetuximab before and during therapy. Therefore, in the first dose schedule (step 1), toxicity of two consecutive low doses of ${ }^{89} \mathrm{Zr}$-cetuximab was investigated. A standard loading dose of $400 \mathrm{mg} / \mathrm{m}^{2}$ cetuximab, followed by $10 \mathrm{mg}$ of ${ }^{89} \mathrm{Zr}$-cetuximab (60 MBq) was administered on day 0 . A second injection with a maintenance dose of $250 \mathrm{mg} / \mathrm{m}^{2}$ of cetuximab, followed by $10 \mathrm{mg}$ of ${ }^{89} \mathrm{Zr}$-cetuximab (60 MBq) was administered on day 14 .

As a larger radioactivity dose of ${ }^{89} \mathrm{Zr}$-cetuximab is possibly needed to obtain the best image quality, toxicity of a single larger dose was investigated in a second dose schedule (step 2). A loading dose of $400 \mathrm{mg} / \mathrm{m}^{2}$ of cetuximab was administered followed by injection of $10 \mathrm{mg}$ of cetuximab labelled with $120 \mathrm{MBq}^{89} \mathrm{Zr}$.

During inclusion in step 2, a study amendment was written based on the first results of the image analysis. It was decided that if the secondary endpoint (assessment of tumour ${ }^{89} \mathrm{Zr}$-cetuximab uptake) could not be satisfactorily assessed based on the first six patients, a third step would be added in which three patients were included with an EGFR overexpressing tumour based on recent immunohistochemistry. For these patients, the administration schedule was the same as in step 2. The study protocol was approved by the Medical Ethics Review Committee and the radiation safety committee. The study is listed in ClinicalTrials.gov number NCT00691548. 


\section{Step 1}

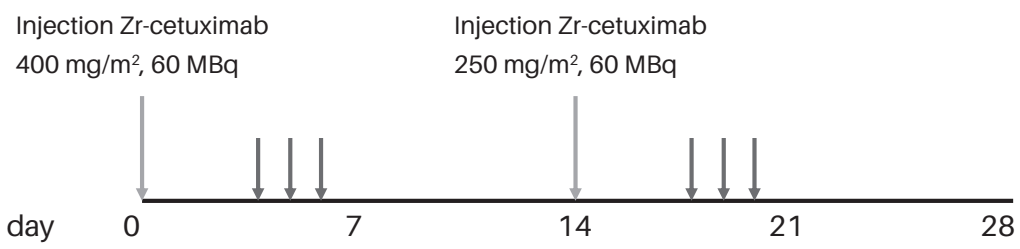

Step 2

Injection Zr-cetuximab

$400 \mathrm{mg} / \mathrm{m}^{2}, 120 \mathrm{MBq}$

day

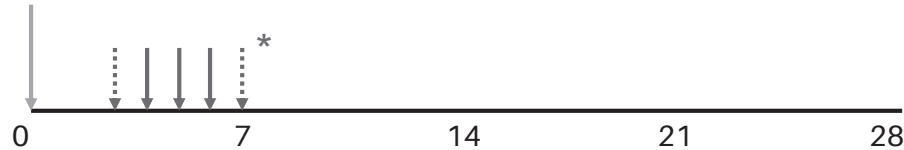

* days of scanning in step 2 depend on imaging results in step 1

Figure 2.1. Timeline of the study: ${ }^{89} \mathrm{Zr}$-cetuximab injection (grey arrows) and the acquiring of ${ }^{89} \mathrm{Zr}$-cetuximab PET/CT scans (black arrows). In step 2, PET/CT scans on day 3 and 7 after injection were optional and depended on the imaging results in step 1. If in step 1, two or more patients had a higher tumour-to-background ratio (TBR) on day 4 than on day 5 , an additional scan on day 3 would be performed. If in step 1, two or more patients had a higher TBR on day 6 than on day 5 , an additional scan on day 7 would be performed. Patients included in step 3 were scanned at one time point only, based on the imaging results of step 1 and 2.

\section{EGFR expression and mutation status}

In all patients, biopsies of the primary tumour were taken before the start of treatment. For patients included in step 3, a recent biopsy of the primary tumour or from a metastatic lesion had to be available, without any antitumour treatment between the biopsy and inclusion. After inclusion, EGFR expression and mutation status (exons 18-21) as well as KRAS mutation (codons 12 and 13) were assessed on these biopsies [18]. EGFR expression was analysed with the EGFR pharmDx qualitative immunohistochemical kit system (Novocastra and Dako, Denmark). To quantify EGFR expression, both EGFR membrane staining intensity and the percentage of EGFR expressing cells were taken into account. EGFR membrane staining intensity was expressed as a score between 0 (none) and 3+ (strong). The percentage of cells staining at different intensities was assessed visually. Subsequently, EGFR immunohistochemistry (IHC) scores were defined on a scale of 0-300 using a formula combining percentage of staining cells and staining intensity [19]. Tumours were divided in showing low $(<200)$ and high $(\geq 200)$ EGFR expression. This threshold was based on the results of the FLEX study [20, 21]. 


\section{Toxicity scoring}

Toxicity was assessed during treatment, and twice weekly after treatment until 14 days after the last injection, according to the CTCAE 3.0 scoring system. At baseline, and on day 7 and day 14 after injection, blood testing was performed for haematology, kidney and liver function.

The most common side effect of cetuximab administration that was anticipated for was skin toxicity (acneform rash) [22]. As skin toxicity is a known side effect of unlabelled cetuximab administration and this trial aimed to investigate the safety of labelled cetuximab, skin toxicity was not regarded as a dose limiting side effect.

\section{Synthesis of ${ }^{89} \mathrm{Zr}$-cetuximab}

${ }^{89} \mathrm{Zr}$ was produced by a (p,n) reaction on natural ${ }^{89} \mathrm{Y}$ as described by Verel et al. [23]. Subsequently, labelling of ${ }^{89} \mathrm{Zr}$ to the mAb cetuximab was performed as previously reported [24].

\section{PET/CT imaging}

PET/CT imaging was performed at day 4, 5, and 6 after injection in dose step 1 . Imaging intervals in step 2 were adapted based on the TBRs at subsequent days in step 1. Patients included in step 3 were scanned at one time point only, based on the imaging results of step 1 and 2 . Furthermore, an optional ${ }^{18} \mathrm{~F}$ fluorodeoxyglucose (FDG) PET scan was performed within the study period, at least 24 hours before or 12 days after ${ }^{89} \mathrm{Zr}$-cetuximab administration ( $\geq$ four times the half-life of cetuximab). In the first patient that underwent an FDG PET scan after the ${ }^{89} \mathrm{Zr}$-cetuximab administration, an extra PET/CT scan was performed at day 12, preceding FDG injection, to assess remaining activity associated with the ${ }^{89} \mathrm{Zr}$ cetuximab administration.

\section{Image analysis}

Tumour sites and normal tissues were manually delineated on one of the ${ }^{89} \mathrm{Zr}$ cetuximab PET/CT scans (day 6 or 7) by the same observer (JvL). For one patient, delineations were performed on the FDG PET/CT scan. Tumour sites were delineated based on the CT using FDG PET information when available. To quantify uptake in muscle and liver, a transversal CT slice of the subscapular muscle and the liver was delineated. The resulting regions of interest were subsequently projected onto the other ${ }^{89} \mathrm{Zr}$-cetuximab PET/CT scans and FDG PET scan through coregistration of the corresponding $\mathrm{CT}$ images using rigid registration. 
The mean, maximum and peak standardized uptake value $\left(\mathrm{SUV}_{\text {mean }}, \mathrm{SUV}_{\max }\right.$ and $\mathrm{SUV}_{\text {peak }}$, respectively) were determined using in-house developed dedicated software. The tumour SUV $V_{\text {peak }}$ was defined by calculating the mean SUV in a sphere with a diameter of $1.2 \mathrm{~cm}$ within the tumour region with the highest activity. Tumour-tobackground ratio (TBR) was calculated by dividing tumour $S U V_{\text {peak }}$ by $S U V_{\text {mean }}$ in the aortic arch.

For patients in whom an additional ${ }^{18} \mathrm{~F}$-FDG PET scan was performed, the FDG PET and ${ }^{89} \mathrm{Zr}$-cetuximab PET uptake were visually compared.

\section{Results}

Patient characteristics are shown in Supplementary Table S2.1, tumour characteristics in Table 2.1. Nine patients, with a median age of 62 years (range: 53-75), were included. The third patient was excluded from the study before injection of ${ }^{89} \mathrm{Zr}$ labelled cetuximab, because of malignant hypertension during administration of the unlabelled cetuximab. The blood pressure normalized within two hours of observation without further consequences. Therefore an extra patient (patient 4) was included in dose step 1. Only two patients were included in step 3 of the study. Given the slow accrual, and since this step was not necessary to reach the primary endpoint, it was decided to close this step prematurely.

From the nine patients included, six had non-small cell lung cancer (NSCLC) and three had head and neck cancer (HNC). All patients had previously undergone anticancer therapy. In one patient, EGFR expression and mutation, and KRAS mutation status of the primary tumour could not be assessed due to insufficient quantity of histological material. In the other patients, six of the seven primary tumours showed high EGFR expression, while none showed a mutation of the EGFR gene. An example of IHC staining in a biopsy with high EGFR expression is shown in Supplementary Figure 2.2 (patient 6). In the only patient with low EGFR expression, the primary tumour showed a mutation in the KRAS gene. One of the two patients included in step 3 showed high EGFR expression on a recent tumour biopsy. The recent specimen of the second patient contained insufficient material to allow a reliable EGFR analysis.

Each patient received ${ }^{89} \mathrm{Zr}$-cetuximab administration as planned. Patients included in step 1 and 2 underwent the ${ }^{89} \mathrm{Zr}$ PET/CT scans at three consecutive days, except for patient 6 who could not undergo the first scan due to pain. Patient 4 , included in step 1, was scanned at day 5-7 instead of day 4-6 because of logistical reasons. In two patients (patient 2 and 4) enrolled in the first dose step, the series of PET/CT scans after the second injection could not be performed due to pain and dyspnoea. Patients included in step 3 were scanned at day 6 after injection. Four 
Table 2.1. Tumour characteristics, and FDG PET and ${ }^{89} \mathrm{Zr}$-cetuximab PET uptake in the tumour and normal tissues.

\begin{tabular}{|c|c|c|c|c|c|c|c|c|c|c|c|c|c|c|c|}
\hline \multirow[b]{3}{*}{ Patient } & \multicolumn{5}{|c|}{ Primary tumour } & \multirow[t]{3}{*}{ Schedule } & FDG PET & \multicolumn{8}{|c|}{${ }^{89} \mathrm{Zr}$-cetuximab PET } \\
\hline & \multirow[b]{2}{*}{ Site } & \multirow[b]{2}{*}{ Histology } & \multicolumn{2}{|c|}{ Mutation $^{* *}$} & \multirow[b]{2}{*}{ EGFR IHC score } & & \multicolumn{5}{|c|}{ Tumour } & \multicolumn{2}{|c|}{ Liver } & \multicolumn{2}{|c|}{ Muscle } \\
\hline & & & KRAS & EGFR & & & $\mathrm{SUV}_{\max }$ & $\mathrm{TBR}_{\text {peak }}$ & $\mathrm{SUV}_{\text {peak }}$ & $\mathrm{SUV}_{\max }$ & $\mathrm{SUV}_{\text {mean }}$ & SUV $_{\max }$ & $\mathrm{SUV}_{\text {mean }}$ & $\mathrm{SUV}_{\max }$ & $\mathrm{SUV}_{\text {mean }}$ \\
\hline 1 & Lung & SCC & NA & NA & NA & step 1 & 11.3 & 2.8 & 4.7 & 7.1 & 1.8 & NA & NA & 3.4 & 0.6 \\
\hline 2 & Lung & LCC & + & - & 80 & step 1 & NA & 3.1 & 4.3 & 7.8 & 2.1 & NA & NA & 2.6 & 0.6 \\
\hline $3^{*}$ & Lung & LCC & NA & NA & NA & step 1 & NA & NA & NA & NA & NA & NA & NA & NA & NA \\
\hline 4 & Lung & AC & - & - & 280 & step 1 & NA & 4.6 & 3.8 & 7.6 & 1.3 & 8.1 & 5.5 & 1.4 & 0.4 \\
\hline 5 & Lung & SCC & - & - & 240 & step 2 & 10.2 & 1.0 & 1.2 & 1.6 & 0.8 & 7.8 & 5.7 & 1.0 & 0.2 \\
\hline 6 & Lung & $\mathrm{AC}$ & - & - & 290 & step 2 & 7.6 & 1.7 & 3.6 & 6.6 & 1.9 & 9.9 & 9.2 & 1.3 & 0.3 \\
\hline 7 & Oropharynx & SCC & - & - & 300 & step 2 & 11.3 & 3.2 & 3.0 & 4.3 & 2.0 & 7.0 & 5.3 & 1.6 & 0.3 \\
\hline 8 & Oropharynx & MC & - & - & 270 & step 3 & NA & 1.4 & 4.4 & 6.2 & 3.2 & 7.1 & 6.8 & 1.8 & 0.8 \\
\hline 9 & Oropharynx & SCC & - & - & 290 & step 3 & NA & 1.5 & 4.8 & 6.9 & 3.0 & 18.4 & 12.5 & 1.2 & 0.4 \\
\hline
\end{tabular}

*Excluded patient.

${ }^{* *}$ KRAS: codons 12 and 13; EGFR: exons 18-21.

Abbreviations: SCC = squamous cell carcinoma; LCC = large cell carcinoma; $\mathrm{AC}=$ adenocarcinoma; $\mathrm{MC}=$ muco-epidermoid carcinoma; $\mathrm{NA}=$ not assessable . 
patients underwent an FDG PET scan within the study period, at an interval of 12 or 13 days after the injection of ${ }^{89} \mathrm{Zr}$-cetuximab.

An overview of the toxicity per patient is presented in Supplementary Table 2.2. No toxicity other than skin rash was observed in any of the patients. Of the eight patients that received the full cetuximab administration, three patients experienced grade one toxicity, and four experienced grade two acne form rash. No changes were observed for haematological, kidney and liver function compared to baseline.

${ }^{89} \mathrm{Zr}$-cetuximab PET images of all patients are shown in Figure 2.2 (coronal) and Figure 2.3 (transversal). Tumour and normal tissue uptake values of ${ }^{89} \mathrm{Zr}$-cetuximab are shown in Table 2.1. All but one patient had a peak TBR larger than 1 at any of the imaging time points. For each patient, the ${ }^{89} \mathrm{Zr}$-cetuximab image with the highest TBR was selected for further analyses. For patients in dose step 1, only the scans after the first ${ }^{89} \mathrm{Zr}$-cetuximab injection were analysed, since for two of the three patients PET/CT scans could not be acquired in the second week. The average peak TBR was 2.4 (range: $1.0-4.6$ ). The average tumour $\mathrm{SUV}_{\max }$ and $\mathrm{SUV}_{\text {mean }}$ were 6.0 (range: 1.6-7.8) and 2.0 (range: $0.8-3.2$ ), respectively. The average $\mathrm{SUV}_{\text {max }}$ and $\mathrm{SUV}_{\text {mean }}$ for the liver were 9.7 (range: 7.0-18.4) and 7.5 (range: 5.3-12.5). For muscle, the average $\mathrm{SUV}_{\max }$ and $\mathrm{SUV}_{\text {mean }}$ were 1.8 (range: 1.0-3.4) and 0.4 (range: 0.2-0.8). Both $\mathrm{SUV}_{\max }$ and $\mathrm{SUV}_{\text {mean }}$ of the tumour were significantly higher than the muscle $\mathrm{SUV}_{\max }$ and $\mathrm{SUV}_{\text {mean }}$.

The FDG PET and ${ }^{89} \mathrm{Zr}$-cetuximab scans of the four patients with an FDG PET/CT scan are shown in Figure 2.4. Visual comparison showed a remarkable mismatch between FDG PET and ${ }^{89} \mathrm{Zr}$-cetuximab PET uptake in one patient (patient 1, Figure 2.4). No direct relationship was observed between the EGFR IHC score and TBR.

For the patients in dose step 1, the average peak TBR at day 4, 5, and 6 after the first injection was 1.3 (range: 1.2-1.4), 2.1 (range: 1.7-2.7) and 3.1 (range: 1.7-4.6), respectively (Figure 2.5). As the highest TBR was found at day 6 after injection, imaging in dose step 2 was performed at day 5,6 , and 7 . For patients in dose step 2, the average peak TBR at day 5, 6, and 7 after injection was 1.7 (range: 0.9-2.4), 1.6 (range: 1.0-2.7) and 2.0 (range: 0.9-3.2) (Figure 2.5). The peak TBR of patients included in step 3 was 1.4 and 1.5 (day 6). At the regular imaging time points, the highest peak TBR was seen in patient 4 (4.6), at day 6 after injection. In patient 1, an extra PET/CT scan was performed at day 12 after injection. The TBR at this time point was higher than the maximum TBR for this patient at the regular imaging time points (2.8 at day 12 versus 1.7 at day 6$)$. 


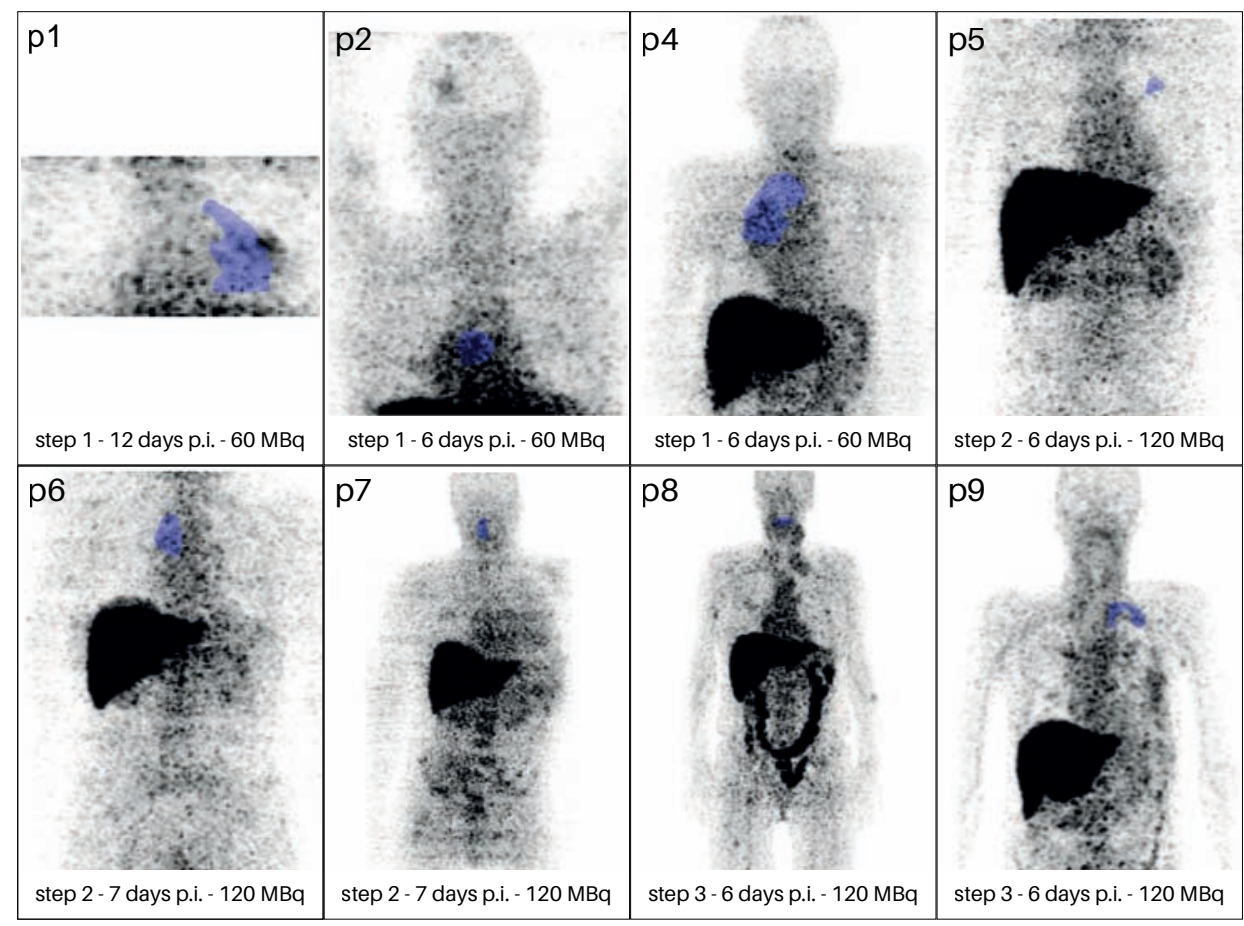

Figure 2.2. ${ }^{89} \mathrm{Zr}$-cetuximab PET maximum intensity projections of all patients. The GTV of the primary tumour is overlaid in blue. The notes underneath the images indicate in which dose step the patients were included, how many days postinjection the displayed images were acquired and the dose of ${ }^{89} \mathrm{Zr}$-cetuximab $(2 \times 60 \mathrm{MBq}$ or $1 \times 120 \mathrm{MBq})$ administered. The scans with the highest TBR in the primary tumour were selected. For dose step 1 , the images after the first injection were analysed.

\section{Discussion}

The current phase I trial is the first study in HNC and NSCLC evaluating the safety of ${ }^{89} \mathrm{Zr}$-cetuximab. It is a first step towards a new patient selection method for cetuximab treatment or the addition of cetuximab to radiotherapy. ${ }^{89} \mathrm{Zr}$-cetuximab has until now only been evaluated in human patients with colorectal cancer [25]. In this study, no additional toxicity was associated with ${ }^{89} \mathrm{Zr}$-cetuximab administration. Acne form eruption $\leq$ grade two was observed in $88 \%$ of patients, a rate comparable to that found with therapeutic administration of cetuximab $[4,22,26]$. One patient developed malignant hypertension during the administration of unlabelled cetuximab. Although rare, this side effect has been described previously [27]. 


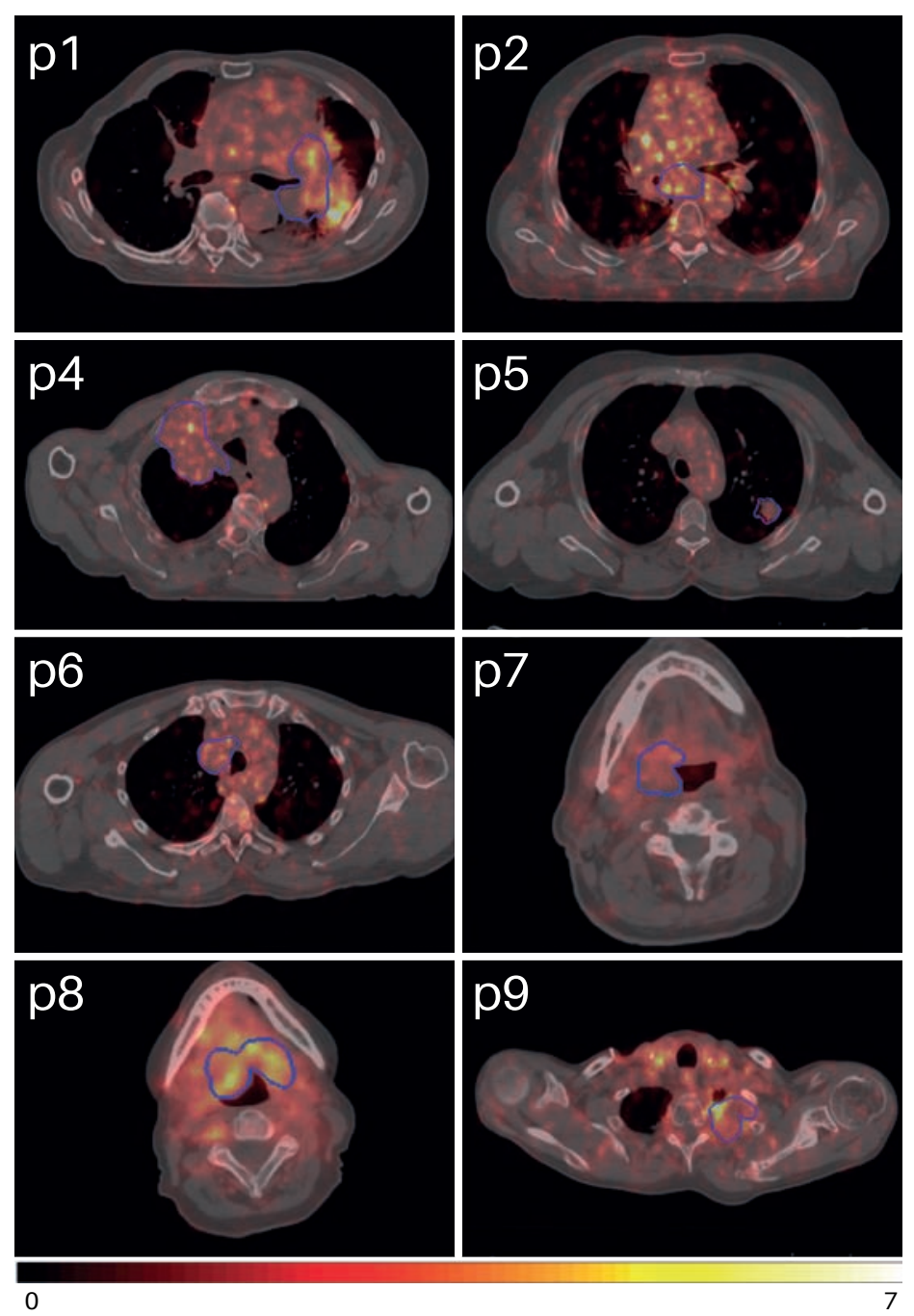

Figure 2.3. ${ }^{89} \mathrm{Zr}$-cetuximab PET/CT images at tumour level showing uptake in SUV. The primary tumour is delineated in blue. The scans with the highest TBR in the primary tumour were selected. 

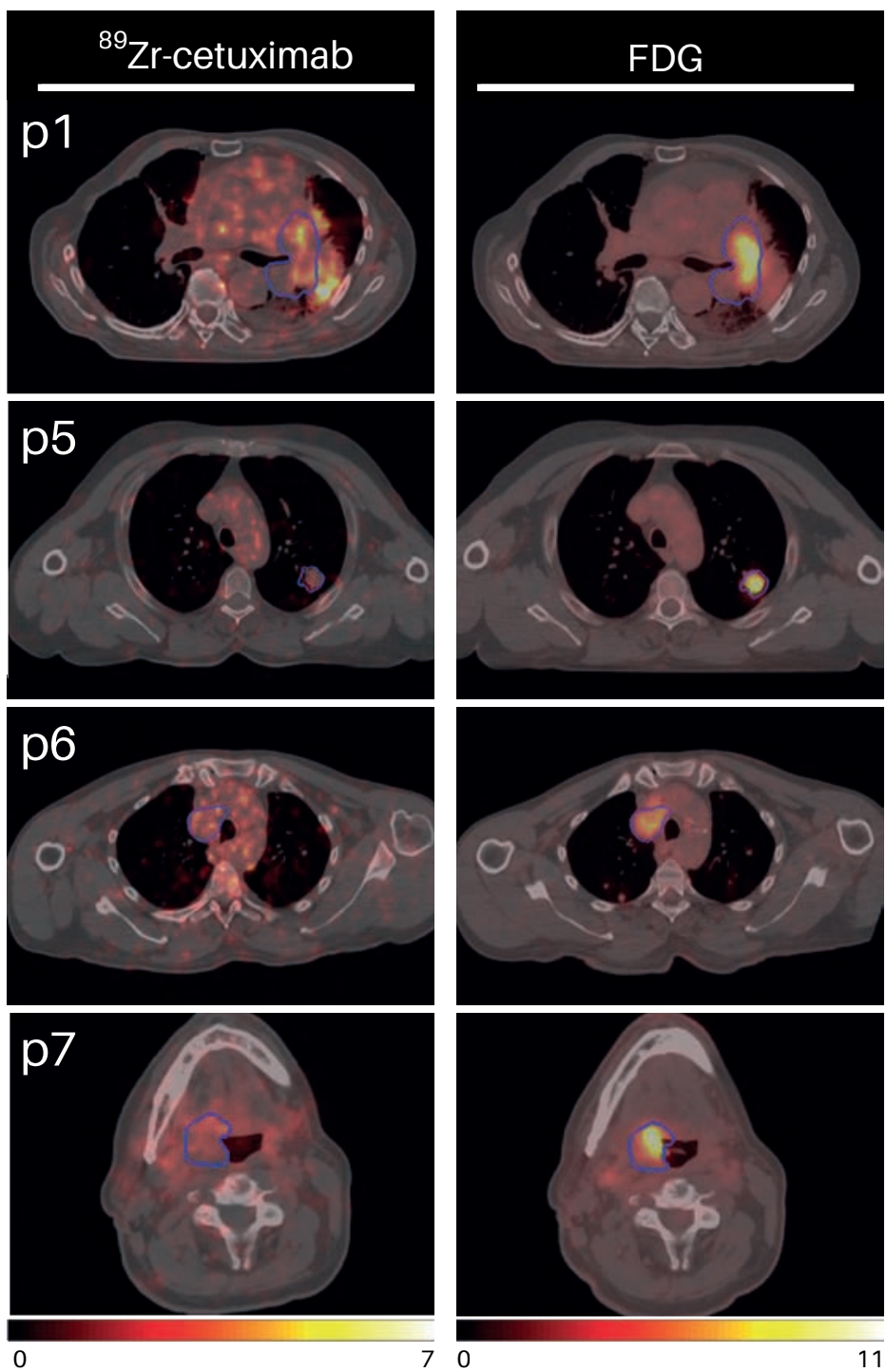

Figure 2.4. Correlation between ${ }^{89} \mathrm{Zr}$-cetuximab PET and FDG PET uptake for the four patients receiving both scans. PET uptake is shown in SUV. The primary tumour is delineated in blue. The ${ }^{89} \mathrm{Zr}$-cetuximab scans with the highest TBR in the primary tumour were selected. 


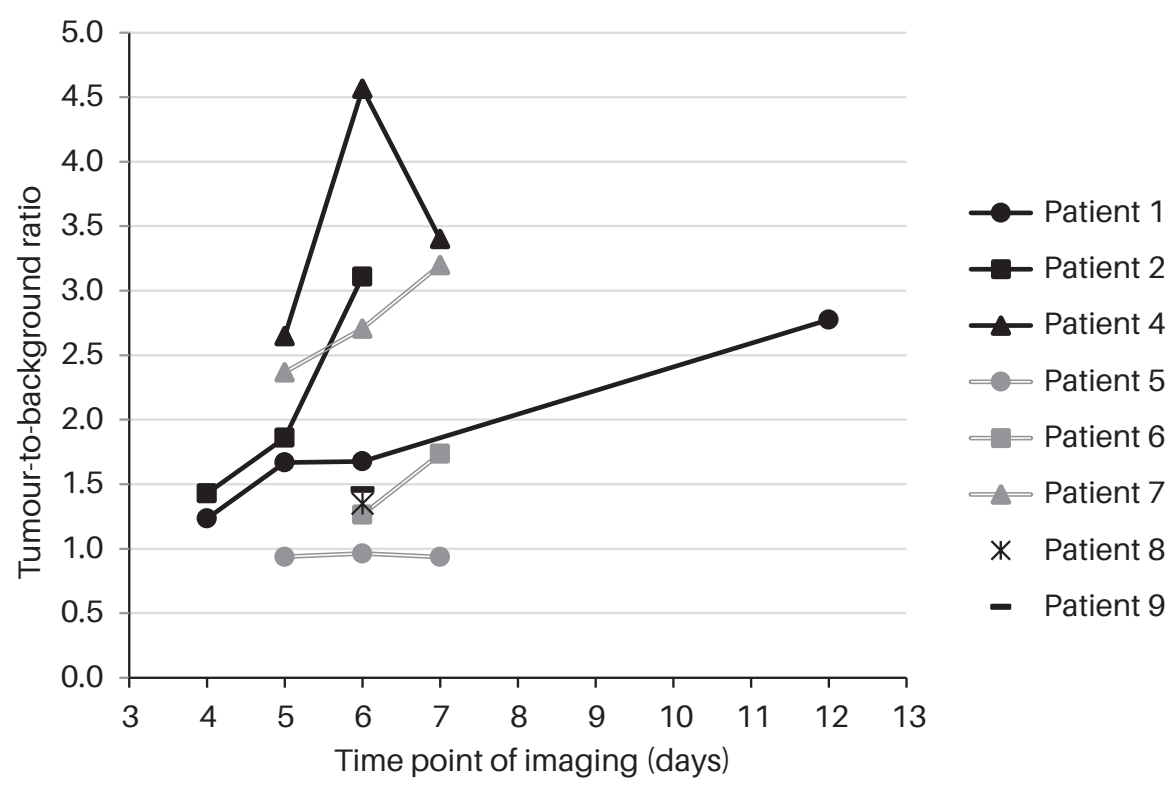

Figure 2.5. ${ }^{89} \mathrm{Zr}$-cetuximab tumour-to-background ratios at different time points after injection of the tracer. The patients displayed with a black line were included in dose step 1 ; the patients with a grey line in dose step 2; the patients with a single black marker in dose step 3.

\section{Tumour uptake of ${ }^{89} \mathrm{Zr}$-cetuximab}

It should be emphasized that no definitive conclusions can be drawn given the small amount of patients as well as the heterogeneity in tumour type and previous treatment. All but one patient that completed the study showed a peak TBR larger than 1. Visually, however, the ${ }^{89} \mathrm{Zr}$-cetuximab images showed a rather patchy distribution, without evident specific uptake of ${ }^{89} \mathrm{Zr}$-cetuximab within the tumour. Visually comparing the ${ }^{89} \mathrm{Zr}$-cetuximab images of NSCLC and HNC patients showed more discernible tumour uptake be present in at least one of the HNC patients (patient 8, Figure 2.3).

No direct relationship was observed between EGFR IHC score and TBR, similar to the findings in our preclinical study [16]. There are several considerations that argue a direct relationship between EGFR expression assessed in biopsy specimens and response to cetuximab treatment. Firstly, as expression of EGFR can be induced by radiation and chemotherapy, it is possible that an initially EGFR negative tumour may become positive early during therapy, thereby still benefiting from cetuximab treatment $[28,29]$. Secondly, EGFR expressing tumours will not respond to cetuximab when cetuximab does not reach therapeutic concentrations in the tumour because of e.g. interstitial pressure and vascular perfusion changes [30]. 


\section{Recommended ${ }^{89} \mathrm{Zr}$-cetuximab dose and time interval for scanning}

A large divergence was observed in the time curves of the TBR. Hence, no optimal imaging time point can be derived from these data. However, we advise a time delay of at least six days after injection, as five of the six patients that were scanned at day 5 and 6 showed a higher TBR at day 6 . This is in line with a study in colorectal cancer that also found 6 days postinjection to be the optimal imaging time point [25]. In the only patient that was scanned after a substantially longer time interval (12 days after administration), the highest TBR was found at this time point. This late rise in TBR suggests that a later imaging time point might result in more optimal tumour visualization. Due to decay, however, accurate quantification at such long time intervals may be biased by increased noise levels.

Although no definitive conclusions can be drawn regarding the dosage of labelled cetuximab in view of the different tumour characteristics in the two dose schedules, the current results do not indicate that a higher dose would result in more optimal TBRs. Therefore, taking into account the ALARA (as low as reasonably achievable) principle, a dose of $60 \mathrm{MBq}$ of ${ }^{89} \mathrm{Zr}$-cetuximab is recommended for future study.

\section{Recommendations for further study}

There are some other factors of which the influence on tumour visualization should be assessed in future studies. First, the loading dose administered in this study ( $400 \mathrm{mg} / \mathrm{m}^{2}$ ), which is the standard therapeutic regimen, might be suboptimal to enable adequate tumour visualization. A loading dose of cetuximab is required as cetuximab first binds irreversibly to EGFR expressing liver cells. Therefore tumour cells are only targeted after the liver has been saturated. This rationale is supported by animal studies. In addition, the positive correlation between skin toxicity and response to cetuximab implies that skin saturation only occurs after the liver has been saturated [31, 32]. The liver $\mathrm{SUV}_{\max }$ and $\mathrm{SUV}_{\text {mean }}$ in the current trial were rather high, implying that EGF receptors in the liver were still not saturated by the loading dose.

Based on the promising clinical results of adding cetuximab to radiotherapy in HNC [4], and the imaging data from the current study suggesting specific ${ }^{89} \mathrm{Zr}$ cetuximab PET uptake in at least one of the HNC patients, a clinical trial was started (ARTFORCE head and neck trial, ClinicalTrials.gov: NCT01504815 [33]). Unfortunately, due to slow accrual, complexity of ${ }^{89} \mathrm{Zr}$-cetuximab imaging for both patients and radiotherapy departments, and discontinuation of the funding of cetuximab, the study was amended and ${ }^{89} \mathrm{Zr}$-cetuximab imaging, and cetuximab treatment were discontinued.

In conclusion, the administration of ${ }^{89} \mathrm{Zr}$-cetuximab to image in vivo cetuximab uptake is safe and not associated with any additional toxicity compared to unlabelled 
cetuximab. The recommended time interval between cetuximab administration and imaging is at least six days. The recommended ${ }^{89} \mathrm{Zr}$-cetuximab dose is $60 \mathrm{MBq}$. Further research is needed to explore the optimal loading dose of non-labelled cetuximab and the relationship between ${ }^{89} \mathrm{Zr}$-cetuximab and EGFR expression.

\section{Acknowledgements}

We acknowledge financial support from the CTMM framework (AIRFORCE), EU 6th and 7th framework program (Euroxy, Metoxia and ARTFORCE), Interreg (www.eurocat.info), Kankeronderzoekfonds Limburg (Health Foundation Limburg), the National Institute of Health (NIH-USA U01 CA 143062-01) and the Dutch Cancer Society (KWF UM 2008-4210, 2009-4454, 2011-5020 and KWF MAC 2013-6425). 


\section{References}

1. Rowinsky, E. K. The erbB family: targets for therapeutic development against cancer and therapeutic strategies using monoclonal antibodies and tyrosine kinase inhibitors. Annu Rev Med 55, 433-57 (2004).

2. Herbst, R. S. Review of epidermal growth factor receptor biology. Int J Radiat Oncol 59, 21-6 (2004).

3. Ang, K. K., Berkey, B. A., Tu, X., et al. Impact of epidermal growth factor receptor expression on survival and pattern of relapse in patients with advanced head and neck carcinoma. Cancer Res 62, 7350-6 (2002).

4. Bonner, J. A., Harari, P. M., Giralt, J., et al. Radiotherapy plus cetuximab for squamous-cell carcinoma of the head and neck. N Engl J Med 354, 567-78 (2006).

5. Petrelli, F., Coinu, A., Riboldi, V., et al. Concomitant platinum-based chemotherapy or cetuximab with radiotherapy for locally advanced head and neck cancer: a systematic review and meta-analysis of published studies. Oral Oncol 50, 1041-8 (2014).

6. Ang, K. K., Zhang, Q., Rosenthal, D. I., et al. Randomized phase III trial of concurrent accelerated radiation plus cisplatin with or without cetuximab for stage III to IV head and neck carcinoma: RTOG 0522. J Clin Oncol 32, 2940-50 (2014).

7. Bradley, J. D., Masters, G., Hu, C., et al. An intergroup randomized phase III comparison of standard-dose (60 Gy) versus high-dose (74 Gy) chemoradiotherapy (CRT) + / - cetuximab (cetux) for stage III non-small cell lung cancer (NSCLC): results on cetux from RTOG 0617. J Thorac Oncol 8, S2-S1410 (2013).

8. Walraven, I., van den Heuvel, M., van Diessen, J., et al. Long-term follow-up of patients with locally advanced non-small cell lung cancer receiving concurrent hypofractionated chemoradiotherapy with or without cetuximab. Radiother Oncol 118, 442-6 (2016).

9. Kriegs, M., Gurtner, K., Can, Y., et al. Radiosensitization of NSCLC cells by EGFR inhibition is the result of an enhanced p53-dependent G1 arrest. Radiother Oncol 115, 120-7 (2015).

10. Stegeman, H., Kaanders, J. H., van der Kogel, A. J., et al. Predictive value of hypoxia, proliferation and tyrosine kinase receptors for EGFR-inhibition and radiotherapy sensitivity in head and neck cancer models. Radiother Oncol 106, 383-9 (2013). 
11. Van Dongen, G. A. M. S., Visser, G. W. M., Lub-de Hooge, M. N., de Vries, E. G. \& Perk, L. R. Immuno-PET: a navigator in monoclonal antibody development and applications. Oncologist 12, 1379-89 (2007).

12. Koi, L., Bergmann, R., Brüchner, K., et al. Radiolabeled anti-EGFR-antibody improves local tumor control after external beam radiotherapy and offers theragnostic potential. Radiother Oncol 110, 362-9 (2014).

13. Van Dijk, L. K., Hoeben, B. A. W., Stegeman, H., et al. ${ }^{111}$ In-cetuximab-F(ab') 2 SPECT imaging for quantification of accessible epidermal growth factor receptors (EGFR) in HNSCC xenografts. Radiother Oncol 108, 484-8 (2013).

14. Fracasso, P. M., Burris III, H., Arquette, M. A., et al. A phase 1 escalating single-dose and weekly fixed-dose study of cetuximab: pharmacokinetic and pharmacodynamic rationale for dosing. Clin Cancer Res 13, 986-93 (2007).

15. Vosjan, M. J. W. D., Perk, L. R., Visser, G. W. M., et al. Conjugation and radiolabeling of monoclonal antibodies with zirconium-89 for PET imaging using the bifunctional chelate p-isothiocyanatobenzyl-desferrioxamine. Nat Protoc 5, 739-43 (2010).

16. Aerts, H. J. W. L., Dubois, L., Perk, L., et al. Disparity between in vivo EGFR expression and ${ }^{89} \mathrm{Zr}$-labeled cetuximab uptake assessed with PET. J Nucl Med 50, 123-31 (2009).

17. Aerts, H. J. W. L., Dubois, L., Hackeng, T. M., et al. Development and evaluation of a cetuximab-based imaging probe to target EGFR and EGFRvIII. Radiother Oncol 83, 326-32 (2007).

18. Heinemann, V., Stintzing, S., Kirchner, T., Boeck, S. \& Jung, A. Clinical relevance of EGFR- and KRAS-status in colorectal cancer patients treated with monoclonal antibodies directed against the EGFR. Cancer Treat Rev 35, 262-71 (2009).

19. Lee, H. J., Xu, X., Choe, G., et al. Protein overexpression and gene amplification of epidermal growth factor receptor in nonsmall cell lung carcinomas: comparison of four commercially available antibodies by immunohistochemistry and fluorescence in situ hybridization study. Lung Cancer 68, 375-82 (2010).

20. Rüschoff, J., Kerr, K. M., Grote, H. J., et al. Reproducibility of immunohistochemical scoring for epidermal growth factor receptor expression in non-small cell lung cancer: round robin test. Arch Pathol Lab Med 137, 1255-61 (2013).

21. Pirker, R., Pereira, J. R., von Pawel, J., et al. EGFR expression as a predictor of survival for first-line chemotherapy plus cetuximab in patients with advanced non-small-cell lung cancer: analysis of data from the phase 3 FLEX study. Lancet Oncol 13, 33-42 (2012). 
22. Galimont-Collen, A. F. S., Vos, L. E., Lavrijsen, A. P. M., Ouwerkerk, J. \& Gelderblom, H. Classification and management of skin, hair, nail and mucosal side-effects of epidermal growth factor receptor (EGFR) inhibitors. Eur J Cancer 43, 845-51 (2007).

23. Verel, I., Visser, G. W. M., Boellaard, R., Stigter-van Walsum, M., Snow, G. B. \& van Dongen, G. A. M. S. ${ }^{89} \mathrm{Zr}$ immuno-PET: comprehensive procedures for the production of ${ }^{89}$ Zr-labeled monoclonal antibodies. J Nucl Med 44, 1271-81 (2003).

24. Perk, L. R., Visser, G. W. M., Vosjan, M. J. W. D., et al. ${ }^{89} \mathrm{Zr}$ as a PET surrogate radioisotope for scouting biodistribution of the therapeutic radiometals ${ }^{90} \mathrm{Y}$ and ${ }^{177} \mathrm{Lu}$ in tumor-bearing nude mice after coupling to the internalizing antibody cetuximab. J Nucl Med 46, 1898-906 (2005).

25. Menke-van der Houven van Oordt, C. W., Gootjes, E. C., Huisman, M. C., et al. ${ }^{89} \mathrm{Zr}$-cetuximab PET imaging in patients with advanced colorectal cancer. Oncotarget 6, 30384-93 (2015).

26. Gatzemeier, U., von Pawel, J., Vynnychenko, I., et al. First-cycle rash and survival in patients with advanced non-small-cell lung cancer receiving cetuximab in combination with first-line chemotherapy: a subgroup analysis of data from the FLEX phase 3 study. Lancet Oncol 12, 30-7 (2011).

27. Iakovlev, V. V., Pintilie, M., Morrison, A., Fyles, A. W., Hill, R. P. \& Hedley, D. W. Effect of distributional heterogeneity on the analysis of tumor hypoxia based on carbonic anhydrase IX. Lab Invest 87, 1206-17 (2007).

28. Contessa, J. N., Reardon, D. B., Todd, D., et al. The inducible expression of dominant-negative epidermal growth factor receptor-CD533 results in radiosensitization of human mammary carcinoma cells. Clin Cancer Res 5, 40511 (1999).

29. Schmidt-Ullrich, R. K., Contessa, J. N., Dent, P., et al. Molecular mechanisms of radiation-induced accelerated repopulation. Radiat Oncol Investig 7, 321-30 (1999).

30. De Bree, R., Kuik, D. J., Quak, J. J., et al. The impact of tumour volume and other characteristics on uptake of radiolabelled monoclonal antibodies in tumour tissue of head and neck cancer patients. Eur J Nucl Med 25, 1562-5 (1998).

31. Burtness, B., Goldwasser, M. A., Flood, W., Mattar, B. \& Forastiere, A. A. Phase III randomized trial of cisplatin plus placebo compared with cisplatin plus cetuximab in metastatic/recurrent head and neck cancer: an Eastern Cooperative Oncology Group study. J Clin Oncol 23, 8646-54 (2005). 
32. Tolmachev, V., Rosik, D., Wållberg, H., et al. Imaging of EGFR expression in murine xenografts using site-specifically labelled anti-EGFR ${ }^{111}$ In-DOTAZEGFR:2377 Affibody molecule: aspect of the injected tracer amount. Eur J Nucl Med Mol Imaging 37, 613-22 (2010).

33. Heukelom, J., Hamming, O., Bartelink, H., et al. Adaptive and innovative Radiation Treatment FOR improving Cancer treatment outcomE (ARTFORCE); a randomized controlled phase II trial for individualized treatment of head and neck cancer. BMC Cancer 13, 84 (2013). 


\section{Supplementary data}

Table S2.1. Patient characteristics.

\begin{tabular}{lrlrrrl}
\hline Patient & Age (years) & Sex & Length $(\mathrm{m})$ & Weight $(\mathrm{kg})$ & WHO PS & Prior therapy \\
\hline 1 & 72 & Male & 1.85 & 75 & 0 & CTx \\
2 & 61 & Male & 1.76 & 82 & 1 & CTx, RT \\
$3^{*}$ & 55 & Female & 1.56 & 61 & 0 & CTx \\
4 & 75 & Male & 1.79 & 74 & 2 & CTx \\
5 & 53 & Male & 1.80 & 91 & 1 & CTx \\
6 & 63 & Male & 1.78 & 86 & 0 & CTx \\
7 & 63 & Male & 1.86 & 95 & 1 & RT, CTx \\
8 & 59 & Female & 1.67 & 60 & 1 & S, CTx \\
9 & 62 & Male & 1.71 & 60 & 1 & CTx, RT \\
\hline
\end{tabular}

${ }^{*}$ Excluded patient.

Abbreviations: WHO PS = World Health Organisation performance status; CTx = chemotherapy; $\mathrm{RT}$ = radiotherapy; $\mathrm{S}$ = surgery.

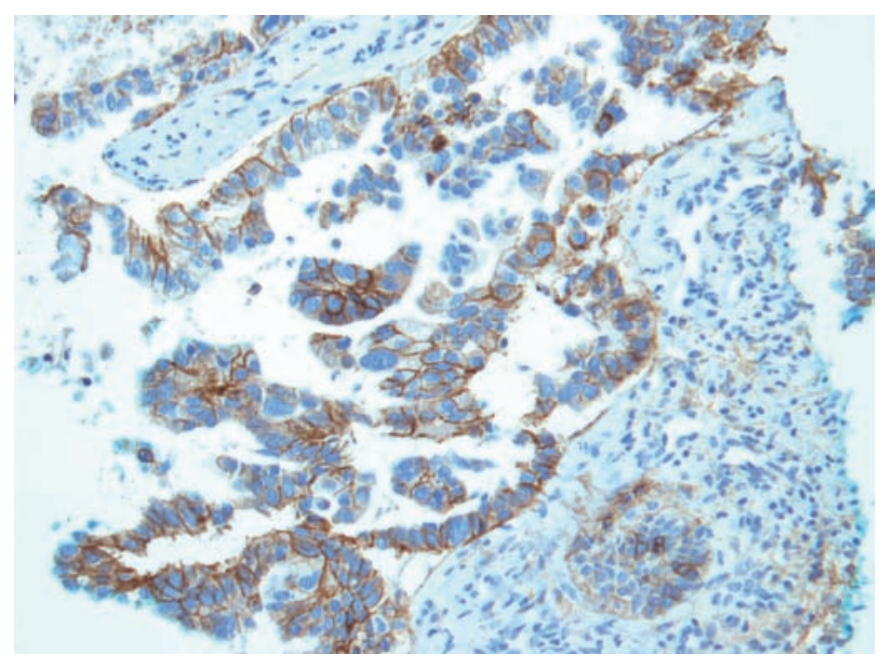

Figure S2.1. EGFR immunostaining. 
Table 2.2. Toxicity scoring.

\begin{tabular}{|c|c|c|c|c|c|c|c|c|}
\hline \multirow[t]{2}{*}{ Patient } & \multirow[t]{2}{*}{ Injected activity (MBq) } & \multirow[t]{2}{*}{ Vital signs $^{* *}$} & \multicolumn{5}{|c|}{ Toxicity scoring $^{* *}$} & \multirow[t]{2}{*}{ Medication $^{* *}$} \\
\hline & & & Haematology & Liver function & Renal function & Skin & Symptoms & \\
\hline 1 & $2 \times 60$ & 0 & 0 & 0 & 0 & 2 & 0 & $1^{* * *}$ \\
\hline 2 & $2 \times 60$ & 0 & 0 & 0 & 0 & 1 & 0 & 0 \\
\hline $3^{*}$ & none & NA & NA & NA & NA & NA & NA & NA \\
\hline 4 & $2 \times 60$ & 0 & 0 & 0 & 0 & 0 & 0 & 0 \\
\hline 5 & 120 & 0 & 0 & 0 & 0 & 2 & 0 & $1^{* * *}$ \\
\hline 6 & 120 & 0 & 0 & 0 & 0 & 2 & 0 & $1^{* * *}$ \\
\hline 7 & 120 & 0 & 0 & 0 & 0 & 1 & 0 & 0 \\
\hline 8 & 120 & 0 & 0 & 0 & 0 & 2 & 0 & 0 \\
\hline 9 & 120 & 0 & 0 & 0 & 0 & 1 & 0 & $1^{* * *}$ \\
\hline
\end{tabular}

Toxicity was scored before study entry (baseline) and at day 7 and day 14 after cetuximab administration.

$\mathrm{NA}=$ not assessed because of premature exclusion from the study.

*Excluded patient.

${ }^{* *}$ Every change in vital signs or medication compared to the baseline values was scored, with "0" indicating no change.

${ }^{* * *}$ Doxycycline 100 mg, Hydroxyzine 25 mg, Metronidazolcream 10 mg/g. 


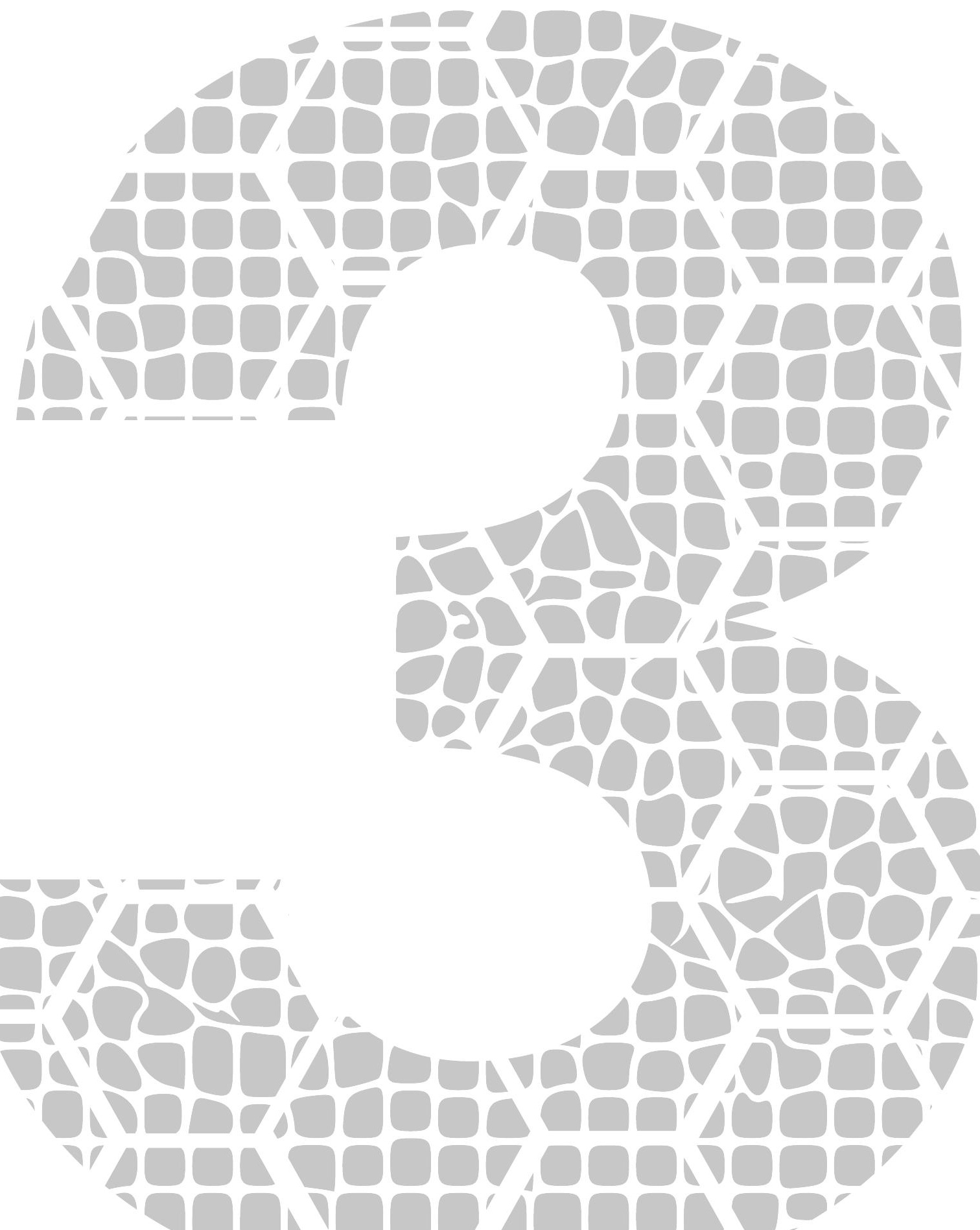




\section{Quantitative assessment of Zirconium-89 labelled cetuximab using PET/CT imaging in patients with advanced head and neck cancer: a theragnostic approach}

Aniek J.G. Even*, Olga Hamming-Vrieze ${ }^{*}$, Wouter van Elmpt, Véronique J.L. Winnepenninckx, Jolien Heukelom, Margot E.T. Tesselaar, Wouter V. Vogel, Ann Hoeben, Catharina M.L. Zegers, Daniëlle J. Vugts, Guus A.M.S. van Dongen, Harry Bartelink, Felix M. Mottaghy, Frank Hoebers, 


\begin{abstract}
Introduction Biomarkers predicting treatment response to the monoclonal antibody cetuximab in locally advanced head and neck squamous cell carcinomas (LAHNSCC) are lacking. We hypothesize that tumour accessibility is an important factor in treatment success of the EGFR targeting drug. We quantified uptake of cetuximab labelled with Zirconium-89 $\left({ }^{89} \mathrm{Zr}\right)$ using PET/CT imaging.

Material and Methods Seventeen patients with stage III-IV LAHNSCC received a loading dose unlabelled cetuximab, followed by $10 \mathrm{mg}$ $54.5 \mathrm{MBq} \pm 9.6 \mathrm{MBq}{ }^{89} \mathrm{Zr}$-cetuximab. PET/CT images were acquired at either 3 and 6 , or 4 and 7 days postinjection. ${ }^{89} \mathrm{Zr}$-cetuximab uptake was quantified using standardized uptake value (SUV) and tumour-tobackground ratio (TBR), and correlated to EGFR immunohistochemistry. TBR was compared between scan days to determine optimal timing.
\end{abstract}

Results Uptake of ${ }^{89} \mathrm{Zr}$-cetuximab varied between patients (day 6-7: $S_{\text {peak }}$ range 2.5-6.2). The TBR increased significantly $(49 \% \pm 28 \%$, $\mathrm{p}<0.01)$ between the first $(1.1 \pm 0.3)$ and second scan $(1.7 \pm 0.6)$. Between groups with a low and high EGFR expression a significant difference in $\mathrm{SUV}_{\text {mean }}$ (2.1 versus 3.0) and $\mathrm{SUV}_{\text {peak }}$ (3.2 versus 4.7) was found, however, not in TBR. Data is available at www.cancerdata.org (DOI: 10.17195/candat.2016.11.1).

Conclusion In conclusion, ${ }^{89} \mathrm{Zr}$-cetuximab PET imaging shows a large interpatient variety in LAHNSCC and provides additional information over FDG PET and EGFR expression. Validation of the predictive value is recommended with scans acquired at 6 or 7 days postinjection. 


\section{Introduction}

Locally-advanced head and neck squamous cell carcinomas (LAHNSCC) are challenging to treat. The majority of patients presents with locally advanced cancers at the time of diagnosis [1]. Although advances in surgery, radiotherapy and systemic therapy have improved survival over the last decade, the prognosis remains poor [2]. Patients with advanced locoregional disease require multimodality treatment [3]. For (functionally) irresectable tumours, radiotherapy is combined with concurrent cisplatin [4, 5], or with the targeted drug cetuximab [6]. Cetuximab is a human-mouse chimeric monoclonal antibody targeting the epidermal growth factor receptor (EGFR). This receptor activates several pathways that are involved in cell proliferation and survival. The EGF receptor is overexpressed in most LAHNSCC and is related to radio- and chemotherapy resistance $[7,8]$. Cetuximab binds to the extracellular domain of EGFR, blocks ligand binding and, as a result, prevents receptor activation [9-12].

Radiotherapy combined with either cisplatin or cetuximab have both shown improved treatment results over radiotherapy alone $[6,13]$. However, addition of cetuximab to chemoradiotherapy, or substituting radiotherapy combined cisplatin by cetuximab did not show any additional benefit [14-16]. Most likely not all patients will benefit equally from the same treatment, for example due to intertumour heterogeneity or patient related factors, making patient tailored treatment essential. Several measures were proposed for predicting cetuximab treatment efficacy, including drug-induced skin rash, EGFR protein expression and EGFR gene mutations $[17,18]$. So far, the predictive value of these markers has been inconclusive. We hypothesize that the accessibility of the cetuximab into the tumour is an important predictive marker in the treatment efficacy [19]. In tumours lacking EGFR expression, response to the targeted drug is unexpected regardless of accessibility, while in tumours with an EGFR overexpression, the accessibility of the tumour is expected to be a determining factor in drug uptake. Imaging with radioactive labelled cetuximab could be used to non-invasively quantify the uptake of cetuximab. Ultimately, drug uptake imaging could be applied in the clinic for pretreatment patient selection (e.g. in combination with decision support systems [20, 21]), and treatment evaluation during therapy.

Since antibodies such as cetuximab have a long half-life in the blood pool (69-95 hours) radioactive labelling with the long-lived positron emitter Zirconium-89 $\left({ }^{89} \mathrm{Zr}\right)$ was chosen (half-life of 78 hours) $[22,23]$. Aerts et al. [19] proved in an animal study that in vivo imaging of ${ }^{89} \mathrm{Zr}$-cetuximab is feasible and also showed a disparity between ${ }^{89} \mathrm{Zr}$-cetuximab uptake and EGFR expression of the tumour cells. Moreover, it was shown in a phase I first in human study that ${ }^{89} \mathrm{Zr}$-cetuximab can be safely administered to patients [24]. 
The main aims of this study were to quantify the uptake of ${ }^{89} \mathrm{Zr}$-cetuximab in the tumour and involved lymph nodes in patients with LAHNSCC, and to determine optimal timing of imaging after ${ }^{89} \mathrm{Zr}$-cetuximab administration. The secondary aim was to correlate ${ }^{89} \mathrm{Zr}$-cetuximab uptake with EGFR expression, and metabolic activity as determined by ${ }^{18} \mathrm{~F}$-fluorodeoxyglucose (FDG) PET/CT scan.

\section{Materials and methods}

\section{Patient selection and treatment protocol}

Patients with previously untreated, histologically proven stage III-IV, T3-T4 squamous cell carcinoma of the oropharynx, oral cavity or hypopharynx, were included in the multicentre clinical ARTFORCE trial (ClinicalTrials.gov: NCT01504815). The trial was approved by the appropriate Medical Ethics Review Committee. After giving written informed consent, patients underwent a double randomization: 1. standard radiation dose of $70 \mathrm{~Gy}$, or dose redistribution to the primary tumour to a maximum of $84 \mathrm{~Gy}$ on the FDG-avid area and a dose gradient from 70 to $64 \mathrm{~Gy}$ in the remainder of the primary tumour; 2 . cisplatin or cetuximab concurrently with radiotherapy. The study protocol is described in detail by Heukelom et al. [25]. For all treatment arms, pretreatment imaging consisted of one FDG PET/CT scan and two ${ }^{89} \mathrm{Zr}$-cetuximab PET/CT scans. The ${ }^{89} \mathrm{Zr}$-cetuximab PET/CT scans were solely used for research purposes and did not influence any clinical decisions.

\section{${ }^{18}$ F-FDG PET/CT image acquisition}

Pretreatment FDG PET/CT scans were acquired at least one day before ${ }^{89} \mathrm{Zr}$-cetuximab administration using the standard clinical protocol following EANM guidelines [26]. All patients were scanned in treatment position: on a flat table top and immobilized using a personalized radiotherapy mask with neck rest and with their arms by their sides.

\section{${ }^{89} \mathrm{Zr}$-cetuximab PET/CT image acquisition and analysis}

Labelling of cetuximab with Zirconium-89 was performed as described by Verel et al. [27]. Data on the quality of the labelling process can be found in the Supplementary data. Patients first received an intravenous loading dose of unlabelled cetuximab of $400 \mathrm{mg} / \mathrm{m}^{2}$, directly followed by $10 \mathrm{mg}{ }^{89}$ Zirconium labelled cetuximab of $55 \mathrm{MBq}$ (range: $29-62 \mathrm{MBq}) .{ }^{89} \mathrm{Zr}$-cetuximab PET/CT images were acquired at 4 and 7 days postinjection (p.i.), corresponding to day -3 and day 1 of radiotherapy. Alternatively, patients could be scanned on day 3 and 6 p.i. for logistic reasons. If 
${ }^{89} \mathrm{Zr}$-cetuximab PET/CT imaging and the first radiotherapy fraction were scheduled on the same day, the PET scan was always acquired before radiotherapy. Patients were scanned in radiotherapy treatment position wearing a personalized radiotherapy mask on either a Philips Gemini TF 16 PET/CT scanner (Philips Healthcare, Best, the Netherlands) or Siemens Biograph TruePoint scanner (Siemens Medical Solutions, Erlangen, Germany). Scans were acquired with a minimum time per bed position of 3 minutes. The Philips PET images were reconstructed using an ordered-subsets time of flight reconstruction technique (BLOB-OS-TF), with 3 iterations and 33 subsets. The Siemens images were reconstructed with a point spread function algorithm (PSF), with either 4 iterations and 14 subsets or 3 iterations and 21 subsets. One scan was reconstructed using the 2D OSEM algorithm with 4 iterations and 8 subsets. All scans were corrected for attenuation, scatter, and ${ }^{89} \mathrm{Zr}$ decay. Images acquired with the Philips Gemini PET/CT system were additionally smoothed with a Gaussian filter (full width at half maximum of $7 \mathrm{~mm}$ ) to match the noise levels of the different scanners, as described by Makris et al. [28]. The PET/CT images are publicly available at www.cancerdata.org [29].

\section{Tumour delineation}

Gross tumour volumes of the primary tumour $\left(\mathrm{GTV}_{\text {prim }}\right)$ and involved lymph nodes $\left(G V_{\ln }\right)$ were delineated by an experienced radiation oncologist during the clinical radiation treatment planning process and subsequently propagated to the different scans for further analysis. The delineations were performed either on a dedicated planning CT scan, or on the pretreatment FDG PET/CT scan. In case a dedicated planning CT was acquired, the CT scan was first rigidly registered to the CT scan of the FDG PET/CT scan after which the contours were propagated to the FDG PET/CT scan. Thereafter, the CT images of the FDG PET/CT scan were rigidly registered to the CT images of the ${ }^{89} \mathrm{Zr}$-cetuximab PET/CT scans. The tumour delineations were copied onto the ${ }^{89} \mathrm{Zr}$-cetuximab scan. All registrations and propagated delineations were visually checked and no registration difficulties were observed. The aortic arch was contoured for assessment of unspecific background uptake of the tracer.

\section{Quantification of PET tracer uptake}

PET/CT images were analysed using in-house developed Matlab-based software (The MathWorks Inc., Natick, MA). For the FDG PET/CT scan and ${ }^{89} \mathrm{Zr}$-cetuximab $\mathrm{PET} / \mathrm{CT}$ scans, tracer uptake was quantified using standardized uptake values (SUV) normalized to body weight. The mean uptake $\left(\mathrm{SUV}_{\text {mean }}\right)$, maximum uptake $\left(\mathrm{SUV}_{\max }\right)$, and peak uptake $\left(S U V_{\text {peak }}\right)$ were assessed inside the delineated tumour sites. $S U V_{\text {peak }}$ is defined as the mean SUV in a 3D sphere with a diameter of $1.2 \mathrm{~cm}$ centred at the 
tumour location with the highest activity. For the primary tumour, the GTV was used as region of interest. For the smaller lymph nodes, the clinical target volume $\left(\mathrm{CTV}_{\mathrm{ln}}\right)$ was used as region of interest, which consisted of an isotropic $5 \mathrm{~mm}$ extension of the $G_{T V}$..$S U V_{\max }$ and $S U V_{\text {peak }}$ were calculated for the lymph nodes. The average uptake in the aortic arch was calculated and the tumour-to-background ratio (TBR), as defined as the $\mathrm{SUV}_{\text {peak }}$ in the tumour divided by $\mathrm{SUV}_{\text {mean }}$ of the aorta, was determined. To determine the optimal ${ }^{89} \mathrm{Zr}$-cetuximab scan time point, we compared the contrast between the tumour and background activity for the first and second scan, and selected the time point with the highest ${ }^{89} \mathrm{Zr}$-cetuximab TBR.

The stability of the ${ }^{89} \mathrm{Zr}$-cetuximab uptake patterns were compared between the two scans. The second scan was registered to the first scan using a rigid registration and the GTV contours of the primary tumours were copied from the planning CT to the first ${ }^{89} \mathrm{Zr}$-cetuximab scan. All registrations were visually checked and no registration problems were observed. A voxel-based correlation between the SUV values of the two scans was calculated.

Finally, ${ }^{89}$ Zr-cetuximab images were compared to FDG PET/CT images. The peak ${ }^{89} \mathrm{Zr}$-cetuximab uptake and peak FDG uptake for the primary tumour were compared. In addition, the location of the high uptake regions on the ${ }^{89} \mathrm{Zr}$-cetuximab and FDG PET/CT scans were compared. For both the first and second ${ }^{89} \mathrm{Zr}$-cetuximab scan, high uptake regions were defined as the volume with a TBR above 1.2 or 1.4. Two cutoff values were used because it is still not well defined which cutoff value best quantifies significant ${ }^{89} \mathrm{Zr}$-cetuximab uptake. For the FDG PET/CT scans voxels with a SUV above $50 \%$ of the $\mathrm{SUV}_{\max }$ were defined as high uptake region. The overlap between the different volumes was assessed using a DICE similarity score, defined as twice the intersecting volume divided by the sum of both volumes.

\section{EGFR expression}

For all patients a pretreatment biopsy of the primary tumour was taken, as part of the regular diagnostic examination. Part of the tumour sample was archived in a paraffin block and later used for EGFR expression analysis. The archived samples were obtained from the Maastricht Pathology Tissue Collection (MPTC) and NKIAVL Core Facility Molecular Pathology \& Biobanking (CFMPB). Collection, storage and use of tissue and patient data were performed in agreement with the "Code for Proper Secondary Use of Human Tissue in the Netherlands". The EGFR expression assessment was performed with an EGFR pharmDx qualitative immunohistochemical kit, consisting of two antibodies (Novocastra and Dako, Denmark). All samples were analysed on the same day in the same lab. EGFR staining intensity was analysed using a light microscope. The percentages of cells with weak, moderate and strong 
membranous EGFR staining were scored. An EGFR immunohistochemistry (IHC) score, between 0-300, was calculated according to the formula: EGFR IHC score $=1 \times$ $\%$ cells weak staining $+2 \times \%$ cells moderate staining $+3 \times \%$ cells strong staining [30]. Tumours with an IHC score $<200$ were classified as low EGFR expression; tumours with an IHC score $\geq 200$ as high EGFR expression. This division was based on results of the FLEX study [31]. The EGFR expression was correlated to the ${ }^{89} \mathrm{Zr}$-cetuximab imaging parameters. The EGFR low and EGFR high expression group were compared.

\section{Statistics}

To evaluate the optimal time point for ${ }^{89} \mathrm{Zr}$-cetuximab imaging, the TBR of the primary tumour on the first and second scan were compared using a paired student $t$-test. The Pearson correlation coefficient was used to calculate the correlation between the ${ }^{89} \mathrm{Zr}$ cetuximab TBR in the primary tumour and lymph nodes, to determine the spatial stability between the two ${ }^{89} \mathrm{Zr}$-cetuximab scans, and to determine the correlation between ${ }^{89} \mathrm{Zr}$-cetuximab and FDG peak uptake in the primary tumour. A MannWhitney $\mathrm{U}$ exact test was used to assess the ${ }^{89} \mathrm{Zr}$-cetuximab parameters between the EGFR high and low uptake groups. Results are presented as mean \pm one standard deviation and p-values $<0.05$ were considered statistically significant.

\section{Results}

The first 17 patients (12 males, 5 females; age range: 45-68 years) enrolled in the ARTFORCE study received ${ }^{89} \mathrm{Zr}$-cetuximab imaging and were analysed. After a minimum follow-up of two years, three patients presented with a locoregional recurrence and three patients developed metastases. (Supplementary Table S3.1). Average primary tumour volume was $41.7 \mathrm{~cm}^{3} \pm 24.7 \mathrm{~cm}^{3}$. Sixteen of the seventeen patients had regional lymph nodes metastases. Fifteen patients had ${ }^{89} \mathrm{Zr}$-cetuximab scans at two time points available for analysis. For two patients only the scan at the second time point could be used. One of those patients refused a scan and for the other patient a scan was excluded from analysis because the aortic arch was not in the field of view. Those two patients were excluded for the optimal timing and temporal stability analysis; the data was used for the other analyses. All patients underwent pretreatment FDG PET/CT scan. The patient and tumour characteristics are listed in Table 3.1.

Quantitative PET analysis showed a large interpatient variety of tracer uptake. For the first scan the $S U V_{\text {peak }}$ ranged from 2.5-6.2 between patients, $S_{U V} V_{\text {max }}$ from 2.8-7.9, $\mathrm{SUV}_{\text {mean }}$ from 1.8-4.0 and TBR from 0.7-2.1. For the second scan the $\mathrm{SUV}_{\text {peak }}$ ranged from 2.5-6.2, $\mathrm{SUV}_{\max }$ from 2.9-7.7, $\mathrm{SUV}_{\text {mean }}$ from 1.6-3.9 and, TBR from 1.0-2.6. 
Table 3.1. Patient characteristics.

\begin{tabular}{|c|c|c|c|c|c|c|c|c|c|c|}
\hline \multirow[b]{2}{*}{ Patient } & \multirow[b]{2}{*}{ Age } & \multirow[b]{2}{*}{ Sex } & \multirow{2}{*}{$\begin{array}{l}\text { Primary } \\
\text { tumour site }\end{array}$} & \multicolumn{3}{|c|}{ Tumour stage } & \multirow{2}{*}{$\begin{array}{l}\text { TNM group } \\
\text { staging }\end{array}$} & \multirow{2}{*}{$\begin{array}{l}\text { Primary tumour } \\
\text { volume }\left(\mathrm{cm}^{3}\right)\end{array}$} & \multirow{2}{*}{$\begin{array}{l}\text { HPV status } \\
\quad(\mathrm{p} 16)\end{array}$} & \multirow{2}{*}{$\begin{array}{c}\text { EGFR IHC } \\
\text { score }\end{array}$} \\
\hline & & & & $\mathrm{T}$ & $\mathrm{N}$ & M & & & & \\
\hline 1 & 61 & M & Oropharynx & T3 & N1 & M0 & III & 20 & + & 270 \\
\hline 2 & 65 & M & Oral cavity & $\mathrm{T} 4$ & N1 & M0 & IV & 54 & NA & 210 \\
\hline 3 & 55 & M & Oropharynx & $\mathrm{T} 4$ & $\mathrm{~N} 2 \mathrm{~b}$ & M0 & IV & 53 & - & 184 \\
\hline 4 & 56 & $\mathrm{~F}$ & Oropharynx & T3 & $\mathrm{N} 2 \mathrm{~b}$ & M0 & IV & 11 & + & 207 \\
\hline 5 & 66 & $\mathrm{~F}$ & Oropharynx & $\mathrm{T} 4 \mathrm{~b}$ & $\mathrm{~N} 2 \mathrm{~b}$ & M0 & III & 30 & - & 245 \\
\hline 6 & 45 & M & Oral cavity & $\mathrm{T} 4 \mathrm{a}$ & $\mathrm{N} 2 \mathrm{c}$ & M0 & II & 98 & NA & 210 \\
\hline 7 & 62 & M & Oropharynx & T3 & N3 & M0 & IV & 44 & + & 80 \\
\hline 8 & 57 & M & Oropharynx & T3 & No & M0 & III & 20 & - & 235 \\
\hline 9 & 68 & M & Hypopharynx & $\mathrm{T} 4$ & $\mathrm{~N} 2 \mathrm{~b}$ & M0 & IV & 41 & NA & 1 \\
\hline 10 & 63 & M & Oropharynx & $\mathrm{T} 4$ & $\mathrm{~N} 2 \mathrm{c}$ & M0 & IV & 42 & + & 10 \\
\hline 11 & 64 & M & Oral cavity & $\mathrm{T} 4$ & $\mathrm{~N} 2 \mathrm{~b}$ & M0 & IV & 78 & NA & 70 \\
\hline 12 & 60 & $\mathrm{~F}$ & Oral cavity & $\mathrm{T} 4$ & N1 & M0 & IV & 21 & NA & 212 \\
\hline 13 & 50 & M & Oral cavity & $\mathrm{T} 4$ & $\mathrm{~N} 2 \mathrm{~b}$ & M0 & IV & 76 & NA & 200 \\
\hline 14 & 55 & $\mathrm{~F}$ & Oropharynx & $\mathrm{T} 4$ & N1 & M0 & IV & 51 & + & 180 \\
\hline 15 & 68 & $\mathrm{~F}$ & Oropharynx & T3 & $\mathrm{N} 2 \mathrm{c}$ & M0 & IV & 30 & + & 225 \\
\hline 16 & 55 & M & Oropharynx & T3 & $\mathrm{N} 2 \mathrm{~b}$ & M0 & IV & 10 & - & 5 \\
\hline 17 & 67 & M & Hypopharynx & T3 & $\mathrm{N} 2 \mathrm{c}$ & M0 & IV & 29 & NA & 285 \\
\hline
\end{tabular}

Abbreviations: $\mathrm{M}=$ male, $\mathrm{F}=$ female, $\mathrm{NA}=$ not assessed. $\mathrm{HPV}$ status was assessed with p16 immunohistochemistry.

Table 3.2. ${ }^{89} \mathrm{Zr}$-cetuximab uptake on scan 1 and 2 , the difference of scan 2 compared to scan 1 , and FDG PET uptake.

\begin{tabular}{llccrr}
\hline & & $\begin{array}{c}{ }^{89} \text { Zr-cetuximab } \\
\text { scan 1 }\end{array}$ & $\begin{array}{c}{ }^{89} \text { Zr-cetuximab } \\
\text { scan 2 }\end{array}$ & $\begin{array}{c}\text { Difference } \\
{ }^{89} \text { Zr-cetuximab (\%) }\end{array}$ & \multicolumn{1}{c}{ FDG } \\
\hline \multirow{2}{*}{ Primary tumour } & SUV $_{\text {peak }}$ & $4.1 \pm 1.2$ & $4.0 \pm 1.2$ & $-1.3 \pm 9.4$ & $14.3 \pm 6.9$ \\
& SUV $_{\text {max }}$ & $5.0 \pm 1.8$ & $4.9 \pm 1.6$ & $1.5 \pm 12.1$ & $17.6 \pm 7.8$ \\
& SUV $_{\text {mean }}$ & $2.6 \pm 0.7$ & $2.6 \pm 0.7$ & $-0.6 \pm 11.9$ & $6.4 \pm 2.8$ \\
& TBR & $1.2 \pm 0.4$ & $1.7 \pm 0.6$ & $49.1 \pm 28.1$ & \\
Lymph nodes & SUV & $3.4 \pm 1.0$ & $3.4 \pm 1.2$ & $-7.6 \pm 12.9$ & $8.3 \pm 5.2$ \\
& SUV $_{\text {max }}$ & $4.1 \pm 1.2$ & $4.1 \pm 1.6$ & $-3.5 \pm 12.7$ & $10.9 \pm 5.6$ \\
& TBR & $0.9 \pm 0.2$ & $1.4 \pm 0.5$ & $43.3 \pm 35.0$ & \\
\hline \multirow{2}{*}{ Aortic Arch } & SUV & $3.6 \pm 0.9$ & $2.5 \pm 0.9$ & $-31.7 \pm 13.4$ & \\
\hline
\end{tabular}

Average $\mathrm{SUV}_{\text {peak }}$, $\mathrm{SUV}_{\text {max }}, \mathrm{SUV}_{\text {mean }}$, and TBR values for the primary tumour and the lymph nodes, for the first and second ${ }^{89} \mathrm{Zr}$-cetuximab PET/CT scan are shown in Table 3.2. The imaging features for the individual patients are shown in Supplementary Table S3.1 for the primary tumour, and in Supplementary Table S3.2 for the lymph nodes. The ${ }^{89} \mathrm{Zr}$-cetuximab TBR in the primary tumour was for all patients higher on the second scan compared to the first scan. The TBR increased on average with $49 \% \pm 28 \%(\mathrm{p}<0.01)$, indicating an improved imaging quantification profile at the later time points. The two ${ }^{89} \mathrm{Zr}$-cetuximab scans of an example patient are shown in Figure 3.1. In Figure 3.2 the TBR is plotted as function of the number of days after ${ }^{89} \mathrm{Zr}$-cetuximab administration for the individual patients.

In patients with a high ${ }^{89} \mathrm{Zr}$-cetuximab uptake in the primary tumour, in general 

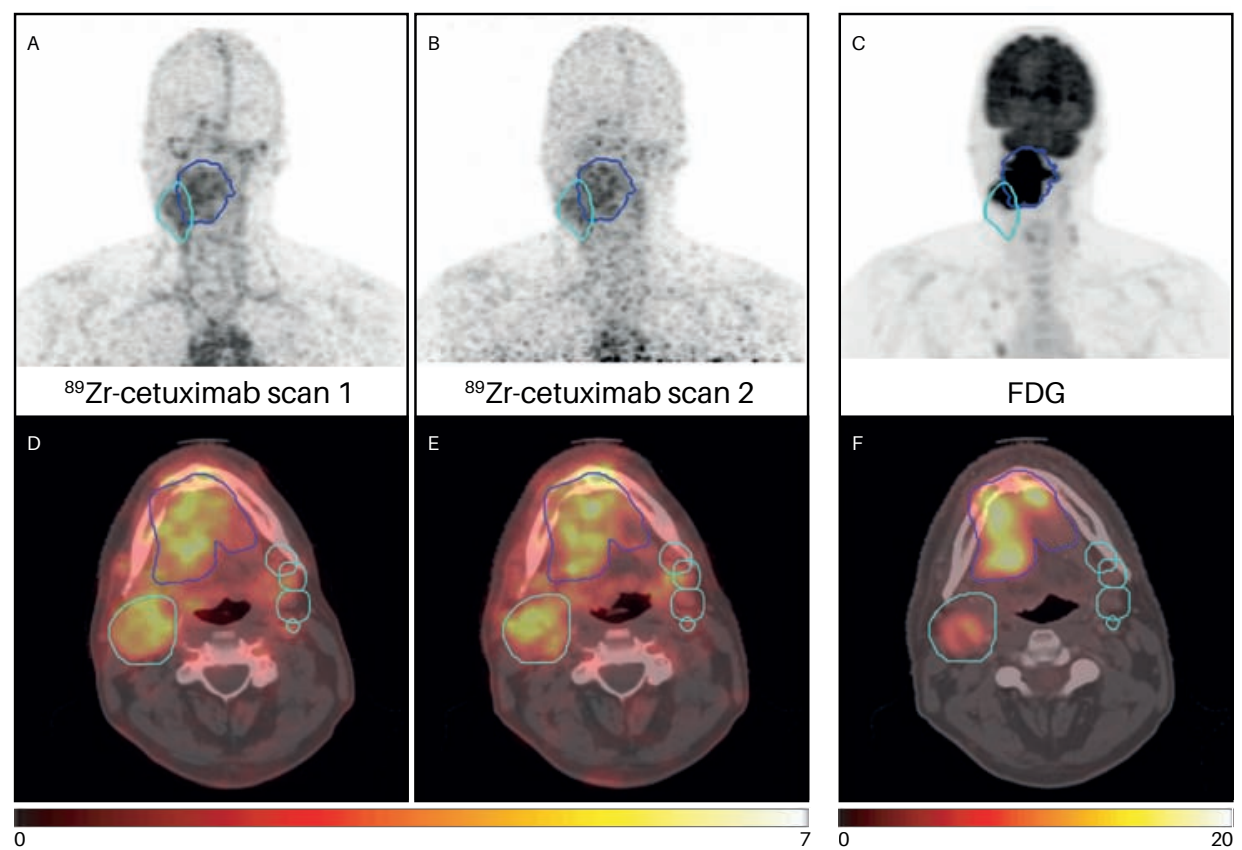

Figure 3.1. PET maximum intensity projections (MIP) (top row) and fused PET/CT images showing PET uptake in SUV (bottom row) of the two ${ }^{89} \mathrm{Zr}$-cetuximab scans and the FDG PET/CT (patient 6). The GTV for the primary tumour is depicted in blue; the CTV for the lymph nodes in cyan. Only the largest lymph node is displayed in the MIP.

also an elevated maximum uptake in the lymph nodes was observed, as shown in Supplementary Figure S3.1. For the second scan a strong, significant correlation was found between the TBR in the lymph nodes and primary tumour $(r=0.76, p<0.01)$.

The voxel-based comparison between the two ${ }^{89} \mathrm{Zr}$-cetuximab uptake patterns, showed correlation coefficients ranging from 0.18-0.86, see Supplementary Table S3.1. The patients with a low ${ }^{89} \mathrm{Zr}$-cetuximab uptake $(\mathrm{TBR}<1.2)$ had correlation coefficients of $0.18,0.20$ and 0.66 . Excluding these patients with low uptake levels, resulted for the remaining 13 patients in an average spatial correlation of $0.68 \pm 0.11$ between the two scans.

In Figure 3.1 (C and F) FDG PET/CT scan is displayed. No correlation was found between the FDG SUV peak and ${ }^{89} \mathrm{Zr}$-cetuximab $\mathrm{SUV}_{\text {peak }}$ in the primary tumour, for the first ( $\mathrm{r}=0.11, \mathrm{p}=0.69$ ) or second ${ }^{89} \mathrm{Zr}$-cetuximab PET/CT scan $(\mathrm{r}=0.46, \mathrm{p}=0.07$ ). Comparison of the high spatial uptake regions showed only minor overlap between high ${ }^{89} \mathrm{Zr}$-cetuximab uptake regions (TBR $>1.2$ or 1.4) and high FDG uptake regions $\left(>50 \%\right.$ of $S U V_{\text {max }}$ ). The volumes of the high uptake regions and DICE scores are shown in Table 3.3. 

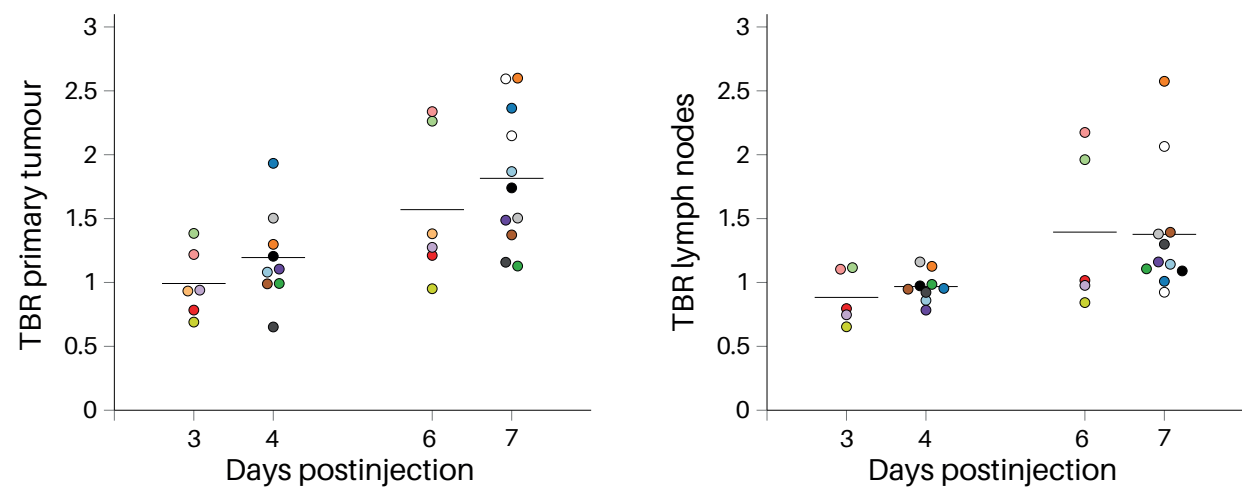

Figure 3.2. The tumour-to-background ratio (TBR) of ${ }^{89} \mathrm{Zr}$-cetuximab in the primary tumour (left), and the maximum TBR in the lymph nodes (right) plotted as a function of the number of days between ${ }^{89} \mathrm{Zr}$-cetuximab administration and PET/CT imaging. The bars depict the mean uptake for the individual scan points. Each patient is depicted with a different colour. Two patients (white marks) were only scanned on one day.

Table 3.3. Volumes of high uptake regions of ${ }^{89} \mathrm{Zr}$-cetuximab and FDG PET. Overlap comparison between high ${ }^{89} \mathrm{Zr}$-cetuximab and high FDG PET uptake regions using the DICE similarity coefficient.

\begin{tabular}{|c|c|c|c|c|c|c|}
\hline & \multicolumn{3}{|c|}{${ }^{89} \mathrm{Zr}$-cetuximab scan 1} & \multicolumn{3}{|c|}{${ }^{89} \mathrm{Zr}$-cetuximab scan 2} \\
\hline & ${ }^{89}$ Zr-cetux & ${ }^{89} \mathrm{Zr}$-cetux & FDG $>50 \%$ & ${ }^{89} \mathrm{Zr}$-cetux & ${ }^{89} \mathrm{Zr}$-cetux & FDG $>50 \%$ \\
\hline & $\mathrm{TBR}>1.2$ & $\mathrm{TBR}>1.4$ & $\mathrm{SUV}_{\max }$ & $\mathrm{TBR}>1.2$ & $\mathrm{TBR}>1.4$ & $\mathrm{SUV}_{\max }$ \\
\hline Volume $\left(\mathrm{cm}^{3}\right)$ & $20.7 \pm 6.4$ & $7.2 \pm 6.6$ & $28.4 \pm 11.4$ & $21.7 \pm 4.7$ & $6.6 \pm 4.3$ & $28.3 \pm 11.5$ \\
\hline DICE & $0.4 \pm 0.2$ & $0.2 \pm 0.2$ & & $0.4 \pm 0.1$ & $0.2 \pm 0.1$ & \\
\hline
\end{tabular}

The EGFR IHC scores showed seven tumours (41\%) with a low EGFR expression, IHC $<200$, (IHC: $76 \pm 79$ ) and ten tumours (59 \%) with a high expression, IHC $\geq 200$, (IHC: $230 \pm 29$ ). Based on the second ${ }^{89} \mathrm{Zr}$-cetuximab PET/CT scan, the $\mathrm{SUV}_{\text {mean }}$ was $2.1 \pm 0.5$ and $3.0 \pm 0.6$ for the low and high EGFR expressing group respectively. The $\mathrm{SUV}_{\text {peak }}$ was $3.2 \pm 0.6$ and $4.7 \pm 1.1$ respectively, the $\mathrm{TBR}_{\text {mean }} 1.0 \pm 0.3$ and $1.2 \pm 0.3$, and the $\mathrm{TBR}_{\text {peak }} 1.6 \pm 0.6$ and $1.8 \pm 0.5$, where $\mathrm{TBR}_{\text {mean }}$ and $\mathrm{TBR}_{\text {peak }}$ are the $\mathrm{SUV}_{\text {mean }}$ and $\mathrm{SUV}_{\text {peak }}$ divided by the background uptake. The $\mathrm{SUV}_{\text {mean }}(\mathrm{p}<0.01)$ and $\mathrm{SUV}_{\text {peak }}$ ( $\mathrm{p}<0.01$ ) were significantly different between the low and high EGFR expression groups, however for the $\mathrm{TBR}_{\text {mean }}(\mathrm{p}=0.315)$ and $\mathrm{TBR}_{\text {peak }}(\mathrm{p}=0.417)$ no statistical significance was observed. In the group with a low EGFR expression, three out of seven (42\%) patients had a high ${ }^{89} \mathrm{Zr}$-cetuximab TBR $\left(\mathrm{TBR}_{\text {peak }}>1.4\right)$; in the group with high EGFR expression seven out of ten (70\%) patients had high uptake $\left(\mathrm{TBR}_{\text {peak }}>1.4\right)$. In Figure 3.3 the PET parameters as a function of EGFR IHC scores are shown. 

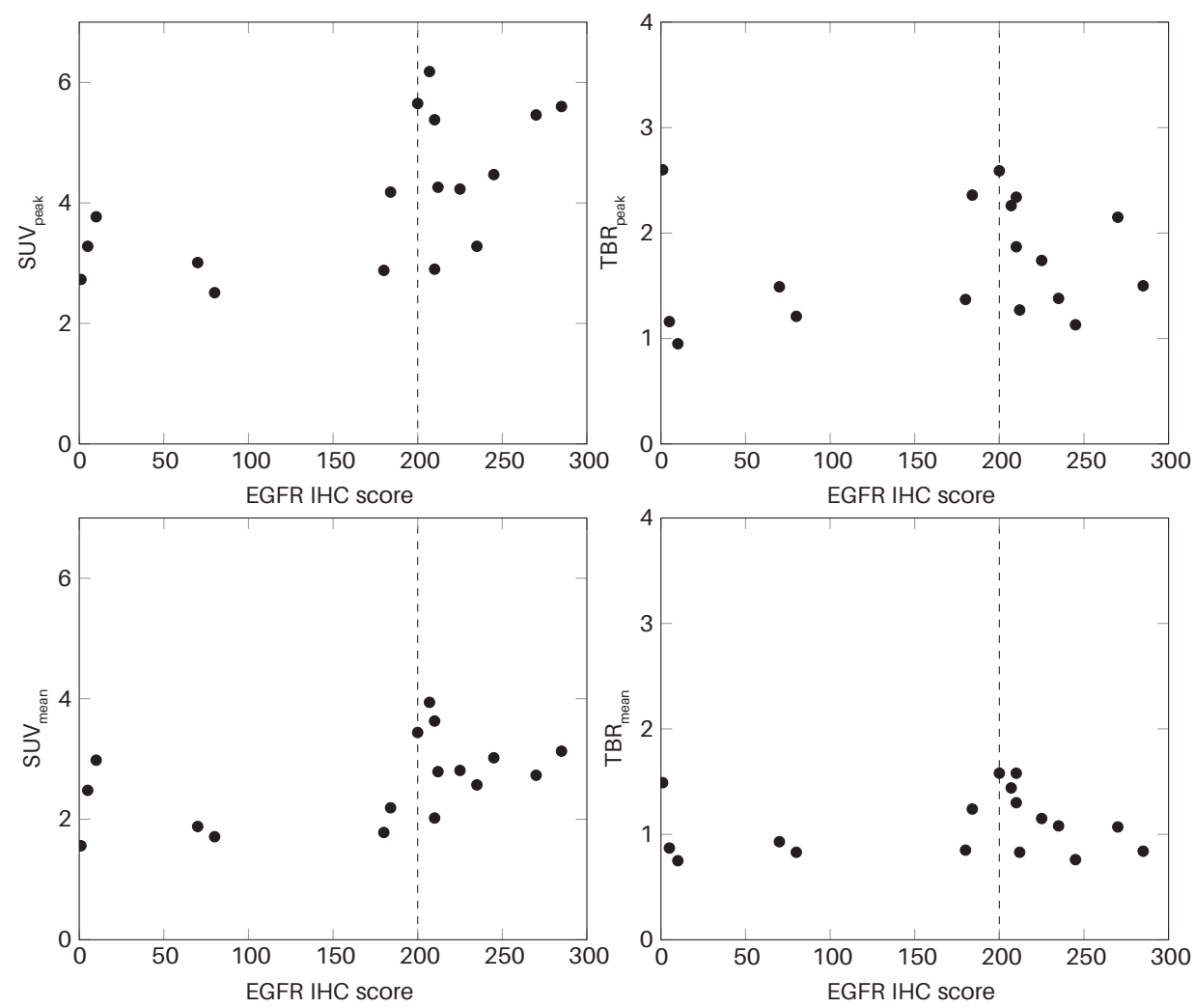

Figure 3.3. Correlation between the EGFR immunohistochemistry (IHC) score and the ${ }^{89} \mathrm{Zr}$ cetuximab peak and mean TBR, and peak and mean SUV in the primary tumour. The PET parameters are calculated for the second ${ }^{89} \mathrm{Zr}$-cetuximab scan (6 or 7 days postinjection). An EGFR IHC score $\geq 200$ (dashed line) is classified as high EGFR expression, an IHC score $<200$ as low EGFR expression.

\section{Discussion}

This study analysed ${ }^{89} \mathrm{Zr}$-cetuximab PET/CT imaging at two time points before radiotherapy treatment, to determine the optimal timing of ${ }^{89} \mathrm{Zr}$-cetuximab imaging, the spatial stability of the ${ }^{89} \mathrm{Zr}$-cetuximab uptake patterns, and the uptake in the primary tumour compared to the lymph nodes to allow future use in treatment selection. Furthermore, ${ }^{89} \mathrm{Zr}$-cetuximab uptake was compared with EGFR expression and metabolic activity as determined by FDG PET/CT.

The later ${ }^{89} \mathrm{Zr}$-cetuximab imaging time points were associated with increased tumour-to-background ratios in all patients, therefore, imaging at 6 or 7 days postinjection is recommended for future studies. This is in agreement with the results in advanced colorectal cancer [32], where also 6 days postinjection was described as 
optimal imaging time point. The imaging time point did not influence the average peak or maximum uptake; the improvement in TBR between the time points is due to a decrease in background activity, see Supplementary Table S3.3. The voxel-based comparison between the two ${ }^{89} \mathrm{Zr}$-cetuximab scans showed that there is a correlation for patients with sufficient uptake of the tracer. The correlation is moderate however, indicating that there is a minor change in spatial uptake patterns over time.

As anticipated, a large variation in ${ }^{89} \mathrm{Zr}$-cetuximab uptake was found between patients. The TBR on the second scan ranged from the background level (TBR around 1.0) to a TBR of 2.6 times the background level. This interpatient variety could possibly be exploited and used to select tumours that can be targeted by the monoclonal antibody to ultimately predict treatment outcome. However, more research is required to determine which of the calculated measures $\left(\mathrm{SUV}_{\text {mean }}, \mathrm{SUV}_{\text {peak }}\right.$, $S U V_{\max }, T B R$ ) best reflects the accessibility of the drug to the tumour. To be able to use ${ }^{89} \mathrm{Zr}$-cetuximab PET/CT imaging for patient selection, the antibody uptake should be related to treatment outcome and an appropriate measure for differentiating the responders and non-responders should be determined. The studied group of patients was unfortunately too small and the received treatments too heterogeneous to link treatment outcome to ${ }^{89} \mathrm{Zr}$-cetuximab uptake. A sample of more than 17 patients is needed to define such a measure.

The exploratory analysis comparing high uptake regions on the ${ }^{89} \mathrm{Zr}$-cetuximab PET/CT images with the high uptake regions on FDG images revealed only minor overlap (DICE $<0.6$ for TBR > 1.2). A correlation between the two tracers could be hypothesized given that EGFR influences proliferation and thereby glucose metabolism. However, many factors other than EGFR expression contribute to a difference in metabolism. Studies linking proliferation to FDG uptake have shown conflicting results $[33,34]$.

A significant difference in ${ }^{89} \mathrm{Zr}$-cetuximab SUV was found between the groups with a high and a low EGFR expression. The tumours with a high expression had on average a higher ${ }^{89} \mathrm{Zr}$-cetuximab $\mathrm{SUV}_{\text {mean }}$ and $\mathrm{SUV}_{\text {peak }}$. However, when we compared the TBR between the two groups, no significant difference was observed. In tumours lacking EGFR expression, response to the targeted drug was unexpected, while in tumours with an EGFR overexpression, the accessibility of the tumour was hypothesized to be a determining factor in drug uptake. As expected, in the tumours with high EGFR expression a mix of low and high ${ }^{89} \mathrm{Zr}$-cetuximab uptake was observed. Remarkably, high ${ }^{89} \mathrm{Zr}$-cetuximab PET uptake was also observed in the low EGFR expression group. The patient with the lowest EGFR IHC score had the overall highest ${ }^{89} \mathrm{Zr}$ cetuximab TBR. A limitation of this kind of analysis and a possible explanation for this remarkable result is the fact that EGFR expression was only determined for one sample which might not represent the whole tumour [35]. In contrast to a 
single biopsy, ${ }^{89} \mathrm{Zr}$-cetuximab imaging can give a $3 \mathrm{D}$ uptake pattern of the entire tumour. Another limitation of a single biopsy is the questionable reproducibility of EGFR staining. Interobserver variability, differences in tissue fixation techniques, and increased storage time of the samples could negatively influence reproducibility. For example, Chung et al. [36] found that cetuximab shows activity in tumours that do not express EGFR and concluded that EGFR determined by immunohistochemistry might not reflect the tumour biology.

A high correlation was observed between the ${ }^{89} \mathrm{Zr}$-cetuximab uptake in the primary tumour and the lymph nodes. This might indicate that uptake of the drug is mainly determined by intrinsic characteristics of the tumour cells. Interestingly, the Bonner trial [6] showed in an exploratory subgroup analysis an increased benefit for addition of cetuximab to radiotherapy over radiotherapy alone for patients with nodal involvement, while there was no benefit for patients without nodal involvement. Our data shows that a patient with uptake of cetuximab in the primary tumour will most likely also have cetuximab accumulating in the lymph nodes.

A limitation of this study is the use of different PET scan settings, e.g. voxel size, slice thickness, scan time, and various PET reconstructions algorithms. This complicates quantitative analysis and comparison between patients. After inclusion of the first patients in this study, Makris et al. [28] published work highlighting the importance of harmonization of scan protocols. These suggested scan protocols were adopted. Thereafter, their recommendations regarding image analysis were followed to improve the conformity between scans. For example, scans acquired on a Philips scanner were additionally smoothed and $S_{U V} V_{\text {peak }}$ was used as measure of tracer uptake since this parameter is less susceptible to noise.

Other factors that could have influenced the imaging results are the cold loading dose and the extra margin for the lymph nodes. A loading dose was used to prevent all labelled antibody going to the liver. As a proof of principle, it was shown before that without a loading dose less than $10 \%$ of the injected ${ }^{89} \mathrm{Zr}$-cetuximab dose was available in the blood, while after a loading dose of $500 \mathrm{mg} / \mathrm{m}^{2} 80 \%$ of the injected tracer was detected in the blood and available for tumour targeting [32]. For this study the recommended therapeutic dose of cetuximab $\left(400 \mathrm{mg} / \mathrm{m}^{2}\right)$ was used as a loading dose to best represent the clinical situation and to visualize how much cetuximab would reach the tumour during treatment. For the lymph nodes, we decided to use the CTV as region of interest. This additional margin, that was added to compensate for small registration uncertainties, would influence the $S U V_{\text {mean }}$ of the region. Therefore, only the $S U V_{\text {max }}$ and $S U V_{\text {peak }}$ were calculated for the lymph nodes. Smaller lymph nodes might suffer from underestimation of the uptake due to partial volume effects.

The ARTFORCE trial was designed to select the most effective treatment, cisplatin or cetuximab, for individual patients. The original design consisted of four treatment 
arms (two with cisplatin and two with cetuximab). All treatment arms were preceded by an ${ }^{89} \mathrm{Zr}$-cetuximab pretreatment imaging step which was solely used for research purposes. The use of a long-lived positron emitter complicated procedures for the radiotherapy departments, patients were confronted with an extra radiation burden (0.61 mSv/MBq [37]) and additional guidelines had to be followed by the patient during two weeks after injection to limit radiation exposure to others. The resulting slow accrual in combination with discontinuation of the funding of cetuximab resulted in an amendment of the trial excluding ${ }^{89} \mathrm{Zr}$-cetuximab PET/CT imaging and the cetuximab treatment arms. The imaging results of all patients undergoing the ${ }^{89} \mathrm{Zr}$ cetuximab PET/CT imaging were presented in this study.

In conclusion, the PET tracer ${ }^{89} \mathrm{Zr}$-cetuximab showed a large variation in ${ }^{89} \mathrm{Zr}$ cetuximab tumour-to-background ratio between patients. This interpatient variety could possibly be exploited and used to select tumours that can be targeted by the monoclonal antibody to ultimately predict treatment outcome. ${ }^{89} \mathrm{Zr}$-cetuximab imaging provides additional information about the accessibility of the drug into the tumour which is not provided by FDG PET or EGFR expression. Validation of the predictive value is recommended with scans acquired 6 to 7 days postinjection to obtain high tumour-to-background uptake levels. For future studies a trial design should be chosen that incentivizes patients to participate, e.g. by linking research and therapeutic consequences.

\section{Acknowledgements}

Authors acknowledge financial support from ERC advanced grant (ERC-ADG-2015, $\mathrm{n}^{\circ} 694812$ - Hypoximmuno) and the QuIC-ConCePT project, which is partly funded by EFPI A companies and the Innovative Medicine Initiative Joint Undertaking (IMI JU) under Grant Agreement No. 115151. This research is also supported by the Dutch technology Foundation STW (grant n 10696 DuCAT \& n P14-19 Radiomics STRaTegy), which is the applied science division of NWO, and the Technology Programme of the Ministry of Economic Affairs. Authors also acknowledge financial support from the EU 7th framework program (ARTFORCE - $\mathrm{n}^{\circ}$ 257144, REQUITE - $\mathrm{n}^{\circ}$ 601826), SME Phase 2 (EU proposal 673780 - RAIL), EUROSTARS (DART), the European Program H2020-2015-17 (BD2Decide - PHC30-689715 and ImmunoSABR $-\mathrm{n}^{\circ}$ 733008), Kankeronderzoekfonds Limburg from the Health Foundation Limburg, Alpe 'HuZes-KWF (DESIGN) and the Dutch Cancer Society (KWF UM 2011-5020, KWF UM 2009-4454, KWF MAC 2013-6425, KWF MAC 2013-6089, KWF 2015-7635). Authors acknowledge MERCK for supplying cetuximab and Anisha Gogineni for editing. 


\section{References}

1. Argiris, A., Karamouzis, M. V., Raben, D. \& Ferris, R. L. Head and neck cancer. Lancet 371, 1695-709 (2008).

2. Pulte, D. \& Brenner, H. Changes in survival in head and neck cancers in the late 20th and early 21st century: a period analysis. Oncologist 15, 994-1001 (2010).

3. Pignon, J. P., le Maître, A., Maillard, E. \& Bourhis, J. Meta-analysis of chemotherapy in head and neck cancer (MACH-NC): an update on 93 randomised trials and 17,346 patients. Radiother Oncol 92, 4-14 (2009).

4. Pignon, J. P., le Maître, A. \& Bourhis, J. Meta-analyses of chemotherapy in head and neck cancer (MACH-NC): an update. Int J Radiat Oncol 69, S112-4 (2007).

5. Blanchard, P., Baujat, B., Holostenco, V., et al. Meta-analysis of chemotherapy in head and neck cancer (MACH-NC): A comprehensive analysis by tumour site. Radiother Oncol 100, 33-40 (2011).

6. Bonner, J. A., Harari, P. M., Giralt, J., et al. Radiotherapy plus cetuximab for locoregionally advanced head and neck cancer: 5 -year survival data from a phase 3 randomised trial, and relation between cetuximab-induced rash and survival. Lancet Oncol 11, 21-8 (2010).

7. Ang, K. K., Berkey, B. A., Tu, X., et al. Impact of epidermal growth factor receptor expression on survival and pattern of relapse in patients with advanced head and neck carcinoma. Cancer Res 62, 7350-6 (2002).

8. Herbst, R. S. Review of epidermal growth factor receptor biology. Int J Radiat Oncol 59, 21-6 (2004).

9. Aerts, H. J. W. L., Dubois, L., Hackeng, T. M., et al. Development and evaluation of a cetuximab-based imaging probe to target EGFR and EGFRvIII. Radiother Oncol 83, 326-32 (2007).

10. Jutten, B., Dubois, L., Li, Y., et al. Binding of cetuximab to the EGFRvIII deletion mutant and its biological consequences in malignant glioma cells. Radiother Oncol 92, 393-8 (2009).

11. Jutten, B. \& Rouschop, K. M. EGFR signaling and autophagy dependence for growth, survival, and therapy resistance. Cell Cycle 13, 42-51 (2014).

12. Theys, J., Jutten, B., Dubois, L., et al. The deletion mutant EGFRvIII significantly contributes to stress resistance typical for the tumour microenvironment. Radiother Oncol 92, 399-404 (2009). 
13. Caudell, J. J., Sawrie, S. M., Spencer, S. A., et al. Locoregionally advanced head and neck cancer treated with primary radiotherapy: a comparison of the addition of cetuximab or chemotherapy and the impact of protocol treatment. Int J Radiat Oncol 71, 676-81 (2008).

14. Petrelli, F., Coinu, A., Riboldi, V., et al. Concomitant platinum-based chemotherapy or cetuximab with radiotherapy for locally advanced head and neck cancer: a systematic review and meta-analysis of published studies. Oral Oncol 50, 1041-8 (2014).

15. Ang, K. K., Zhang, Q., Rosenthal, D. I., et al. Randomized phase III trial of concurrent accelerated radiation plus cisplatin with or without cetuximab for stage III to IV head and neck carcinoma: RTOG 0522. J Clin Oncol 32, 2940-50 (2014).

16. Bradley, J. D., Paulus, R., Komaki, R., et al. Standard-dose versus high-dose conformal radiotherapy with concurrent and consolidation carboplatin plus paclitaxel with or without cetuximab for patients with stage IIIA or IIIB nonsmall-cell lung cancer (RTOG 0617): a randomised, two-by-two factorial phase 3 study. Lancet Oncol 16, 187-99 (2015).

17. Ciardiello, F. \& Tortora, G. EGFR antagonists in cancer treatment. New Engl J Med 358, 1160-74 (2008).

18. Braig, F., Voigtlaender, M., Schieferdecker, A., et al. Liquid biopsy monitoring uncovers acquired RAS-mediated resistance to cetuximab in a substantial proportion of patients with head and neck squamous cell carcinoma. Oncotarget 7, 42988-95 (2016).

19. Aerts, H. J. W. L., Dubois, L., Perk, L., et al. Disparity between in vivo EGFR expression and ${ }^{89} \mathrm{Zr}$-labeled cetuximab uptake assessed with PET. J Nucl Med 50, 123-31 (2009).

20. Lambin, P., van Stiphout, R. G. P. M., Starmans, M. H. W., et al. Predicting outcomes in radiation oncology-multifactorial decision support systems. Nat Rev Clin Oncol 10, 27-40 (2013).

21. Lambin, P., Zindler, J., Vanneste, B. G. L., et al. Decision support systems for personalized and participative radiation oncology. Adv Drug Deliv Rev 109, 131 53 (2016).

22. Fracasso, P. M., Burris III, H., Arquette, M. A., et al. A phase 1 escalating single-dose and weekly fixed-dose study of cetuximab: pharmacokinetic and pharmacodynamic rationale for dosing. Clin Cancer Res 13, 986-93 (2007). 
23. Perk, L. R., Visser, G. W. M., Vosjan, M. J. W. D., et al. ${ }^{89} \mathrm{Zr}$ as a PET surrogate radioisotope for scouting biodistribution of the therapeutic radiometals ${ }^{90} \mathrm{Y}$ and ${ }^{177} \mathrm{Lu}$ in tumor-bearing nude mice after coupling to the internalizing antibody cetuximab. J Nucl Med 46, 1898-906 (2005).

24. Van Loon, J., Even, A. J. G., Aerts, H. J. W. L., et al. PET imaging of zirconium89 labelled cetuximab: a phase I trial in patients with head and neck and lung cancer. Radiother Oncol 122, 267-73 (2017).

25. Heukelom, J., Hamming, O., Bartelink, H., et al. Adaptive and innovative Radiation Treatment FOR improving Cancer treatment outcomE (ARTFORCE); a randomized controlled phase II trial for individualized treatment of head and neck cancer. BMC Cancer 13, 84 (2013).

26. Boellaard, R., O'Doherty, M. J., Weber, W. A., et al. FDG PET and PET/CT: EANM procedure guidelines for tumour PET imaging: version 1.0. Eur J Nucl Med Mol I 37, 181-200 (2010).

27. Verel, I., Visser, G. W. M., Boellaard, R., Stigter-van Walsum, M., Snow, G. B. \& van Dongen, G. A. M. S. ${ }^{89} \mathrm{Zr}$ immuno-PET: comprehensive procedures for the production of ${ }^{89} \mathrm{Zr}$-labeled monoclonal antibodies. J Nucl Med 44, 1271-81 (2003).

28. Makris, N. E., Boellaard, R., Visser, E. P., et al. Multicenter harmonization of ${ }^{89} \mathrm{Zr}$ PET/CT performance. J Nucl Med 55, 264-7 (2014).

29. Even, A. J. G., Hamming-Vrieze, O., van Elmpt, W., et al. Cancer Data Data from: Quantitative assessment of zirconium-89 labeled cetuximab using PET/CT imaging in patients with advanced head and neck cancer: a theragnostic approach. http://dx.doi.org/10.17195/candat.2016.11.1.

30. Lee, H. J., Xu, X., Choe, G., et al. Protein overexpression and gene amplification of epidermal growth factor receptor in nonsmall cell lung carcinomas: comparison of four commercially available antibodies by immunohistochemistry and fluorescence in situ hybridization study. Lung Cancer 68, 375-82 (2010).

31. Pirker, R., Pereira, J. R., von Pawel, J., et al. EGFR expression as a predictor of survival for first-line chemotherapy plus cetuximab in patients with advanced non-small-cell lung cancer: analysis of data from the phase 3 FLEX study. Lancet Oncol 13, 33-42 (2012).

32. Menke-van der Houven van Oordt, C. W., Gootjes, E. C., Huisman, M. C., et al. ${ }^{89} \mathrm{Zr}$-cetuximab PET imaging in patients with advanced colorectal cancer. Oncotarget 6, 30384-93 (2015). 
33. Van Baardwijk, A., Dooms, C., van Suylen, R. J., et al. The maximum uptake of ${ }^{18} \mathrm{~F}$ deoxyglucose on positron emission tomography scan correlates with survival, hypoxia inducible factor-1alpha and GLUT-1 in non-small cell lung cancer. Eur J Cancer 43, 1392-8 (2007).

34. Sauter, A. W., Winterstein, S., Spira, D., et al. Multifunctional profiling of nonsmall cell lung cancer using ${ }^{18} \mathrm{~F}-\mathrm{FDG}$ PET/CT and volume perfusion CT. J Nucl Med 53, 521-9 (2012).

35. Iakovlev, V. V., Pintilie, M., Morrison, A., Fyles, A. W., Hill, R. P. \& Hedley, D. W. Effect of distributional heterogeneity on the analysis of tumor hypoxia based on carbonic anhydrase IX. Lab Invest 87, 1206-17 (2007).

36. Chung, K. Y., Shia, J., Kemeny, N. E., et al. Cetuximab shows activity in colorectal cancer patients with tumors that do not express the epidermal growth factor receptor by immunohistochemistry. J Clin Oncol 23, 1803-10 (2005).

37. Makris, N. E., Boellaard, R., van Lingen, A., et al. PET/CT-derived whole-body and bone marrow dosimetry of ${ }^{89} \mathrm{Zr}$-cetuximab. J Nucl Med 56, 249-54 (2015). 


\section{Supplementary data}

\section{Labelling of ${ }^{89} \mathrm{Zr}$-cetuximab}

${ }^{89} \mathrm{Zr}$-cetuximab is produced in compliance with the current Good Manufacturing Practice at the VU University Medical Center. The radiochemical purity was measured by TLC and SEC-HPLC and was $98.4 \% \pm 0.4 \%$ and $98.5 \% \pm 1.4 \%$, relatively. The mean immunoreactive fraction was $96.6 \% \pm 2.3 \%$. The filter integrity was on average $3.7 \mathrm{bar} \pm 0.5$ bar. The edotoxin content was for each batch smaller than $0.3 \mathrm{EU} / \mathrm{ml}$.

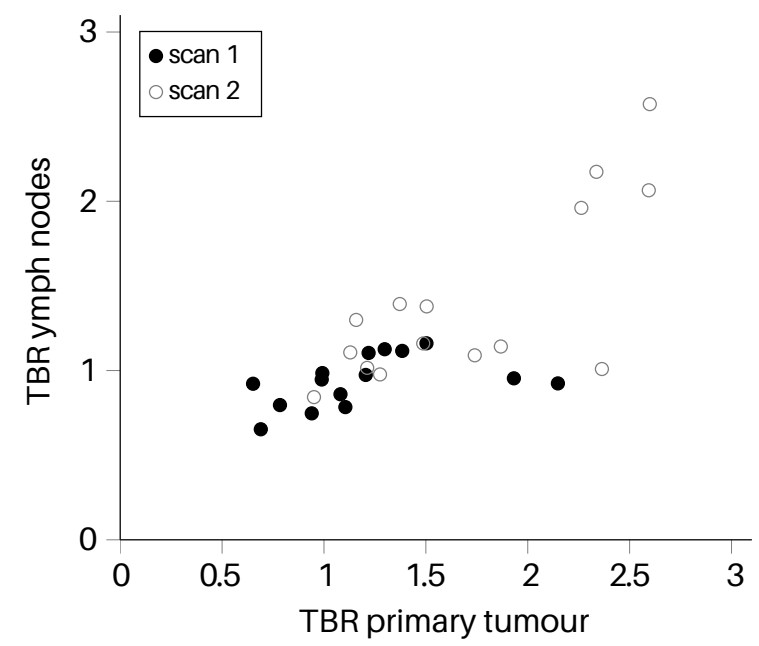

Figure S3.1. Correlation between the ${ }^{89} \mathrm{Zr}$-cetuximab tumour-to-background ratio in the primary tumour and the maximum TBR of the involved lymph nodes for scan $1(\mathrm{r}=0.446$, $\mathrm{p}=0.095)$ and scan $2(\mathrm{r}=0.764, \mathrm{p}<0.01)$. 
Table S3.1. Imaging characteristics for the ${ }^{89} \mathrm{Zr}$-cetuximab scans and FDG PET/CT scan for the primary tumour. Treatment characteristics and treatment outcome are also presented.

\begin{tabular}{|c|c|c|c|c|c|c|c|c|c|c|c|c|c|c|c|c|c|}
\hline \multirow[b]{2}{*}{ Patient } & \multicolumn{3}{|c|}{ FDG PET/CT } & \multicolumn{5}{|c|}{${ }^{89} \mathrm{Zr}$-cetuximab PET/CT scan 1} & \multicolumn{5}{|c|}{${ }^{89} \mathrm{Zr}$-cetuximab PET/CT scan 2} & \multirow{2}{*}{$\begin{array}{c}\text { Correlation } \\
{ }^{89} \mathrm{Zr} \text {-cetuximab } \\
\text { scans } \\
\mathrm{r}\end{array}$} & \multirow[t]{2}{*}{ Treatment \# arm } & \multirow[t]{2}{*}{$\begin{array}{l}\text { Locoregional } \\
\text { recurrence }\end{array}$} & \multirow[t]{2}{*}{ Metastasis } \\
\hline & $\mathrm{SUV}_{\text {peak }}$ & $\mathrm{SUV}_{\max }$ & $\mathrm{SUV}_{\text {mean }}$ & Day & $\mathrm{SUV}_{\text {peak }}$ & $\mathrm{SUV}_{\max }$ & $\mathrm{SUV}_{\text {mean }}$ & TBR & Day & $\mathrm{SUV}_{\text {peak }}$ & $\mathrm{SUV}_{\max }$ & $\mathrm{SUV}_{\text {mean }}$ & TBR & & & & \\
\hline 2 & 15.0 & 17.1 & 7.8 & 4 & 2.7 & 2.9 & 2.1 & 1.1 & 7 & 2.9 & 3.2 & 2.0 & 1.9 & 0.76 & Cetuximab & 0 & 0 \\
\hline 3 & 8.6 & 10.9 & 4.3 & 4 & 4.7 & 6.8 & 2.3 & 1.9 & 7 & 4.2 & 5.7 & 2.2 & 2.4 & 0.53 & Cetuximab & 0 & 0 \\
\hline 4 & 12.0 & 15.2 & 6.8 & 3 & 6.2 & 7.9 & 4.0 & 1.4 & 6 & 6.2 & 7.5 & 3.9 & 2.3 & 0.86 & Cisplatin & 0 & 0 \\
\hline 5 & 8.6 & 9.7 & 4.7 & 4 & 4.1 & 5.6 & 2.5 & 1.0 & 7 & 4.5 & 6.8 & 3.0 & 1.1 & 0.18 & Cisplatin & 0 & 0 \\
\hline 7 & 8.4 & 10.9 & 3.0 & 3 & 2.5 & 2.8 & 1.8 & 0.8 & 6 & 2.5 & 2.9 & 1.7 & 1.2 & 0.50 & Cisplatin & 0 & 0 \\
\hline 8 & 13.6 & 16.5 & 6.6 & 3 & 3.2 & 3.5 & 2.5 & 0.9 & 6 & 3.3 & 3.7 & 2.6 & 1.4 & 0.73 & Cetuximab & 1 & 0 \\
\hline 9 & 11.9 & 16.0 & 5.5 & 4 & 3.1 & 3.4 & 1.9 & 1.3 & 7 & 2.7 & 3.2 & 1.6 & 2.6 & 0.68 & Cetuximab & 1 & 0 \\
\hline 10 & 12.0 & 13.2 & 5.9 & 3 & 3.5 & 3.8 & 2.7 & 0.7 & 6 & 3.8 & 4.1 & 3.0 & 1.0 & 0.66 & Cetuximab & 0 & 0 \\
\hline 11 & 9.8 & 13.4 & 3.0 & 4 & 3.7 & 4.5 & 2.0 & 1.1 & 7 & 3.0 & 3.6 & 1.9 & 1.5 & 0.75 & Cetuximab & 0 & 0 \\
\hline 12 & 17.3 & 21.3 & 9.1 & 3 & 4.4 & 5.2 & 3.0 & 0.9 & 6 & 4.3 & 5.2 & 2.8 & 1.3 & 0.78 & Cetuximab & 1 & 1 \\
\hline 13 & 37.9 & 44.2 & 14.3 & NA & NA & NA & NA & NA & 7 & 5.7 & 6.5 & 3.4 & 2.6 & NA & Cisplatin & 0 & 1 \\
\hline 14 & 12.2 & 18.1 & 2.9 & 4 & 3.1 & 3.7 & 2.0 & 1.0 & 7 & 2.9 & 3.7 & 1.8 & 1.4 & 0.61 & Cisplatin & 0 & 0 \\
\hline 15 & 16.7 & 19.9 & 7.5 & 4 & 4.2 & 4.7 & 2.9 & 1.2 & 7 & 4.2 & 4.9 & 2.8 & 1.7 & 0.63 & Cisplatin & 0 & 0 \\
\hline 17 & 11.5 & 14.5 & 6.1 & 4 & 6.2 & 7.6 & 3.5 & 1.5 & 7 & 5.6 & 6.6 & 3.1 & 1.5 & 0.80 & Cisplatin & 0 & 0 \\
\hline
\end{tabular}

Abbreviations: SUV = standardized uptake value; TBR = tumour-to-background ratio; NA = not acquired. For patient 1 and 13 only the second scan was available for analysis . 
Table S3.2. Imaging characteristics for the ${ }^{89} \mathrm{Zr}$-cetuximab scans and FDG PET/CT scan for the lymph nodes.

\begin{tabular}{|c|c|c|c|c|c|c|c|c|c|c|}
\hline \multirow[b]{2}{*}{ Patient } & \multicolumn{2}{|c|}{ FDG PET/CT } & \multicolumn{4}{|c|}{${ }^{89} \mathrm{Zr}$-cetuximab PET/CT scan 1} & \multicolumn{4}{|c|}{${ }^{89} \mathrm{Zr}$-cetuximab PET/CT scan 2} \\
\hline & $\mathrm{SUV}_{\text {peak }}$ & $\mathrm{SUV}_{\max }$ & Day & $\mathrm{SUV}_{\text {peak }}$ & $\mathrm{SUV}_{\max }$ & TBR & Day & $\mathrm{SUV}_{\text {peak }}$ & $\mathrm{SUV}_{\max }$ & TBR \\
\hline 1 & 4.5 & 8.5 & NA & NA & NA & NA & 7 & 2.4 & 3.7 & 0.9 \\
\hline 2 & 4.0 & 7.3 & 4 & 2.1 & 2.3 & 0.9 & 7 & 1.8 & 1.9 & 1.1 \\
\hline 3 & 3.7 & 5.3 & 4 & 2.3 & 3.1 & 1.0 & 7 & 1.8 & 2.5 & 1.0 \\
\hline 4 & 18.9 & 21.4 & 3 & 5.0 & 5.5 & 1.1 & 6 & 5.4 & 5.7 & 2.0 \\
\hline 5 & 8.3 & 10.9 & 4 & 4.1 & 5.6 & 1.0 & 7 & 4.4 & 6.5 & 1.1 \\
\hline 6 & 18.6 & 20.9 & 3 & 5.2 & 6.4 & 1.1 & 6 & 5.0 & 6.7 & 2.2 \\
\hline 7 & 5.5 & 7.1 & 3 & 2.6 & 2.9 & 0.8 & 6 & 2.1 & 2.4 & 1.0 \\
\hline 8 & - & - & - & - & - & - & - & - & - & - \\
\hline 9 & 9.1 & 12.6 & 4 & 2.7 & 3.0 & 1.1 & 7 & 2.7 & 3.4 & 2.6 \\
\hline 10 & 6.2 & 7.7 & 3 & 3.3 & 3.9 & 0.7 & 6 & 3.3 & 3.8 & 0.8 \\
\hline 11 & 3.5 & 5.1 & 4 & 2.6 & 3.1 & 0.8 & 7 & 2.4 & 2.7 & 1.2 \\
\hline 12 & 7.9 & 11.4 & 3 & 3.5 & 4.2 & 0.8 & 6 & 3.3 & 4.0 & 1.0 \\
\hline 13 & 13.5 & 17.2 & NA & NA & NA & NA & 7 & 4.5 & 5.2 & 2.1 \\
\hline 14 & 2.4 & 3.2 & 4 & 3.0 & 3.4 & 1.0 & 7 & 2.9 & 3.4 & 1.4 \\
\hline 15 & 4.6 & 6.1 & 4 & 3.4 & 3.8 & 1.0 & 7 & 2.7 & 2.9 & 1.1 \\
\hline 16 & 10.3 & 14.1 & 4 & 4.0 & 4.5 & 0.9 & 7 & 3.7 & 4.3 & 1.3 \\
\hline 17 & 12.0 & 15.0 & 4 & 4.8 & 5.7 & 1.2 & 7 & 5.1 & 6.3 & 1.4 \\
\hline
\end{tabular}

Abbreviations: SUV = standardized uptake value; TBR = tumour-to-background ratio; NA = not acquired. For patient 1 and 13 only the second scan was available for analysis. Patient 8 did not have involved lymph nodes.

Table S3.3. Comparison of the mean uptake in the normal tissues (aorta and trapezius muscle), and in the primary tumour for the two ${ }^{89} \mathrm{Zr}$-cetuximab PET/CT scans. The SUV ${ }_{\text {peak }}$ of the tumour is compared to the uptake in the aorta, to calculate the tumour-to-background ratio (TBR), and compared to the uptake in the trapezius, to calculate the tumour-to-muscle ratio (TMR).

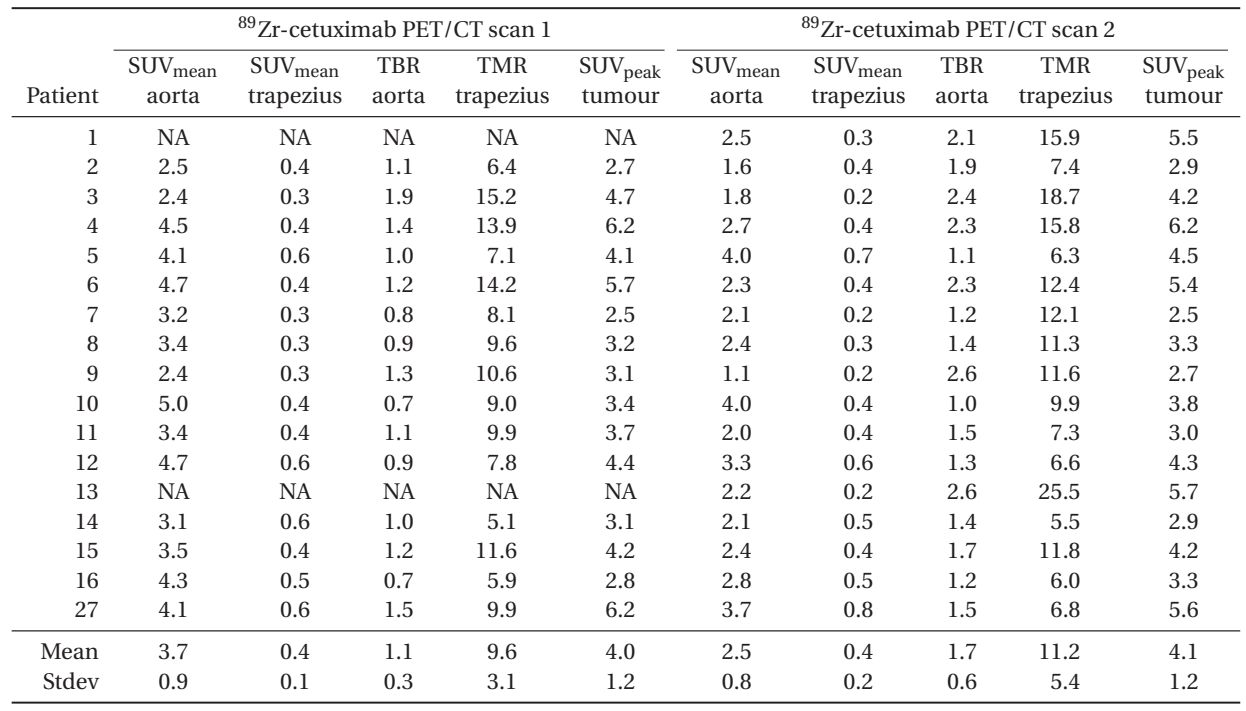

Abbreviations: SUV = standardized uptake value; TBR = tumour-to-background ratio; TMR = tumour-to-muscle ratio; NA = not acquired. For patient 1 and 13 only the second scan was available for analysis. 


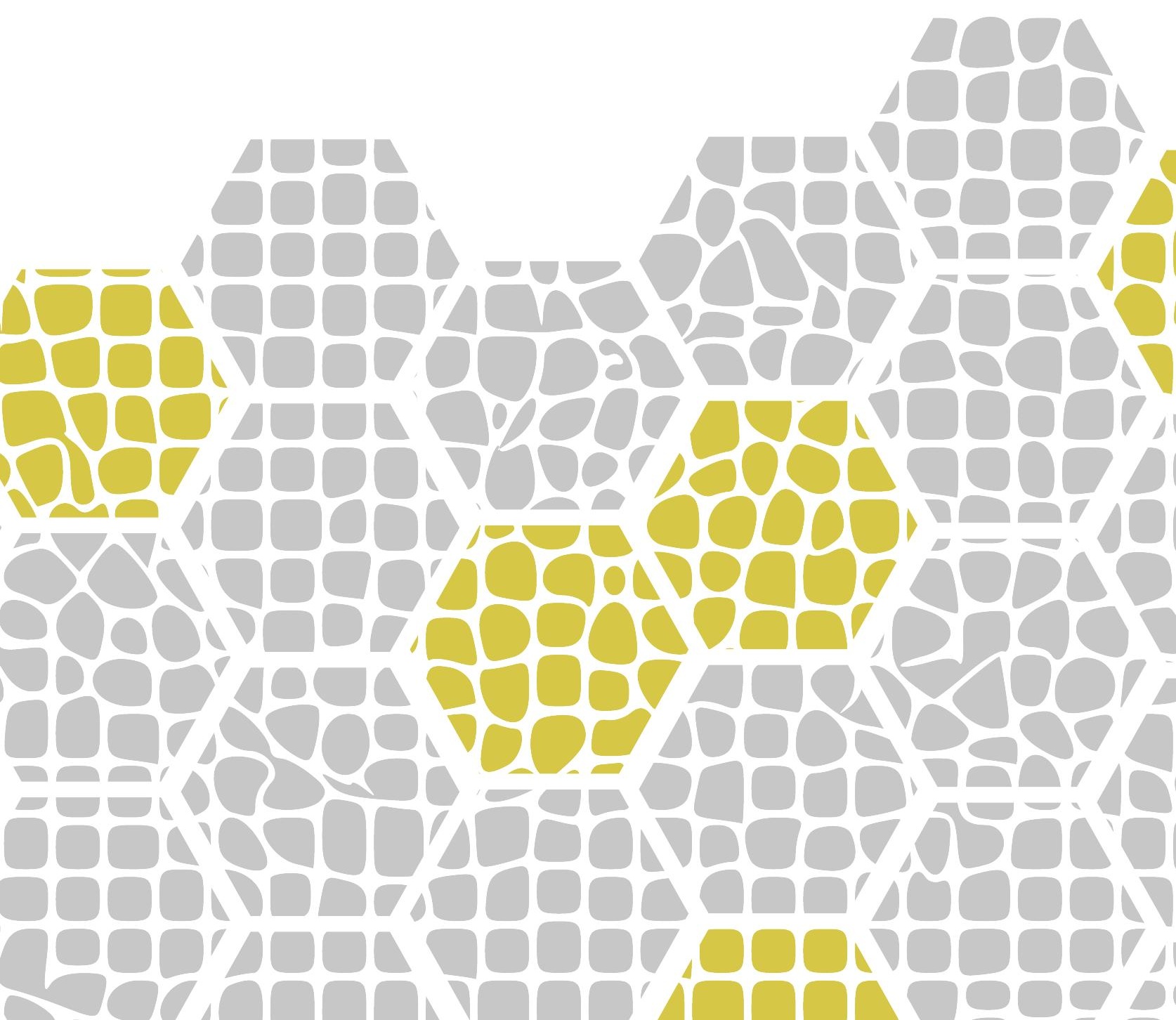




\section{Hypoxia and DCE-CT imaging for outcome prediction and treatment adaptation}

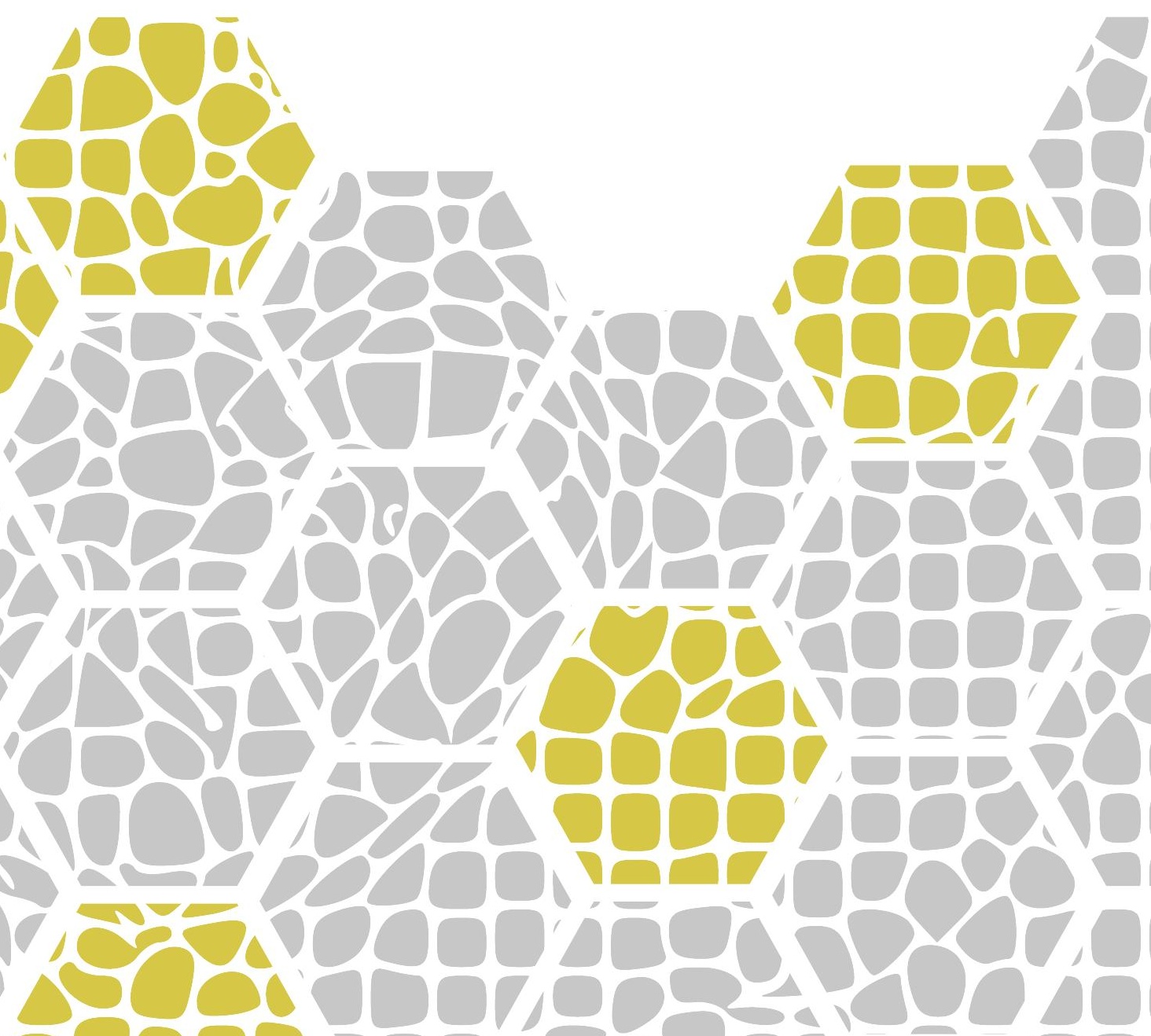




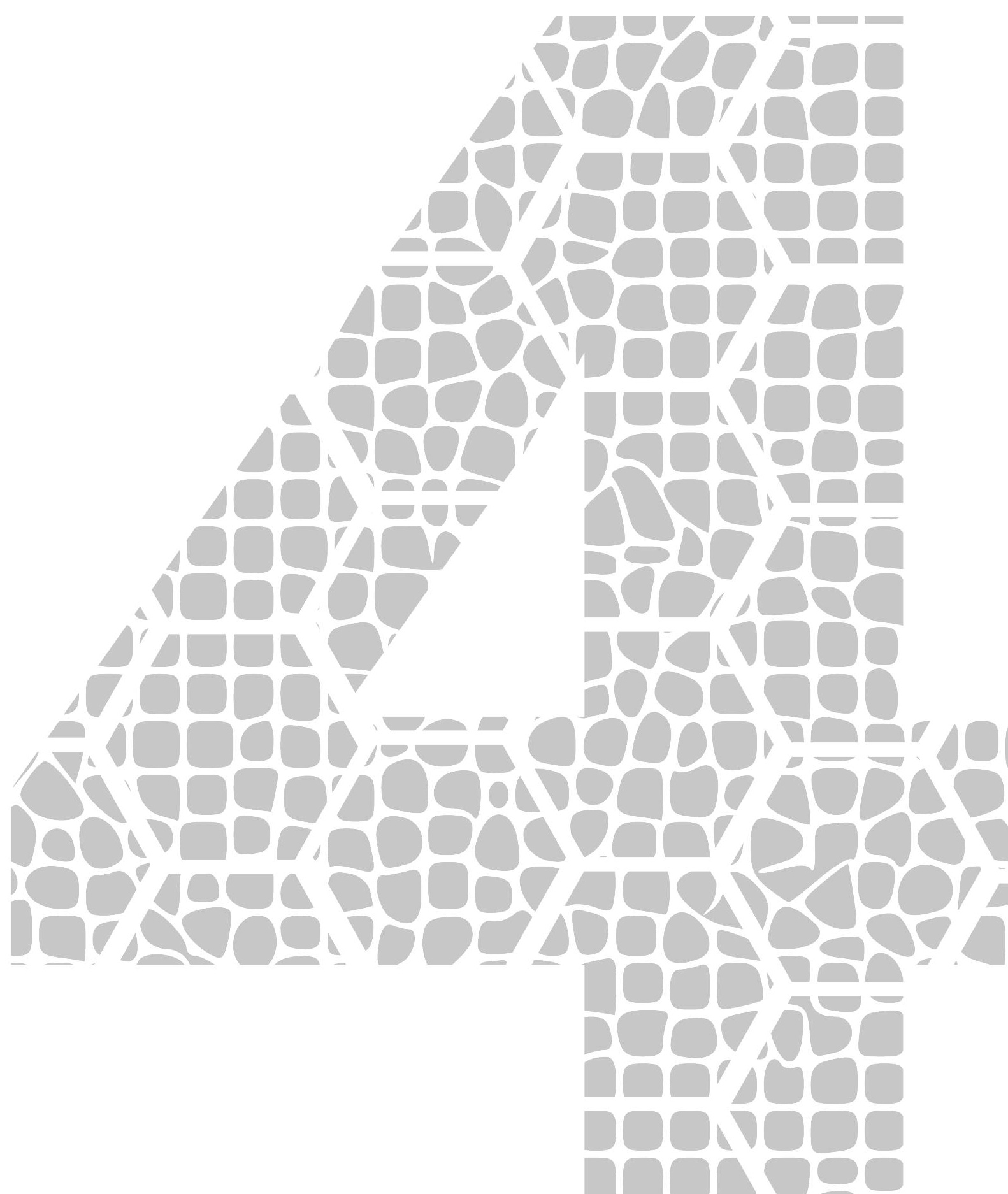




\section{Chapter 4}

\section{Prognostic and predictive value of hypoxia HX4 PET and DCE-CT imaging in non-small cell lung}

cancer: results of a prospective window-of-opportunity clinical trial with nitroglycerin

Aniek J.G. Even*, Bart Reymen", Catharina M.L. Zegers, Marco Das, Erik Vegt, Joachim E. Wilberger, Felix M. Mottaghy, Wouter van Elmpt, Dirk De Ruysscher, Philippe Lambin 


\begin{abstract}
Background Nitroglycerin, a vasodilating drug, is a potential candidate for targeting treatment resistant hypoxic tumour regions. It has been hypothesized that nitroglycerin reduces tumour hypoxia and improves perfusion, however, trials examining the added benefit of nitroglycerin to (chemo)radiotherapy have been inconclusive. A window-of-opportunity trial (NCT01210378) was designed to study the effect of nitroglycerin on hypoxia and tumour vasculature in non-small cell lung cancer (NSCLC) patients, and to study the potential of functional imaging for patient selection.
\end{abstract}

Material and methods Stage IB-IV NSCLC patients treated with curative intent using (chemo)radiotherapy, received a hypoxia HX4 PET/CT and a dynamic contrast-enhanced CT (DCE-CT) scan at baseline and after applying a transdermal nitroglycerin patch (Transiderm Nitro $5 \mathrm{mg}$ ). Patients were stratified based on hypoxia levels (TBR $>1.2$ ), median tumour blood flow (BF), and blood volume (BV). Differences in overall survival (OS), locoregional progression-free survival (LRPFS) and metastasis-free survival (MFS) were assessed using Kaplan Meier curves and log-rank tests. The survival of patients with a nitroglycerin related reduction in hypoxia was assessed separately.

Results Hypoxia HX4 PET/CT scans were acquired at baseline and after nitroglycerin administration for 32 and 25 patients, respectively; DCECT images were acquired for 22 and 13 patients at these time points. At baseline, significant differences between hypoxic and non-hypoxic tumours were found for OS (two-year OS: $47 \%$ vs $100 \%$; $=0.029$ ) and MFS (two-year MFS: $52 \%$ vs $100 \%$; $\mathrm{p}=0.045$ ), but not for LRPFS. DCE-CT derived parameters, at baseline or with nitroglycerin, could not be related to survival. Four patients showed a significant reduction in hypoxia in the primary tumour or lymph nodes after nitroglycerin administration. None of these four patients experienced locoregional failure.

Conclusion Tumour hypoxia assessed with HX4 PET is a prognostic biomarker for survival, in contrast to DCE-CT or FDG PET. Patients with hypoxic tumours at baseline had a worse OS compared to non-hypoxic tumours. The limited number of patients with a reduction in hypoxia, restricted the validation of predictive imaging biomarkers for nitroglycerin in this trial. 


\section{Introduction}

Tumour hypoxia is an independent negative prognostic factor in most solid cancers. The poorly regulated neovascularisation in tumours results in underdeveloped leaky vessels, with a highly irregular infrastructure, resulting in regions with low levels of oxygenation (i.e. hypoxia) [1]. These hypoxic regions are more resistant to both chemotherapy and radiotherapy [2]. The lack of oxygen reduces the efficiency of the DNA damage caused by radiotherapy, and the poor vasculature limits the distribution of chemotherapy [3]. Furthermore, the hypoxic microenvironment promotes a more aggressive, metastasis-prone phenotype [4,5]. All these factors combined, highlight the potential of hypoxia as a target for anticancer therapy.

Nitric oxide-donating drugs, such as nitroglycerin, are an example of drugs that have the potential to target hypoxia. Nitroglycerin is a commonly used vasodilator to treat cardiac diseases such as angina pectoris or heart failure. In addition to the wellknown cardiac effects, it also stimulates processes that may reduce tumour hypoxia. Nitroglycerin increases tumour blood flow, normalizes blood viscosity, stabilizes p53 and reduces hypoxia-inducible factor- $1 \alpha$ (HIF-1 $\alpha$ ) levels [6-8].

Several clinical trials have been conducted examining the added clinical benefit of nitroglycerin. Yasuda et al. showed a significant survival benefit for adding nitroglycerin to chemotherapy in a randomized phase II trial [9]. However, several other trials examining the combination of chemotherapy with nitroglycerin, including a large randomized multicentre phase II trial and a phase III trial, could not confirm these results [10-12]. For chemoradiotherapy combined with nitroglycerin, response and survival rates comparable to the standard arms of recent randomized trials on chemoradiation were found [13].

One major limitation of these clinical trials is the lack of patient selection; none of the patients were screened for tumour hypoxia before inclusion. Since nitroglycerin is hypothesized to mainly target hypoxia, trials without hypoxia based patient stratification might not show its full potential [14].

We designed a window-of-opportunity trial to test the possibility to preselect non-small cell lung cancer (NSCLC) patients that would most likely benefit from the addition of nitroglycerin to radiotherapy. We hypothesized that tumour hypoxia, acquired with HX4 PET/CT, and tumour vasculature, assessed with dynamic contrastenhanced CT (DCE-CT), are two important prognostic and possibly predictive factors for the benefit of nitroglycerin addition. In this study we analysed the prognostic value of hypoxia HX4 PET and DCE-CT imaging at baseline. So far, several studies have shown that nitroimidazole-based hypoxia PET tracers are prognostic for survival in NSCLC patients [15-18]. The prognostic value of the newer nitroimidazole hypoxia PET tracer HX4 [19-21] however, has not yet been assessed. Additionally, we assessed 
the effect of the nitroglycerin patch on hypoxia and tumour perfusion by comparing the baseline and nitroglycerin scans. The changes in imaging parameters between the two time points are explored as a potential predictive marker.

\section{Materials and Methods}

\section{Patient selection and treatment}

Stage IB-IV NSCLC patients, receiving radiotherapy with curative intent and with a WHO performance status $0-2$, were eligible for the prospective nitroglycerin trial (NCT01210378). Patients with recent severe cardiac disease, symptomatic hypotension, or poor renal function, were not eligible. The trial allowed inclusion of patients treated with radical radiotherapy, with or without standard chemotherapy (platinum doublet chemotherapy combined either with etoposide or vinorelbin). During the course of the trial, several standard radiotherapy regimens were used at Maastro clinic. Patients treated with stereotactic radiotherapy were treated with 8 fractions of 7.5 Gy delivered 3 days per week with a minimum interfraction interval of 48 hours. Patients treated without chemotherapy or with sequential chemoradiotherapy were treated using our individualized accelerated radiotherapy (INDAR) using twice daily fractions of $1.8 \mathrm{~Gy}$ per fraction up to an individual total tumour dose (TTD) limited by the dose constraints of the organs at risk (OAR). In concurrent chemoradiation, patients were treated with a different INDAR schedule consisting of 30 fractions of 1.5 Gy delivered twice daily, followed by an individually variable number of fractions of 2 Gy delivered once daily up to a TTD determined by the OAR constraints. Both INDAR schedules have been described in detail [22, 23]. The last patient included in the trial was treated concurrently with chemotherapy using 30 fractions of 2 Gy because the INDAR approach was abandoned shortly before his inclusion. Patient characteristics and treatment schedules are listed in Table 4.1.

In addition to the standard (chemo)radiotherapy, patients were asked to apply a transdermal nitroglycerin patch (Transiderm Nitro $5 \mathrm{mg}$, Novartis), throughout the course of radiotherapy, for 12 hours per day. These patches release $0.2 \mathrm{mg}$ nitroglycerin per hour and contain $25 \mathrm{mg} / 10 \mathrm{~cm}^{2}$. The trial was approved by the Medical Ethics Review Committee and all patients gave written informed consent.

\section{Hypoxia and DCE-CT image acquisition and image processing}

The imaging protocol consisted of two sets of HX4 PET/CT and DCE-CT images taken before the start of radiotherapy: one set without a nitroglycerin patch (baseline) and one with the patch (nitroglycerin scan). The second set of scans was scheduled at least 
Table 4.1. Patient characteristics of the 34 analysed patients.

\begin{tabular}{|c|c|c|}
\hline Patients & & 34 \\
\hline \multirow[t]{2}{*}{ Gender } & Male & 21 \\
\hline & Female & 13 \\
\hline Age (mean and range in years) & & $63(40-82)$ \\
\hline \multirow[t]{3}{*}{ GTV (median and range in $\mathrm{cm}^{3}$ ) } & Tumour & $23(1-477)$ \\
\hline & Nodes & $19(2-251)$ \\
\hline & Total & $64(6-497)$ \\
\hline \multirow[t]{2}{*}{ WHO-PS } & 0 & 6 \\
\hline & 1 & 28 \\
\hline \multirow[t]{5}{*}{ Treatment } & Radiotherapy & 4 \\
\hline & Stereotactic radiotherapy & 2 \\
\hline & Sequential chemoradiation & 1 \\
\hline & Concurrent chemoradiation & 25 \\
\hline & Radiotherapy with neoadjuvant chemotherapy & 2 \\
\hline \multirow{4}{*}{ Radiotherapy schedules } & 60 Gy/2Gy/QD & 1 \\
\hline & $60 \mathrm{~Gy} / 7.5 \mathrm{~Gy} / 3$ fractions per week & 2 \\
\hline & INDAR:1.8 Gy/BID & 8 \\
\hline & INDAR: 1.5 Gy/BID + 2Gy/QD & 23 \\
\hline \multirow[t]{4}{*}{ TNM (T) } & TX & 1 \\
\hline & $\mathrm{T} 2$ & 10 \\
\hline & T3 & 10 \\
\hline & $\mathrm{T} 4$ & 13 \\
\hline \multirow[t]{4}{*}{ TNM $(\mathrm{N})$} & No & 7 \\
\hline & N1 & 1 \\
\hline & N2 & 15 \\
\hline & N3 & 11 \\
\hline \multirow[t]{2}{*}{ TNM (M) } & M0 & 24 \\
\hline & M1 & 10 \\
\hline \multirow[t]{4}{*}{ Pathology } & Adenocarcinoma & 12 \\
\hline & Squamous cell carcinoma & 9 \\
\hline & Large-cell carcinoma & 6 \\
\hline & NSCLC NOS & 7 \\
\hline
\end{tabular}

Abbreviations: GTV = gross tumour volume, WHO-PS = World Health Organisation performance status, INDAR = individualized isotoxic accelerated radiotherapy, BID = twice-daily, NOS = not otherwise specified.

48 hours after baseline imaging, with the nitroglycerin patch applied at least one hour before the scan.

Hypoxia HX4 PET/CT scans were acquired according to a previously described protocol [24]. The scans were acquired 4 hours after injecting $413 \mathrm{MBq} \pm 80 \mathrm{MBq}$ HX4 PET tracer for the first scan or $444 \mathrm{MBq} \pm 27 \mathrm{MBq}$ HX4 PET tracer for the second scan. The scans were acquired on a Gemini TF64 PET/CT (Philips, Best, the 
Netherlands), in treatment position, on a flat table top, with arm and knee supports. The PET images were attenuation corrected, using a low-dose CT scan, and corrected for scatter and random coincidences. A BLOB-OS-TF algorithm (3 iterations and 33 subsets) was applied to reconstruct images of 4 by $4 \mathrm{~mm}$ with a slice thickness of $4 \mathrm{~mm}$. The measured uptake was corrected for body weight, injected dose, and decay to calculate the standardized uptake value (SUV). Tumour-to-background ratios (TBR) were calculated by dividing the SUV in the tumour by the average SUV in the aortic arch. The hypoxic volume (HV), in $\mathrm{cm}^{3}$, was determined by summing the volume of voxels in the tumour with a TBR $>1.2$. The fraction of HV (FHV) was calculated by dividing the $\mathrm{HV}$ by its respective tumour volume.

The DCE-CT imaging protocol was described previously [25]. In short, 33 consecutive $80 \mathrm{kVp}$ CT scans were acquired every 1.5 seconds after injecting 60 or $65 \mathrm{ml} \mathrm{(7} \mathrm{ml/s)} \mathrm{iodine-based} \mathrm{contrast} \mathrm{material} \mathrm{(Iopromide} \mathrm{300,} \mathrm{Bayer} \mathrm{Healthcare,}$ Berlin, Germany). The contrast material was followed by a saline chaser of 30 or $40 \mathrm{ml}$ $(7 \mathrm{ml} / \mathrm{s})$. Images were acquired in treatment position on a second or third generation dual source CT scanner (SOMATOM Definition Flash or SOMATOM Force, Siemens Healthcare, Erlangen, Germany), and reconstructed using a B20f filter on $0.8 \times 0.8 \mathrm{~mm}$ voxels with a slice thickness of $5 \mathrm{~mm}$ and slice increment of $3 \mathrm{~mm}$. Patients were asked to perform expiration breath hold and continue shallow breathing if breath hold could not be maintained. The Siemens noise reduction was applied. To compensate for any movement, all CT scans were registered to the first CT frame [26]. The aortic arch was delineated to calculate the aortic input function. A deconvolution algorithm was used for kinetic modelling to create parametric maps of blood volume (BV), in ml/100 ml, and blood flow (BF), in $\mathrm{ml} / 100 \mathrm{ml} / \mathrm{min}$ (syngo MMWP, version VE40A, Siemens). The mean tumour BF and BV were calculated for each patient. DCE-CT scans with incorrect timing, either acquired too early or too late after contrast administration, missing a part of the uptake curve in the tumour or aorta, were omitted.

\section{Radiotherapy planning and tumour delineation}

The majority of patients received a 4D FDG PET/CT on a Siemens Biograph 40 PET/CT scanner (Siemens Healthcare, Erlangen, Germany) one hour after injection of FDG, for radiotherapy treatment planning purposes. The PET scan was attenuation corrected using the midventilation phase of the CT image and corrected for scatter and random coincidences. For two patients, treatment planning was performed on a dedicated CT scan (Sensation Open, Siemens Healthcare, Erlangen, Germany). Delineation of the primary gross tumour volume $\left(\mathrm{GTV}_{\text {prim }}\right)$ and involved lymph nodes $\left(G_{\mathrm{ln}}\right)$ was performed by an experienced radiation oncologist on the fused PET and midventilation CT scan or the dedicated CT scan. 


\section{Coregistration of the images}

The CT of the hypoxia PET/CT and the first CT scan of the DCE-CT were registered to the CT of the FDG PET/CT or the dedicated planning CT using a two-step process; the scans were aligned using a non-deformable registration and consecutively deformed using a non-rigid registration (Elastix $[27,28]$ ). The deformation field of the hypoxia CT was applied on the HX4 PET, and the deformation field of the DCE-CT was applied on the BV and BF maps.

\section{Survival analysis and response to nitroglycerin}

Follow-up for all patients was performed according to the regional standard follow-up protocol. This includes a CT scan at three months after radiotherapy, repeated yearly afterwards and whenever clinically indicated. The overall survival (OS), locoregional progression-free survival (LRPFS) and metastasis-free survival (MFS) were determined for all patients. The overall survival was defined as the time between the pathology diagnosis and death from any cause. LRPFS and MFS were defined from the time of pathology until the first sign of progression on imaging, which was either a recurrence in the primary tumour or regional lymph nodes, or a distant metastasis.

Kaplan Meier curves were used to analyse OS, LRPFS and MFS for the HX4 PET and DCE-CT scans at baseline and after applying the nitroglycerin patch. For the survival analysis, patients were split into hypoxic tumours (TBR > 1.2) and nonhypoxic tumours (TBR $<1.2$ ). For the DCE-CT scans, the median BF and BV were used for patient stratification. In addition, baseline median GTV size and median FDG $\mathrm{SUV}_{\text {max }}$ and $S U V_{\text {mean }}$ were tested for their prognostic value.

The response to nitroglycerin was assessed by comparing the baseline and nitroglycerin scans for hypoxia PET and DCE-CT. Changes in hypoxia were marked as significant, if they exceeded the previously determined coefficient of repeatability (CR). The absolute CR was 0.30 for the TBR and $14.9 \%$ for the FHV with a threshold of 1.2 times the background [29]. The survival of patients with a significant reduction in hypoxia were analysed separately.

\section{Statistical analysis}

The data were analysed using R: A Language and Environment for Statistical Computing (v3.3.2, Vienna, Austria; survival package v2.38). For the imaging parameters, the median and interquartile range (IQR) of the group of patients are provided. The survival statistics are presented as the median with the $95 \%$ confidence interval (CI). Survival differences between the several groups were statistically tested using a log-rank test. A p-value $<0.05$ was considered statistically significant. 


\section{Results}

In total 42 patients were included in the nitroglycerin clinical trial between December 2011 and June 2016. Of those patients, 34 patients (21 male, 13 female; mean age 63 years \pm 11 years) had at least a baseline HX4 PET/CT or baseline DCE-CT scan and were included in this study. A primary tumour was present in 33 out of 34 patients; involved lymph nodes were detected in 27 out of 34 patients. The patient details of the analysed patients are given in Table 4.1. The majority of patients ( $n=17$ ) were treated with concurrent chemoradiotherapy of 69 Gy in 42 fractions. Two patients were treated with stereotactic radiotherapy (60 Gy in 8 fractions of $7.5 \mathrm{~Gy})$. For the other patients $(\mathrm{n}=15)$, the prescribed radiotherapy dose ranged from 53-75 Gy in 30-41 fractions, and patients received either concurrent chemotherapy ( $n=8)$, neoadjuvant chemotherapy $(n=2)$, sequential chemotherapy $(n=1)$ or no chemotherapy $(n=4)$. At baseline, of the 34 patients analysed in this study, 32 patients received a FDG PET/CT, 32 patients a HX4 PET/CT and 22 patients received a DCECT scan. After applying the nitroglycerin patch, HX4 PET/CT scans were acquired for 25 patients and DCE-CT scans for 13 patients. Nitroglycerin scans were only acquired for patients with a baseline scan. In Figure 4.1, HX4 PET/CT scans, and BF and BV DCE-CT maps at baseline and after applying the nitroglycerin scans of two example patients are shown.

For all patients with a baseline scan, the median HX4 TBR was 1.4 (IQR: 1.2-1.7), the median HV $2.9 \mathrm{~cm}^{3}$ (IQR: $0.1-12.1 \mathrm{~cm}^{3}$ ), BF $63.5 \mathrm{ml} / 100 \mathrm{ml} / \mathrm{min}$ (IQR: 52.0-75.3 ml/100 ml/min), BV $7.1 \mathrm{ml} / 100 \mathrm{ml}$ (IQR: 5.5-9.7 ml/100 ml), FDG SUV $\max$ 10.9 (IQR: 7.2-14.5), and the median FDG SUV mean 4.2 (IQR: 2.7-5.7). Only weak correlations were observed between baseline hypoxia features and GTV size, for TBR $(\mathrm{r}=0.49)$ and FHV $(\mathrm{r}=0.27)$.

For the patients with both scans, the changes in imaging characteristics in the primary tumour after applying the nitroglycerin patch are shown in Figure 4.2. Both increases and decreases were observed on a patient level, for all imaging features. For the 24 patients with HX4 PET imaging available and a primary tumour, the median TBR remained unchanged between baseline (1.4; IQR: 1.2-1.8) and the nitroglycerin scan (1.4; IQR: 1.3-1.8). For the other hypoxia features only minor differences were observed, see Figure 4.2. For the 13 patients with both DCE-CT scans and a primary tumour, the median BF decreased from $63.6 \mathrm{ml} / 100 \mathrm{ml} / \mathrm{min}$ (IQR: $52.0-81.2 \mathrm{ml} / 100 \mathrm{ml} / \mathrm{min}$ ) to $53.8 \mathrm{ml} / 100 \mathrm{ml} / \mathrm{min}$ (IQR: $44.8-78.4 \mathrm{ml} / 100 \mathrm{ml} / \mathrm{min}$ ), and the median BV decreased from $7.5 \mathrm{ml} / 100 \mathrm{ml}$ (IQR: $5.8-9.4 \mathrm{ml} / 100 \mathrm{ml}$ ) to $7.2 \mathrm{ml} / 100 \mathrm{ml}$ (IQR: 6.9-8.6 ml/100 ml), see Figure 4.2.

The median follow-up time was 39 months, with follow-up ranging between 11 and 64 months for the patients alive. The two-year overall survival for the group of 

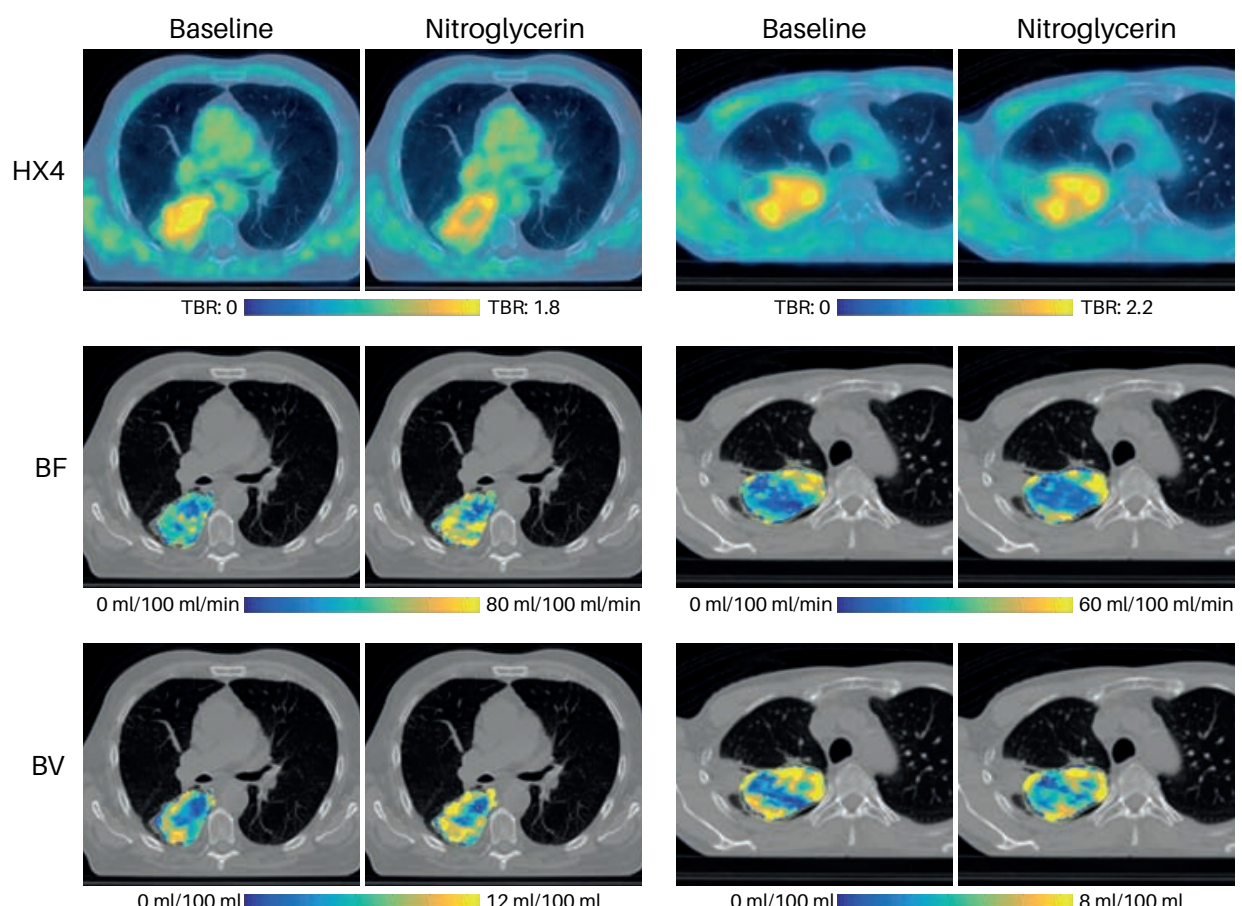

Figure 4.1. HX4 PET/CT scan, and blood flow (BF) and blood volume (BV) DCE-CT maps of two example patients, at baseline and after applying a nitroglycerin patch. Both patients had a hypoxic tumour at baseline. In the patient on the left, the hypoxic volume decreases after administration of nitroglycerin (from $70 \mathrm{~cm}^{3}$ to $64 \mathrm{~cm}^{3}$ ), while the mean BF (from 37 $\mathrm{ml} / 100 \mathrm{ml} / \mathrm{min}$ to $54 \mathrm{ml} / 100 \mathrm{ml} / \mathrm{min}$ ) and mean BV (from $5.8 \mathrm{ml} / 100 \mathrm{ml}$ to $8.6 \mathrm{ml} / 100 \mathrm{ml}$ ) increase. In the patient on the right, hypoxia and tumour perfusion remain almost unchanged, with a HV of $61 \mathrm{~cm}^{3}$ and $59 \mathrm{~cm}^{3}$, a BF of $32 \mathrm{ml} / 100 \mathrm{ml} / \mathrm{min}$ and $31 \mathrm{ml} / 100 \mathrm{ml} / \mathrm{min}$, and a BV of $5.8 \mathrm{ml} / 100 \mathrm{ml}$ and $5.7 \mathrm{ml} / 100 \mathrm{ml}$, at baseline and after nitroglycerin administration respectively.

34 patients described in this paper was $58 \%$ (95\% CI: 44-78 \%); the median overall survival was not yet reached at the time of analysis. Thirteen of the 34 patients (38\%) developed metastatic disease over time. The two-year metastasis-free survival was 63 \% (95 \% CI: 48-83\%). Eight patients (24\%) presented with a locoregional recurrence. Recurrences inside the original PTV occurred in five patients (15\%). The two-year locoregional relapse free survival was 75 \% (95 \% CI: 60-93 \%).

The Kaplan Meier curves, assessing the prognostic value of baseline imaging, are presented in Figure 4.3, for hypoxia PET imaging, and in Supplementary Figure S4.1, for DCE-CT imaging. A significant difference in OS ( $p=0.029)$ was observed between patients with hypoxic tumours (two-year OS $47 \%$; $95 \%$ CI: 31-72\%) and non-hypoxic tumours (two-year OS 100 \%; 95 \% CI: 100-100 \%). Patients with hypoxic tumours also suffered from a worse MFS ( $p=0.045$ ), with a two-year MFS of 52 \% (95 \% CI: 34-79\%), 

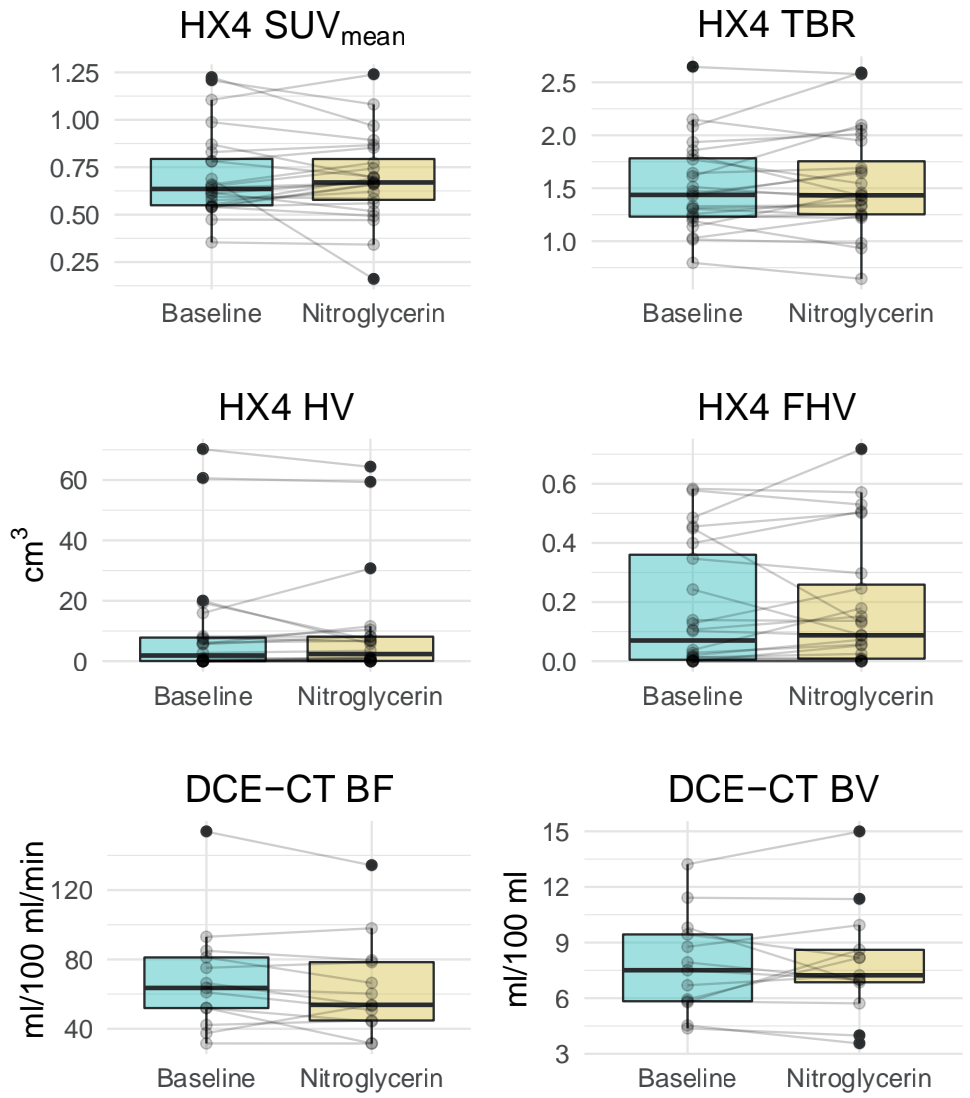

Figure 4.2. Boxplots of HX4 PET and DCE-CT imaging characteristics for the primary tumour for the patients with both a baseline and nitroglycerin scan. For the HX4 PET, the mean uptake (SUV mean), tumour-to-background ratio (TBR), hypoxic volume (HV), and fraction of HV (FHV) are given. For the DCE-CT images, the average blood flow (BF) and blood volume (BV) are shown. The observations of a single patient are connected with a line. The HX4 PET imaging features are shown for 24 patients; the DCE-CT features were available for 13 patients. 

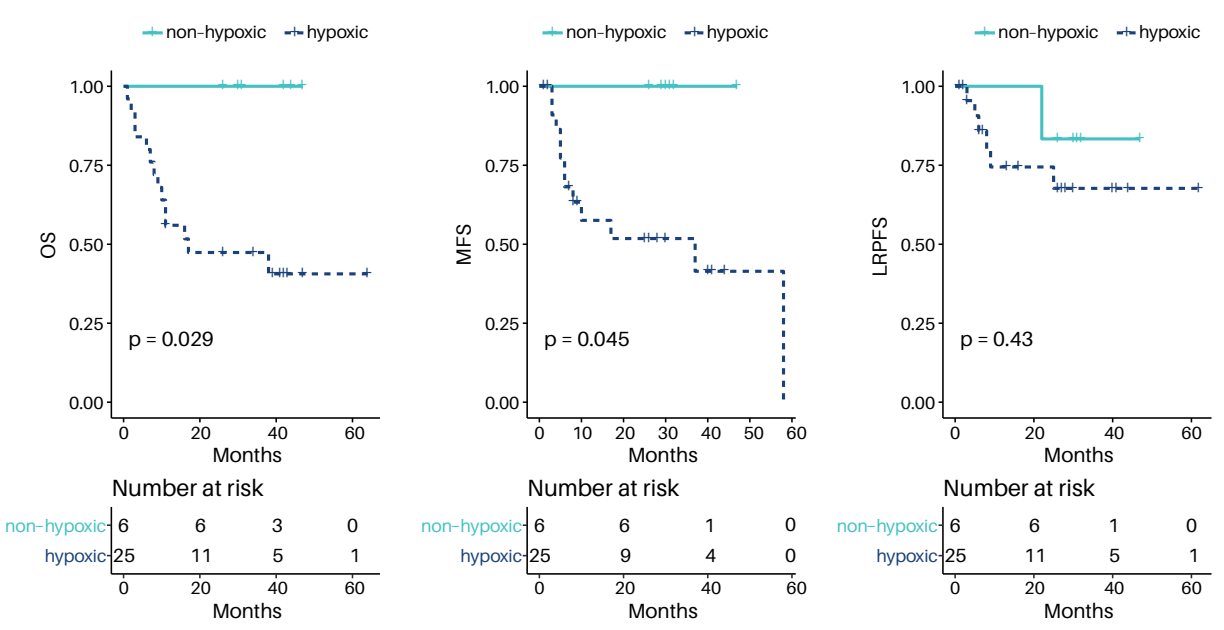

Figure 4.3. Prognostic value of baseline HX4 PET imaging of the primary tumour, with from left to right the overall survival (OS), metastasis-free survival (MFS), and locoregional progressionfree survival (LRPFS). In total 32 patients received a baseline HX4 PET/CT. The 31 patients with a primary tumour are displayed in the figure.

compared to 100 \% (95 \% CI: 100-100 \%) for non-hypoxic tumours. LRPFS was not significantly different between hypoxic and non-hypoxic tumours $(p=0.23)$. For the baseline DCE-CT scans, no significant differences were observed between patients with blood flow or blood volume above or below the median, for OS (BF: $p=0.7$; BV: $\mathrm{p}=0.45$ ), MFS (BF: $\mathrm{p}=0.58 ; \mathrm{BV}: \mathrm{p}=0.15$ ) and LRPFS (BF: $\mathrm{p}=0.49 ; \mathrm{BV}: \mathrm{p}=0.48$ ). The cumulative GTV of the primary tumour and involved lymph nodes (OS: $\mathrm{p}=0.94$; MFS: $\mathrm{p}=0.35$; LRPFS: $\mathrm{p}=0.56$ ), baseline FDG SUV $\max$ (OS: $\mathrm{p}=0.72$; MFS: $\mathrm{p}=0.94$; LRPFS: $\mathrm{p}=0.48$ ), and baseline FDG SUV mean (OS: $\mathrm{p}=0.62$; MFS: $\mathrm{p}=0.31$; LRPFS: $\mathrm{p}=0.74$ ) were also not prognostic in this patient cohort.

Kaplan Meier curves based on the scans with the nitroglycerin patch are shown in Figure 4.4 for hypoxia PET imaging, and in Supplementary Figure S4.2 for the DCE-CT imaging features. For the 24 patients with a nitroglycerin scan and primary tumour, no significant differences were found between patients with hypoxic and non-hypoxic tumours for OS ( $p=0.14)$, MFS ( $p=0.19$ ), or LRFS ( $p=0.99$ ). No survival difference was found for BF or BV for the 13 patients with a nitroglycerin DCE-CT scan.

A reduction in hypoxia, after applying the nitroglycerin patch, exceeding the coefficient of repeatability, was found in one $\mathrm{GTV}_{\text {prim }}$ based on the TBR threshold and two primary GTVs based on the FHV threshold. In total, two patients had a significant decrease in hypoxia in the primary tumour. In the first patient, the FHV decreased from 0.45 to 0.13 ; in the second patient, the FHV decreased from 0.24 to 0.09 . None of the primary tumours changed from hypoxic into non-hypoxic after the nitroglycerin 

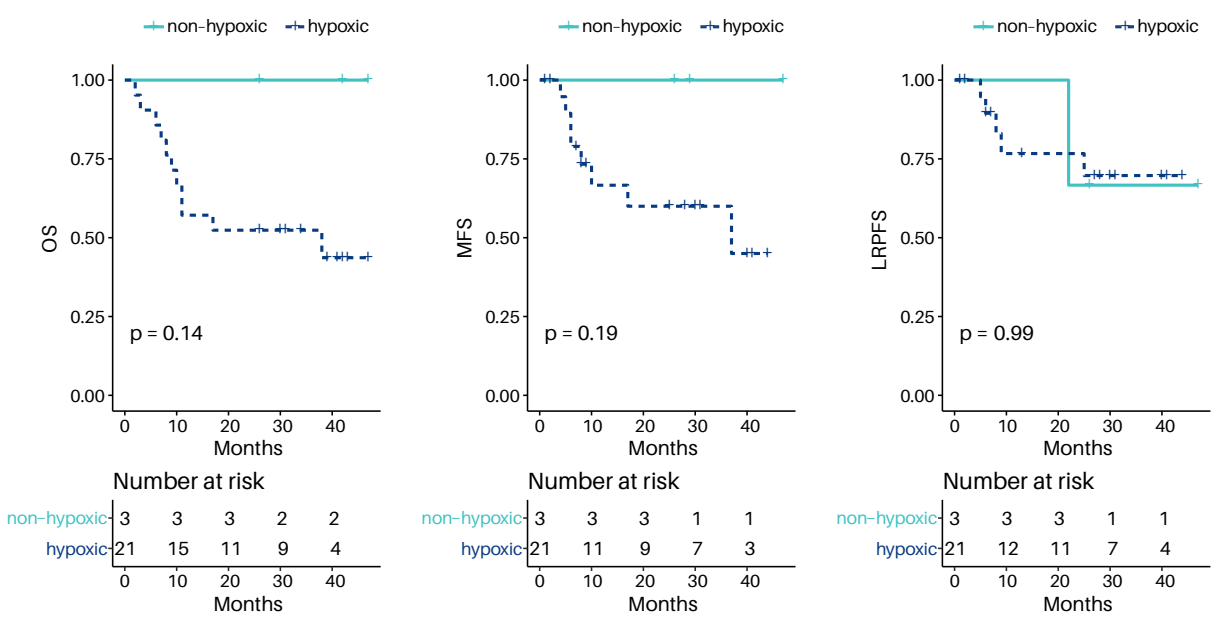

Figure 4.4. Prognostic value of HX4 PET imaging of the primary tumour after administration of nitroglycerin, with from left to right the overall survival (OS), metastasis-free survival (MFS), and locoregional progression-free survival (LRPFS). In total 25 patients received a nitroglycerin HX4 PET/CT. The 24 patients with a primary tumour are displayed in the figure.

administration, based on the TBR 1.2 threshold. Of the two patients with a significant decrease, the first patient developed metastases in the adrenal glands, paraaortic lymph nodes and longs and had an OS of 6 months. The second patient did not have any recurrences and was still alive after a follow-up period of 28 months.

In the first patient, in addition to a hypoxia decrease in the $\mathrm{GTV}_{\text {prim, }}$, also a significant reduction of hypoxia in the lymph nodes was observed. Two other patients showed a hypoxia reduction in the lymph nodes only. For one patient, a significant reduction of the FHV (from 0.20 to 0.03 ) was found. In the other patient, the lymph nodes were hypoxic at baseline $(\mathrm{TBR}=1.3)$ and non-hypoxic after nitroglycerin administration ( $\mathrm{TBR}=1.1$ ). These last two patients were alive at the last follow-up, with a follow-up of 39 and 42 months respectively. No locoregional progression was found in the two patients. The last patient developed metastatic disease after 17 months.

\section{Discussion}

Despite several hypoxia reducing properties that have been attributed to the vasodilating drug nitroglycerin, the added value of nitroglycerin in oncology is still inconclusive. The only other trial, to our knowledge, that has studied the benefit of adding nitroglycerin to (chemo)radiotherapy in NSCLC patients found an average response rate and acceptable toxicity levels [13]. For the 34 patients studied in this manuscript, a two-year OS of $58 \%$ (95\% CI: 44-78\%) was found. The survival of 
this heterogeneous stage IB-IV patient group was comparable to standard treatment in stage III NSCLC patients [30].

Mixed responses to nitroglycerin were observed between patients, which both increases and decreases in hypoxic levels, blood flow, and blood volume. Highlighting the need for patient stratification and selection. We hypothesized that nitroglycerininduced differences in hypoxia levels, as measured on HX4 PET/CT scans, could aid selection of patients for nitroglycerin treatment. However, due to the limited number of patients and the heterogeneous patient group, we could not confirm this hypothesis. Of all 42 patients included in the clinical trial, a baseline hypoxia PET scan was acquired for 32 patients, and only 25 of those patients received a nitroglycerin hypoxia PET. Most of the scans were not performed due to logistic reasons, or patients were not willing or able to undergo the scans. Of the 32 patients scanned at baseline, 25 patients had a hypoxic tumour at baseline and only two patients showed a significant reduction in hypoxia in the primary tumour and two patients in the lymph nodes. For one patient, the lymph nodes were hypoxic at baseline and non-hypoxic after nitroglycerin administration. Although none of these patients experienced locoregional failure, the patient number was insufficient for any statistical analysis on survival differences. Patient stratification based on imaging characteristics can be a powerful method for treatment personalization, yet it should be recognized that it rapidly decreases the number of patients available for analysis [31].

The threshold used to divide the patients in nitroglycerin responders and nonresponders based on their hypoxia status may have been too strict for this study and may have limited the number of detectable responders. The HX4 PET scans that were used to calculate the coefficients of repeatability were acquired shortly after injection of the PET tracer (90 minutes postinjection) and with short acquisition times (3 minutes per bed position) [29]. These scans will have suboptimal contrast to noise ratios compared to the scans used in this study (acquired at 4 hours postinjection, with 20 minutes per bed position) [24]. The expected lower noise levels in this study will arguably yield a higher reproducibility, thus smaller changes in hypoxia levels could be ascribed to the nitroglycerin administration.

This study showed the prognostic value of baseline HX4 PET/CT imaging for OS in NSCLC patients. Other nitroimidazole based PET tracers, FMISO, FAZA and FETNIM [15-18], already showed to be prognostic in NSCLC, however, no survival data was available until now for HX4 PET imaging. In this study we used a threshold of TBR > 1.2 to distinguish between hypoxic and non-hypoxic tumours. However, a formally established threshold is missing and several thresholds have been proposed for nitroimidazole-based PET tracers [24]. The Kaplan Meier curves for another commonly applied threshold, TBR > 1.4, are shown in Supplementary Figure S4.3 and Supplementary Figure S4.4. These thresholds should be further validated in a larger 
patient cohort.

For tumour size, FDG PET features, and DCE-CT imaging parameters, no survival differences were observed. This is in contradiction to other research showing the prognostic value of tumour volume [32,33] and FDG PET [34]. It has to be noted though, that the studied group of patients is possibly too small and too heterogeneous to show survival differences based on these features. NSCLC patients stage IB-IV were included, receiving a wide range of (combined) treatments. Multiple factors could have influenced survival, but further subgroup analysis is restricted due to the limited number of patients. DCE-CT scans were, similar to the HX4 PET scans, missing due to logistic reasons, or patients refused additional scans, or the timing of the scans was incorrect.

A final limitation of this study is the averaging of all imaging features over the whole tumour. It is well established that the tumour vasculature is highly irregular, and differences in tumour perfusion and hypoxia levels can be very local and heterogeneous $[1,35]$. By averaging the imaging features over a large region, subtle regional nitroglycerin effects might be unappreciated. It is possible that the distribution or redistribution of the blood flow, and not the average, may be more relevant for reducing hypoxia and improving chemotherapy accessibility [1]. Subregional tumour analysis could yield valuable information on local differences, however, the small patients group limits this more advanced image analysis. In a larger study cohort, more advanced imaging analysis methods may provide more insight [36, 37].

In conclusion, we presented the results of a window-of-opportunity trial on nitroglycerin. Patients with a hypoxic tumour at baseline, as determined on HX4 PET/CT, had a worse overall survival, compared to non-hypoxic tumours. In contrast, DCE-CT and FDG PET imaging features, and tumour volume could not be linked to patient prognosis. Confirmation of the prognostic value of HX4 PET is needed in a larger patient cohort.

\section{Acknowledgements}

Authors acknowledge financial support from ERC advanced grant (ERC-ADG-2015, $\mathrm{n}^{\circ} 694812$ - Hypoximmuno) and the QuIC-ConCePT project, which is partly funded by EFPI A companies and the Innovative Medicine Initiative Joint Undertaking (IMI JU) under Grant Agreement No. 115151. This research is also supported by the Dutch technology Foundation STW (grant $n^{\circ} 10696$ DuCAT \& $n^{\circ}$ P14-19 Radiomics STRaTegy), which is the applied science division of NWO, and the Technology Programme of the Ministry of Economic Affairs. Authors also acknowledge financial support from the EU 7th framework program (ARTFORCE - $\mathrm{n}^{\circ}$ 257144), SME Phase 
2 (RAIL - n' 673780), EUROSTARS (SeDI, CloudAtlas, DART), the European Program H2020-2015-17 (ImmunoSABR - n 733008 and PREDICT - ITN - n 766276), Interreg V-A Euregio Meuse-Rhine ("Euradiomics"), Kankeronderzoekfonds Limburg from the Health Foundation Limburg, Alpe d'HuZes-KWF (DESIGN), the ZuyderlandMAASTRO grant and the Dutch Cancer Society. 


\section{References}

1. Jain, R. K. Normalization of tumor vasculature: an emerging concept in antiangiogenic therapy. Science 307, 58-62 (2005).

2. Brown, J. M. \& Wilson, W. R. Exploiting tumour hypoxia in cancer treatment. Nat Rev Cancer 4, 437-47 (2004).

3. Wilson, W. R. \& Hay, M. P. Targeting hypoxia in cancer therapy. Nat Rev Cancer 11, 393-410 (2011).

4. Wouters, B. G., Koritzinsky, M., Chiu, R. K., Theys, J., Buijsen, J. \& Lambin, P. Modulation of cell death in the tumor microenvironment. Semin Radiat Oncol 13, 31-41 (2003).

5. Wouters, B. G., van den Beucken, T., Magagnin, M. G., Lambin, P. \& Koumenis, C. Targeting hypoxia tolerance in cancer. Drug Resist Updat 7, 25-40 (2004).

6. Jordan, B. F., Misson, P., Demeure, R., Baudelet, C., Beghein, N. \& Gallez, B. Changes in tumor oxygenation/perfusion induced by the no donor, isosorbide dinitrate, in comparison with carbogen: monitoring by EPR and MRI. Int J Radiat Oncol Biol Phys 48, 565-70 (2000).

7. Hagen, T., Taylor, C. T., Lam, F. \& Moncada, S. Redistribution of intracellular oxygen in hypoxia by nitric oxide: effect on HIFlalpha. Science 302, 1975-8 (2003).

8. Thomas, D. D., Espey, M. G., Ridnour, L. A., et al. Hypoxic inducible factor lalpha, extracellular signal-regulated kinase, and p53 are regulated by distinct threshold concentrations of nitric oxide. Proc Natl Acad Sci U S A 101, 8894-9 (2004).

9. Yasuda, H., Yamaya, M., Nakayama, K., et al. Randomized phase II trial comparing nitroglycerin plus vinorelbine and cisplatin with vinorelbine and cisplatin alone in previously untreated stage IIIB/IV non-small-cell lung cancer. J Clin Oncol 24, 688-94 (2006).

10. Reinmuth, N., Meyer, A., Hartwigsen, D., et al. Randomized, double-blind phase II study to compare nitroglycerin plus oral vinorelbine plus cisplatin with oral vinorelbine plus cisplatin alone in patients with stage IIIB/IV non-small cell lung cancer (NSCLC). Lung Cancer 83, 363-8 (2014).

11. Davidson, A., Veillard, A. S., Tognela, A., et al. A phase III randomized trial of adding topical nitroglycerin to first-line chemotherapy for advanced nonsmallcell lung cancer: the Australasian lung cancer trials group NITRO trial. Ann Oncol 26, 2280-6 (2015). 
12. Dingemans, A. M. C., Groen, H. J. M., Herder, G. J. M., et al. A randomized phase II study comparing paclitaxel-carboplatin-bevacizumab with or without nitroglycerin patches in patients with stage IV nonsquamous nonsmall-cell lung cancer: NVALT12 (NCT01171170). Ann Oncol 26, 2286-93 (2015).

13. Arrieta, O., Blake, M., de la Mata-Moya, M. D., et al. Phase II study. Concurrent chemotherapy and radiotherapy with nitroglycerin in locally advanced nonsmall cell lung cancer. Radiother Oncol 111, 311-5 (2014).

14. Dewhirst, M. W. \& Birer, S. R. Oxygen-enhanced MRI is a major advance in tumor hypoxia imaging. Cancer Res 76, 769-72 (2016).

15. Eschmann, S. M., Paulsen, F., Reimold, M., et al. Prognostic impact of hypoxia imaging with ${ }^{18} \mathrm{~F}$-misonidazole PET in non-small cell lung cancer and head and neck cancer before radiotherapy. J Nucl Med 46, 253-60 (2005).

16. Li, L., Hu, M., Zhu, H., Zhao, W., Yang, G. \& Yu, J. Comparison of $\left[{ }^{18} \mathrm{~F}\right]-$ fluoroerythronitroimidazole and $\left[{ }^{18} \mathrm{~F}\right]$-fluorodeoxyglucose positron emission tomography and prognostic value in locally advanced non-small-cell lung cancer. Clin Lung Cancer 11, 335-40 (2010).

17. Kinoshita, T., Fujii, H., Hayashi, Y., Kamiyama, I., Ohtsuka, T. \& Asamura, H. Prognostic significance of hypoxic PET using ${ }^{18} \mathrm{~F}$-FAZA and ${ }^{64} \mathrm{Cu}$-ATSM in nonsmall-cell lung cancer. Lung Cancer 91, 56-66 (2016).

18. Vera, P., Thureau, S., Chaumet-Riffaud, P., et al. Phase II study of a radiotherapy total dose increase in hypoxic lesions identified by F-miso PET/CT in patients with non-small cell lung carcinoma [RTEP5 study]. J Nucl Med 58, 1045-53 (2017).

19. Van Loon, J., Janssen, M. H. M., Öllers, M., et al. PET imaging of hypoxia using $\left[{ }^{18}\right.$ F]HX4: a phase I trial. Eur J Nucl Med Mol Imaging 37, 1663-8 (2010).

20. Dubois, L. J., Lieuwes, N. G., Janssen, M. H. M., et al. Preclinical evaluation and validation of $\left[{ }^{18} \mathrm{~F}\right] \mathrm{HX} 4$, a promising hypoxia marker for PET imaging. Proc Natl Acad Sci USA 108, 14620-5 (2011).

21. Peeters, S. G. J. A., Zegers, C. M. L., Lieuwes, N. G., et al. A comparative study of the hypoxia PET tracers $\left[{ }^{18} \mathrm{~F}\right]-\mathrm{HX} 4,\left[{ }^{18} \mathrm{~F}\right]-\mathrm{FAZA}$, and $\left[{ }^{18} \mathrm{~F}\right]-\mathrm{FMISO}$ in a preclinical tumor model. Int J Radiat Oncol Biol Phys 91, 351-9 (2015).

22. Van Baardwijk, A., Wanders, S., Boersma, L., et al. Mature results of an individualized radiation dose prescription study based on normal tissue constraints in stages I to III non-small-cell lung cancer. J Clin Oncol 28, 13806 (2010). 
23. Van Baardwijk, A., Reymen, B., Wanders, S., et al. Mature results of a phase II trial on individualised accelerated radiotherapy based on normal tissue constraints in concurrent chemo-radiation for stage III non-small cell lung cancer. Eur J Cancer 48, 2339-46 (2012).

24. Zegers, C. M. L., van Elmpt, W., Wierts, R., et al. Hypoxia imaging with $\left[{ }^{18} \mathrm{~F}\right] \mathrm{HX} 4$ PET in NSCLC patients: defining optimal imaging parameters. Radiother Oncol 109, 58-64 (2013).

25. Van Elmpt, W., Zegers, C. M. L., Reymen, B., et al. Multiparametric imaging of patient and tumour heterogeneity in non-small-cell lung cancer: quantification of tumour hypoxia, metabolism and perfusion. Eur J Nucl Med Mol Imaging 43, 240-8 (2016).

26. Saddi, K. A., Chefd'hotel, C. \& Cheriet, F. Large deformation registration of contrast-enhanced images with volume-preserving constraint in Medical Imaging 2007: Image Processing 6512 (SPIE, 2007), 651203.

27. Klein, S., Staring, M., Murphy, K., Viergever, M. A. \& Pluim, J. P. W. Elastix: a toolbox for intensity-based medical image registration. IEEE Trans Med Imaging 29, 196205 (2010).

28. Staring, M., Bakker, M. E., Stolk, J., Shamonin, D. P., Reiber, J. H. C. \& Stoel, B. C. Towards local progression estimation of pulmonary emphysema using CT. Med Phys 41, 021905 (2014).

29. Zegers, C. M. L., van Elmpt, W., Szardenings, K., et al. Repeatability of hypoxia PET imaging using $\left[{ }^{18} \mathrm{~F}\right] \mathrm{HX} 4$ in lung and head and neck cancer patients: a prospective multicenter trial. Eur J Nucl Med Mol Imaging 42, 1840-9 (2015).

30. Bradley, J. D., Paulus, R., Komaki, R., et al. Standard-dose versus high-dose conformal radiotherapy with concurrent and consolidation carboplatin plus paclitaxel with or without cetuximab for patients with stage IIIA or IIIB nonsmall-cell lung cancer (RTOG 0617): a randomised, two-by-two factorial phase 3 study. Lancet Oncol 16, 187-99 (2015).

31. Baumann, M., Krause, M., Overgaard, J., et al. Radiation oncology in the era of precision medicine. Nat Rev Cancer 16, 234-49 (2016).

32. Koo, T. R., Moon, S. H., Lim, Y. J., et al. The effect of tumor volume and its change on survival in stage III non-small cell lung cancer treated with definitive concurrent chemoradiotherapy. Radiat Oncol 9, 283 (2014).

33. Warner, A., Dahele, M., Hu, B., et al. Factors associated with early mortality in patients treated with concurrent chemoradiation therapy for locally advanced non-small cell lung cancer. Int J Radiat Oncol Biol Phys 94, 612-20 (2016). 
34. Na, F., Wang, J., Li, C., Deng, L., Xue, J. \& Lu, Y. Primary tumor standardized uptake value measured on F18-Fluorodeoxyglucose positron emission tomography is of prediction value for survival and local control in non-small-cell lung cancer receiving radiotherapy: meta-analysis. J Thorac Oncol 9, 834-42 (2014).

35. Goel, S., Duda, D. G., Xu, L., et al. Normalization of the vasculature for treatment of cancer and other diseases. Physiol Rev 91, 1071-121 (2011).

36. Lambin, P., Rios-Velazquez, E., Leijenaar, R., et al. Radiomics: extracting more information from medical images using advanced feature analysis. Eur J Cancer 48, 441-6 (2012).

37. Aerts, H. J. W. L., Velazquez, E. R., Leijenaar, R. T. H., et al. Decoding tumour phenotype by noninvasive imaging using a quantitative radiomics approach. Nat Commun 5, 4006 (2014). 


\section{Supplementary data}

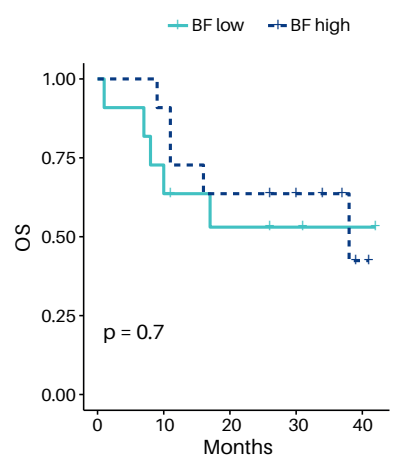

Number at risk

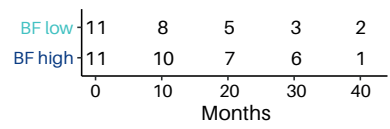

-BV low to BV high

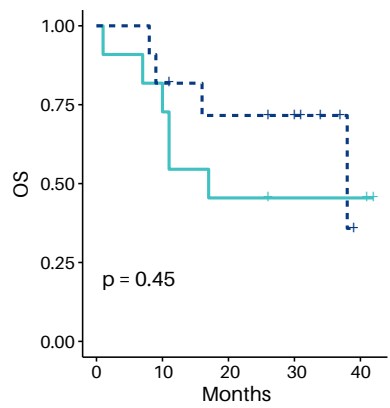

Number at risk

BV high -\begin{tabular}{ccccc}
-11 & 9 & 5 & 3 & 3 \\
0 & 9 & 7 & 6 & 0 \\
\hline 10 & $\begin{array}{c}10 \\
\text { Months }\end{array}$ & 30 & 40
\end{tabular}
+ BF low -tw B high

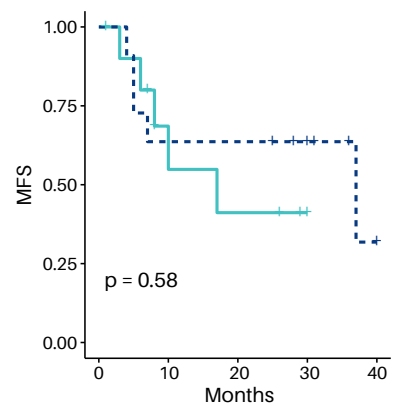

Number at risk

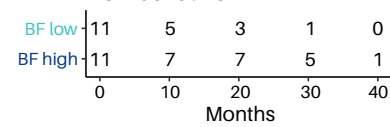

-BV low -t-BV high

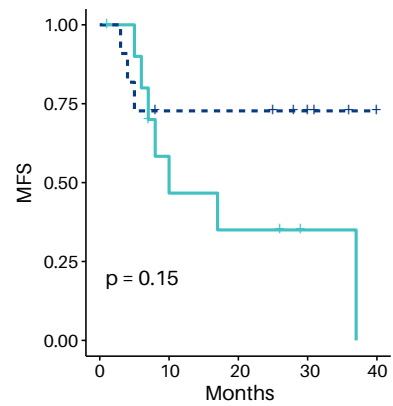

Number at risk

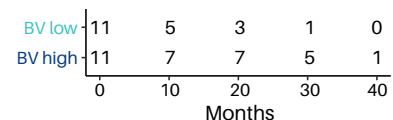

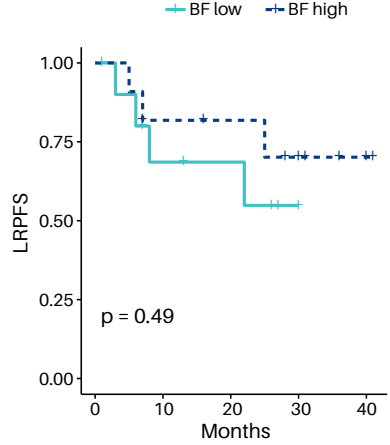

Number at risk

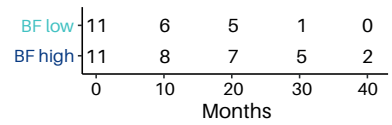

+BV low -tu BV high

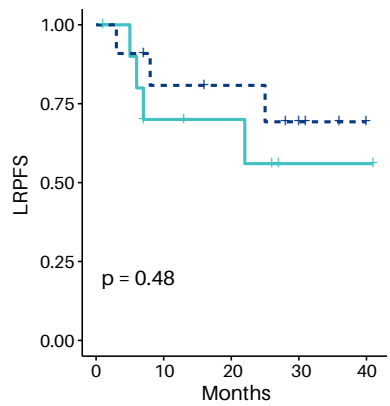

Number at risk

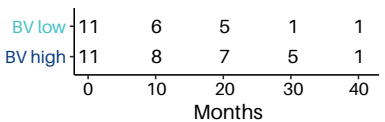

Figure S4.1. Prognostic value of baseline DCE-CT imaging of the primary tumour, with from left to right the overall survival (OS), metastasis-free survival (MFS), and locoregional progressionfree survival (LRPFS). The top row is tumour blood flow ( $>$ median), the bottom row is blood volume (> median). 


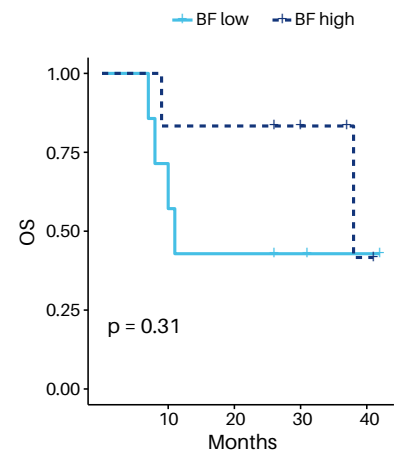

Number at risk
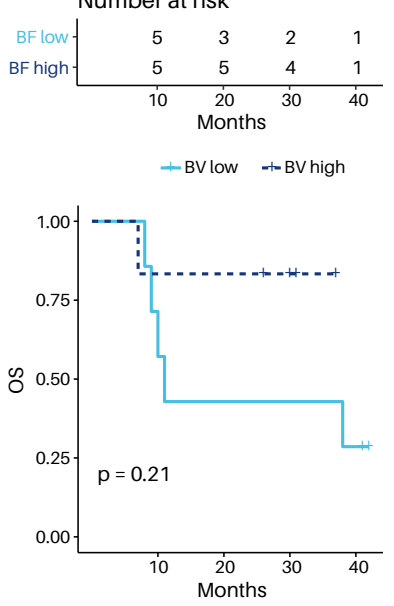

Number at risk

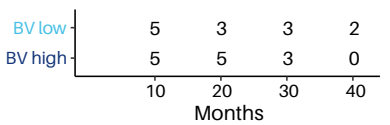

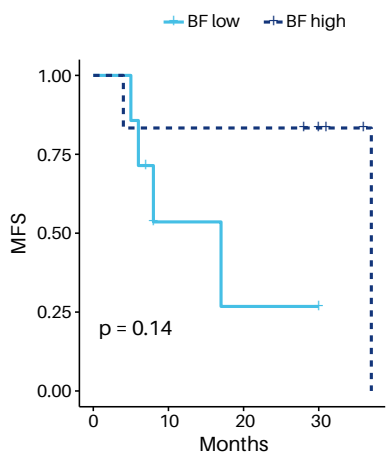

Number at risk

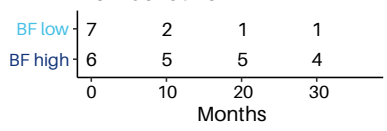

- BVlow + to BVhigh
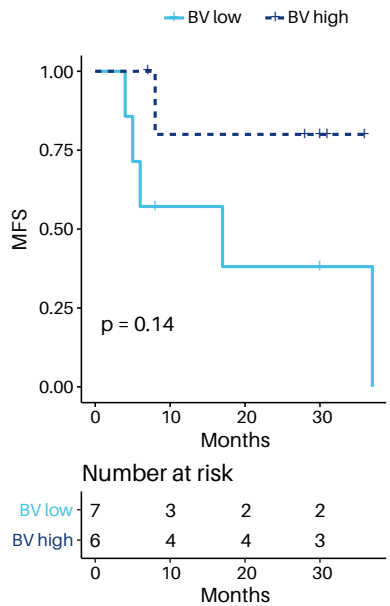

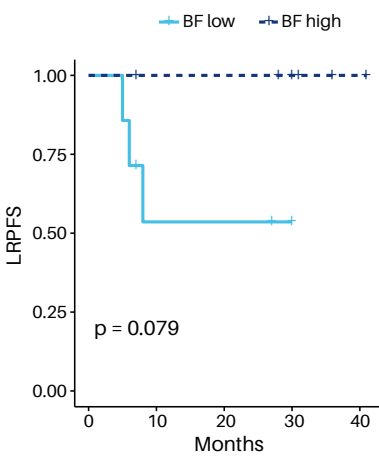

Number at risk

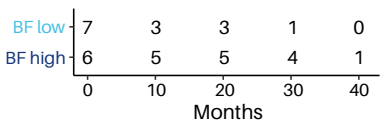

+ BV low + - B BVhigh
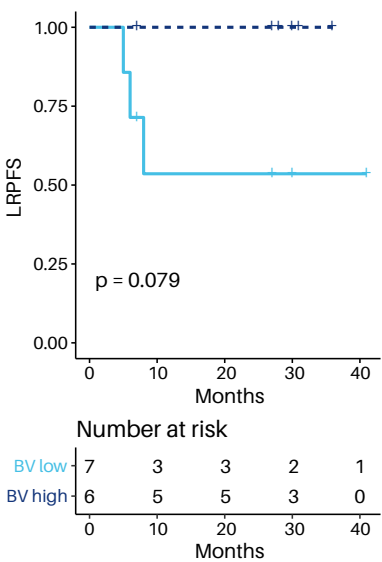

Figure S4.2. Prognostic value of DCE-CT imaging of the primary tumour after administration of nitroglycerin, with from left to right the overall survival (OS), metastasis-free survival (MFS), and locoregional progression-free survival (LRPFS). The top row is tumour blood flow (> median), the bottom row is blood volume (> median). 


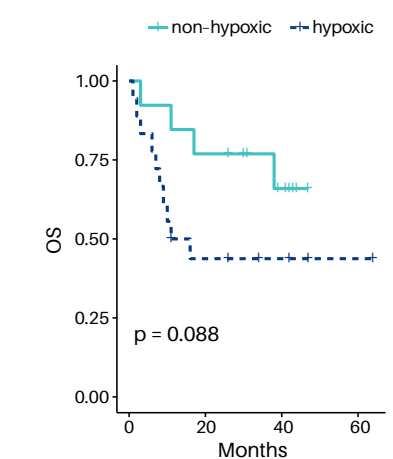

Number at risk

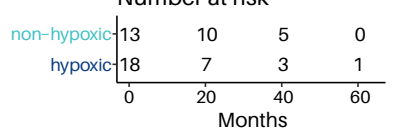

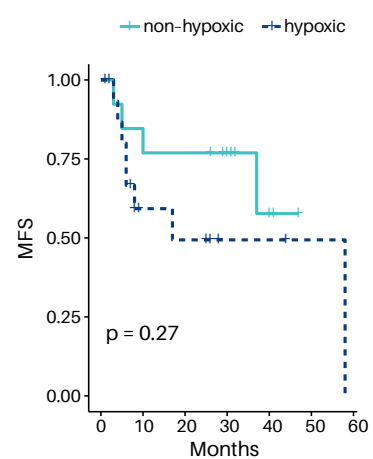

Number at risk

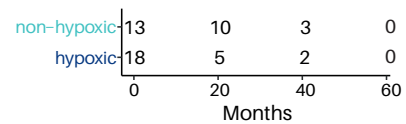

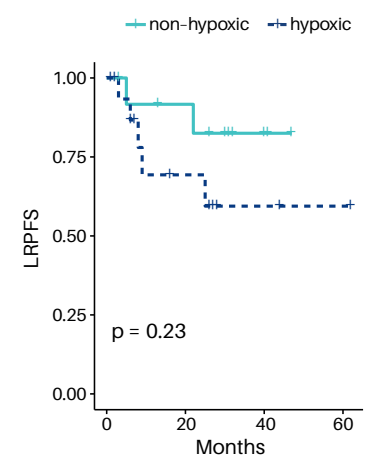

Number at risk

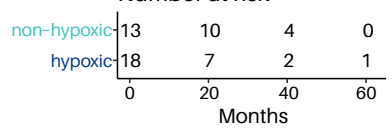

Figure S4.3. Prognostic value of baseline HX4 PET imaging of the primary tumour. Tumours with a tumour-to-background ratio $>1.4$ where categorized as hypoxic. From left to right the graphs show the overall survival (OS), metastasis-free survival (MFS), and locoregional progression-free survival (LRPFS).
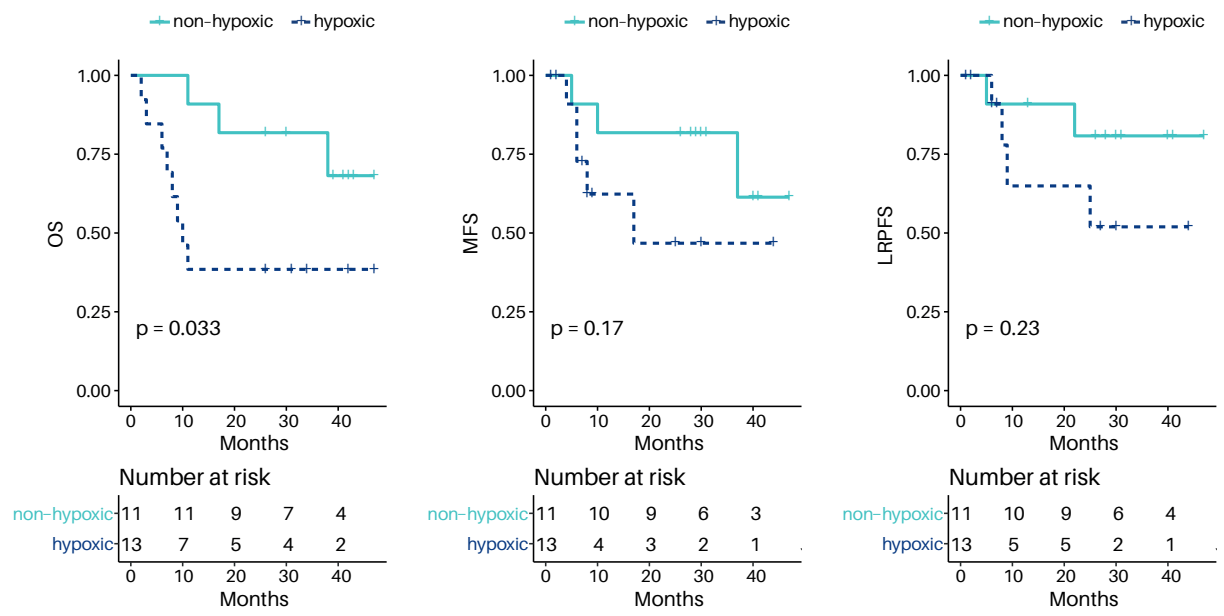

Figure S4.4. Prognostic value of HX4 PET imaging of the primary tumour after administration of nitroglycerin. Tumours with a tumour-to-background ratio $>1.4$ where categorized as hypoxic. From left to right the graphs show the overall survival (OS), metastasis-free survival (MFS), and locoregional progression-free survival (LRPFS). 



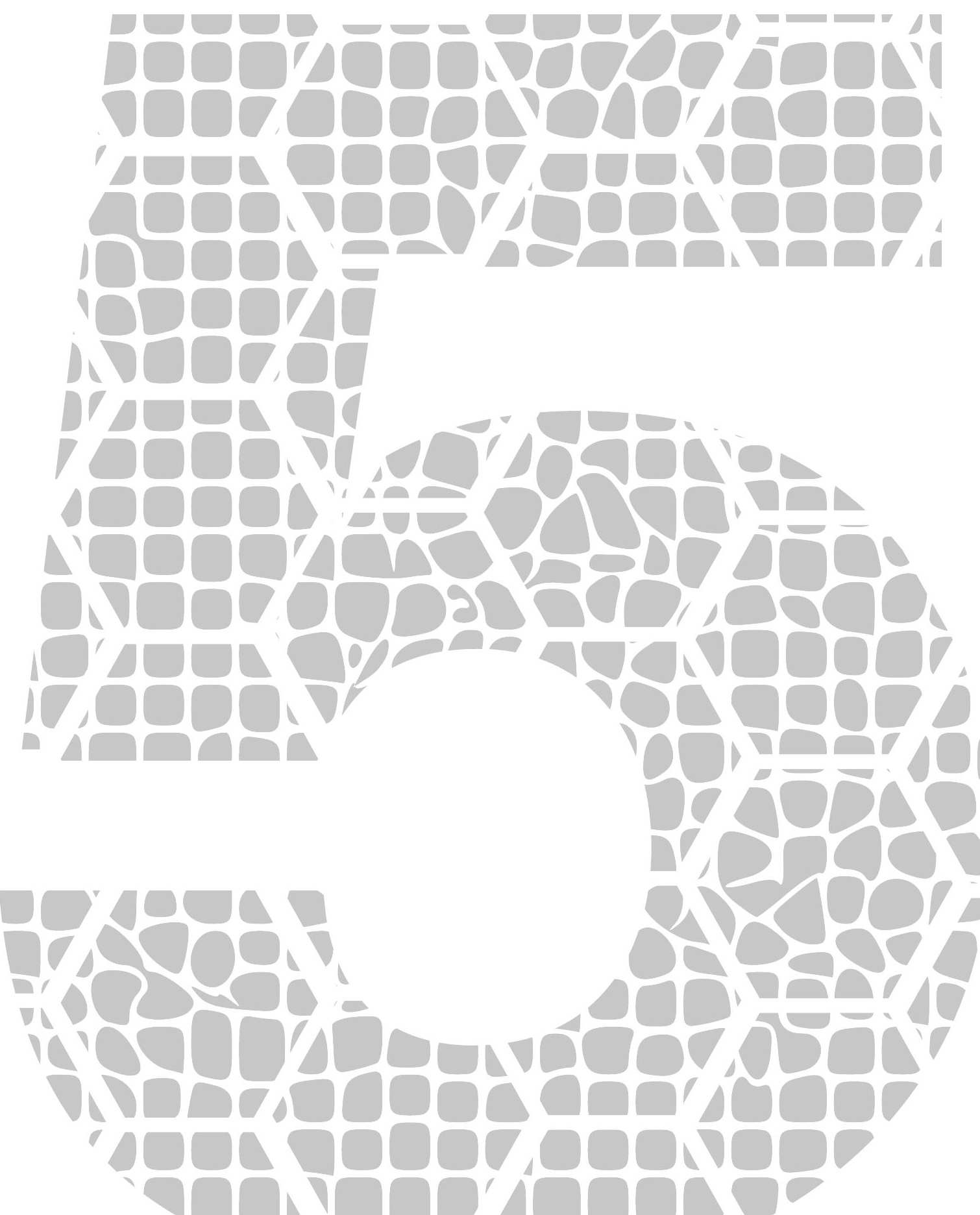




\section{PET-based dose painting in non- small cell lung cancer: comparing uniform dose escalation with boosting hypoxic and metabolically active subvolumes}

Aniek J.G. Even, Judith van der Stoep, Catharina M.L. Zegers, Bart Reymen, Esther G.C. Troost, Philippe Lambin, Wouter van Elmpt 


\begin{abstract}
Background and purpose We compared two imaging biomarkers for dose escalation in patients with advanced non-small cell lung cancer (NSCLC). Treatment plans boosting metabolically active subvolumes defined by FDG PET or hypoxic subvolumes defined by HX4 PET were compared with boosting the entire tumour.

Materials and methods Ten NSCLC patients underwent FDG and HX4 PET/CT scans prior to radiotherapy. Three isotoxic dose escalation plans were compared per patient: plan A, boosting the primary tumour $\left(\mathrm{PTV}_{\text {prim }}\right)$; plan B, boosting the subvolume with FDG PET uptake $>50 \%$ $\mathrm{SUV}_{\text {max }}\left(\mathrm{PTV}_{\mathrm{FDG}}\right)$; plan C, boosting the hypoxic volume with HX4 tumourto-background ratio $>1.4\left(\mathrm{PTV}_{\mathrm{HX} 4}\right)$.

Results Average boost volumes were $507 \mathrm{~cm}^{3} \pm 466 \mathrm{~cm}^{3}$ for $\mathrm{PTV}_{\text {prim }}$, $173 \mathrm{~cm}^{3} \pm 127 \mathrm{~cm}^{3}$ for PTV FDG and $114 \mathrm{~cm}^{3} \pm 73 \mathrm{~cm}^{3}$ for PTV $\mathrm{HX}_{4}$. The smaller PTV $\mathrm{HX}_{4}$ overlapped on average $87 \% \pm 16 \%$ with $\mathrm{PTV}_{\mathrm{FDG}}$. Prescribed dose was escalated to $87 \mathrm{~Gy} \pm 10 \mathrm{~Gy}$ for $\mathrm{PTV}$ prim, $107 \mathrm{~Gy} \pm 20 \mathrm{~Gy}$ for $\mathrm{PTV}_{\mathrm{FDG}}$, and $117 \mathrm{~Gy} \pm 15 \mathrm{~Gy}$ for $\mathrm{PTV}_{\mathrm{HX}}$, with comparable doses to the relevant organs at risk (OAR). Treatment plans are available online (https://www.cancerdata.org).

Conclusions Dose escalation based on metabolic subvolumes, hypoxic subvolumes and the entire tumour is feasible. The highest dose was achieved for hypoxia plans, without increasing dose to OAR. For most patients, boosting the metabolic subvolume also resulted in boosting the hypoxic volume, although to a lower dose, but not vice versa.
\end{abstract}




\section{Introduction}

The prognosis of patients with advanced stage non-small cell lung cancer (NSCLC) remains poor despite the use of new radiation techniques and addition of chemotherapy to radiotherapy [1]. Poor overall survival of patients treated with chemoradiotherapy is, besides distant metastases, negatively related to insufficient local tumour control. Increasing the dose to the tumour may improve local control, but escalating the dose to the whole tumour is currently limited by normal tissue toxicity $[2,3]$.

Instead of escalating the dose to the whole tumour, a more sophisticated approach may selectively increase dose to treatment resistant areas while maintaining the dose to other parts of the target volume: a concept called dose painting [4]. Tumours are known to be heterogeneous and biological properties, such as hypoxia, cell density, proliferation and vascularisation, vary throughout the tumour. As a consequence, some parts of the tumour are more treatment resistant than others [5,6]. This biological knowledge is integrated in dose painting to create highly personalized treatment plans. Dose can be prescribed to individual voxels (i.e. dose painting by numbers [7]) or to tumour subvolumes (i.e. dose painting by contours [4]). Dose painting by contours has the advantage that clinical treatment planning software can be used and a robust plan can be created by using margins to account for uncertainties. This latter approach is currently being tested in several clinical trials [8, 9].

Dose painting requires an imaging biomarker to determine subvolumes that are more treatment resistant and should therefore receive a higher dose. By selecting the appropriate positron emission tomography (PET) tracer, biological processes like hypoxia and glycolytic metabolism can be imaged. Hypoxia is a tumour characteristic that is known to be correlated with tumour aggressiveness and radioresistance $[10,11]$. The 2-nitroimidazole tracer 3-[ $\left.{ }^{18} \mathrm{~F}\right]$ fluoro-2-(4-((2-nitro-1H-imidazol-1-yl)methyl)1H-1,2,3-triazol-1-yl)propan-1-ol (HX4) is an example of a PET tracer whose ability to assess tumour hypoxia non-invasively has been validated [12-15]. Another biological process that can be assessed is tumour glucose metabolism, which is imaged with the commonly used tracer ${ }^{18} \mathrm{~F}$-fluorodeoxyglucose (FDG). For FDG PET, it has been shown that volumes of local relapse are correlated with high uptake regions on pretreatment images and are stable over time [16-19].

The question of which imaging surrogate should be used for dose painting is still under debate, but several surrogates have been and are being used in clinical trials $[8,9,20,21]$. FDG is commonly used and widely available; its high pretreatment uptake is related to local relapses. However, spatial correlation between uptake and local recurrences is not perfect. Aerts et al. [18] found that $30 \%$ of relapses were outside the high FDG region. On the other hand, hypoxia is known to be an important factor in chemotherapy and radiotherapy resistance. Although hypoxia imaging has only been 
used in clinical trials so far, these tracers are receiving more attention for dose painting purposes.

Zegers et al. [22] compared metabolic and hypoxic uptake patterns and found a good correlation between high uptake volumes of both tracers for most patients. For frequently used thresholds, the hypoxic volumes were generally smaller than the high metabolic uptake volumes. Consequently, creating a boost plan based on hypoxia imaging may reduce the boost volume and increase dose escalation levels even further. However, since margins have to be added in dose painting by contours to account for treatment delivery uncertainties, smaller high uptake subvolumes on PET do not automatically result in smaller planning boost volumes. Furthermore, Zegers et al. [22] described a partial mismatch between hypoxic and metabolic volumes in some patients. For those patients, it is a priori unknown what effect the selected boost volume with appropriate margins will have on the dose distributions of different dose painting plans.

Therefore, we compared different dose escalation strategies to determine the influence of the used imaging biomarker on achieved tumour dose levels. For each patient, we created an isotoxic dose painting plan boosting the FDG high uptake volume and a plan boosting the hypoxic volume. For comparison, a plan with a boost to the whole tumour was created. We evaluated the feasibility of these treatment plans together with dosimetrically achieved parameters for organs at risk dose and target volume. Furthermore, we evaluated the accuracy of a particular imaging surrogate for dose boosting on the coverage of the other biological subvolumes.

\section{Materials and methods}

\section{Patients}

NSCLC patients who were inoperable or had irresectable disease (cT2-T4, stage IB-III) were included in an ongoing phase II randomized clinical trial (NCT01024829). To be eligible, the primary tumour had to have a minimum diameter of $4 \mathrm{~cm}$ and maximum standardized uptake value $\left(\mathrm{SUV}_{\mathrm{max}}\right) \geq 5$ on pretreatment FDG PET [8]. The study was approved by the Medical Ethics Review Committee and all patients gave written informed consent. A dummy treatment plan was generated and a minimal dose of 72 Gy in 24 fractions (i.e. 3 Gy / fraction) to the planning target of the primary tumour

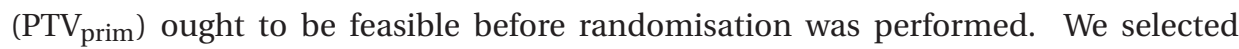
patients with hypoxic subvolumes as detected on HX4 PET for this study. 


\section{Image acquisition}

Patients were scheduled for FDG PET/CT and hypoxia HX4 PET/CT scans on different days, within the same week before radiotherapy. A pretreatment respiratory gated $4 \mathrm{D}$ FDG PET/CT was acquired using a Siemens Biograph 40 PET/CT scanner (Siemens Healthcare, Erlangen, Germany), according to the NEDPAS protocol [23]. HX4 PET/CT acquisition was performed four hours after injection of the hypoxia tracer on a Philips Gemini TF 64 scanner (Philips Healthcare, Best, the Netherlands) [13]. Patients were scanned in treatment position on a flat table top using the same fixation devices.

\section{Boost volumes}

Gross tumour volumes of the primary tumour $\left(\mathrm{GTV}_{\text {prim }}\right)$ and involved lymph nodes $\left(G_{\text {In }}\right)$ were delineated on the midventilation phase of the 4D FDG PET/CT scan by an experienced radiation oncologist. A $5 \mathrm{~mm}$ margin was added to the $\mathrm{GTV}_{\text {prim }}$ to include microscopic disease extension and create the clinical target volume $\left(\mathrm{CTV}_{\text {prim }}\right)$. An individualized margin, depending on the movement of the tumour on the $4 \mathrm{D}$ planning $\mathrm{CT}$, was added to the $\mathrm{CTV}_{\text {prim }}$ to create the planning target volume (PTV prim) [24]. For the lymph nodes, we used a $5 \mathrm{~mm}$ CTV and $5 \mathrm{~mm}$ PTV margin irrespective of the motion of the nodes.

The PTV $\mathrm{Prim}_{\text {was }}$ used as uniform boost planning target for plan A; additional structures were created to boost the metabolic and hypoxic subvolumes. Boosting the metabolic target $\left(\mathrm{GTV}_{\mathrm{FDG}}\right)$ was based on FDG PET/CT and defined as the region within GTV prim with an SUV above $50 \%$ of SUV $_{\max }$ (Plan B). Since the FDG PET/CT scan was used as planning CT, no additional registration of the PET to the CT had to be performed. Boosting the hypoxic volumes $\left(\mathrm{GTV}_{\mathrm{HX} 4}\right)$ was based on HX4 PET/CT (plan C). The HX4 PET/CT scan was registered rigidly to the planning FDG PET/CT scan using the treatment planning system (Eclipse version 11.0, Varian Medical Systems, Palo Alto, CA). We applied a bony anatomy match, followed by a soft tissue match with the primary tumour as region of interest. Background was defined as mean uptake in the aortic arch. Voxels with a tumour-to-background ratio (TBR) $>1.4$ within the $\mathrm{GTV}_{\text {prim }}$ were classified as $\mathrm{GTV}_{\mathrm{HX} 4}$. The TBR $>1.4$ cutoff value was chosen based on published research [12, 25-27]. For GTV $\mathrm{FDG}_{\text {and }}$ GTV $\mathrm{HX}_{4}$, we used the same individualized GTV-PTV margins to create PTV $_{\mathrm{FDG}}$ and $\mathrm{PTV}_{\mathrm{HX} 4}$ [8].

\section{Organs at risk}

Organs at risk (OAR) were delineated on the planning FDG PET/CT. Dose constraints were chosen according to the ongoing clinical trial protocol $[8,28]$ : lungs $\mathrm{D}_{\text {mean }}$ $<20$ Gy (corrected to EQD2); spinal cord $\mathrm{D}_{0.1}<51$ Gy (EQD2 $<52$ Gy); oesophagus $\mathrm{V}_{36}$ 
$<80 \%$ [29]; brachial plexus $\mathrm{D}_{0.1}<66$ Gy (EQD2 < 66 Gy); whole heart $\mathrm{D}_{\text {mean }}<46$ Gy (EQD2 $\left.\mathrm{D}_{\text {mean }}<46 \mathrm{~Gy}\right)$; planning organ at risk volume mediastinal structures (OAR + $5 \mathrm{~mm}$ margin) $\mathrm{D}_{0.1}<76$ Gy (EQD2 $<94 \mathrm{~Gy}$ ), where $\mathrm{D}_{\text {mean }}$ is the mean dose, $\mathrm{D}_{0.1}$ the dose delivered to $0.1 \%$ of the OAR, $\mathrm{V}_{36}$ the volume receiving $36 \mathrm{~Gy}$, and EQD2 the equivalent 2 Gy dose. For the biological dose calculation, we used an $\alpha$ / $\beta$ value of 3 Gy for the lungs, heart and mediastinal structures, 2 Gy for the spinal cord, and 10 Gy for the oesophagus.

\section{Treatment planning}

Experienced radiation technicians created three volumetric modulated arc therapy plans (VMAT; typically two half arcs for lateral tumours and two full arcs for medial tumours) for each patient using RapidArc (Eclipse version 11.0). Plans were created using a simultaneous integrated boost in 24 fractions. Doses were calculated with a type B dose calculation algorithm (AcurosXB-10.0). The prescribed dose was escalated until one or more of the OAR constraints was reached, or when a maximum dose of 129.6 Gy in 24 fractions in the $\mathrm{PTV}_{\text {boost }}$ volume (PTV prim for plan A; PTV ${ }_{\mathrm{FDG}}$ for plan B; $\mathrm{PTV}_{\mathrm{HX} 4}$ for plan $\mathrm{C}$ ) was achieved. An isotoxic planning strategy was applied to ensure equal lung toxicity. The target maximum mean lung dose difference between plans was 0.5 Gy. Plans with a higher mean lung dose were downscaled and reoptimized. This normalisation strategy has been shown to lead to similar mean doses in the $\mathrm{PTV}_{\text {prim }}$ [8].

The PTV boost was planned to have $99 \%$ of the volume covered by $90-115 \%$ of the prescribed dose. If there was overlap of less than $15 \%$ between PTV boost $_{\text {and OAR, a }}$ partial underdosage was accepted: $85 \%$ of $\mathrm{PTV}_{\text {boost }}$ was required to receive at least $90 \%$ of the prescribed dose where the dose in the overlap volume was escalated to $90 \%$ of the allowed OAR dose constraint. If this overlap was more than $15 \%$, no underdosing was accepted and $90 \%$ of the prescribed dose should cover $99 \%$ of the target, equal to the non-overlapping scenario. Lymph nodes were planned to receive 90-115\% of 66 Gy. If $\mathrm{PTV}_{\text {ln }}$ overlapped with $\mathrm{PTV}_{\text {prim, }}$, the $\mathrm{PTV}_{\text {prim }}$ was prioritized. Created treatment plans are available online at https://www.cancerdata.org.

\section{Analysis of the plans}

We calculated dose distributions and generated dose-volume histograms for the three treatment strategies. We compared prescribed and mean doses in the PTV $\mathrm{V}_{\text {prim }}$ between plans and calculated appropriate dose metrics for the OAR. We used a Wilcoxon signed rank test in SPSS (IBM Corp., Version 22.0, Armonk, NY) to compare prescribed doses and OAR doses between plan A, B and C. The significance level was adjusted for multiple comparisons (Bonferroni correction): a p-value $<0.017$ was assumed to be 
statistical significant. Finally, we calculated the overlap between $\mathrm{PTV}_{\mathrm{HX} 4}$ and $\mathrm{PTV}_{\mathrm{HX}}$ to evaluate how boosting one of the two subvolumes affected the other subvolume.

\section{Results}

Between September 2011 and August 2014, 35 NSCLC patients were included in the PET-boost clinical trial at our institute. Fourteen patients were not eligible because they did not receive a HX4 PET/CT which was part of the translational research of the trial and not mandatory for inclusion in the study, eight patients did not have a hypoxic tumour and in three patients dose escalation up to more than 72 Gy was not feasible. In total, ten patients were selected for this study. Patient and tumour characteristics are listed in Table 5.2.

The mean GTV prim was $199 \mathrm{~cm}^{3}$ (range: 32-853 $\mathrm{cm}^{3}$ ), mean GTV FDG $48 \mathrm{~cm}^{3}$ (range: $15-85 \mathrm{~cm}^{3}$ ) and mean $\mathrm{GTV}_{\mathrm{HX} 4} 30 \mathrm{~cm}^{3}$ (range: 5-58 $\mathrm{cm}^{3}$ ); see Supplementary Table S5.1. The respective mean planning target volumes were $507 \mathrm{~cm}^{3}$ (range: 149$1749 \mathrm{~cm}^{3}$ ), $173 \mathrm{~cm}^{3}$ (range: 53-484 $\mathrm{cm}^{3}$ ) and $114 \mathrm{~cm}^{3}$ (range: $32-273 \mathrm{~cm}^{3}$ ). Table 5.2 presents the boost volumes and overlap between those volumes. The volume encompassing both PTV $\mathrm{FDG}$ and PTV $\mathrm{HX}_{4}$ overlapped for $61 \%$ with PTV $\mathrm{FDG}$ and for $87 \%$ with $\mathrm{PTV}_{\mathrm{HX} 4}$. As shown in Table 5.2, $\mathrm{PTV}_{\mathrm{HX} 4}$ was smaller than $\mathrm{PTV}_{\mathrm{FDG}}$ for nine of the

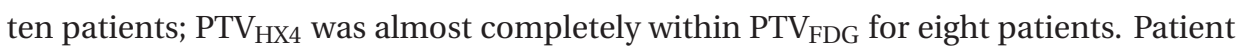
3 is an example of such a patient. In Figure 5.1 both PET scans and the delineated planning target volumes of patient 3 are shown. Patient 7 (also shown in Figure 5.1) is the only patient with a spatial mismatch between FDG and HX4 high uptake volumes.

It was feasible to generate plans with a boost to $\mathrm{PTV}_{\text {prim }}, \mathrm{PTV}_{\mathrm{FDG}}$ and $\mathrm{PTV}_{\mathrm{HX}}$ for all patients. The dose distributions of two patients, patient 3 and 7, are shown in Figure 5.1. The corresponding dose-volume histograms are displayed in Figure 5.2. For both patients, the histograms show that boosting the FDG volume also resulted in a boost to almost the entire hypoxic volume, while boosting hypoxia only increased the dose in part of the FDG high uptake volume.

For nine patients the highest dose was prescribed for plan C (see Supplementary

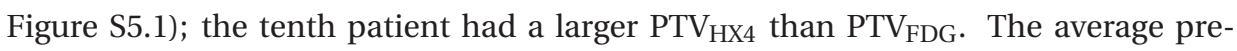
scribed doses to the boost volume were 87.1 Gy $\pm 10.1 \mathrm{~Gy}$ (plan A), 107.3 Gy $\pm 20.6 \mathrm{~Gy}$ (plan B) and 117.6 Gy $\pm 15.2 \mathrm{~Gy}$ (plan C). The prescribed doses for plans B ( $\mathrm{p}=0.005)$ and $C(p=0.005)$ were statistically significantly higher than for plan A. Prescribed doses and boost volumes per patient are listed in Table 5.1. Comparing prescribed doses with respect to boost volumes, Figure 5.3, shows that it is generally more difficult to prescribe a high dose to large boost volumes.

The isotoxic planning approach resulted in no significant differences in OAR doses (see Supplementary Table S5.2). Two examples of OAR dose-volume histograms are 

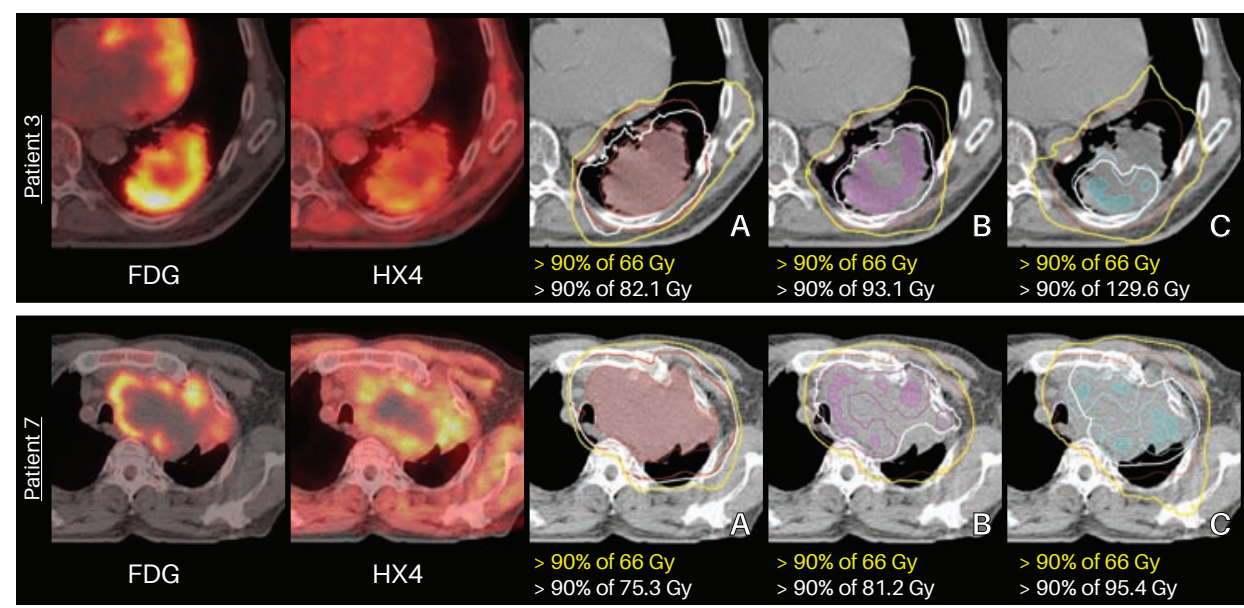

Figure 5.1. Example of pretreatment FDG and HX4 PET/CT scans. For patient 3 (top row) the $\mathrm{GTV}_{\mathrm{HX} 4}$ is smaller than the GTV FDG and the hypoxic volume is within the high FDG volume, which is the case for most patients. Patient 7 (bottom row) is the only patient with a spatial mismatch between FDG and HX4 uptake. The dose distributions of plans A, B and C are shown for the same patients. The red region is $\mathrm{GTV}_{\text {prim }}$, the magenta region $\mathrm{GTV}_{\mathrm{FDG}}$, and the cyan region GTV $\mathrm{HX}_{4}$. The line with corresponding colour represents $\mathrm{PTV}_{\text {prim }}, \mathrm{PTV}_{\mathrm{FDG}}$, and $\mathrm{PTV}_{\mathrm{HX} 4}$, respectively. The isodose line of $90 \%$ of the prescribed dose to the lymph nodes is shown in yellow and $90 \%$ of the prescribed dose to the boost region is shown in white.

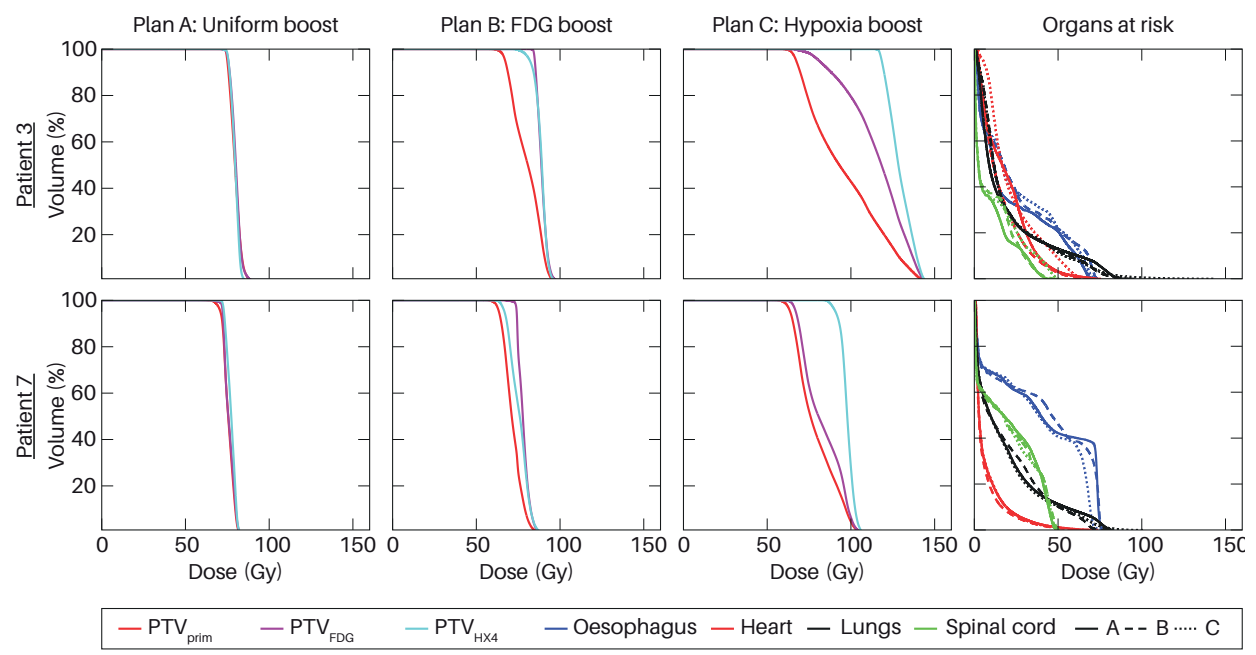

Figure 5.2. Dose-volume histograms for the planning target volumes for the primary tumour $\left(\mathrm{PTV}_{\text {prim }}\right)$, the high FDG volume $\left(\mathrm{PTV}_{\mathrm{FDG}}\right)$ and the hypoxic volume $\left(\mathrm{PTV}_{\mathrm{HX}}\right)$. In addition, the DVHs for the organs at risk are plotted. Plan A is displayed as a solid line, plan B as a dashed line and plan $\mathrm{C}$ as a dotted line for the organs at risk. The top row, patient 3 , and bottom row, patient 7, are the same patients as depicted in Figure 5.1. 
Table 5.1. Prescribed dose and mean dose to the planning target volume in the primary tumour $\left(\mathrm{PTV}_{\text {prim }}\right.$ ) for plan A (boost PTV $\mathrm{prim}_{\text {), plan B (boost PTV }}$ FDG) and plan C (boost $\mathrm{PTV}_{\mathrm{HX} 4}$ ). Doses were compared using a Wilcoxon signed rank test.

\begin{tabular}{|c|c|c|c|c|c|c|}
\hline \multirow[t]{2}{*}{ Patient } & \multicolumn{3}{|c|}{ Prescribed dose boost volumes (Gy) } & \multicolumn{3}{|c|}{$\mathrm{PTV}_{\text {prim }} \mathrm{D}_{\text {mean }}(\mathrm{Gy})$} \\
\hline & A & $\mathrm{B}$ & $\mathrm{C}$ & $\mathrm{A}$ & B & $\overline{\mathrm{C}}$ \\
\hline 1 & 83.0 & 110.0 & 129.6 & 81.8 & 85.9 & 87.1 \\
\hline 2 & 105.6 & 128.4 & 128.4 & 101.5 & 104.9 & 93.0 \\
\hline 3 & 82.1 & 93.1 & 129.6 & 78.4 & 78.2 & 92.0 \\
\hline 4 & 90.3 & 129.6 & 129.6 & 86.0 & 93.4 & 81.3 \\
\hline 5 & 78.0 & 90.7 & 101.0 & 76.5 & 80.2 & 86.9 \\
\hline 6 & 96.5 & 129.6 & 129.6 & 94.5 & 96.0 & 87.5 \\
\hline 7 & 75.3 & 81.2 & 95.4 & 75.6 & 71.6 & 78.9 \\
\hline 8 & 78.2 & 82.4 & 104.0 & 77.7 & 74.2 & 79.2 \\
\hline 9 & 83.9 & 98.3 & 99.8 & 82.4 & 86.9 & 85.1 \\
\hline 10 & 98.2 & 129.6 & 129.0 & 94.8 & 94.1 & 100.4 \\
\hline mean & $87 \pm 10$ & $107 \pm 21$ & $118 \pm 15$ & $85 \pm 9$ & $87 \pm 11$ & $87 \pm 7$ \\
\hline p-value compared to plan A & - & 0.005 & 0.005 & - & 0.203 & 0.386 \\
\hline p-value compared to plan B & 0.005 & - & 0.028 & 0.203 & - & 0.878 \\
\hline p-value compared to plan C & 0.005 & 0.028 & - & 0.386 & 0.878 & - \\
\hline
\end{tabular}

Abbreviations: PTV $_{\text {prim }}=$ planning target volume of the primary tumour; $\mathrm{D}_{\text {mean }}=$ mean dose.

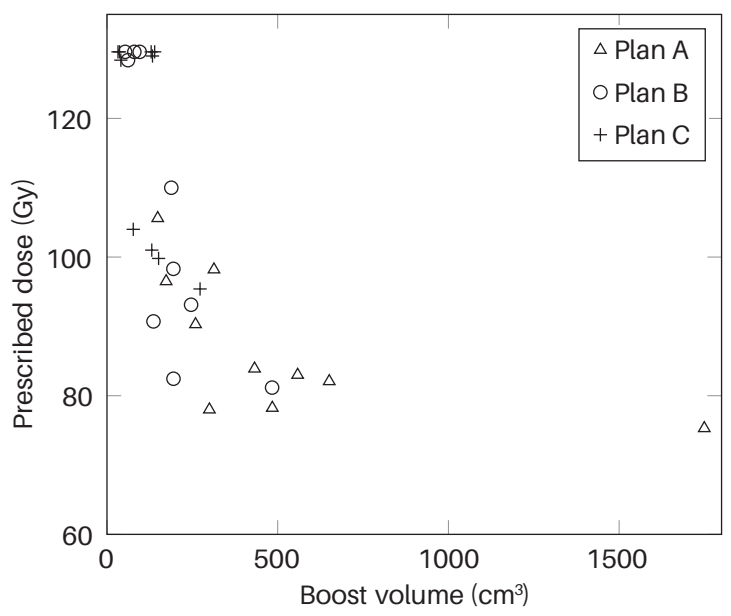

Figure 5.3. Prescribed dose to the boost region plotted as a function of the boost volume. Analysis for plan A (boost $\mathrm{PTV}_{\text {prim}}$ ), plan B (boost $\mathrm{PTV}_{\mathrm{FDG}}$ ) and plan C (boost $\mathrm{PTV}_{\mathrm{HX} 4}$ ). 
Table 5.2. Patient and tumour characteristics. Volumes of boost planning target for plan A ( $\left.P T V_{\text {prim }}\right)$, plan $B\left(\mathrm{PTV}_{\mathrm{FDG}}\right)$ and plan $\mathrm{C}\left(\mathrm{PTV} \mathrm{HX}_{4}\right)$, and the overlap between the planning volumes.

\begin{tabular}{|c|c|c|c|c|c|c|c|c|c|c|c|}
\hline \multirow[t]{2}{*}{ Patient } & \multirow[t]{2}{*}{ Gender } & \multirow[t]{2}{*}{ Age (years) } & \multirow[t]{2}{*}{ cTNM } & \multirow[t]{2}{*}{ Stage } & \multirow[t]{2}{*}{ Pathology } & \multicolumn{3}{|c|}{ Planning boost volume $\left(\mathrm{cm}^{3}\right)$} & \multicolumn{3}{|c|}{ Overlap PTV ${ }_{\text {FDG }}$ and PTV HX4 $_{4}$} \\
\hline & & & & & & A & B & $\overline{\mathrm{C}}$ & $\left(\mathrm{cm}^{3}\right)$ & $\left(\%\right.$ of PTV $\mathrm{FDG}_{\mathrm{F}}$ ) & $\left(\%\right.$ of $\left.\mathrm{PTV}_{\mathrm{HX}}\right)$ \\
\hline 1 & M & 64 & T4N2M0 & IIIb & Squamous cell carcinoma & 558.4 & 188.4 & 129.5 & 124.1 & 65.9 & 95.8 \\
\hline 2 & $\mathrm{~F}$ & 65 & T2N2M0 & IIIa & Adenocarcinoma & 148.6 & 61.6 & 40.7 & 38.7 & 62.8 & 95.2 \\
\hline 3 & M & 77 & T3N2M0 & IIIa & Large cell carcinoma & 651.0 & 246.4 & 139.7 & 122.0 & 49.5 & 87.3 \\
\hline 4 & M & 82 & T3N0M0 & IIb & Adenocarcinoma & 259.4 & 80.2 & 32.3 & 31.7 & 39.5 & 98.2 \\
\hline 5 & M & 66 & T2N3M0 & IIIb & Unknown & 300.0 & 136.2 & 131.2 & 118.8 & 87.3 & 90.6 \\
\hline 6 & M & 71 & T2N3M0 & IIIb & Adenocarcinoma & 173.3 & 53.2 & 36.8 & 35.8 & 67.3 & 97.2 \\
\hline 7 & M & 60 & T4N1M0 & IIIb & Adenocarcinoma & 1749.4 & 483.6 & 272.6 & 127.2 & 26.3 & 46.7 \\
\hline 8 & M & 65 & T3N2M0 & IIIa & Squamous cell carcinoma & 484.2 & 194.8 & 77.0 & 75.9 & 39.0 & 98.6 \\
\hline 9 & $\mathrm{~F}$ & 46 & T3N2M0 & IIIa & Adenocarcinoma & 432.2 & 194.5 & 151.2 & 137.6 & 70.8 & 91.0 \\
\hline 10 & M & 66 & T3N2M0 & IIIa & Adenocarcinoma & 313.3 & 95.8 & 133.3 & 94.3 & 98.4 & 70.7 \\
\hline mean & & $66 \pm 10$ & & & & $507 \pm 466$ & $173 \pm 127$ & $114 \pm 73$ & $91 \pm 42$ & $61 \pm 22$ & $87 \pm 16$ \\
\hline
\end{tabular}

Abbreviations: PTV $_{\text {prim }}=$ planning target volume of the primary tumour; PTV $_{\mathrm{FDG}}=$ planning target volume of the high FDG subvolume, $\mathrm{PTV}$ HX4 $=$ PTV of the

hypoxic subvolume. 
shown in Figure 5.2. Prescribed dose was limited either by the mediastinal structures (seven patients), or mean lung dose (three patients).

\section{Discussion}

We performed a dosimetric comparison of three clinically feasible dose escalation plans for ten NSCLC patients: boosting FDG and HX4 high uptake volumes was compared with boosting the entire tumour. The size of the boost volume appears to be an important factor for the dose level that can be prescribed. Hypoxia planning volumes were generally smaller than FDG boost volumes, resulting in less overlap between target and surrounding OAR. In two cases, underdosage of the boost volumes was allowed in the hypoxia plan because overlap with the OAR was less than the predefined threshold (i.e. $15 \%$ of the volume), but it was not tolerated for the other two plans. This facilitated further dose escalation in the hypoxia boost plan.

To our knowledge, this is the first study showing the feasibility of boosting hypoxia in NSCLC. This approach was already proven feasible in head and neck cancer [30-33]. It is important to note that not all patients were eligible for hypoxia boosting; $50 \%$ of the patients had hypoxic tumours. We do not expect that this approach is suitable for all patients; patient selection is essential and treatment should be adapted for every patient. Patients with non-hypoxic tumours have generally a better prognosis [34-38] and probably do not need an aggressive treatment as presented. Based on pretreatment hypoxia and FDG PET/CT scans it can be decided to boost hypoxic or FDG subvolumes, or a combination. For most patients the largest part of the hypoxic volume was located inside the high FDG volume. As a consequence, boosting FDG will also boost the hypoxia, but a boost to the hypoxic volume will not escalate the dose in the entire FDG volume. For patients without a hypoxia scan, FDG PET imaging can be used as a surrogate for boosting hypoxia; however, less dose escalation can be achieved.

We used commonly applied thresholds to determine FDG and HX4 high uptake subvolumes, although these thresholds are arbitrarily defined. The FDG threshold is already used in a clinical trial [8], whereas the HX4 threshold is based on literature [12, 25-27]. Zegers et al. [22] showed that the proportional overlap of the tracers is rather stable for varying thresholds. That suggests that the FDG plan will still boost the hypoxia volume with different thresholds, but not vice versa. The different thresholds are likely to have the greatest impact on the height of the prescribed dose levels, as these are linked to boost volume (Figure 5.3).

An important aspect that has to be considered in hypoxia dose painting is stability of the target over time. Small studies testing temporal stability of hypoxia in NSCLC have so far found inconclusive results. Some studies have observed stable hypoxia 
$[39,40]$, whereas others have reported a decrease in hypoxia [26, 41], or a mix of stable and dynamic hypoxia [42]. Lin et al. [43] showed in head and neck cancer that when there is a spatial shift, dose painting of the initial subvolume still results in improved equivalent uniform dose on a later scan. For pretreatment FDG PET/CT imaging, it is known that most local relapses were within the FDG high uptake region. However, it is still unknown whether pretreatment hypoxic regions also correlate with the locations of local relapses. Because high correlations are observed between FDG high uptake areas and hypoxic areas on pretreatment scans, we hypothesize that local recurrences will primarily occur in these hypoxic regions. It is essential to confirm this hypothesis before applying hypoxia boosting in clinical practice. This will in the future be assessed in the PET-boost clinical trial.

This study used a dose painting by contours approach. Compared to dose painting by numbers, this approach is easier to implement in clinical treatment planning software. Furthermore, it includes a safety PTV margin to tackle small deviations in target subvolume definition.

Finally, there is still debate about the applicability and safety of dose escalation in NSCLC. Although multiple clinical trials suggest dose escalations could improve overall survival [44-46], a large randomized phase III trial showed an unexpected lower survival for the group that received a higher dose [47]. This unexpected outcome may result from various causes including longer overall treatment time, increased cardiac toxicity, or compromises in defining the PTV for the high dose group [48]. Alternatively, the results may suggest that standard dose escalation is not the way forward and more sophisticated dose redistribution techniques are necessary to improve local survival.

In conclusion, selective boosting of subvolumes based on FDG or hypoxia is feasible and increases the prescribed dose compared to whole tumour boosting, without increasing the dose to the organs at risk.

\section{Acknowledgements}

Authors acknowledge financial support from EU 7th framework program (ARTFORCE), Kankeronderzoekfonds Limburg from the Health Foundation Limburg and the Dutch Cancer Society (KWF MAC 2011-5020 and KWF MAC 2011-4970). This research is also supported by the Dutch technology Foundation STW (grant $n^{\circ} 10696$ DuCAT), which is the applied science division of NWO, and the Technology Programme of the Ministry of Economic Affairs. 


\section{References}

1. Aupérin, A., Le Péchoux, C., Rolland, E., et al. Meta-analysis of concomitant versus sequential radiochemotherapy in locally advanced non-small-cell lung cancer. J Clin Oncol 28, 2181-90 (2010).

2. Kong, F. M., Ten Haken, R. K., Schipper, M. J., et al. High-dose radiation improved local tumor control and overall survival in patients with inoperable/unresectable non-small-cell lung cancer: long-term results of a radiation dose escalation study. Int J Radiat Oncol Biol Phys 63, 324-33 (2005).

3. Machtay, M., Bae, K., Movsas, B., et al. Higher biologically effective dose of radiotherapy is associated with improved outcomes for locally advanced nonsmall cell lung carcinoma treated with chemoradiation: an analysis of the Radiation Therapy Oncology Group. Int J Radiat Oncol Biol Phys 82, 425-34 (2012).

4. Ling, C. C., Humm, J., Larson, S., et al. Towards multidimensional radiotherapy (MD-CRT): biological imaging and biological conformality. Int J Radiat Oncol Biol Phys 47, 551-60 (2000).

5. Lambin, P., Petit, S. F., Aerts, H. J. W. L., et al. The ESTRO Breur lecture 2009. From population to voxel-based radiotherapy: exploiting intra-tumour and intra-organ heterogeneity for advanced treatment of non-small cell lung cancer. Radiother Oncol 96, 145-52 (2010).

6. Petit, S. F., Dekker, A. L. A. J., Seigneuric, R., et al. Intra-voxel heterogeneity influences the dose prescription for dose-painting with radiotherapy: a modelling study. Phys Med Biol 54, 2179-96 (2009).

7. Bentzen, S. M. Theragnostic imaging for radiation oncology: dose-painting by numbers. The Lancet Oncology 6, 112-7 (2005).

8. Van Elmpt, W., De Ruysscher, D., van der Salm, A., et al. The PET-boost randomised phase II dose-escalation trial in non-small cell lung cancer. Radiother Oncol 104, 67-71 (2012).

9. Madani, I., Duthoy, W., Derie, C., et al. Positron Emission Tomography-guided, focal-dose escalation using intensity-modulated radiotherapy for head and neck cancer. Int J Radiat Oncol Biol Phys 68, 126-35 (2007).

10. Bollineni, V. R., Wiegman, E. M., Pruim, J., Groen, H. J. M. \& Langendijk, J. A. Hypoxia imaging using Positron Emission Tomography in non-small cell lung cancer: implications for radiotherapy. Cancer Treat Rev 38, 1027-32 (2012). 
11. Zips, D., Böke, S., Kroeber, T., et al. Prognostic value of radiobiological hypoxia during fractionated irradiation for local tumor control. Strahlenther Onkol 187, 306-10 (2011).

12. Dubois, L. J., Lieuwes, N. G., Janssen, M. H. M., et al. Preclinical evaluation and validation of $\left[{ }^{18} \mathrm{~F}\right] \mathrm{HX} 4$, a promising hypoxia marker for PET imaging. Proc Natl Acad Sci USA 108, 14620-5 (2011).

13. Zegers, C. M. L., van Elmpt, W., Wierts, R., et al. Hypoxia imaging with $\left[{ }^{18} \mathrm{~F}\right] \mathrm{HX} 4$ PET in NSCLC patients: defining optimal imaging parameters. Radiother Oncol 109, 58-64 (2013).

14. Peeters, S. G. J. A., Zegers, C. M. L., Lieuwes, N. G., et al. A comparative study of the hypoxia PET tracers $\left[{ }^{18} \mathrm{~F}\right]-\mathrm{HX} 4,\left[{ }^{18} \mathrm{~F}\right]-\mathrm{FAZA}$, and $\left[{ }^{18} \mathrm{~F}\right]-\mathrm{FMISO}$ in a preclinical tumor model. Int J Radiat Oncol Biol Phys 91, 351-9 (2015).

15. Van Loon, J., Janssen, M. H. M., Öllers, M., et al. PET imaging of hypoxia using $\left[{ }^{18}\right.$ F]HX4: a phase I trial. Eur J Nucl Med Mol Imaging 37, 1663-8 (2010).

16. Aerts, H. J. W. L., Bosmans, G. M., van Baardwijk, A. A. W., et al. Stability of ${ }^{18} \mathrm{~F}-$ deoxyglucose uptake locations within tumor during radiotherapy for NSCLC: a prospective study. Int J Radiat Oncol Biol Phys 71, 1402-7 (2008).

17. Abramyuk, A., Tokalov, S., Zöphel, K., et al. Is pre-therapeutical FDG-PET/CT capable to detect high risk tumor subvolumes responsible for local failure in nonsmall cell lung cancer? Radiother Oncol 91, 399-404 (2009).

18. Aerts, H. J. W. L., van Baardwijk, A. A. W., Petit, S. F., et al. Identification of residual metabolic-active areas within individual NSCLC tumours using a preradiotherapy ${ }^{18}$ Fluorodeoxyglucose-PET-CT scan. Radiother Oncol 91, 386-92 (2009).

19. Aerts, H. J. W. L., Lambin, P. \& De Ruysscher, D. FDG for dose painting: a rational choice. Radiother Oncol 97, 163-4 (2010).

20. Würschmidt, F., Petersen, C., Wahl, A., Dahle, J. \& Kretschmer, M. $\left[{ }^{18} \mathrm{~F}\right]-$ fluoroethylcholine-PET/CT imaging for radiation treatment planning of recurrent and primary prostate cancer with dose escalation to PET/CT-positive lymph nodes. Radiat Oncol 6, 44 (2011).

21. Troost, E. G. C., Bussink, J., Hoffmann, A. L., Boerman, O. C., Oyen, W. J. G. \& Kaanders, J. H. A. M. ${ }^{18}$ F-FLT PET/CT for early response monitoring and dose escalation in oropharyngeal tumors. J Nucl Med 51, 866-74 (2010).

22. Zegers, C. M. L., van Elmpt, W., Reymen, B., et al. In vivo quantification of hypoxic and metabolic status of NSCLC tumors using $\left[{ }^{18} \mathrm{~F}\right] \mathrm{HX} 4$ and $\left[{ }^{18} \mathrm{~F}\right] \mathrm{FDG}$-PET/CT imaging. Clin Cancer Res 20, 6389-97 (2014). 
23. Boellaard, R., Oyen, W. J. G., Hoekstra, C. J., et al. The Netherlands protocol for standardisation and quantification of FDG whole body PET studies in multicentre trials. Eur J Nucl Med Mol Imaging 35, 2320-33 (2008).

24. Van Herk, M., Remeijer, P. \& Lebesque, J. V. Inclusion of geometric uncertainties in treatment plan evaluation. Int J Radiat Oncol Biol Phys 52, 1407-22 (2002).

25. Dubois, L., Landuyt, W., Haustermans, K., et al. Evaluation of hypoxia in an experimental rat tumour model by $\left[{ }^{18} \mathrm{~F}\right]$ fluoromisonidazole PET and immunohistochemistry. Br J Cancer 91, 1947-54 (2004).

26. Koh, W. J., Bergman, K. S., Rasey, J. S., et al. Evaluation of oxygenation status during fractionated radiotherapy in human nonsmall cell lung cancers using [F18]fluoromisonidazole positron emission tomography. Int J Radiat Oncol Biol Phys 33, 391-8 (1995).

27. Rasey, J. S., Koh, W. J., Evans, M. L., et al. Quantifying regional hypoxia in human tumors with positron emission tomography of $\left[{ }^{18} \mathrm{~F}\right]$ fluoromisonidazole: a pretherapy study of 37 patients. Int J Radiat Oncol Biol Phys 36, 417-28 (1996).

28. Van Diessen, J., Belderbos, J., Sonke, J. J., et al. Very high radiation dose escalation in NSCLC does not lead to unexpected toxicity: a planned toxicity analysis of the PET-boost study (NCT01024829). J Thorac Oncol 8, Supplement 2, S1135 (2013).

29. Belderbos, J., Heemsbergen, W., Hoogeman, M., Pengel, K., Rossi, M. \& Lebesque, J. Acute esophageal toxicity in non-small cell lung cancer patients after high dose conformal radiotherapy. Radiother Oncol 75, 157-64 (2005).

30. Chang, J. H., Wada, M., Anderson, N. J., et al. Hypoxia-targeted radiotherapy dose painting for head and neck cancer using ${ }^{18} \mathrm{~F}$-FMISO PET: a biological modeling study. Acta Oncol 52, 1723-9 (2013).

31. Choi, W., Lee, S. W., Park, S. H., et al. Planning study for available dose of hypoxic tumor volume using fluorine-18-labeled fluoromisonidazole positron emission tomography for treatment of the head and neck cancer. Radiother Oncol 97, 17682 (2010).

32. Hendrickson, K., Phillips, M., Smith, W., Peterson, L., Krohn, K. \& Rajendran, J. Hypoxia imaging with [F-18] FMISO-PET in head and neck cancer: potential for guiding intensity modulated radiation therapy in overcoming hypoxia-induced treatment resistance. Radiother Oncol 101, 369-75 (2011).

33. Toma-Dasu, I., Uhrdin, J., Antonovic, L., et al. Dose prescription and treatment planning based on FMISO-PET hypoxia. Acta Oncol 51, 222-30 (2012).

34. Horsman, M. R., Mortensen, L. S., Petersen, J. B., Busk, M. \& Overgaard, J. Imaging hypoxia to improve radiotherapy outcome. Nat Rev Clin Oncol 9, 674-87 (2012). 
35. Dehdashti, F., Mintun, M. A., Lewis, J. S., et al. In vivo assessment of tumor hypoxia in lung cancer with ${ }^{60} \mathrm{Cu}$-ATSM. Eur J Nucl Med Mol Imaging 30, 84450 (2003).

36. Eschmann, S. M., Paulsen, F., Reimold, M., et al. Prognostic impact of hypoxia imaging with ${ }^{18} \mathrm{~F}$-misonidazole PET in non-small cell lung cancer and head and neck cancer before radiotherapy. J Nucl Med 46, 253-60 (2005).

37. Li, L., Hu, M., Zhu, H., Zhao, W., Yang, G. \& Yu, J. Comparison of $\left[{ }^{18} \mathrm{~F}\right]-$ fluoroerythronitroimidazole and $\left[{ }^{18} \mathrm{~F}\right]$-fluorodeoxyglucose positron emission tomography and prognostic value in locally advanced non-small-cell lung cancer. Clin Lung Cancer 11, 335-40 (2010).

38. Peeters, S. G. J. A., Zegers, C. M. L., Yaromina, A., Van Elmpt, W., Dubois, L. \& Lambin, P. Current preclinical and clinical applications of hypoxia PET imaging using 2-nitroimidazoles. Q J Nucl Med Mol Imaging 59, 39-57 (2015).

39. Vera, P., Bohn, P., Edet-Sanson, A., et al. Simultaneous positron emission tomography (PET) assessment of metabolism with ${ }^{18}$ F-fluoro-2-deoxy-d-glucose (FDG), proliferation with ${ }^{18} \mathrm{~F}$-fluoro-thymidine (FLT), and hypoxia with ${ }^{18}$ fluoromisonidazole (F-miso) before and during radiotherapy in patients with nonsmall-cell lung cancer (NSCLC): a pilot study. Radiother Oncol 98, 109-16 (2011).

40. Bradshaw, T. J., Yip, S., Jallow, N., Forrest, L. J. \& Jeraj, R. Spatiotemporal stability of $\mathrm{Cu}$-ATSM and FLT positron emission tomography distributions during radiation therapy. Int J Radiat Oncol Biol Phys 89, 399-405 (2014).

41. Trinkaus, M. E., Blum, R., Rischin, D., et al. Imaging of hypoxia with ${ }^{18}$ F-FAZA PET in patients with locally advanced non-small cell lung cancer treated with definitive chemoradiotherapy. J Med Imaging Radiat Oncol 57, 475-81 (2013).

42. Bollineni, V. R., Koole, M. J. B., Pruim, J., et al. Dynamics of tumor hypoxia assessed by ${ }^{18} \mathrm{~F}$-FAZA PET/CT in head and neck and lung cancer patients during chemoradiation: Possible implications for radiotherapy treatment planning strategies. Radiother Oncol 113, 198-203 (2014).

43. Lin, Z., Mechalakos, J., Nehmeh, S., et al. The influence of changes in tumor hypoxia on dose-painting treatment plans based on ${ }^{18} \mathrm{~F}$-FMISO positron emission tomography. Int J Radiat Oncol Biol Phys 70, 1219-28 (2008).

44. Rengan, R., Rosenzweig, K. E., Venkatraman, E., et al. Improved local control with higher doses of radiation in large-volume stage III non-small-cell lung cancer. Int J Radiat Oncol Biol Phys 60, 741-7 (2004). 
45. Van Baardwijk, A., Reymen, B., Wanders, S., et al. Mature results of a phase II trial on individualised accelerated radiotherapy based on normal tissue constraints in concurrent chemo-radiation for stage III non-small cell lung cancer. Eur J Cancer 48, 2339-46 (2012).

46. Van Baardwijk, A., Wanders, S., Boersma, L., et al. Mature results of an individualized radiation dose prescription study based on normal tissue constraints in stages I to III non-small-cell lung cancer. J Clin Oncol 28, 13806 (2010).

47. Bradley, J. D., Paulus, R., Komaki, R., et al. Standard-dose versus high-dose conformal radiotherapy with concurrent and consolidation carboplatin plus paclitaxel with or without cetuximab for patients with stage IIIA or IIIB nonsmall-cell lung cancer (RTOG 0617): a randomised, two-by-two factorial phase 3 study. Lancet Oncol 16, 187-99 (2015).

48. Cox, J. D. Are the results of RTOG 0617 mysterious? Int J Radiat Oncol Biol Phys 82, 1042-4 (2012). 


\section{Supplementary data}

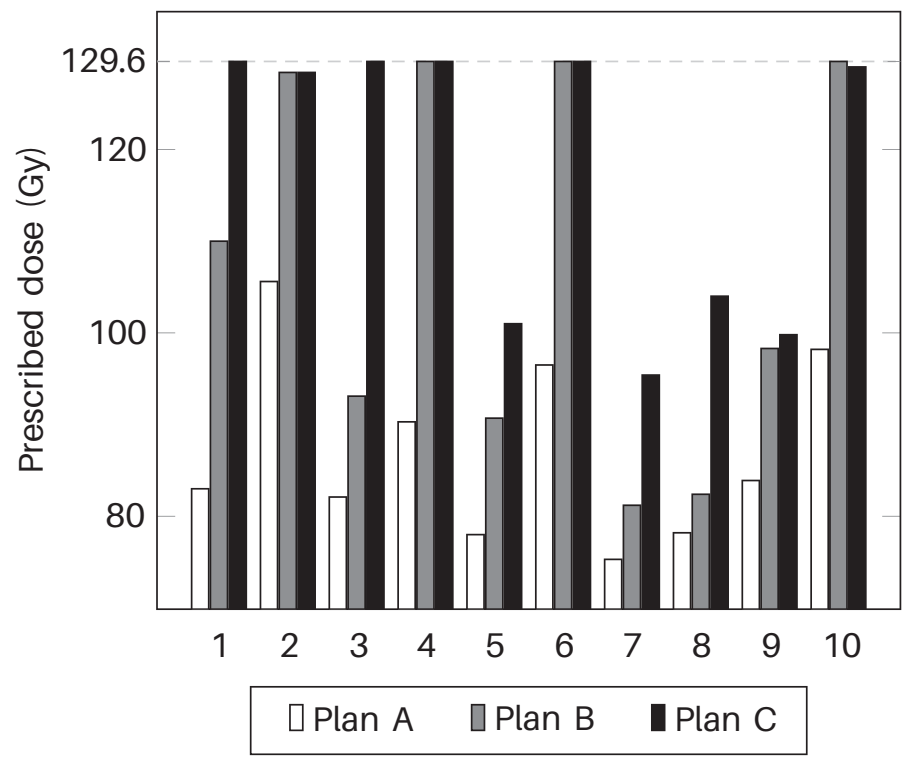

Figure S5.1. Prescribed dose to the boost volume per patient for plan A (boost $\mathrm{PTV}_{\text {prim }}$ ) plan B (boost $\mathrm{PTV}_{\mathrm{FDG}}$ ) and plan C (boost $\mathrm{PTV}_{\mathrm{HX}}$ ). The dose is escalated up to a maximum of $129.6 \mathrm{~Gy}$. 
Table S5.1. Gross tumour volume of the primary tumour $\left(\mathrm{GTV}_{\text {prim }}\right)$, the FDG high uptake volume $\left(\mathrm{GTV}_{\mathrm{FDG}}\right)$ and the hypoxic volume $\left(\mathrm{GTV}_{\mathrm{HX} 4}\right)$. The overlap between $\mathrm{GTV}_{\mathrm{FDG}}$ and $\mathrm{GTV}_{\mathrm{HX} 4}$ is also listed.

\begin{tabular}{|c|c|c|c|c|c|c|c|c|c|}
\hline \multirow[t]{2}{*}{ Patient } & \multirow{2}{*}{$\frac{\mathrm{GTV}_{\text {prim }}}{\left(\mathrm{cm}^{3}\right)}$} & \multicolumn{2}{|r|}{ GTV $_{\text {FDG }}$} & \multicolumn{2}{|r|}{$\mathrm{GTV}_{\mathrm{HX} 4}$} & \multicolumn{4}{|c|}{ Overlap GTV $_{\mathrm{FDG}}$ and GTV $\mathrm{HX}_{\mathrm{H} 4}$} \\
\hline & & $\left(\mathrm{cm}^{3}\right)$ & $\left(\%\right.$ of $\left.\mathrm{GTV}_{\text {prim }}\right)$ & $\left(\mathrm{cm}^{3}\right)$ & $\left(\%\right.$ of $\left.\mathrm{GTV}_{\text {prim }}\right)$ & $\left(\mathrm{cm}^{3}\right)$ & (\% of $\left.\mathrm{GTV}_{\text {prim }}\right)$ & $\left(\mathrm{OF}_{\mathrm{FDG}}\right)$ & $\left(\mathrm{OF}_{\mathrm{HX} 4}\right)$ \\
\hline 1 & 213.6 & 76.8 & 35.9 & 36.4 & 17.1 & 32.1 & 15.0 & 0.4 & 0.9 \\
\hline 2 & 32.5 & 15.5 & 47.6 & 7.7 & 23.6 & 7.0 & 21.6 & 0.5 & 0.9 \\
\hline 3 & 295.2 & 74.2 & 25.1 & 19.7 & 6.7 & 14.8 & 5.0 & 0.2 & 0.8 \\
\hline 4 & 62.9 & 19.4 & 30.8 & 5.0 & 8.0 & 4.7 & 7.5 & 0.2 & 0.9 \\
\hline 5 & 78.0 & 44.2 & 56.7 & 47.9 & 61.4 & 37.4 & 48.0 & 0.8 & 0.8 \\
\hline 6 & 42.4 & 15.9 & 37.6 & 7.0 & 16.4 & 6.9 & 16.2 & 0.4 & 1.0 \\
\hline 7 & 853.1 & 85.2 & 10.0 & 44.1 & 5.2 & 6.1 & 0.7 & 0.1 & 0.1 \\
\hline 8 & 148.7 & 47.9 & 32.2 & 19.4 & 13.1 & 13.0 & 8.7 & 0.3 & 0.7 \\
\hline 9 & 168.2 & 65.8 & 39.1 & 57.7 & 34.3 & 29.7 & 17.6 & 0.5 & 0.5 \\
\hline 10 & 94.7 & 34.3 & 36.2 & 51.4 & 54.3 & 32.2 & 34.0 & 0.9 & 0.6 \\
\hline mean & $199 \pm 244$ & $48 \pm 27$ & $35 \pm 12$ & $30 \pm 20$ & $24 \pm 20$ & $18 \pm 13$ & $17 \pm 14$ & $0.4 \pm 0.3$ & $0.8 \pm 0.3$ \\
\hline
\end{tabular}

Abbreviations: $\mathrm{GTV}_{\text {prim }}=$ primary gross tumour volume; $\mathrm{GTV}_{\mathrm{FDG}}=$ volume of $\mathrm{GTV}_{\text {prim }}$ with FDG uptake $>50 \% \mathrm{SUV}_{\text {max }}$; $\mathrm{GTV}_{\mathrm{HX} 4}=$ volume of $\mathrm{GTV}_{\text {prim }}$ with a tumour-to-background ratio $>1.4 ; \mathrm{OF}=$ overlapping fraction; $\mathrm{OF}_{\mathrm{FDG}}=$ overlapping volume divided by FDG volume; $\mathrm{OF}_{\mathrm{HX} 4}=$ overlapping volume divided by $\mathrm{HX} 4$ volume . 


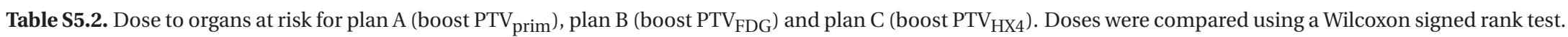

\begin{tabular}{|c|c|c|c|c|c|c|c|c|c|c|c|c|c|c|c|c|c|c|c|c|c|}
\hline \multirow[t]{2}{*}{ Patient } & \multicolumn{3}{|c|}{$\begin{array}{c}\text { Mean lung } \\
\text { dose (Gy) }\end{array}$} & \multicolumn{3}{|c|}{$\begin{array}{c}\text { EQD2 mean lung } \\
\text { dose (Gy) }\end{array}$} & \multicolumn{3}{|c|}{$\begin{array}{c}\text { PRV mediastinal } \\
\text { structures } \mathrm{D}_{0.1}(\mathrm{~Gy})\end{array}$} & \multicolumn{3}{|c|}{$\begin{array}{l}\text { Spinal cord } \\
\mathrm{D}_{0.1}(\mathrm{~Gy})\end{array}$} & \multicolumn{3}{|c|}{$\begin{array}{c}\text { Oesophagus } \\
\mathrm{V}_{36}(\%) \\
\end{array}$} & \multicolumn{3}{|c|}{$\begin{array}{c}\text { Oesophagus } \\
\mathrm{D}_{0.1}(\mathrm{~Gy})\end{array}$} & \multicolumn{3}{|c|}{$\begin{array}{l}\text { Oesophagus } \\
\mathrm{D}_{\text {mean }}(\mathrm{Gy})\end{array}$} \\
\hline & A & B & C & A & B & $\bar{C}$ & A & B & $\mathrm{C}$ & A & B & $\bar{C}$ & A & B & $\bar{C}$ & A & B & $\mathrm{C}$ & A & B & C \\
\hline 1 & 18.4 & 17.9 & 17.8 & 17.9 & 20.0 & 19.1 & 75.9 & 76.3 & 74.1 & 32.2 & 22.9 & 23.3 & 0.3 & 0.0 & 0.0 & 36.9 & 24.6 & 25.0 & 13.2 & 10.5 & 10.0 \\
\hline 2 & 20.8 & 21.1 & 21.3 & 13.4 & 20.1 & 19.8 & 75.2 & 74.0 & 74.4 & 48.5 & 46.9 & 48.7 & 46.3 & 36.0 & 37.6 & 71.7 & 73.2 & 74.1 & 32.9 & 32.9 & 34.0 \\
\hline 3 & 19.5 & 20.2 & 20.4 & 17.8 & 15.2 & 20.1 & 74.8 & 76.1 & 75.4 & 43.8 & 47.5 & 50.0 & 28.1 & 29.5 & 32.9 & 70.9 & 73.0 & 71.8 & 22.0 & 25.5 & 25.9 \\
\hline 4 & 13.8 & 13.9 & 14.0 & 18.5 & 17.7 & 20.0 & 70.4 & 66.8 & 66.2 & 38.7 & 34.7 & 32.9 & 5.1 & 9.7 & 9.6 & 49.7 & 52.2 & 53.6 & 12.6 & 14.0 & 14.0 \\
\hline 5 & 21.3 & 21.3 & 20.8 & 19.0 & 13.3 & 20.2 & 71.9 & 75.0 & 75.2 & 47.1 & 45.5 & 49.8 & 52.3 & 52.7 & 52.0 & 71.9 & 75.4 & 75.1 & 35.7 & 37.7 & 38.2 \\
\hline 6 & 20.5 & 20.6 & 20.9 & 19.4 & 17.3 & 14.6 & 76.1 & 74.0 & 71.6 & 50.9 & 49.1 & 49.1 & 45.2 & 43.2 & 45.3 & 69.5 & 72.7 & 71.2 & 31.8 & 31.9 & 32.8 \\
\hline 7 & 18.5 & 18.4 & 17.7 & 20.0 & 18.0 & 17.6 & 75.4 & 76.0 & 76.1 & 49.1 & 50.2 & 49.4 & 54.2 & 59.0 & 52.7 & 77.5 & 77.4 & 75.9 & 39.8 & 40.3 & 37.0 \\
\hline 8 & 15.6 & 15.5 & 15.5 & 19.7 & 19.4 & 12.9 & 76.0 & 76.0 & 75.3 & 43.4 & 39.4 & 50.4 & 29.9 & 22.4 & 27.9 & 72.1 & 68.7 & 71.6 & 22.1 & 20.2 & 20.8 \\
\hline 9 & 20.2 & 20.6 & 20.3 & 20.1 & 19.2 & 16.8 & 73.5 & 76.3 & 76.0 & 44.1 & 31.5 & 31.2 & 22.3 & 31.6 & 24.3 & 67.9 & 71.1 & 69.9 & 19.5 & 23.0 & 20.2 \\
\hline 10 & 19.1 & 18.9 & 19.2 & 15.1 & 20.0 & 18.5 & 75.7 & 74.7 & 74.3 & 41.0 & 39.0 & 39.2 & 15.3 & 18.2 & 18.6 & 63.9 & 62.2 & 67.9 & 18.8 & 18.8 & 18.9 \\
\hline mean & $19 \pm 2$ & $19 \pm 3$ & $19 \pm 2$ & $18 \pm 2$ & $18 \pm 2$ & $18 \pm 2$ & $74 \pm 2$ & $75 \pm 3$ & $74 \pm 3$ & $44 \pm 6$ & $41 \pm 9$ & $42 \pm 10$ & $30 \pm 19$ & $30 \pm 18$ & $30 \pm 18$ & $65 \pm 12$ & $65 \pm 16$ & $66 \pm 17$ & $25 \pm 10$ & $25 \pm 10$ & $25 \pm 10$ \\
\hline vs plan A (p-value) & - & 0.508 & 0.799 & - & 0.721 & 0.878 & - & 0.878 & 0.386 & - & 0.037 & 0.646 & - & 0.646 & 0.799 & - & 0.575 & 0.169 & - & 0.262 & 0.575 \\
\hline vs plan B (p-value) & 0.508 & - & 0.878 & 0.721 & & 0.646 & 0.878 & - & 0.037 & 0.037 & - & 0.169 & 0.646 & - & 0.859 & 0.575 & - & 0.799 & 0.262 & - & 0.813 \\
\hline vs plan C (p-value) & 0.799 & 0.878 & - & 0.878 & 0.646 & - & 0.386 & 0.037 & - & 0.646 & 0.169 & - & 0.799 & 0.859 & - & 0.169 & 0.799 & - & 0.575 & 0.813 & - \\
\hline
\end{tabular}

Abbreviations: EQD2 = equivalent 2 Gy dose; PRV = planning risk volume; $\mathrm{D}_{0.1}=$ dose received by maximal $0.1 \%$ of the organ; $\mathrm{V}_{36}=$ volume receiving at least $36 \mathrm{~Gy}$; $\mathrm{D}_{\mathrm{mean}}$ is the mean dose. 



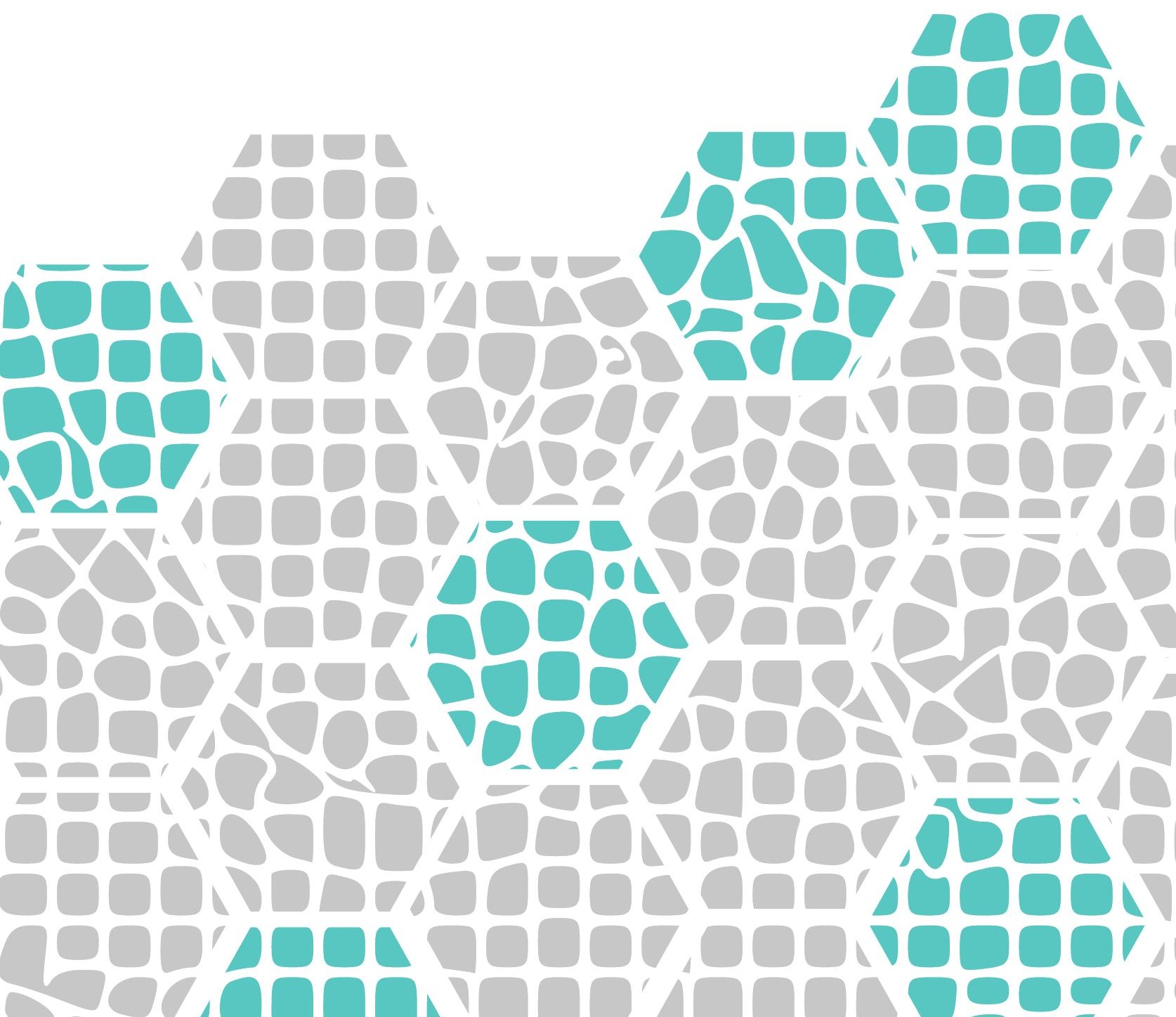




\section{Combining multiparametric imaging characteristics}

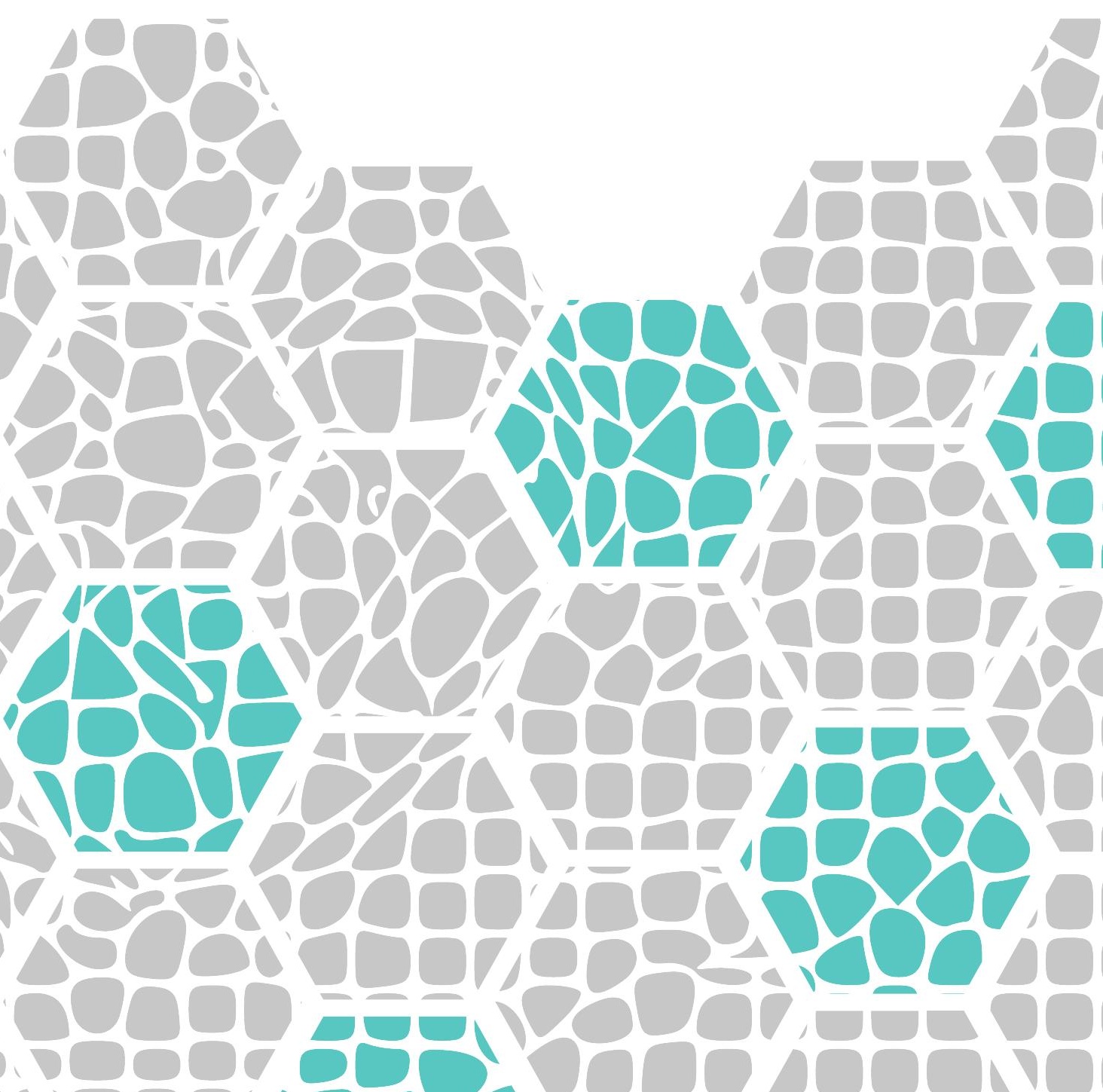




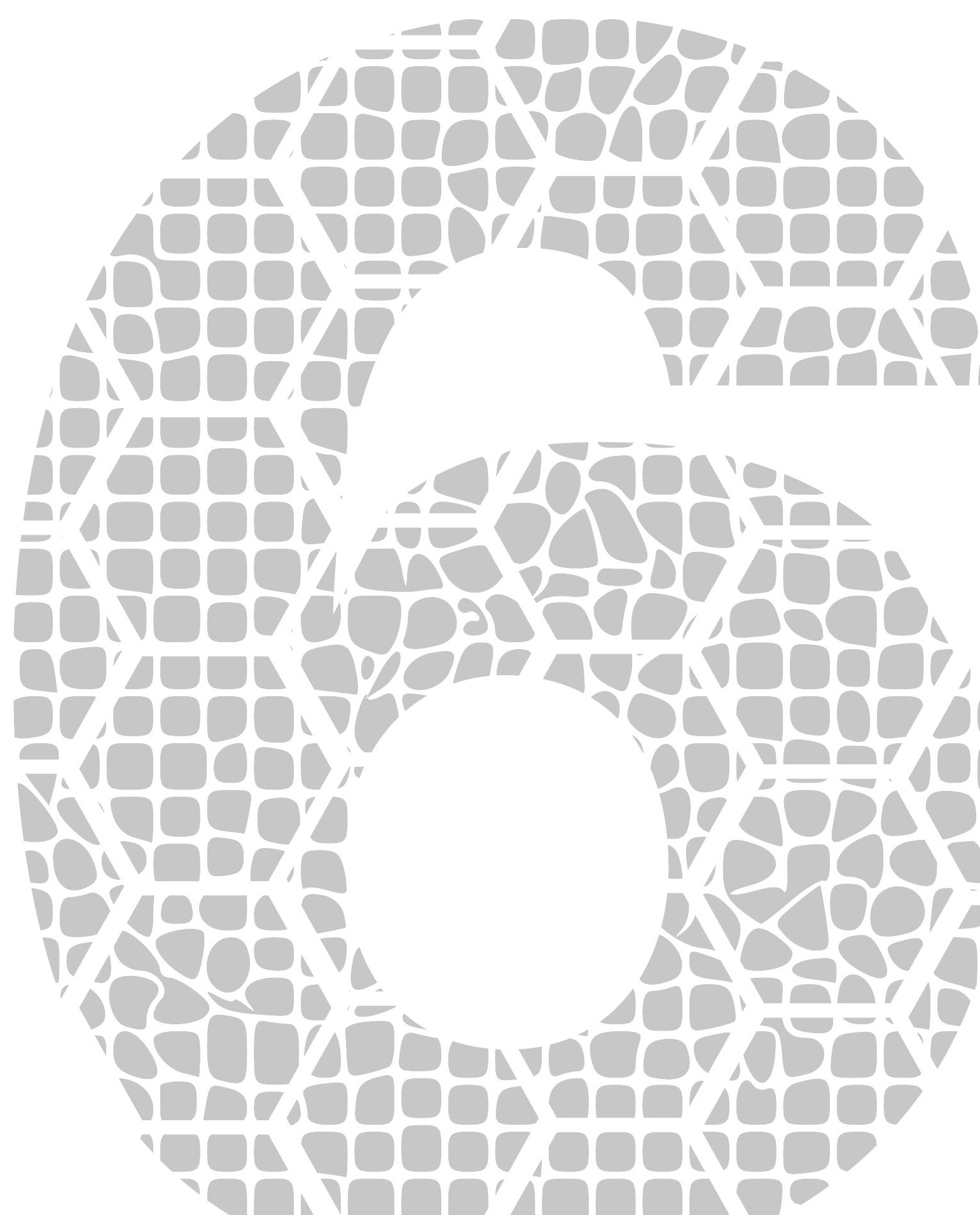




\title{
Predicting tumour hypoxia in non- small cell lung cancer by combining CT, FDG PET and dynamic contrast- enhanced CT
}

\author{
Aniek J.G. Even, Bart Reymen, Matthew D. La Fontaine, Marco Das, \\ Arthur Jochems, Felix M. Mottaghy, José S.A. Belderbos, Dirk De Ruysscher, \\ Philippe Lambin, Wouter van Elmpt
}




\begin{abstract}
Background Most solid tumours contain inadequately oxygenated (i.e. hypoxic) regions, which tend to be more aggressive and treatment resistant. Hypoxia PET allows visualization of hypoxia and may enable treatment adaptation. However, hypoxia PET imaging is expensive, timeconsuming, and not widely available. We aimed to predict hypoxia levels in non-small cell lung cancer (NSCLC) using more easily available imaging modalities: FDG PET/CT and dynamic contrast-enhanced CT (DCE-CT).

Material and methods For 34 NSCLC patients, included in two clinical trials, hypoxia HX4 PET/CT, planning FDG PET/CT, and DCE-CT scans were acquired before radiotherapy. Scans were non-rigidly registered to the planning CT. Tumour blood flow (BF) and blood volume (BV) were calculated by kinetic analysis of DCE-CT images. Within the gross tumour volume, independent clusters, i.e. supervoxels, were created based on FDG PET/CT. For each supervoxel, tumour-to-background ratios (TBR) were calculated (median SUV / aorta $\mathrm{SUV}_{\text {mean }}$ ) for HX4 PET/CT, and supervoxel features (median, SD, entropy) for the other modalities. Two random forest models (cross-validated: ten folds, five repeats) were trained to predict the hypoxia TBR; one based on CT, FDG, BF and BV, and one with only CT and FDG features. Patients were split in a training (trial NCT01024829) and independent test set (trial NCT01210378). For each patient, predicted, and observed hypoxic volumes (TBR > 1.2) were compared.
\end{abstract}

Results Fifteen patients (3291 supervoxels) were used for training and 19 patients (1502 supervoxels) for testing. The model with all features (RMSE training: $0.19 \pm 0.01$, test: 0.27 ) outperformed the model with only CT and FDG PET features (RMSE training: $0.20 \pm 0.01$, test: 0.29 ). All tumours of the test set were correctly classified as normoxic or hypoxic (hypoxic volume $>1 \mathrm{~cm}^{3}$ ) by the best performing model.

Conclusion We created a data-driven methodology to predict hypoxia levels and hypoxia spatial patterns using CT, FDG PET, and DCE-CT features in NSCLC. The model correctly classifies all tumours, and could therefore, aid tumour hypoxia classification and patient stratification. 


\section{Introduction}

Hypoxia is one of the important factors influencing treatment outcome in cancer patients. Most solid tumours contain hypoxic, poorly oxygenated, regions, which are prone to be more radioresistant, chemoresistant, and aggressive. This is due to multiple features such as an altered metabolism, increased resistance to cell death, increased angiogenesis and invasiveness, and tendency to metastasize [1-3].

Although hypoxic tumours are less sensitive to conventional treatments, they might benefit from specially designed targeted treatments such as hypoxia activated prodrugs or the inhibition of molecular targets in hypoxic cells [1-4]. Other approaches include increasing the radiotherapy dose in the hypoxic areas to overcome radioresistance $[5,6]$, or adding radiosensitizers to radiotherapy treatment [7]. To select patients that will benefit the most from these targeted strategies, in clinical routine, as well as in clinical trials, there is a need for non-invasive techniques that can reliably detect hypoxia. In most clinical trials, patients are not stratified by hypoxia status and therefore these trials most likely do not demonstrate the full potential of hypoxia targeting strategies.

The most studied non-invasive techniques to visualize hypoxia make use of PET tracers based on 2-nitroimidazoles labelled with Fluorine-18, for example ${ }^{18} \mathrm{~F}$-MISO, ${ }^{18} \mathrm{~F}$-FAZA and ${ }^{18} \mathrm{~F}-\mathrm{HX} 4$ [8]. In the absence of oxygen, the reduction of nitroimidazoles cannot be reversed and the reactive product gets trapped in the hypoxic cell. Other studied imaging techniques make use of more indirect ways of assessing hypoxia. Perfusion and diffusion based approaches, for example, have been suggested as surrogates for hypoxia, since the delivery of oxygen to tumours is regulated by blood supply. CT-based perfusion techniques, dynamic contrast-enhanced CT (DCE-CT), as well as MRI-based modalities, DCE-MRI or diffusion weighted MRI (DW-MRI), have been proposed. However, hypoxia is not only influenced by perfusion; perfusion markers cannot visualize the ability of blood to carry and release oxygen or the oxygen consumption rate of cells. ${ }^{18} \mathrm{~F}-\mathrm{FDG}$ PET has been suggested as another indirect marker of hypoxia [9]. Hypoxic cells have to resort to the less efficient glycolysis for their energy production resulting in an upregulated metabolism that can be visualized with the commonly used FDG PET tracer. Unfortunately, cancer cells tend to predominately rely on glycolysis even under well oxygenated conditions, hindering the distinction between hypoxic and non-hypoxic tumour cells [10].

PET tracers based on 2-nitroimidazoles are the most studied and most direct way of in vivo visualizing of hypoxia. However, so far, these PET images have only been used in research or clinical trial setting, as they are not widely available, expensive, and timeconsuming. In this study we investigated the possibility of replacing a 2-nitroimidazole PET tracer by alternative indirect hypoxia markers that are more accessible. We aimed 
to predict hypoxia levels in patients with non-small cell lung cancer (NSCLC) tumours, assessed with HX4 PET, by using FDG PET/CT and dynamic contrast-enhanced CT imaging.

\section{Materials and Methods}

\section{Patient selection}

Stage IB-IV NSCLC patients were included in two prospective clinical trials (PETboost trial: NCT01024829 [11]; Nitroglycerin trial: NCT01210378). The pretreatment imaging protocol was identical for both clinical studies, including a planning FDG PET/CT, hypoxia HX4 PET/CT, and dynamic contrast-enhanced CT (DCE-CT). The HX4 PET/CT and DCE-CT scans were preferably scheduled on the same day; FDG and HX4 PET/CT scans were always planned on different days. The three scans were planned within the same week before radiotherapy for all except one patient. The time between scans ranged from 1 to 11 days. All patients gave written informed consent and both studies were approved by the Medical Ethics Review Committee. For our analysis, we selected all patients with images of all treatment modalities available.

\section{Image acquisition and preprocessing}

All scans were performed in treatment position on a flat table top with the same arm and knee supports used during radiotherapy treatment.

\section{Planning FDG PET/CT}

A 4D respiratory gated FDG PET/CT was acquired one hour after injection of approximately $197 \pm 43 \mathrm{MBq}$ FDG tracer, according to the NEDPAS protocol [12], on a Siemens Biograph 40 PET/CT scanner (Siemens Healthcare, Erlangen, Germany). The midventilation phase of the 4D CT scan was used for attenuation correction. PET scans were also corrected for scatter and random coincidences. The PET images were reconstructed in voxels of 4 by $4 \mathrm{~mm}$ in-plane with a slice thickness of $3 \mathrm{~mm}$ using an OSEM or PSF algorithm. The voxels of the CT were 1 by 1 by $3 \mathrm{~mm}$. The gross tumour volume (GTV) was delineated on the fused PET/CT by an experienced radiation oncologist. Finally, standardized uptake values (SUV) were calculated by correcting the measured uptake for body weight, injected dose, and decay.

\section{Hypoxia HX4 PET/CT}

A 2-nitroimidazole-based PET tracer, $\left[{ }^{18} \mathrm{~F}\right] \mathrm{HX} 4$ (3- $\left[{ }^{18} \mathrm{~F}\right]$ fluoro-2-(4-((2-nitro- $1 \mathrm{H}-$ imidazol-1-yl)methyl)-1H-1,2,3-triazol-1-yl)propan-1-ol), from here on referred to as 
HX4 [13-15], was used to acquire hypoxia PET images. The patients were scanned four hours postinjection of approximately $417 \pm 77 \mathrm{MBq}$ of HX4 on a Gemini TF64 PET/CT scanner (Philips, the Netherlands). Scans were corrected for attenuation using a lowdose CT scan, and corrected for scatter and random coincidences. The voxels of the reconstructed PET images (BLOB-OS-TF with 3 iterations and 33 subsets) had an inplane size of 4 by $4 \mathrm{~mm}$ and slice thickness of $4 \mathrm{~mm}$. The PET uptake in the aortic arch was measured to calculate the tumour-to-background ratio (TBR), which was defined as the SUV in the tumour divided by the $\mathrm{SUV}_{\text {mean }}$ in the aortic arch.

\section{Dynamic contrast-enhanced CT}

For the DCE-CT images, patients were injected with 60 or $65 \mathrm{ml}$ of an iodine-based contrast material (Iopromide 300, Bayer Healthcare, Berlin, Germany), followed by a saline chaser of $30 \mathrm{ml}$ or $40 \mathrm{ml}$. Both at a flow rate of $7 \mathrm{ml} / \mathrm{s}$. The patients were scanned on a second or third generation dual source scanner (SOMATOM Definition Flash or SOMATOM Force, Siemens Healthcare, Erlangen, Germany). Every 1.5 second $80 \mathrm{kVp}$ CT scans of primary tumour were acquired for 33 consecutive frames. Patients were asked to hold their breath in expiration breath-hold as long as possible and otherwise continue shallow breathing. Scans were reconstructed with a B20f filter with a slice thickness of $5 \mathrm{~mm}$. All 33 frames were aligned to the first slice using a deformable fluid-based registration algorithm (syngo MMWP, version VE40A; Siemens).

Next, kinetic analysis was performed on the DCE-CT scans, using a deconvolution approach (adiabatic approximation to the tissue homogeneity model; ATH) [16, 17]. The images were smoothed with a median 5 filter and resampled to a $256 \times 256$ grid. A region of interest was delineated in the aortic arch to determine the arterial input function. Finally, parametric maps were calculated for tumour blood flow (BF; $\mathrm{ml} / 100 \mathrm{~g} / \mathrm{min}$ ) and blood volume (BV; $\mathrm{ml} / 100 \mathrm{~g})$.

\section{Image registration and supervoxels}

The images of all modalities were mapped onto the planning CT scan using a rigid alignment followed by a non-rigid registration using the open source software Elastix $[18,19]$. Voxels were resampled to the in-plane dimensions of the CT to create isotropic voxels. Next, homogeneous patches, preserving local image boundaries, were created by grouping voxels based on their CT Hounsfield units and FDG SUV, see Figure 6.1. These clusters of voxels, i.e. supervoxels, were introduced to reduce noise, reduce the amount of data, and to minimize the effect of registration uncertainties. Supervoxels were generated using an adapted k-means clustering algorithm called Simple Linear Iterative Clustering (SLIC [20]). This freely available algorithm was tuned to create supervoxels with a size of 400 voxels (typically $0.4 \mathrm{~cm}^{3}$ ) and compactness of 20 . For 


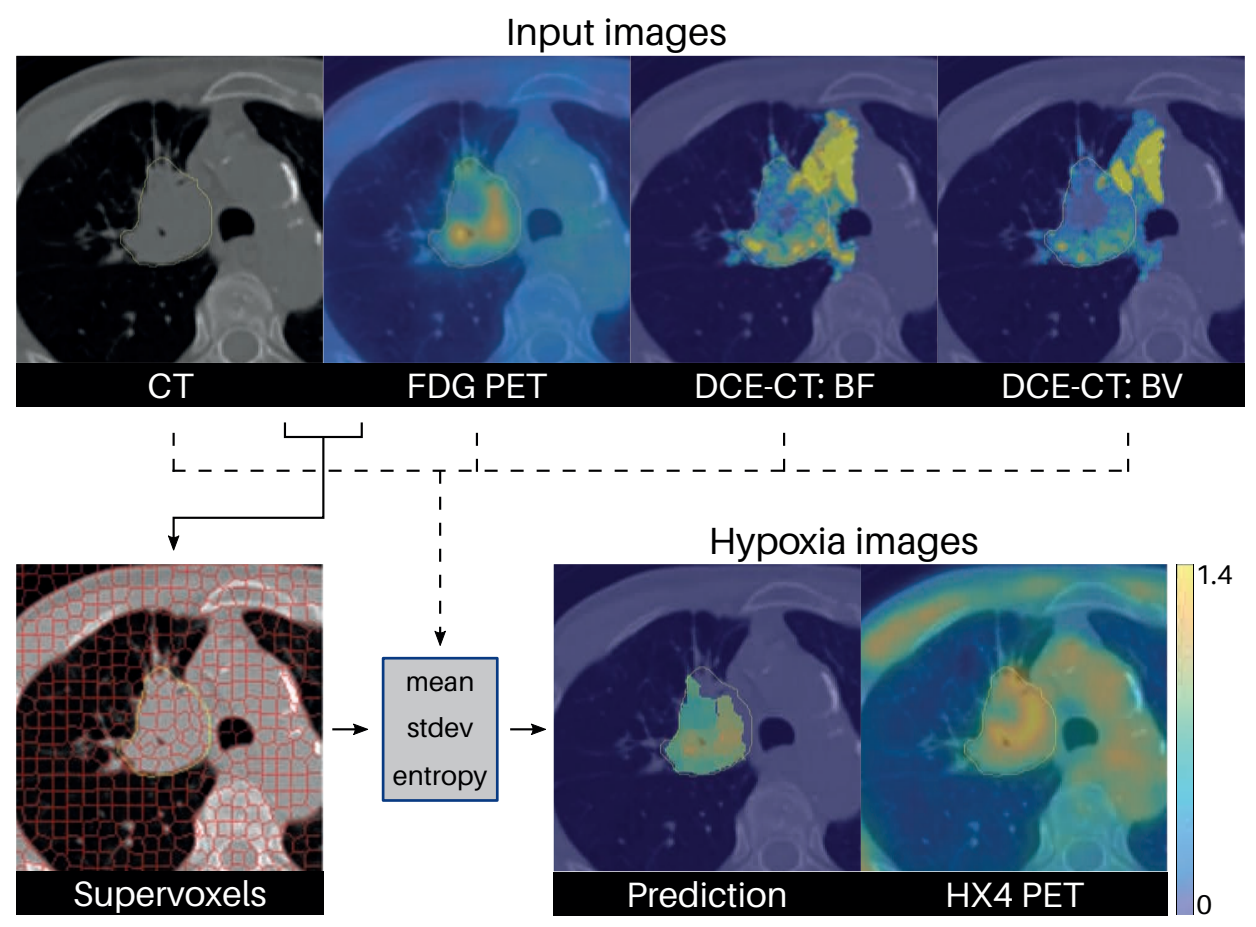

Figure 6.1. An example patient from the test set with the different imaging modalities, the supervoxel preprocessing, and hypoxia images. On the top row, the images that are used as model input: CT, FDG PET, DCE-CT blood flow and blood volume, overlaid on the planning CT. Clustering is performed on the CT and FDG PET data to create supervoxels (solid line). For each supervoxel, the mean, standard deviation and entropy are calculated for each input imaging modality (dashed line). These features are combined in a random forest model to predict hypoxia TBR for every supervoxel. The missing values at the top of the GTV (the contoured structure) in the prediction image are supervoxels with high DCE-CT residuals (e.g. motion) and therefore excluded from analysis.

further analysis we included all supervoxels with at least $90 \%$ of their voxels in the GTV. Supervoxels with high residuals in the DCE-CT kinetic analysis (> $100 \times 33$ (number of DCE-CT frames)) were excluded from the analysis.

For every supervoxel, the median, standard deviation, and entropy of all voxels in the supervoxels were calculated for the CT (HU), FDG PET (SUV), BF ( $\mathrm{ml} / 100 \mathrm{~g} / \mathrm{min}$ ), and BV (ml/100 g). In addition the median HX4 TBR was determined in every supervoxel. 


\section{Building a random forest}

Random forest models were trained in $\mathrm{R}$ (version 3.3.2) to predict hypoxia TBR inside a supervoxel based on CT, FDG PET, BF and BV features. For this purpose, patients were split in a training set (trial NCT01024829) and independent test set (trial NCT01210378). All supervoxels of the tumours in the training set were grouped, likewise for the supervoxels in the test set. The numbers of trees in the random forest were fixed to 500. Cross validation of ten folds and five repeats was applied to tune one model parameter, i.e. number of variables randomly sampled at each split, and to evaluate the performance of the final model. Two models were trained: one containing all features of the CT, FDG PET, BF and BV, and one with only the CT and FDG PET features. Finally, a multivariable linear regression model was trained to serve as benchmark for the random forest models.

\section{Predicting tumour hypoxic volume}

The predicted hypoxia TBR values for all supervoxels for each patient in the test set were used to determine the hypoxic volume (HV). First, the outputs of the random forest models were calibrated by refitting the prediction of the training set with a linear regression and applying the fit to the prediction of the supervoxels in the test set. Next, all supervoxels with a predicted TBR $>1.2$ were defined as hypoxic and summed for the patients in the test set. We chose a threshold of 1.2 in the absence of a standardized threshold. Predicted hypoxic volumes were compared to the observed hypoxic volumes based on the HX4 PET/CT scans. Tumours with a hypoxic volume larger than $1 \mathrm{~cm}^{3}$ were classified as hypoxic. The $1 \mathrm{~cm}^{3}$ threshold was introduced to have a robust metric that takes into account the imaging noise and limited resolution of the imaging modalities.

\section{Evaluating supervoxels}

One extra random forest was trained to quantify the influence of the supervoxel oversegmentation on model performance and to evaluate the need for this preprocessing step. For this, no supervoxels were calculated and the FDG PET and DCE-CT parameter maps were rescaled to the planning CT scan resolution. Voxels with high DCECT residuals were again excluded; all other voxels were used to train and validate the model. 
Table 6.1. Patient and tumour characteristics for all patients together, for the training patients, and the test patients.

\begin{tabular}{llrrr}
\hline & & Total & Training & Test \\
\hline Patients & & 34 & 15 & 19 \\
\hline \multirow{2}{*}{ Gender } & Male & 25 & 12 & 13 \\
& Female & 9 & 3 & 6 \\
\hline \multirow{2}{*}{ GTV } & Median volume $\left(\mathrm{cm}^{3}\right)$ & 49 & 65 & 42 \\
& Volume interquartile range $\left(\mathrm{cm}^{3}\right)$ & $25-91$ & $41-122$ & $10-74$ \\
& Median number of supervoxels & 92 & 117 & 66 \\
& Supervoxel interquartile range & $41-174$ & $71-234$ & $12-143$ \\
\hline \multirow{2}{*}{ TNM (T) } & T2 & 12 & 6 & 6 \\
& T3 & 12 & 4 & 8 \\
& T4 & 10 & 5 & 5 \\
\hline \multirow{2}{*}{ TNM (N) } & N0 & 3 & 1 & 2 \\
& N1 & 2 & 1 & 1 \\
& N2 & 19 & 9 & 10 \\
& N3 & 10 & 4 & 6 \\
\hline \multirow{2}{*}{ TNM (M) } & M0 & 28 & 15 & 13 \\
& M1 & 6 & 0 & 6 \\
\hline Pathology & Adenocarcinoma & 13 & 6 & 7 \\
& Squamous cell carcinoma & 11 & 6 & 5 \\
& Large-cell carcinoma & 6 & 2 & 4 \\
& Not otherwise specified & 4 & 1 & 3 \\
\hline
\end{tabular}

\section{Results}

In total, we included 34 NSCLC patients (age 65 years \pm 8 years) who all received an FDG and an HX4 PET/CT as well as a DCE-CT scan. The median GTV was $49 \mathrm{~cm}^{3}$ (interquartile range (IQR): $25-91 \mathrm{~cm}^{3}$ ). More patient and tumour characteristics are provided in Table 6.1. The gross tumour volumes were subdivided in a median 92 (IQR: 41-174) supervoxels per patient. The training set contained 15 patients, with a total of 3291 supervoxels, while the test cohort consisted of 19 patients with a total of 1502 supervoxels, see Table 6.1. The supervoxels contained on average 405 voxels \pm 76 voxels $\left(0.4 \mathrm{~cm}^{3} \pm 0.1 \mathrm{~cm}^{3}\right)$. An example patient, with the different imaging modalities and the supervoxel segmentation is shown in Figure 6.1.

The supervoxel approach retains spatial information, while improving the signalto-noise ratio. From the predicted TBR values for each supervoxel, hypoxia parameter maps can be reconstructed for a tumour. In Figure 6.1 such a hypoxia map is shown for the best performing random forest model using all imaging features. The predicted hypoxia map shows a visually similar pattern compared to the hypoxia PET/CT scan. 


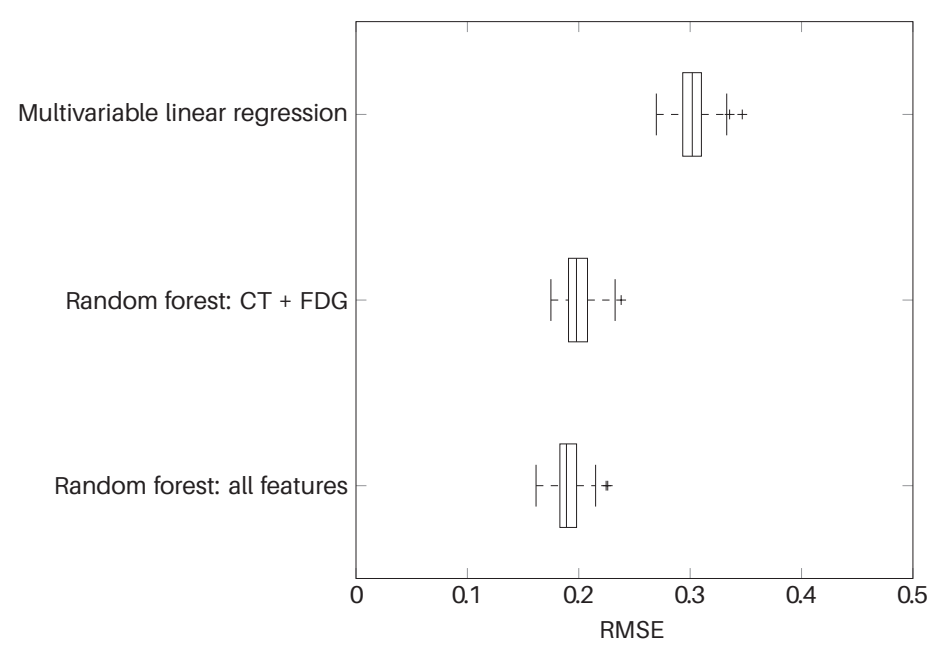

Figure 6.2. Boxplots comparing the root mean square error (RMSE) of the three different models. The models are ranked from worst to best performance on the training set based on crossvalidation (10 folds, five repeats).

The random forest model with all features $(n=12)$ gave the best performance with a root-mean-square error (RMSE) in hypoxia TBR prediction of $0.19 \pm 0.01$ for the training set, and 0.27 for the test set. The random forest with only CT and FDG features $(n=6)$ had a slightly worse performance with a RMSE of $0.20 \pm 0.01$ for training and 0.29 for the test set. The restricted random forest model still outperformed the multivariable linear regression with all features (training RMSE: $0.30 \pm 0.02$; test RMSE: 0.43). In Figure 6.2 the RMSE of the models are compared. The random forest directly trained on voxel values, i.e. without using supervoxel preprocessing, had a higher RMSE compared to both supervoxel random forest models with $0.26 \pm 0.00$ on the training data and 0.30 on validation.

The hypoxic areas based on the HX4 PET measurements had a median volume of $1.4 \mathrm{~cm}^{3}$ (IQR: $0-10.6 \mathrm{~cm}^{3}$ ) for the 18 patients in the test set. For one of the patients, all supervoxels had a high DCE-CT residual and were excluded from the analysis. The measured hypoxic volumes and predicted volumes had an absolute median difference of $1.1 \mathrm{~cm}^{3}$ (IQR: $0.1-4.9 \mathrm{~cm}^{3}$ ) for the random forest with all features, $0.8 \mathrm{~cm}^{3}$ (IQR: $0.0-6.6 \mathrm{~cm}^{3}$ ) for the restricted random forest model and $1.7 \mathrm{~cm}^{3}$ (IQR: $0.3-11.8 \mathrm{~cm}^{3}$ ) for the multivariable linear regression model. All models underestimated the hypoxic volumes for tumours with large hypoxic volumes, see Figure 6.3.

All tumours in the test set were correctly classified as hypoxic (hypoxic volume $>1 \mathrm{~cm}^{3}$ ) or not-hypoxic by the random forest with features of all image modalities. Both the random forest with CT and FDG features, and the multivariable linear 


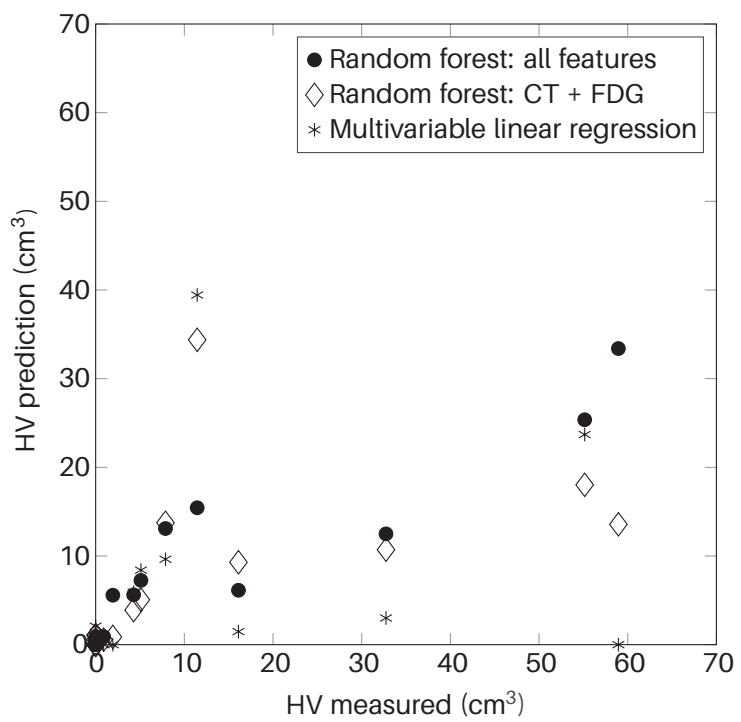

Figure 6.3. Hypoxic volumes (HV) for the patients in the test set: measured HV versus predicted $\mathrm{HV}$ for the two random forest models and multivariable linear regression.

regression model misclassified 3 out of 18 patients, of which two patients were misclassified by both, Figure 6.3.

\section{Discussion}

To our knowledge, the present study is the first data-driven approach to predict baseline hypoxia levels and to create a virtual hypoxia PET image for non-small cell lung cancer patients. Other published approaches to simulate tumour oxygenation have been fully based on mathematical models [21,22], or a combination of a mathematical model and anatomical input, using for example histology [22] or MRI [23]. None of the published methods are solely based on patient specific non-invasive imaging.

The best performance was achieved by training a random forest combining an indirect hypoxia marker linked to perfusion (DCE-CT), a marker related to tumour metabolism (FDG PET), and CT image features. The random forest outperformed a multivariable linear regression using the same features. We managed to validate our random forest in an independent test set of patients of a second clinical trial.

The introduction of supervoxels, instead of creating voxel-based models, tremendously decreased the memory and calculation costs. More importantly, the supervoxel model, with all features, outperformed the voxel-based approach, with a training 
RMSE difference of 0.19 versus 0.26 , and test difference of 0.27 versus 0.30 , respectively. The supervoxel approach provides the additional benefit of being able to calculate multiple features per supervoxel, such as the median, standard deviation, and entropy we investigated. We decided to limit our research to these three commonly used features and did not study other metrics due to the limited number of patients. Other features could potentially further optimize the models.

The random forest model with features of all image modalities outperformed the model with only CT and FDG PET features. However, if DCE-CT imaging is not available, the restricted model could still provide valuable information because the observed performance difference was minimal. FDG PET appears to be the strongest indicator of hypoxia in NSCLC tumours and the most important predictor in our models. Hypoxia levels increase with increasing FDG PET SUV nonlinearly and plateau around a SUV of nine; see Supplementary Figure S6.1 for the individual contributions of all features to the best performing random forest model. Interestingly, voxelwise comparison studies between FDG and hypoxia PET have provided contradicting results regarding their correlation [9, 24]. All other features, show a less pronounced effect on the predicted hypoxia levels and their contributions have larger variations (Supplementary Figure S6.1). The first four most important parameters are based on either FDG PET or CT, and do not include any DCE-CT features. This, in combination with the limited increase in model performance between the random forest based on FDG PET/CT or all image features, questions the potential of perfusion imaging as a hypoxia marker in NSCLC. The relationship between DCE-CT and hypoxia PET has not been studied intensively. On a tumour level, negative correlations between perfusion parameters and hypoxia have been shown [25]. Between immunohistochemical hypoxia markers and DCE-CT parameters also moderate negative correlations have been found [26]. One hypothesis that could explain the limited influence of DCE-CT on our hypoxia prediction is the difference in signal generation between hypoxia PET and DCE-CT imaging. Hypoxia PET tracers are trapped at the cellular level and only require a relatively small amount of positron emitters to generate a signal, while DCE-CT analysis compares differences in contrast uptake over time and averages the signal within every voxel.

The prediction of the hypoxic volumes is correct for NSCLC tumours with small hypoxic regions, however, the larger hypoxic volumes were all underestimated. One possible explanation would be that the training set does not represent the test set well enough. We decided to separate the data of the two clinical trials in a training and independent test set. The inclusion criteria of the trials are however different, and therefore also the characteristics of the tumours. In our training data set (NCT01024829, a dose escalation trial based on FDG PET/CT imaging), only patients were included with a minimal tumour diameter of $4 \mathrm{~cm}$, a pretreatment FDG SUV max 
of 5.0, and no distant metastases. While in the test cohort (NCT01210378, standard (chemo)radiotherapy with the addition of a vasodilating drug), inclusion criteria were less rigid and almost all stage IB-IV NSCLC with curative intent were eligible, typically including also smaller size lesions, see also Table 6.1. It is possible that not all characteristics of the tumours in the test set are provided in the training data. We decided to keep the two clinical trials separate and to train the models on the data set with the most supervoxels. Mixing both datasets would most likely yield a better result, however, the test and training set will no longer be independent and it is more difficult to determine the generalizability of the model. Although it is rather unique to have multimodality pretreatment imaging of this many patients, more as well as more diverse patients are needed to further optimize the model. Furthermore, the generalizability of the model should be tested in external data sets, possibly by including also other 2-nitroimidazole PET tracers working with the same mechanism such as FAZA or FMISO.

The extended random forest model correctly classified all tumours as hypoxic or normoxic. Such a model could be applied to select NSCLC patients for hypoxia targeting therapies (e.g. hypoxia targeted prodrugs), if hypoxia PET imaging is not available. Preferably, the model would be based solely on FDG PET and CT as these images are already available in clinical routine and the DCE-CT scan adds an extra radiation and time burden to the patient. The random forest model without the DCE-CT features, however, misclassified 3 out of 18 patients. The performance of this restricted model is not sufficient enough for patient stratification and should be further improved before implementing in clinical practise. Our described modelling approach provides not only information on the total hypoxic volume of the tumour, but also gives spatial information which could be used to further personalize treatment. The described models, however, underestimate, the larger hypoxic volumes. This should be improved in further research, possibly by using more (diverse) patients to train the models on.

In conclusion, we created a methodology to predict hypoxia levels and hypoxia spatial patterns in non-small cell lung cancer using multiparametric imaging. This data-driven strategy can aid tumour hypoxia classification and patient stratification.

\section{Acknowledgements}

Authors acknowledge financial support from ERC advanced grant (ERC-ADG-2015, $\mathrm{n}^{\circ} 694812$ - Hypoximmuno) This research is also supported by the Dutch technology Foundation STW (grant $n^{\circ} 10696$ DuCAT \& $n^{\circ}$ P14-19 Radiomics STRaTegy), which is the applied science division of NWO, and the Technology Programme of the Ministry of Economic Affairs. Authors also acknowledge financial support from the EU 7th 
framework program (ARTFORCE - $\mathrm{n}^{\circ}$ 257144, REQUITE - $\mathrm{n}^{\circ}$ 601826), SME Phase 2 (EU proposal 673780 - RAIL), EUROSTARS (DART), the European Program H20202015-17 (BD2Decide - PHC30-689715 and ImmunoSABR - $\mathrm{n}^{\circ}$ 733008), Interreg VA Euregio Meuse-Rhine ("Euradiomics"), Kankeronderzoekfonds Limburg from the Health Foundation Limburg and the Dutch Cancer Society. We thank Robert Jeraj and the IGT group at the University of Madison, Wisconsin for providing the DCE-CT modelling. 


\section{References}

1. Wilson, W. R. \& Hay, M. P. Targeting hypoxia in cancer therapy. Nat Rev Cancer 11, 393-410 (2011).

2. Wouters, B. G., van den Beucken, T., Magagnin, M. G., Lambin, P. \& Koumenis, C. Targeting hypoxia tolerance in cancer. Drug Resist Updat 7, 25-40 (2004).

3. Wouters, B. G., Koritzinsky, M., Chiu, R. K., Theys, J., Buijsen, J. \& Lambin, P. Modulation of cell death in the tumor microenvironment. Semin Radiat Oncol 13, 31-41 (2003).

4. Peeters, S. G. J. A., Zegers, C. M. L., Biemans, R., et al. TH-302 in combination with radiotherapy enhances the therapeutic outcome and is associated with pretreatment $\left[{ }^{18}\right.$ F]HX4 hypoxia PET imaging. Clin Cancer Res 21, 2984-92 (2015).

5. Di Perri, D., Lee, J. A., Bol, A., et al. Evolution of $\left[{ }^{18} \mathrm{~F}\right]$ fluorodeoxyglucose and $\left[{ }^{18} \mathrm{~F}\right]$ fluoroazomycin arabinoside PET uptake distributions in lung tumours during radiation therapy. Acta Oncol 56, 516-24 (2017).

6. Even, A. J. G., van der Stoep, J., Zegers, C. M. L., et al. PET-based dose painting in non-small cell lung cancer: Comparing uniform dose escalation with boosting hypoxic and metabolically active sub-volumes. Radiother Oncol 116, 281-6 (2015).

7. Begg, A. C., Stewart, F. A. \& Vens, C. Strategies to improve radiotherapy with targeted drugs. Nat Rev Cancer 11, 239-53 (2011).

8. Peeters, S. G. J. A., Zegers, C. M. L., Lieuwes, N. G., et al. A comparative study of the hypoxia PET tracers $\left[{ }^{18} \mathrm{~F}\right]-\mathrm{HX} 4,\left[{ }^{18} \mathrm{~F}\right]-\mathrm{FAZA}$, and $\left[{ }^{18} \mathrm{~F}\right]$-FMISO in a preclinical tumor model. Int J Radiat Oncol Biol Phys 91, 351-9 (2015).

9. Zegers, C. M. L., van Elmpt, W., Reymen, B., et al. In vivo quantification of hypoxic and metabolic status of NSCLC tumors using $\left[{ }^{18} \mathrm{~F}\right] \mathrm{HX} 4$ and $\left[{ }^{18} \mathrm{~F}\right] \mathrm{FDG}$-PET/CT imaging. Clin Cancer Res 20, 6389-97 (2014).

10. Horsman, M. R., Mortensen, L. S., Petersen, J. B., Busk, M. \& Overgaard, J. Imaging hypoxia to improve radiotherapy outcome. Nat Rev Clin Oncol 9, 674-87 (2012).

11. Van Elmpt, W., De Ruysscher, D., van der Salm, A., et al. The PET-boost randomised phase II dose-escalation trial in non-small cell lung cancer. Radiother Oncol 104, 67-71 (2012).

12. Boellaard, R., Oyen, W. J. G., Hoekstra, C. J., et al. The Netherlands protocol for standardisation and quantification of FDG whole body PET studies in multicentre trials. Eur J Nucl Med Mol Imaging 35, 2320-33 (2008). 
13. Dubois, L. J., Lieuwes, N. G., Janssen, M. H. M., et al. Preclinical evaluation and validation of $\left[{ }^{18} \mathrm{~F}\right] \mathrm{HX} 4$, a promising hypoxia marker for PET imaging. Proc Natl Acad Sci USA 108, 14620-5 (2011).

14. Van Loon, J., Janssen, M. H. M., Öllers, M., et al. PET imaging of hypoxia using $\left[{ }^{18}\right.$ F]HX4: a phase I trial. Eur J Nucl Med Mol Imaging 37, 1663-8 (2010).

15. Zegers, C. M. L., van Elmpt, W., Hoebers, F. J. P., et al. Imaging of tumour hypoxia and metabolism in patients with head and neck squamous cell carcinoma. Acta Oncol 54, 1378-84 (2015).

16. La Fontaine, M. D., McDaniel, L. S., Kubicek, L. N., Chappell, R. J., Forrest, L. J. \& Jeraj, R. Patient characteristics influencing the variability of distributed parameter-based models in DCE-CT kinetic analysis. Vet Comp Oncol 15, 105-17 (2017).

17. St Lawrence, K. S. \& Lee, T. Y. An adiabatic approximation to the tissue homogeneity model for water exchange in the brain: I. Theoretical derivation. J Cereb Blood Flow Metab 18, 1365-77 (1998).

18. Staring, M., Bakker, M. E., Stolk, J., Shamonin, D. P., Reiber, J. H. C. \& Stoel, B. C. Towards local progression estimation of pulmonary emphysema using CT. Med Phys 41, 021905 (2014).

19. Klein, S., Staring, M., Murphy, K., Viergever, M. A. \& Pluim, J. P. W. Elastix: a toolbox for intensity-based medical image registration. IEEE Trans Med Imaging 29, 196205 (2010).

20. Achanta, R., Shaji, A., Smith, K., Lucchi, A., Fua, P. \& Susstrunk, S. SLIC superpixels compared to state-of-the-art superpixel methods. IEEE Trans Pattern Anal Mach Intell 34, 2274-82 (2012).

21. Kelly, C. J. \& Brady, M. A model to simulate tumour oxygenation and dynamic $\left[{ }^{18} \mathrm{~F}\right]$-Fmiso PET data. Phys Med Biol 51, 5859-73 (2006).

22. Grimes, D. R., Kannan, P., Warren, D. R., et al. Estimating oxygen distribution from vasculature in three-dimensional tumour tissue. J R Soc Interface 13, 20160070 (2016).

23. Gu, S., Chakraborty, G., Champley, K., et al. Applying a patient-specific biomathematical model of glioma growth to develop virtual $\left[{ }^{18} \mathrm{~F}\right]$-FMISO-PET images. Math Med Biol 29, 31-48 (2012).

24. Kerner, G. S. M. A., Bollineni, V. R., Hiltermann, T. J. N., et al. An exploratory study of volumetric analysis for assessing tumor response with ${ }^{18} \mathrm{~F}$-FAZA PET/CT in patients with advanced non-small-cell lung cancer (NSCLC). EJNMMI Res 6, 33 (2016). 
25. Van Elmpt, W., Zegers, C. M. L., Reymen, B., et al. Multiparametric imaging of patient and tumour heterogeneity in non-small-cell lung cancer: quantification of tumour hypoxia, metabolism and perfusion. Eur J Nucl Med Mol Imaging 43, 240-8 (2016).

26. Mandeville, H. C., Ng, Q. S., Daley, F. M., et al. Operable non-small cell lung cancer: correlation of volumetric helical dynamic contrast-enhanced CT parameters with immunohistochemical markers of tumor hypoxia. Radiology 264, 581-9 (2012). 


\section{Supplementary data}

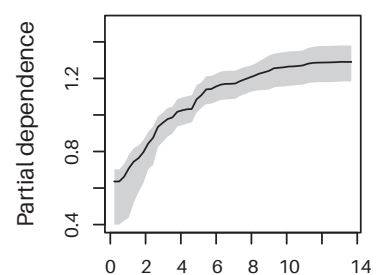

FDG PET (SUV)
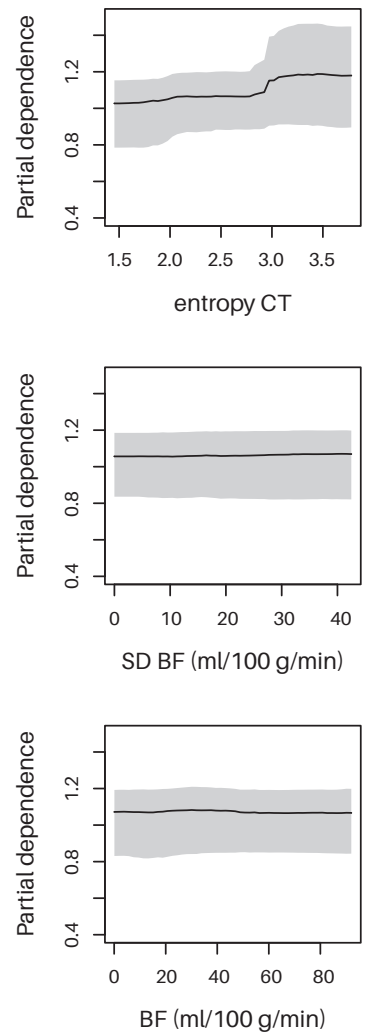
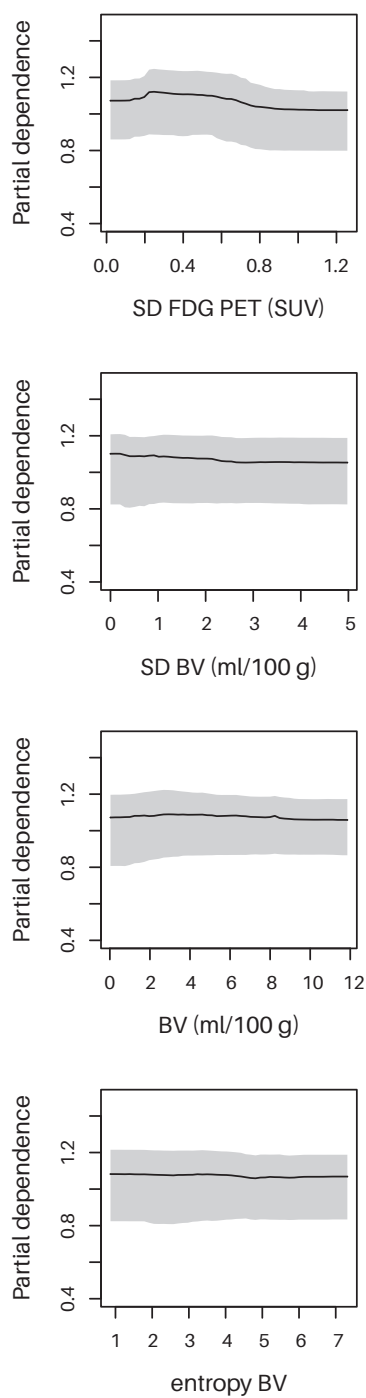
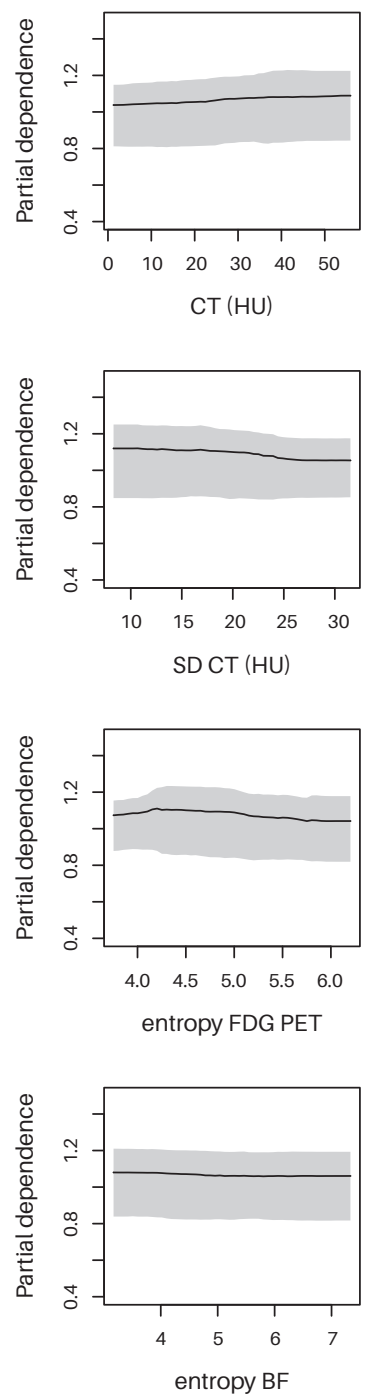

Figure S6.1. Partial dependence plots of all features in the best performing random forest model. The plots indicate the partial relationship between the individual features and the modelled outcome, while keeping all other features constant. The features are ordered by importance, in reading direction. The grey area depicts the interquartile range and the black line the median partial dependence. 

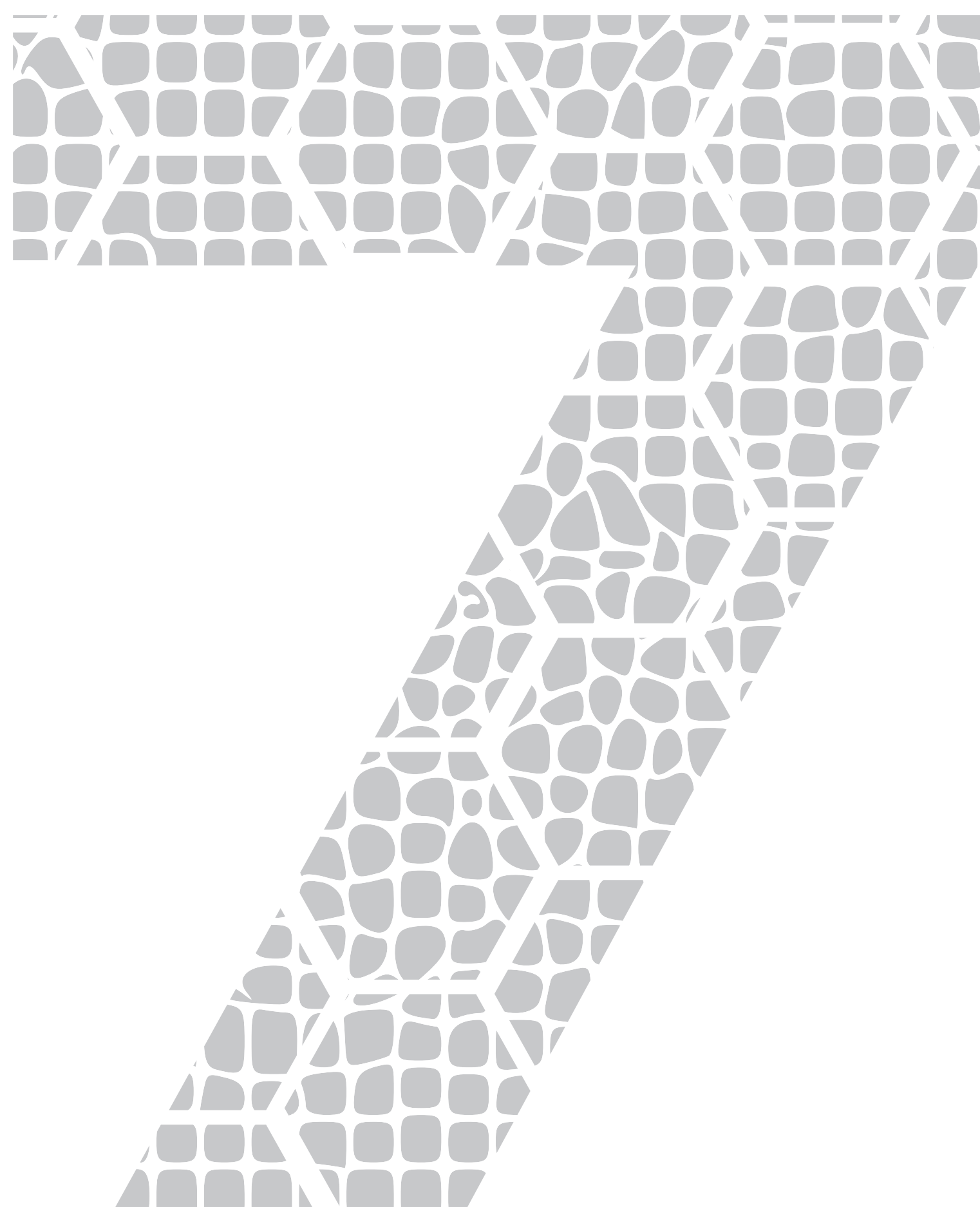


\section{Clustering of multiparametric functional imaging to identify high- risk subvolumes in non-small cell lung cancer}

Aniek J.G. Even, Bart Reymen, Matthew D. La Fontaine, Marco Das, Felix M. Mottaghy, José S.A. Belderbos, Dirk De Ruysscher, Philippe Lambin, Wouter van Elmpt 


\begin{abstract}
Background and purpose We aimed to identify tumour subregions with characteristic phenotypes based on pretreatment multiparametric functional imaging and to correlate these subregions to treatment outcome. The subregions were created using imaging of metabolic activity (FDG PET/CT), hypoxia (HX4 PET/CT) and tumour vasculature (DCE-CT). Materials and methods Thirty-six non-small cell lung cancer (NSCLC) patients underwent functional imaging prior to radical radiotherapy. Kinetic analysis was performed on DCE-CT scans to acquire blood flow (BF) and volume (BV) maps. HX4 PET/CT and DCE-CT scans were nonrigidly coregistered to the planning FDG PET/CT. Two clustering steps were performed on multiparametric images: first to segment each tumour into homogeneous subregions (i.e. supervoxels) and second to group the supervoxels of all tumours into phenotypic clusters. Patients were split based on the absolute or relative volume of supervoxels in each cluster. Overall survival was compared using a log-rank test.

Results Unsupervised clustering of supervoxels yielded four independent clusters. One cluster (high hypoxia, high FDG, intermediate BF/BV) related to a high-risk tumour type: patients assigned to this cluster had significantly worse survival compared to patients not in this cluster $(\mathrm{p}=0.035)$.
\end{abstract}

Conclusions We designed a subregional analysis for multiparametric imaging in NSCLC, and showed the potential of subregion classification as a biomarker for prognosis. This methodology allows for a comprehensive data-driven analysis of multiparametric functional images. 


\section{Introduction}

Tumours often display significant intratumour heterogeneity at the time of diagnosis. Genetic differences, in combination with regional variation in the tumour microenvironment, result in phenotypically distinct tumour subregions [1,2]. Characteristics such as metabolic activity, proliferation, cell death, and vasculature vary throughout the tumour, influencing the sensitivity to (radio)therapy [3]. Identifying therapy resistant subvolumes could give greater insight into the underlying biological processes that adversely affect patient outcome, and could propel development of personalized therapies [4] by, for example, escalating radiation dose in treatment resistant areas $[5,6]$ or by combining targeted drugs and dose modifications [7].

Imaging provides a minimally invasive way to quantify both anatomical and functional spatial variations in the tumour and tumour microenvironment. For instance, different PET and SPECT tracers allow visualization of a range of biological processes (e.g. metabolic activity, proliferation, and hypoxia), and dynamic contrastenhanced CT or MRI scans can demarcate vasculature of the tumour. Most imaging based features used in clinical practice neglect spatial differences; they only consider simple characteristics, such as maximum or average tumour values. Voxelwise approaches, on the other hand, use all available data, but are sensitive to noise. Subvolume analysis could combine the advantages of both strategies by reducing the influence of noise without neglecting intratumour heterogeneities.

Current methods to calculate regional imaging features to identify subvolumes are based on binary classifiers (i.e. presence or absence of a feature), threshold values $[8,9]$, geographical location (e.g. tumour rim versus core $[9,10]$ ), or data-driven approaches $[11,12]$. The first three methods all require a priori knowledge about the appropriate imaging features or thresholds, which needs to be derived from previously acquired data or biological insights [2]. These strategies can validate the utility of previously determined thresholds, however, they are limited in their ability to derive information from the images. Data-driven approaches, in contrast, do not require any prior knowledge and directly depend on the input images. Wu et al. showed the feasibility of data-driven approaches in lung cancer to determine tumour subregions based on CT and FDG PET [11]. However, a methodology to comprehensively assess subregions based on anatomical imaging in combination with multiple functional imaging modalities, including hypoxia and tumour vasculature, is still lacking.

Our aim was to derive a methodology to combine multiple functional imaging techniques to identify high-risk tumour subregions in NSCLC patients treated with (chemo)radiotherapy. We developed a data-driven clustering approach to correlate the subregions to patient prognosis using FDG PET/CT (metabolic activity), HX4 PET/CT (hypoxia), and dynamic contract-enhanced CT (DCE-CT; tumour vasculature). 


\section{Materials and methods}

\section{Patient selection and treatment}

We analysed 36 stage IB-IV NSCLC patients included in two prospective clinical trials (NCT01024829 [13] and NCT01210378). Both trials were approved by the Medical Ethics Review Committee and all patients gave written informed consent. The first trial was designed to improve local control by uniformly boosting the tumour or by boosting the high uptake area on FDG PET/CT. The patients received 66 Gy in 24 fractions with an integrated boost, with or without concurrent or sequential chemotherapy. The boost dose was maximized until normal tissue constraints were met. Patients that could not receive dose escalation were treated with the standard 66 Gy or lower in 24 fractions. The second clinical trial was designed to increase overall survival by adding a vasodilating drug to standard (chemo)radiotherapy. All curative radiotherapy schedules were allowed. One patient received stereotactic radiotherapy (60 Gy in 8 fractions). The other patients received 53-70.2 Gy in 30-42 fractions. The majority of the patients of both trials received concurrent chemotherapy; one patient received neoadjuvant chemotherapy, one patient sequential chemotherapy, and two patients were treated by radiotherapy only.

\section{Image acquisition}

The pretreatment imaging protocol was identical for the two clinical trials. All patients included in our analysis received a planning FDG PET/CT, HX4 PET/CT and DCE-CT scan, within a week before the start of radiotherapy. For one patient only, the time between the imaging exceeded one week (11 days). For all scans, the patients were placed in treatment position: on a flat table with a headrest, arm and knee supports.

A 4D respiratory gated FDG PET/CT was acquired for treatment planning purposes. One hour after injecting $201 \mathrm{MBq} \pm 49 \mathrm{MBq}$ FDG tracer, the patients were scanned on a Siemens Biograph 40 PET/CT scanner (Siemens Healthcare, Erlangen, Germany). The PET scans were corrected for attenuation using the midventilation phase of the 4D CT scan, scatter, and random coincidences. The PET images were reconstructed (OSEM or PSF algorithm) to 4 by $4 \mathrm{~mm}$ voxels, with a slice spacing of $3 \mathrm{~mm}$. The CT image had the same slice spacing with 1 by $1 \mathrm{~mm}$ voxels. PET standardized uptake values (SUV) were calculated by correcting for body weight, injected dose, and decay. The PET and the midventilation CT image were fused and used for the delineation of the gross tumour volume (GTV) of the primary tumour by an experienced radiation oncologist.

Hypoxia HX4 PET/CT scans and DCE-CT scans were acquired as part of a translational research programme of both clinical trials. For the hypoxia PET, patients were scanned 4 hours after injecting $418 \mathrm{MBq} \pm 75 \mathrm{MBq}$ of the HX4 PET tracer on a 
Gemini TF64 PET/CT scanner (Philips, Best, the Netherlands) [14, 15]. PET images were corrected for attenuation, using a low-dose CT scan, and for scatter and random coincidences. A BLOB-OS-TF algorithm (3 iterations and 33 subsets) was used to reconstruct PET voxels of 4 by $4 \mathrm{~mm}$ with a slice spacing of $4 \mathrm{~mm}$. Tumour-tobackground ratios (TBR) were calculated by dividing tumour SUV by the mean SUV in the aortic arch.

DCE-CT scans were acquired on a second or third generation dual source CT scanner (SOMATOM Definition Flash or SOMATOM Force, Siemens Healthcare, Erlangen, Germany). An $80 \mathrm{kVp}$ CT scan was acquired of the primary tumour every 1.5 seconds for 33 consecutive frames, after injecting 60 or $65 \mathrm{ml}$ iodine-based contrast material (Iopromide 300, Bayer Healthcare, Berlin, Germany). The contrast material $(7 \mathrm{ml} / \mathrm{s})$ was followed by injection of a saline chaser of 30 or $40 \mathrm{ml}(7 \mathrm{ml} / \mathrm{s})$. The scans were reconstructed to $0.8 \times 0.8 \mathrm{~mm}$ voxels with a slice thickness of $5 \mathrm{~mm}$, using a B20f filter. Acquisition was performed in expiration breath hold. If breath hold could not be maintained, patients were asked to continue shallow breathing. All 33 scans were aligned to the first scan using a fluid-based registration to compensate for possible movement artefacts. Next, a noise reduction was performed (syngo MMWP, version VE40A; Siemens [16]). The registered images were smoothed with a median 5 voxel filter and resampled to a $256 \times 256$ grid $(1.6 \times 1.6 \mathrm{~mm})$. Kinetic analysis was performed on the preprocessed images, using an adiabatic approximation to the tissue homogeneity model (ATH) $[17,18]$. The arterial input function was determined by delineating a region of interest in the aortic arch. Finally, parametric maps were calculated for tumour blood flow (BF), in $\mathrm{ml} / 100 \mathrm{~g} / \mathrm{min}$, and tumour blood volume (BV), in $\mathrm{ml} / 100 \mathrm{~g}$.

\section{Image registration and supervoxel segmentation}

All image modalities were registered to the planning CT scan. The CT scan of the HX4 PET/CT and the first frame of the DCE-CT scan were first aligned to the planning CT scan, and thereafter, deformed using a non-rigid registration using the Elastix toolbox [19, 20]. The HX4 PET scan, and the BF and BV maps were deformed accordingly. The voxels were resampled to create isotropic voxels of the inplane dimensions of the planning CT scan $(1 \times 1 \times 1 \mathrm{~mm})$. A weighted average image was constructed by normalizing each image, by calculating z-scores, and summing these Z-score maps. The weighted average image was used to subdivide the GTV using so-called supervoxels; similar voxels were clustered into homogeneous 3D patches preserving local boundaries. This strategy was chosen to minimize the effect of registration uncertainties, reduce the amount of data, and reduce noise. An adapted kmeans clustering algorithm called Simple Linear Iterative Clustering (SLIC [21]), with 
a compactness of 20 and size of 400 voxels (typically $0.4 \mathrm{~cm}^{3}$ ), was used to create supervoxels. Only supervoxels with at least $90 \%$ of their voxels in the GTV of the primary tumour and CT Hounsfield units larger than $100 \mathrm{HU}$, to exclude partial volume effects of lung tissue, were used for further analysis. Furthermore, supervoxels with a high residual in the DCE-CT kinetic analysis $(>100 \times 33)$, i.e. squared-difference of the calculated and measured tumour enhancement, were excluded from analysis. Meaning that at each time point, a maximum difference between the fitted and observed enhancement of $10 \mathrm{HU}$ was allowed. For voxels exceeding this empirical threshold, no accurate fit was expected to be achieved either because of the model assumptions were violated or because the signal was too low in that voxel. The uncertainty of the model strongly depends on noise levels and increases as the signalto-noise ratio drops (SNR < 5) [22]. For each supervoxel, the median image values of the voxels within that supervoxel were calculated for the midventilation scan of the planning CT (HU), FDG PET (SUV), HX4 PET (TBR), BV (ml/100 g) and BF $(\mathrm{ml} / 100 \mathrm{~g} / \mathrm{min})$.

\section{Clustering of the supervoxels and linking to overall survival}

The median supervoxel image values were normalized by calculating z-scores. Next, the normalized image values of all patients were grouped using a hybrid hierarchical clustering. This unsupervised clustering approach first creates mutual clusters, i.e. points closely related to each other that cannot be separated, and then performs a topdown clustering based on those small basic elements [23]. To determine the number of clusters to use for further analysis we optimized the between and within cluster sum of squares (Calinski and Harabasz index [24]) and visually assessed the dendrogram. Clusters were assigned to all supervoxels of all patients. Thereafter, the tumours were assigned to one or more clusters depending on the absolute or relative volume of supervoxels present from each cluster. We assigned a tumour to a cluster if it contained a significant amount of supervoxels of that cluster: i.e. more than $50 \%$ of the tumour or more than the median volume over all patients.

Finally, a log-rank test was used for each cluster to assess overall survival differences between the patients assigned to the clusters versus patients not belonging to those clusters. The overall survival time was defined as the time between pathologic diagnosis and death from any cause. A graphical representation of the workflow is shown in Figure 7.1. The clustering and survival analysis was performed using R: A Language and Environment for Statistical Computing (v3.3.2, Vienna, Austria). 


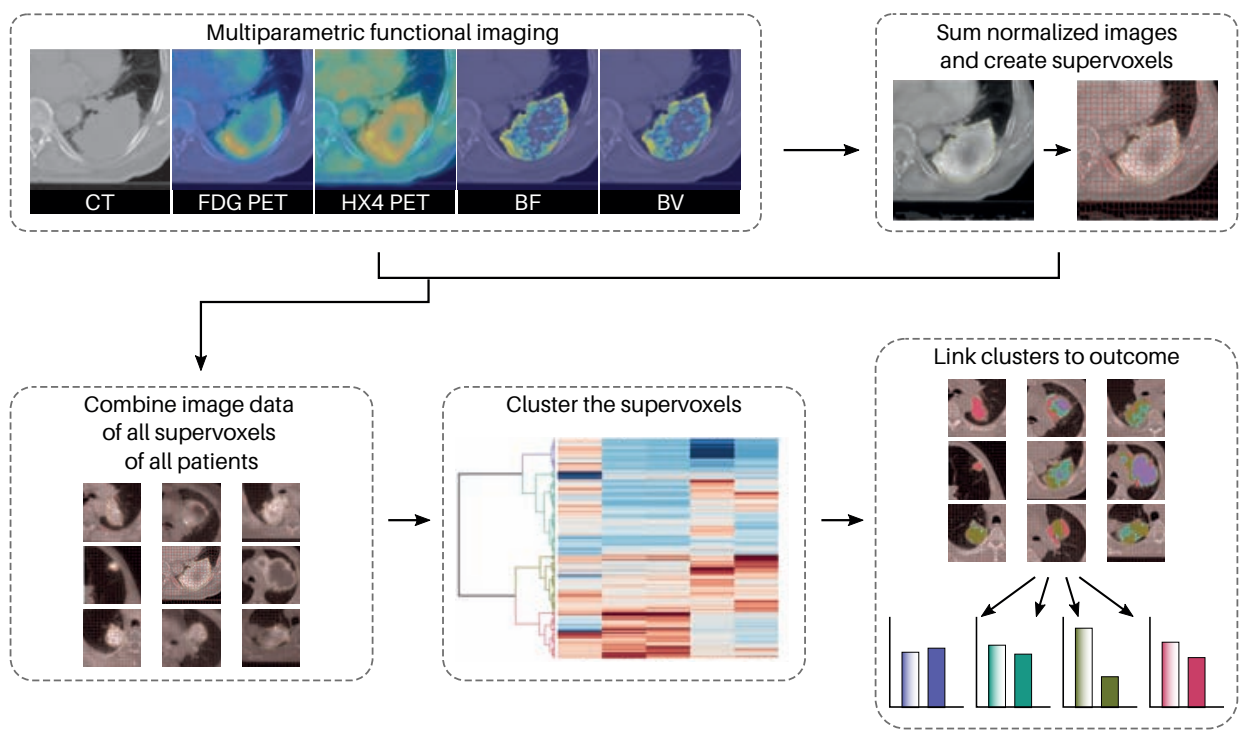

Figure 7.1. The processing workflow. For each patient, the multiparametric images are normalized and summed, and used to create supervoxels (top row). For each supervoxel, the median of all image features are calculated. Next, the supervoxels of all patients are combined and clustered based on their image features. Patients are assigned to the different clusters and differences in overall survival between the groups can be assessed.

\section{Results}

The GTV ranged from 3 to $1449 \mathrm{~cm}^{3}$ (median $53 \mathrm{~cm}^{3}$ ). For all patients, supervoxels could be created, ranging from 4 to 2028 (median 89) supervoxels per tumour. After removing the supervoxels with high DCE-CT residuals or low CT HU, one patient had zero supervoxels and was excluded from further analysis. This tumour was relatively small $\left(6.9 \mathrm{~cm}^{3}\right)$ and had initially only 4 supervoxels. The number of supervoxels for the remaining 35 patients ranged from 1 to 1744 (median 60). More patient and tumour details for these 35 patients and the analysed supervoxels are listed in Table 7.1.

The unsupervised clustering of all supervoxels over all patients provided four independent groups see Figure 7.2, determined by the local maximum of the Calinski and Harabasz index. The first cluster (purple) was characterized by low blood flow and blood volume, and low PET uptake values. The second cluster (turquoise) had low perfusion values and intermediate PET uptake values; the third cluster (olive green) had intermediate perfusion values and the highest HX4 SUV and FDG SUV; the fourth cluster (pink) had a relatively high blood flow and blood volume with intermediate PET uptake values. The CT values were relatively heterogeneous in each cluster. See also Table 7.2 for the average values and standard deviations per cluster. The first cluster 
Table 7.1. Patient and tumour characteristics of the 35 patients used for the cluster analysis.

\begin{tabular}{llr}
\hline Patients & & 35 \\
\hline Gender & Male & 26 \\
& Female & 9 \\
\hline GTV & Median volume $\left(\mathrm{cm}^{3}\right)$ & 53 \\
& Volume interquartile range $\left(\mathrm{cm}^{3}\right)$ & $33-113$ \\
& Median number of supervoxels & 60 \\
& Supervoxel interquartile range & $19-135$ \\
\hline \multirow{2}{*}{ TNM (T) } & T2 & 11 \\
& T3 & 12 \\
& T4 & 12 \\
\hline \multirow{2}{*}{ TNM (N) } & N0 & 3 \\
& N1 & 2 \\
& N2 & 20 \\
& N3 & 10 \\
\hline TNM (M) & M0 & 28 \\
& M1 & 7 \\
\hline Pathology & Adenocarcinoma & 13 \\
& Squamous cell carcinoma & 11 \\
& Large-cell carcinoma & 6 \\
& NSCLC not otherwise specified & 5 \\
\hline
\end{tabular}

Table 7.2. Image feature averages and standard deviations over the supervoxels per cluster.

\begin{tabular}{lccccc}
\hline Cluster & $\begin{array}{c}\mathrm{CT} \\
(\mathrm{HU})\end{array}$ & $\begin{array}{c}\mathrm{BF} \\
(\mathrm{ml} / 100 \mathrm{~g} / \mathrm{min})\end{array}$ & $\begin{array}{c}\mathrm{BV} \\
(\mathrm{ml} / \mathrm{l00} \mathrm{g})\end{array}$ & $\begin{array}{c}\mathrm{HX} 4 \\
(\mathrm{TBR})\end{array}$ & $\begin{array}{c}\text { FDG } \\
(\mathrm{SUV})\end{array}$ \\
\hline 1 & $32.3 \pm 8.5$ & $15.6 \pm 7.4$ & $1.2 \pm 0.6$ & $0.4 \pm 0.2$ & $1.4 \pm 1.1$ \\
2 & $18.3 \pm 22.0$ & $24.3 \pm 12.5$ & $2.6 \pm 1.5$ & $1.1 \pm 0.2$ & $4.4 \pm 2.2$ \\
3 & $28.9 \pm 12.7$ & $45.4 \pm 15.1$ & $5.3 \pm 1.8$ & $1.4 \pm 0.3$ & $8.4 \pm 3.7$ \\
4 & $29.0 \pm 19.3$ & $78.7 \pm 30.4$ & $8.9 \pm 3.3$ & $1.0 \pm 0.2$ & $4.4 \pm 2.0$ \\
\hline
\end{tabular}

(purple) is mainly dominated by one patient (717/808 supervoxels: $89 \%$ ), which is shown in green in Figure 7.2. This patient had the largest number of supervoxels (1744; $854 \mathrm{~cm}^{3}$ ) (third patient in Figure 7.3). In the other clusters, there was a more even contribution of a mix of patients.

With all supervoxels assigned to a cluster, we could evaluate the contribution of each cluster to each tumour. In Figure 7.3, three example patients are shown with the supervoxel segmentation and assignment of the supervoxels to one of the four clusters. Four patients had supervoxels only belonging to one supervoxel cluster. For the other 31 patients, a mix of two, three or four clusters was identified; see Figure 7.3 and Supplementary Table S7.1. 

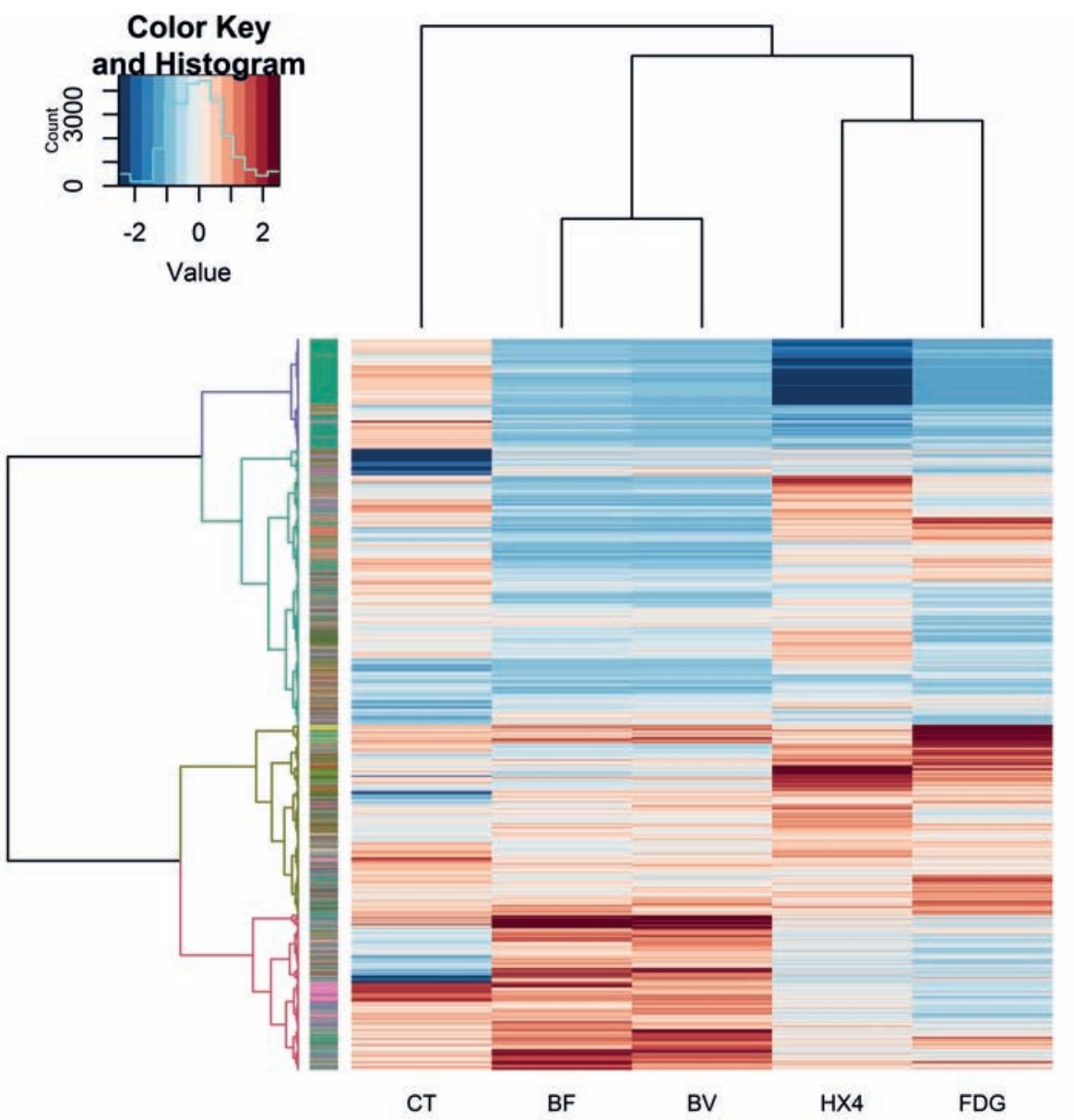

Figure 7.2. Heatmap and dendrogram of all supervoxels of all patients. Each row is one supervoxel. The imaging values are normalized z-scores of the median of the voxels in one supervoxel. The histogram and colour range of all normalized values is show in the top left. The dendrogram is divided in four clusters, indicated by the four colours of the dendrogram (cluster 1: purple; cluster 2: turquoise; cluster 3: olive green; cluster 4: pink). The first coloured bar indicates the patient the supervoxel belongs to: every colour is a different patient. 

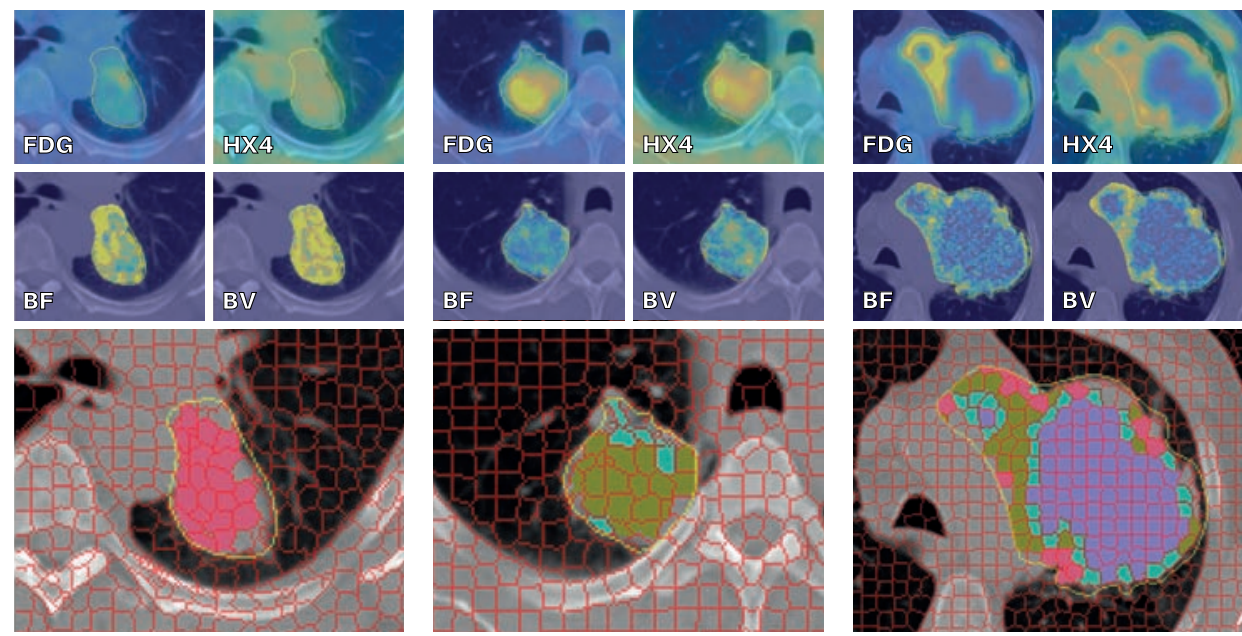

Figure 7.3. Three example patients of the clustered supervoxels. The two top rows show the functional images (FDG PET, HX4 PET, blood flow, and blood volume) overlaid on the CT image. The same scaling is applied for each imaging modality for all three patients. The multiparametric images are used as input to create the four supervoxel clusters as shown in the bottom row. One tumour can consist of only one type of supervoxels (left), or a mix of two, three or four clusters. In this example, the first patient belongs to the pink cluster, the second patient to the turquoise and olive green cluster, and the third patient to all four clusters.

Based on the supervoxel distributions, patients were assigned to the different clusters. For one of the clusters (cluster 3; olive green), a significant survival difference was observed. Patients $(n=17)$ with a high relative or absolute volume of cluster 3 had a significant worse survival compared to patients not assigned to this cluster $(n=18)$ ( $\mathrm{p}=0.035$ ). This cluster was mainly characterized by a high HX4 uptake (average TBR of 1.4), and a high FDG SUV (average SUV of 8.4). At two years, the patients not in this cluster had a 2.6 times higher survival probability (67\%), compared to patients with a significant amount of supervoxels of this high-risk subgroup (26\%). For the other three clusters, no survival difference was found with a log-rank p-value of $0.935,0.730$ and 0.465 for respectively, cluster 1 (purple), cluster 2 (turquoise) and cluster 4 (pink).

\section{Discussion}

We explored a data-driven approach to identify tumour subregions with characteristic phenotypes based on pretreatment multiparametric imaging, and related these subregions to overall survival of NSCLC patients treated with definitive (chemo)radiation. Our method was designed to reduce the influence of registration uncertainties and of noise, while maintaining subregional information, by clustering the voxels into supervoxels. The methodology allowed for combining data of the whole population by 
clustering the supervoxels of all patients, in order to generate population based phenotypical subtypes. We found four distinct phenotypical clusters: one indicative for necrosis (low blood flow/volume and low PET uptake), one with low perfusion and intermediate PET values, one solely indicative of the high PET uptake, and one associated with relatively high tumour perfusion.

We could link these supervoxels subtype clusters to patient prognosis. Patients with subregions with a high HX4 TBR, FDG SUV and intermediate BF and BV, had a significantly worse prognosis compared to patients without a high absolute or relative volume of these subregions. Our findings are in agreement with Wu et al. who found in a similar type of analysis for FDG PET/CT that the most metabolically active and metabolically heterogeneous subvolumes were prognostic for overall survival [11]. In addition, we found that these metabolically active regions are highly hypoxic with a rather average blood perfusion and blood volume. Hypoxia is a well-known factor resulting in a worse prognosis in most solid tumours [25]. In NSCLC, a variety of hypoxia PET tracers was used to evaluate overall survival or progression free survival. A significantly worse overall survival was found for patients with tumours with high TBR values $[26,27]$. Also for progression free survival, a significant survival disadvantage was observed for patients with higher hypoxia SUV or TBR values [26, 28, 29]. All above studies showed a survival difference between hypoxic and non-hypoxic tumours, however, the thresholds varied between studies and hypoxia tracers. For FDG PET, only a subset of studies evaluating $\operatorname{SUV}_{\max }$ found this to be prognostic [26,27]. The tumour volume with an increased metabolic activity was shown as independent prognostic factor for survival in several studies. In these studies, patients with a larger metabolic volume generally had a worse prognosis [30, 31]. Only few studies have been published assessing the prognostic value of pretreatment DCE-CT imaging. Win et al. found that low tumour permeability was prognostic for survival [32]. Lazanyi et al. found a negative correlation between blood volume and progression in a subset of patients, and a negative correlation between $\mathrm{K}^{\text {trans }}$ and progression and overall survival for all patients [33]. We did not study tumour permeability, due to limited DCE-CT acquisition time optimized for blood flow and volume characterization, and could not reproduce this effect in the cluster related to survival. Although one cluster (cluster 4) showed increased perfusion values, no clear effect in outcome was observed.

One major advantage of our method is that defining thresholds is not required; no prior knowledge of image features is necessary. The unsupervised clustering method groups supervoxels based on the underlying data and the created clusters can be linked to outcome. However, the analysis does require some user input at different levels of the workflow. First, to create the supervoxels, we evaluated different combinations of size and compactness and visually assessed the results by inspecting adherence to local boundaries. We aimed to make the clusters small enough to maintain local 
structures, while large enough to reduce noise. Next, the hybrid hierarchical clustering creates a tree, a dendrogram, of clusters, which can be cut at every height depending on the desired number of clusters. Large clusters are more heterogeneous, while small clusters are more difficult to link to outcome. We optimized this by evaluating the within and between cluster sum of squares. Finally, the patients were assigned to the different clusters based on their supervoxel distribution. We decided to group the patients that had either a high absolute or high relative volume of supervoxels of a certain cluster. We tested the prognostic value of the total tumour volume to confirm that the observed results are not driven by tumour volume and found no significant value $(\mathrm{p}=0.327)$. The assignment of the patients to the different clusters could be further evaluated and optimized with more patients available.

One factor that needs to be stressed is that in this study we analysed patients of two clinical trials receiving either dose escalation based on FDG PET/CT, with or without concurrent or sequential chemotherapy, or standard (chemo)radiation with addition of a vasodilating drug. The heterogeneity in treatments and dose ranges could have influenced the patient prognosis. The cohort is however too small to study these influences. In addition, for the clustering, all supervoxels of all patients were combined, increasing the influence of the larger tumours on the clustering results.

The data set used is rather unique with 36 NSCLC patients scanned shortly before treatment using four different imaging modalities. Such a dataset is difficult to obtain and only possible in a dedicated research setting. Our clustering methodology, however, could be applied on any multiparametric imaging set, pre- or posttreatment imaging. The availability of multiparametric imaging will probably increase in the future, especially with for example MRI techniques that allow multiple sequences within the same session to acquire a range of functional images. With the increasing availability of multiparametric imaging, there will be a need for comprehensive analysis methods of these images.

To conclude, we designed a data-driven methodology for the analysis of pretreatment multiparametric imaging data in NSCLC patients on a subregional level. We showed that such an intratumour classification of heterogeneous subregions may allow to predict NSCLC patient prognosis after (chemo)radiation. This technique permits further insight into the underlying biological characteristics using an advanced analysis technique for multiparametric functional images.

\section{Acknowledgements}

Authors acknowledge financial support from ERC advanced grant (ERC-ADG-2015, $\mathrm{n}^{\circ} 694812$ - Hypoximmuno) and the QuIC-ConCePT project, which is partly funded by EFPI A companies and the Innovative Medicine Initiative Joint Undertaking (IMI 
$\mathrm{JU}-\mathrm{n}^{\circ}$ 115151). This research is also supported by the Dutch Technology Foundation STW (grant $\mathrm{n}^{\circ}$ P14-19 Radiomics STRaTegy), which is the applied science division of NWO, and the Technology Programme of the Ministry of Economic Affairs. Authors also acknowledge financial support from the EU 7th framework program (ARTFORCE - $\mathrm{n}^{\circ}$ 257144), SME Phase 2 (RAIL - $\mathrm{n}^{\circ}$ 673780), EUROSTARS (DART), the European Program H2020-2015-17 (ImmunoSABR - n 733008 and PREDICT - ITN - n 766276), Interreg V-A Euregio Meuse-Rhine ("Euradiomics"). We thank Robert Jeraj and the IGT group at the University of Madison, Wisconsin for providing the DCE-CT modelling. 


\section{References}

1. Vogelstein, B., Papadopoulos, N., Velculescu, V. E., Zhou, S., Diaz L. A., J. \& Kinzler, K. W. Cancer genome landscapes. Science 339, 1546-58 (2013).

2. O’Connor, J. P. B., Rose, C. J., Waterton, J. C., Carano, R. A. D., Parker, G. J. M. \& Jackson, A. Imaging intratumor heterogeneity: role in therapy response, resistance, and clinical outcome. Clin Cancer Res 21, 249-57 (2015).

3. Moding, E. J., Kastan, M. B. \& Kirsch, D. G. Strategies for optimizing the response of cancer and normal tissues to radiation. Nat Rev Drug Discov 12, 526-42 (2013).

4. Lambin, P., van Stiphout, R. G. P. M., Starmans, M. H. W., et al. Predicting outcomes in radiation oncology-multifactorial decision support systems. Nat Rev Clin Oncol 10, 27-40 (2013).

5. Even, A. J. G., van der Stoep, J., Zegers, C. M. L., et al. PET-based dose painting in non-small cell lung cancer: Comparing uniform dose escalation with boosting hypoxic and metabolically active sub-volumes. Radiother Oncol 116, 281-6 (2015).

6. Møller, D. S., Nielsen, T. B., Brink, C., et al. Heterogeneous FDG-guided doseescalation for locally advanced NSCLC (the NARLAL2 trial): design and early dosimetric results of a randomized, multi-centre phase-III study. Radiother Oncol 124, 311-7 (2017).

7. Yaromina, A., Granzier, M., Biemans, R., et al. A novel concept for tumour targeting with radiation: inverse dose-painting or targeting the "Low Drug Uptake Volume”. Radiother Oncol (2017).

8. Van Elmpt, W., Zegers, C. M. L., Reymen, B., et al. Multiparametric imaging of patient and tumour heterogeneity in non-small-cell lung cancer: quantification of tumour hypoxia, metabolism and perfusion. Eur J Nucl Med Mol Imaging 43, 240-8 (2016).

9. Metz, S., Ganter, C., Lorenzen, S., et al. Multiparametric MR and PET imaging of intratumoral biological heterogeneity in patients with metastatic lung cancer using voxel-by-voxel analysis. PLoS One 10, e0132386 (2015).

10. Gaustad, J. V., Benjaminsen, I. C., Graff, B. A., Brurberg, K. G., Ruud, E. B. M. \& Rofstad, E. K. Intratumor heterogeneity in blood perfusion in orthotopic human melanoma xenografts assessed by dynamic contrast-enhanced magnetic resonance imaging. J Magn Reson Imaging 21, 792-800 (2005).

11. Wu, J., Gensheimer, M. F., Dong, X., et al. Robust intratumor partitioning to identify high-risk subregions in lung cancer: a pilot study. Int J Radiat Oncol Biol Phys 95, 1504-12 (2016). 
12. Torheim, T., Groendahl, A. R., Andersen, E. K. F., et al. Cluster analysis of dynamic contrast enhanced MRI reveals tumor subregions related to locoregional relapse for cervical cancer patients. Acta Oncol 55, 1294-8 (2016).

13. Van Elmpt, W., De Ruysscher, D., van der Salm, A., et al. The PET-boost randomised phase II dose-escalation trial in non-small cell lung cancer. Radiother Oncol 104, 67-71 (2012).

14. Zegers, C. M. L., van Elmpt, W., Wierts, R., et al. Hypoxia imaging with $\left[{ }^{18} \mathrm{~F}\right] \mathrm{HX} 4$ PET in NSCLC patients: defining optimal imaging parameters. Radiother Oncol 109, 58-64 (2013).

15. Zegers, C. M. L., van Elmpt, W., Szardenings, K., et al. Repeatability of hypoxia PET imaging using $\left[{ }^{18} \mathrm{~F}\right] \mathrm{HX} 4$ in lung and head and neck cancer patients: a prospective multicenter trial. Eur J Nucl Med Mol Imaging 42, 1840-9 (2015).

16. Saddi, K. A., Chefd'hotel, C. \& Cheriet, F. Large deformation registration of contrast-enhanced images with volume-preserving constraint in Medical Imaging 2007: Image Processing 6512 (SPIE, 2007), 651203.

17. St Lawrence, K. S. \& Lee, T. Y. An adiabatic approximation to the tissue homogeneity model for water exchange in the brain: I. Theoretical derivation. J Cereb Blood Flow Metab 18, 1365-77 (1998).

18. La Fontaine, M. D., McDaniel, L. S., Kubicek, L. N., Chappell, R. J., Forrest, L. J. \& Jeraj, R. Patient characteristics influencing the variability of distributed parameter-based models in DCE-CT kinetic analysis. Vet Comp Oncol 15, 105-17 (2017).

19. Klein, S., Staring, M., Murphy, K., Viergever, M. A. \& Pluim, J. P. W. Elastix: a toolbox for intensity-based medical image registration. IEEE Trans Med Imaging 29, 196205 (2010).

20. Staring, M., Bakker, M. E., Stolk, J., Shamonin, D. P., Reiber, J. H. C. \& Stoel, B. C. Towards local progression estimation of pulmonary emphysema using CT. Med Phys 41, 021905 (2014).

21. Achanta, R., Shaji, A., Smith, K., Lucchi, A., Fua, P. \& Susstrunk, S. SLIC superpixels compared to state-of-the-art superpixel methods. IEEE Trans Pattern Anal Mach Intell 34, 2274-82 (2012).

22. La Fontaine, M. D. The viability of DCE-CT kinetic analysis in tumor vasculature imaging in veterinary medicine $\mathrm{PhD}$ thesis (University of Wisconsin-Madison, 2014).

23. Chipman, H. \& Tibshirani, R. Hybrid hierarchical clustering with applications to microarray data. Biostatistics 7, 286-301 (2006). 
24. Caliński, T. \& Harabasz, J. A dendrite method for cluster analysis. Communications in Statistics 3, 1-27 (1974).

25. Horsman, M. R., Mortensen, L. S., Petersen, J. B., Busk, M. \& Overgaard, J. Imaging hypoxia to improve radiotherapy outcome. Nat Rev Clin Oncol 9, 674-87 (2012).

26. Kinoshita, T., Fujii, H., Hayashi, Y., Kamiyama, I., Ohtsuka, T. \& Asamura, H. Prognostic significance of hypoxic PET using ${ }^{18} \mathrm{~F}$-FAZA and ${ }^{64} \mathrm{Cu}$-ATSM in nonsmall-cell lung cancer. Lung Cancer 91, 56-66 (2016).

27. Li, L., Hu, M., Zhu, H., Zhao, W., Yang, G. \& Yu, J. Comparison of $\left[{ }^{18} \mathrm{~F}\right]-$ fluoroerythronitroimidazole and $\left[{ }^{18} \mathrm{~F}\right]$-fluorodeoxyglucose positron emission tomography and prognostic value in locally advanced non-small-cell lung cancer. Clin Lung Cancer 11, 335-40 (2010).

28. Vera, P., Thureau, S., Chaumet-Riffaud, P., et al. Phase II study of a radiotherapy total dose increase in hypoxic lesions identified by F-miso PET/CT in patients with non-small cell lung carcinoma [RTEP5 study]. J Nucl Med 58, 1045-53 (2017).

29. Eschmann, S. M., Paulsen, F., Reimold, M., et al. Prognostic impact of hypoxia imaging with ${ }^{18} \mathrm{~F}$-misonidazole PET in non-small cell lung cancer and head and neck cancer before radiotherapy. J Nucl Med 46, 253-60 (2005).

30. Bazan, J. G., Duan, F., Snyder, B. S., et al. Metabolic tumor volume predicts overall survival and local control in patients with stage III non-small cell lung cancer treated in ACRIN 6668/RTOG 0235. Eur J Nucl Med Mol Imaging 44, 17-24 (2017).

31. Ohri, N., Duan, F., Machtay, M., et al. Pretreatment FDG-PET metrics in stage III non-small cell lung cancer: ACRIN 6668/RTOG 0235. J Natl Cancer Inst 107, djv004 (2015).

32. Win, T., Miles, K. A., Janes, S. M., et al. Tumor heterogeneity and permeability as measured on the CT component of PET/CT predict survival in patients with non-small cell lung cancer. Clin Cancer Res 19, 3591-9 (2013).

33. Lazanyi, K. S., Abramyuk, A., Wolf, G., et al. Usefulness of dynamic contrast enhanced computed tomography in patients with non-small-cell lung cancer scheduled for radiation therapy. Lung Cancer 70, 280-5 (2010). 


\section{Supplementary data}

Table S7.1. Number of supervoxels per cluster (C1-C4) and assignment of the patients to the four clusters. Patients can be assigned to multiple clusters.

\begin{tabular}{|c|c|c|c|c|c|c|c|c|}
\hline \multirow[t]{2}{*}{ Patient } & \multicolumn{4}{|c|}{ Number of supervoxels } & \multicolumn{4}{|c|}{$\begin{array}{c}\text { Patients assigned to cluster } \\
(>\text { median supervoxels or }>50 \%)\end{array}$} \\
\hline & $\mathrm{C} 1$ & C2 & C3 & $\mathrm{C} 4$ & $\mathrm{Cl}$ & C2 & C3 & $\mathrm{C} 4$ \\
\hline 1 & 0 & 16 & 16 & 28 & 0 & 0 & 0 & 1 \\
\hline 2 & 0 & 4 & 0 & 0 & 0 & 1 & 0 & 0 \\
\hline 3 & 0 & 122 & 70 & 10 & 0 & 1 & 1 & 0 \\
\hline 4 & 1 & 82 & 83 & 1 & 1 & 1 & 1 & 0 \\
\hline 5 & 0 & 8 & 0 & 11 & 0 & 0 & 0 & 1 \\
\hline 6 & 0 & 1 & 0 & 0 & 0 & 1 & 0 & 0 \\
\hline 7 & 0 & 97 & 34 & 175 & 0 & 1 & 1 & 1 \\
\hline 8 & 0 & 0 & 1 & 1 & 0 & 0 & 0 & 0 \\
\hline 9 & 0 & 0 & 1 & 5 & 0 & 0 & 0 & 1 \\
\hline 10 & 0 & 0 & 2 & 9 & 0 & 0 & 0 & 1 \\
\hline 11 & 14 & 69 & 16 & 188 & 1 & 1 & 0 & 1 \\
\hline 12 & 0 & 0 & 1 & 0 & 0 & 0 & 1 & 0 \\
\hline 13 & 1 & 22 & 0 & 3 & 1 & 1 & 0 & 0 \\
\hline 14 & 0 & 10 & 1 & 7 & 0 & 1 & 0 & 0 \\
\hline 15 & 5 & 16 & 0 & 13 & 1 & 0 & 0 & 1 \\
\hline 16 & 16 & 42 & 22 & 20 & 1 & 1 & 1 & 1 \\
\hline 17 & 0 & 5 & 83 & 29 & 0 & 0 & 1 & 1 \\
\hline 18 & 0 & 25 & 14 & 0 & 0 & 1 & 0 & 0 \\
\hline 19 & 12 & 407 & 245 & 63 & 1 & 1 & 1 & 1 \\
\hline 20 & 0 & 11 & 21 & 6 & 0 & 0 & 1 & 0 \\
\hline 21 & 0 & 27 & 51 & 4 & 0 & 1 & 1 & 0 \\
\hline 22 & 717 & 468 & 339 & 220 & 1 & 1 & 1 & 1 \\
\hline 23 & 2 & 46 & 70 & 24 & 1 & 1 & 1 & 1 \\
\hline 24 & 0 & 0 & 1 & 85 & 0 & 0 & 0 & 1 \\
\hline 25 & 19 & 275 & 101 & 3 & 1 & 1 & 1 & 0 \\
\hline 26 & 0 & 5 & 38 & 36 & 0 & 0 & 1 & 1 \\
\hline 27 & 1 & 0 & 1 & 0 & 1 & 0 & 0 & 0 \\
\hline 28 & 0 & 62 & 5 & 5 & 0 & 1 & 0 & 0 \\
\hline 29 & 0 & 20 & 3 & 21 & 0 & 1 & 0 & 1 \\
\hline 30 & 0 & 3 & 17 & 2 & 0 & 0 & 1 & 0 \\
\hline 31 & 0 & 7 & 83 & 25 & 0 & 0 & 1 & 1 \\
\hline 32 & 0 & 12 & 1 & 16 & 0 & 0 & 0 & 1 \\
\hline 33 & 19 & 122 & 20 & 101 & 1 & 1 & 1 & 1 \\
\hline 34 & 1 & 4 & 0 & 0 & 1 & 1 & 0 & 0 \\
\hline 35 & 0 & 40 & 60 & 28 & 0 & 1 & 1 & 1 \\
\hline Median & 0 & 16 & 16 & 10 & & & & \\
\hline Patients per cluster & & & & & 12 & 20 & 17 & 19 \\
\hline
\end{tabular}




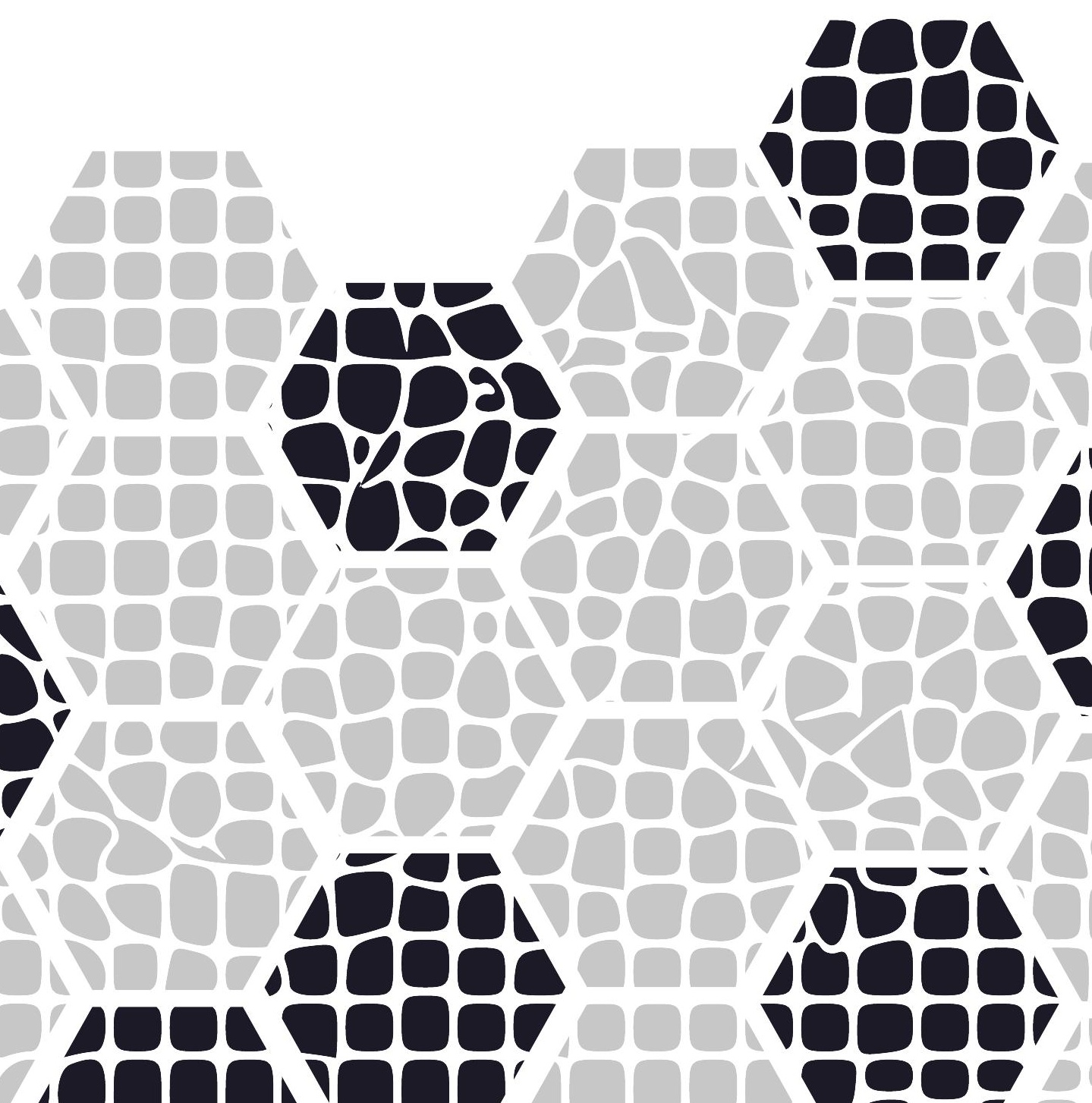




\section{Discussion}
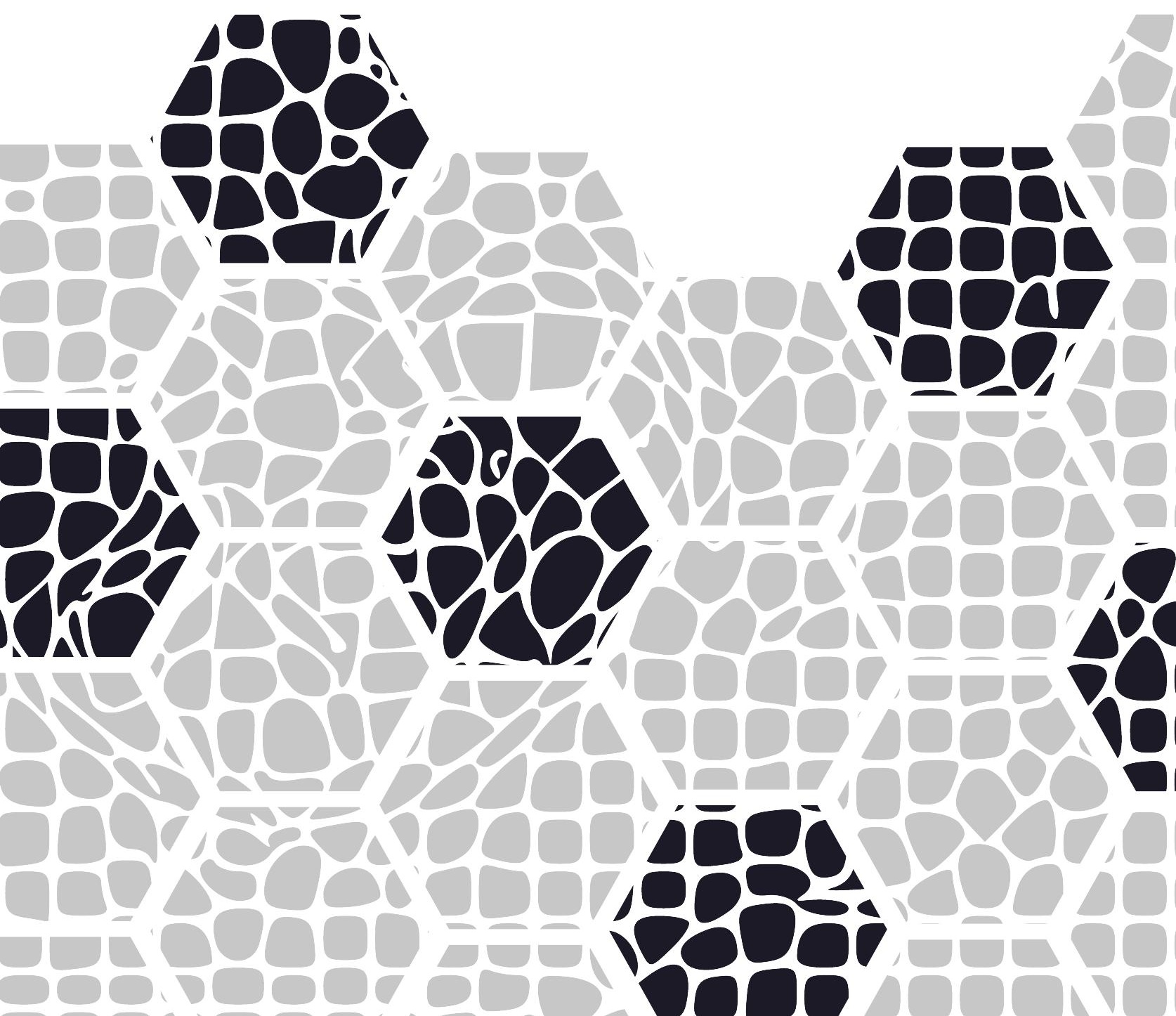


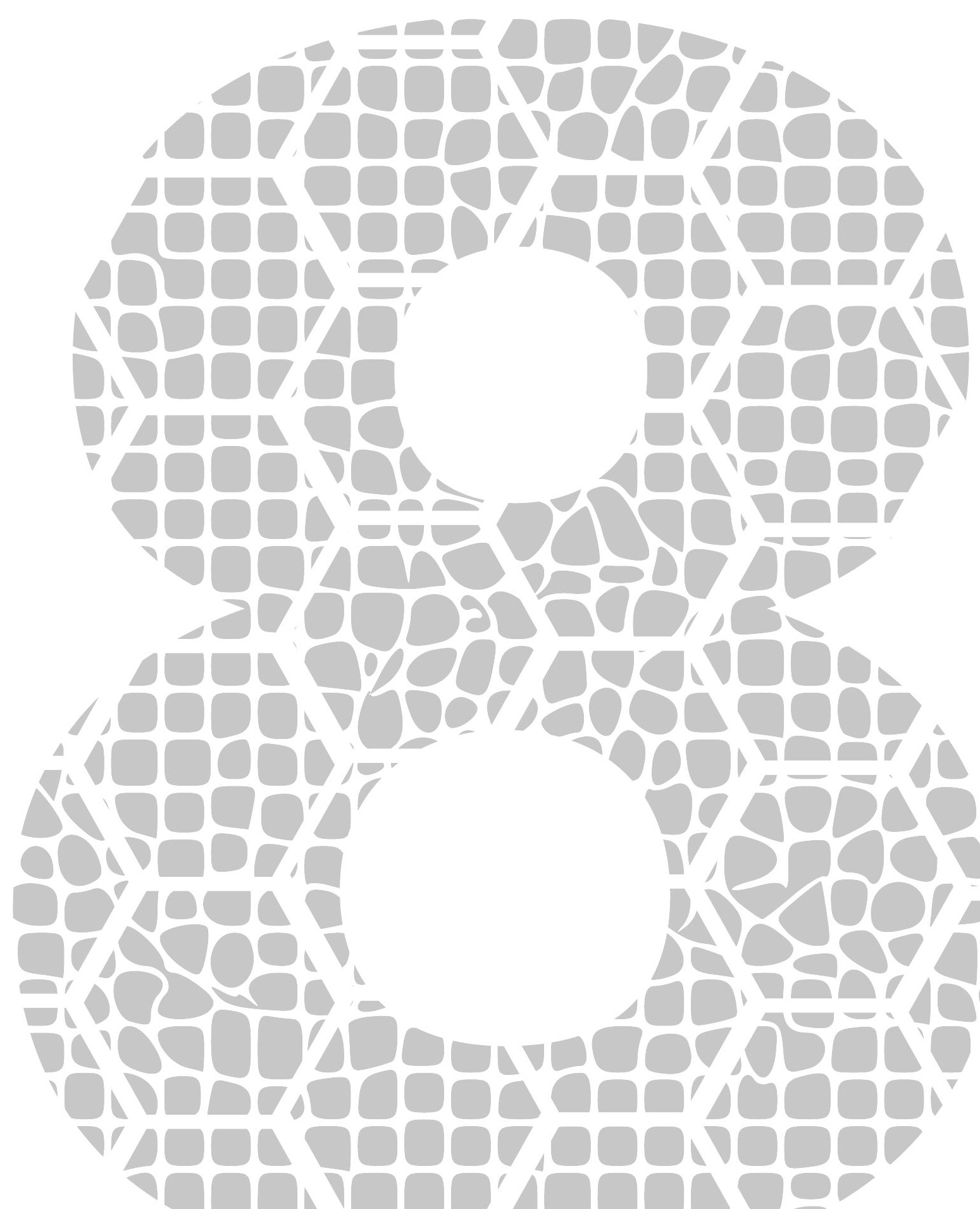




\section{The promise of multiparametric imaging in oncology: how do we move forward?}

Aniek J.G. Even, Dirk De Ruysscher, Wouter van Elmpt

Adlapted from

Editorial commentary in European Journal of Nuclear Medicine and Molecular Imaging, 2016 July; 43(7): 1195-1198 DOI: $10.1007 / \mathrm{s} 00259-016-3361-1$ 


\section{Background}

With the introduction of combined imaging modalities including PET/CT and more recently PET/MRI, there is strong interest in utilizing these independent modalities in a synergistic way. Multiparametric imaging research has been mainly accelerated by the MRI community, as MRI offers the possibility to visualize diverse properties of tissues by acquiring different sequences (e.g. ADC mapping, T1/T2weighted images). More recently, several research papers have been published investigating multiparametric imaging in a variety of cancer types using combined nuclear medicine imaging techniques, with one or more radioactive tracers, together with advanced MR or CT imaging equipment [1-5]. The increased number of standardization (or harmonization) procedures that are being rolled out in all modalities are helping drive multiparametric imaging forward [6, 7]. These procedures should remove some of the uncertainties and difficulties that multicentre trials face in quantitative analysis and facilitate more robust quantitative imaging-derived parameters [8].

A multitude of imaging techniques are currently available for visualizing tumour morphology and biology in vivo. CT and MRI both provide morphological information. Biological processes that are dysregulated in cancer cells and distinguish malignant tissue from healthy tissue can be visualized using nuclear medicine-based techniques, MRI, and CT. For many years, PET tracers focussed on general features of tumours or normal tissues. The classic marker for ATP-independent GLUT-mediated deoxyglucose uptake, which is often upregulated in tumours, is FDG. Uptake of FDG is commonly considered an indicator of "metabolism" although it reflects only a small part of the metabolic process. Another tumour hallmark that can be visualized using PET imaging is the activity of thymidine kinase 1, an enzyme involved in DNA synthesis, as a surrogate for most cell proliferation. Fluorothymidine (FLT) is the most studied tracer for this purpose. Most solid tumours are, in addition, characterized by a low oxygen levels, i.e. hypoxia. Frequently used hypoxia PET tracers, based on 2-nitroimidazoles (e.g. FMISO, FAZA, HX4), visualize increased reductase activity in cells with insufficient oxygen, but do not show the molecular consequences of hypoxia. MRI and CT can be used for imaging of the tumour vasculature. The chaotic and poor vasculature of tumours is often inadequate and results in diffusionlimited or perfusion-limited hypoxia. Dynamic contrast-enhanced CT or MRI (DCE CT/MRI) allows investigation of the vasculature (e.g. tumour permeability and blood flow) by typically following injection of a contrast agent over time as it passes through the tumour blood vessels. Diffusion-weighted MRI (DWI MRI) facilitates mapping of diffusion patterns of water molecules, a characteristic related to cellular density. These advanced MRI techniques have a role in the standard guidelines for 
distinguishing malignant involvement from benign conditions in prostate cancer and pleural mesothelioma, for example $[9,10]$. Novel PET tracers have been developed for specific receptor imaging, including PET-labelled drugs. Examples include

${ }^{18} \mathrm{~F}$-erlotinib, ${ }^{89} \mathrm{Zr}$-bevacizumab, ${ }^{89} \mathrm{Zr}$-trastuzumab and ${ }^{68} \mathrm{Ga}$-PSMA. The last of these is increasingly used in oncology as it may lead to treatment changes [11].

Even though a wide range of imaging techniques are available, not many functional imaging markers have gained wide acceptance in standard practice, except FDG PET. Of all imaging techniques, no single modality or tracer can answer all questions, and so multimodality imaging should be individually tailored to the specific research question or clinical question.

\section{How to move forward}

It has been recognized for a long time that tumours are frequently heterogeneous in their presentation and behaviour. Multiparametric imaging allows investigation of this heterogeneity. Subvolumes with distinct properties inside tumours can be identified. For example, information on perfusion in subvolumes combined with hypoxia imaging may reveal differences between perfusion-limited hypoxia and intrinsic tumour hypoxia [12-14], or may explain the lack of accessibility of targeted agents to a receptor on the tumour cell.

The large amount of information we can currently gather from these advanced imaging techniques is undoubtedly interesting. However, the next question is how to identify which biological processes are relevant, how these processes influence patient prognosis, and finally how to individualize treatment based on this information. We envisage two distinct strategies to move forward.

\section{Strategy 1: The agnostic data-driven approach}

A way to link imaging studies to a clinically relevant outcome is through so-called pattern of relapse studies. Such studies allow correlation of imaging features with the patient or tumour outcome (e.g. recurrence location) using follow-up imaging of various lesions [15-18]. Alternatively, the imaging characteristics with biological properties can be validated in a surgical specimen [19-22]. Either of these datadriven approaches are attractive because no complete molecular knowledge is needed: the observations drive the identification of treatment resistant areas in a pragmatic, agnostic way.

The drawback is clearly that no further biological precision can be obtained easily. Another downside of this approach is its data-driven nature: many patients need to be imaged before these patterns of relapse can be accurately quantified, especially when 
these features divide the cohort into smaller subgroups, resulting in insufficient data. A comprehensive understanding of the combined imaging modalities is also essential. All imaging modalities have their own inherent uncertainties, e.g. artefacts on MRI, limited resolution of PET, uncertainties in kinetic modelling with DCE CT/MRI. These uncertainties of the individual imaging modalities together with registration effects could explain why correlations found between imaging modalities at the tumour and subtumour levels are no longer present at the voxel level. For example, a simulation study showed blurring of the underlying pathology because of the limited resolution of PET scanners [23].

One possible way to integrate multiparametric imaging in treatment individualization is by using radiotherapy "dose painting". The radiation dose can be redistributed over different tumour regions according to the expected treatment sensitivity derived from imaging data to improve local tumour control without increasing toxicity. Several clinical trials are looking into this approach, and treatment personalization can be performed at different levels; ranging from the subvolume level to the very fine voxel level [24-26]. Similarly to the pattern-of-relapse studies, dose painting requires detailed analyses to find dose-response relationships between functional imaging, underlying pathology, and outcome to design appropriate clinical trials.

\section{Strategy 2: Biology-driven approach}

Up to the present date, clinical guidelines are written for a defined population, typically derived from phase III clinical trials. As subgroups become smaller eventually coming down to a personalized level, the agnostic data-driven approach is no longer possible. As all individuals and their disease are unique, so too will all tumours and even all tumour cells be found to be unique [27]. Tailoring a treatment for each cell and particularly proving that the outcome in the patient was due to this intervention would be impossible. However, there is an opportunity for molecular imaging in this area. It is possible to individualize the treatment by knowing more precisely the main molecular characteristics of a tumour that are distributed in a more or less homogeneous way throughout the tumour. A classical example is the exon 19 or 21 mutation of the EGFR gene in adenocarcinoma of the lung. While resistance will eventually occur in all patients, in the beginning, the mutations are found in nearly all tumour cells and are spread homogeneously throughout the tumour [28]. Before the tumour increases in size, it shows increasing inhomogeneity that can be visualized [29]. Before classical "disease progression" occurs, more precise imaging could identify patients who may be switched to an alternative therapy. It is expected that the combination of molecular knowledge underpinning the tumour's biology, specific imaging probes, and image analyses techniques will be an integral part of precision medicine. Multiparametric 
imaging might facilitate this approach by selecting the appropriate molecular imaging tools that quantify tumour properties or stratify patients. A recent example from the preclinical arena for precision medicine is hypoxia-activated prodrugs that only show efficacy in hypoxic tumours [30]. Molecular imaging using hypoxia PET tracers could allow identification of those patients who would benefit from these drugs. This preselection could be a necessity for targeted drugs that might be too expensive to prescribe without knowing the efficacy in the individual patient.

A possible downside of this approach is that because the biology of tumours is complex it might be difficult to select the appropriate imaging technique to define the optimal treatment. As mentioned above, tumour cells will typically develop resistance against a single treatment and different pathways will be activated.

\section{The future of imaging for precision medicine}

The two strategies above both have their advantages and disadvantages and are most likely complementary. Both require reliable and more robust imaging biomarkers, standardization, validated endpoints, genetic information, and more sophisticated trial designs. An important question will be how to implement these more advanced imaging approaches in clinical routine taking into account their availability and logistic considerations. Patient stratification and treatment monitoring will certainly be crucial steps for precision medicine in which imaging might play a major role.

\section{Acknowledgements}

The authors received research grants from the Seventh Framework Programme of the European Union (project ARTFORCE: 257144). 


\section{References}

1. Leibfarth, S., Simoncic, U., Mönnich, D., et al. Analysis of pairwise correlations in multi-parametric PET/MR data for biological tumor characterization and treatment individualization strategies. Eur J Nucl Med Mol Imaging 43, 1199-208 (2016).

2. Sauter, A. W., Schwenzer, N., Divine, M. R., Pichler, B. J. \& Pfannenberg, C. Image-derived biomarkers and multimodal imaging strategies for lung cancer management. Eur J Nucl Med Mol Imaging 42, 634-43 (2015).

3. Corroyer-Dulmont, A., Pérès, E. A., Gérault, A. N., et al. Multimodal imaging based on MRI and PET reveals $\left[{ }^{18}\right.$ F]FLT PET as a specific and early indicator of treatment efficacy in a preclinical model of recurrent glioblastoma. Eur J Nucl Med Mol Imaging 43, 682-94 (2016).

4. Henriksen, O. M., Larsen, V. A., Muhic, A., et al. Simultaneous evaluation of brain tumour metabolism, structure and blood volume using $\left[{ }^{18} \mathrm{~F}\right]$-fluoroethyltyrosine (FET) PET/MRI: feasibility, agreement and initial experience. Eur J Nucl Med Mol Imaging 43, 103-12 (2016).

5. Van Elmpt, W., Zegers, C. M. L., Reymen, B., et al. Multiparametric imaging of patient and tumour heterogeneity in non-small-cell lung cancer: quantification of tumour hypoxia, metabolism and perfusion. Eur J Nucl Med Mol Imaging 43, 240-8 (2016).

6. Gatidis, S., Schmidt, H., Martirosian, P. \& Schwenzer, N. F. Development of an MRI phantom for diffusion-weighted imaging with independent adjustment of apparent diffusion coefficient values and T2 relaxation times. Magn Reson Med 72, 459-63 (2014).

7. Boellaard, R., Delgado-Bolton, R., Oyen, W. J. G., et al. FDG PET/CT: EANM procedure guidelines for tumour imaging: version 2.0. Eur J Nucl Med Mol Imaging 42, 328-54 (2015).

8. Yankeelov, T. E., Mankoff, D. A., Schwartz, L. H., et al. Quantitative imaging in cancer clinical trials. Clin Cancer Res 22, 284-90 (2016).

9. Baas, P., Fennell, D., Kerr, K. M., Van Schil, P. E., Haas, R. L. \& Peters, S. Malignant pleural mesothelioma: ESMO clinical practice guidelines for diagnosis, treatment and follow-up. Ann Oncol 26 Suppl 5, v31-9 (2015).

10. Parker, C., Gillessen, S., Heidenreich, A. \& Horwich, A. Cancer of the prostate: ESMO clinical practice guidelines for diagnosis, treatment and follow-up. Ann Oncol 26 Suppl 5, v69-77 (2015). 
11. Morigi, J. J., Stricker, P. D., van Leeuwen, P. J., et al. Prospective comparison of ${ }^{18} \mathrm{~F}$ Fluoromethylcholine versus ${ }^{68} \mathrm{Ga}$-PSMA PET/CT in prostate cancer patients who have rising PSA after curative treatment and are being considered for targeted therapy. J Nucl Med 56, 1185-90 (2015).

12. Iqbal, R., Kramer, G. M., Verwer, E. E., et al. Multiparametric analysis of the relationship between tumor hypoxia and perfusion with ${ }^{18} \mathrm{~F}$-fluoroazomycin arabinoside and ${ }^{15} \mathrm{O}-\mathrm{H}_{2} \mathrm{O}$ PET. J Nucl Med 57, 530-5 (2016).

13. Bradshaw, T. J., Bowen, S. R., Jallow, N., Forrest, L. J. \& Jeraj, R. Heterogeneity in intratumor correlations of ${ }^{18} \mathrm{~F}$-FDG, ${ }^{18} \mathrm{~F}$-FLT, and ${ }^{61} \mathrm{Cu}$-ATSM PET in canine sinonasal tumors. J Nucl Med 54, 1931-7 (2013).

14. Metz, S., Ganter, C., Lorenzen, S., et al. Multiparametric MR and PET imaging of intratumoral biological heterogeneity in patients with metastatic lung cancer using voxel-by-voxel analysis. PLoS One 10, e0132386 (2015).

15. Aerts, H. J. W. L., van Baardwijk, A. A. W., Petit, S. F., et al. Identification of residual metabolic-active areas within individual NSCLC tumours using a preradiotherapy ${ }^{18}$ Fluorodeoxyglucose-PET-CT scan. Radiother Oncol 91, 386-92 (2009).

16. Due, A. K., Vogelius, I. R., Aznar, M. C., et al. Recurrences after intensity modulated radiotherapy for head and neck squamous cell carcinoma more likely to originate from regions with high baseline $\left[{ }^{18} \mathrm{~F}\right]-\mathrm{FDG}$ uptake. Radiother Oncol 111, 360-5 (2014).

17. Zschaeck, S., Haase, R., Abolmaali, N., et al. Spatial distribution of FMISO in head and neck squamous cell carcinomas during radio-chemotherapy and its correlation to pattern of failure. Acta Oncol 54, 1355-63 (2015).

18. Weber, D. C., Casanova, N., Zilli, T., et al. Recurrence pattern after $\left[{ }^{18} \mathrm{~F}\right]$ fluoroethyltyrosine-positron emission tomography-guided radiotherapy for high-grade glioma: a prospective study. Radiother Oncol 93, 586-92 (2009).

19. Dooms, C., van Baardwijk, A., Verbeken, E., et al. Association between ${ }^{18}$ F-fluoro2-deoxy-D-glucose uptake values and tumor vitality: prognostic value of positron emission tomography in early-stage non-small cell lung cancer. J Thorac Oncol 4, 822-8 (2009).

20. Van Baardwijk, A., Dooms, C., van Suylen, R. J., et al. The maximum uptake of ${ }^{18} \mathrm{~F}-$ deoxyglucose on positron emission tomography scan correlates with survival, hypoxia inducible factor-1alpha and GLUT-1 in non-small cell lung cancer. Eur J Cancer 43, 1392-8 (2007). 
21. Mazaheri, Y., Hricak, H., Fine, S. W., et al. Prostate tumor volume measurement with combined T2-weighted imaging and diffusion-weighted MR: correlation with pathologic tumor volume. Radiology 252, 449-57 (2009).

22. Puri, T., Chalkidou, A., Henley-Smith, R., et al. A method for accurate spatial registration of PET images and histopathology slices. EJNMMI Res 5, 64 (2015).

23. Christian, N., Lee, J. A., Bol, A., De Bast, M., Jordan, B. \& Grégoire, V. The limitation of PET imaging for biological adaptive-IMRT assessed in animal models. Radiother Oncol 91, 101-6 (2009).

24. Berwouts, D., Olteanu, L. A. M., Duprez, F, et al. Three-phase adaptive dosepainting-by-numbers for head-and-neck cancer: initial results of the phase I clinical trial. Radiother Oncol 107, 310-6 (2013).

25. Differding, S., Hanin, F. X. \& Grégoire, V. PET imaging biomarkers in head and neck cancer. Eur J Nucl Med Mol Imaging 42, 613-22 (2015).

26. Van Elmpt, W., De Ruysscher, D., van der Salm, A., et al. The PET-boost randomised phase II dose-escalation trial in non-small cell lung cancer. Radiother Oncol 104, 67-71 (2012).

27. De Bruin, E. C., McGranahan, N., Mitter, R., et al. Spatial and temporal diversity in genomic instability processes defines lung cancer evolution. Science 346, 251-6 (2014).

28. Cai, W., Lin, D., Wu, C., et al. Intratumoral heterogeneity of ALK-rearranged and ALK/EGFR coaltered lung adenocarcinoma. J Clin Oncol 33, 3701-9 (2015).

29. Bahce, I., Smit, E. F., Lubberink, M., et al. Development of $\left[{ }^{11} \mathrm{C}\right]$ erlotinib positron emission tomography for in vivo evaluation of EGF receptor mutational status. Clin Cancer Res 19, 183-93 (2013).

30. Peeters, S. G. J. A., Zegers, C. M. L., Biemans, R., et al. TH-302 in combination with radiotherapy enhances the therapeutic outcome and is associated with pretreatment $\left[{ }^{18} \mathrm{~F}\right] \mathrm{HX} 4$ hypoxia PET imaging. Clin Cancer Res 21, 2984-92 (2015). 



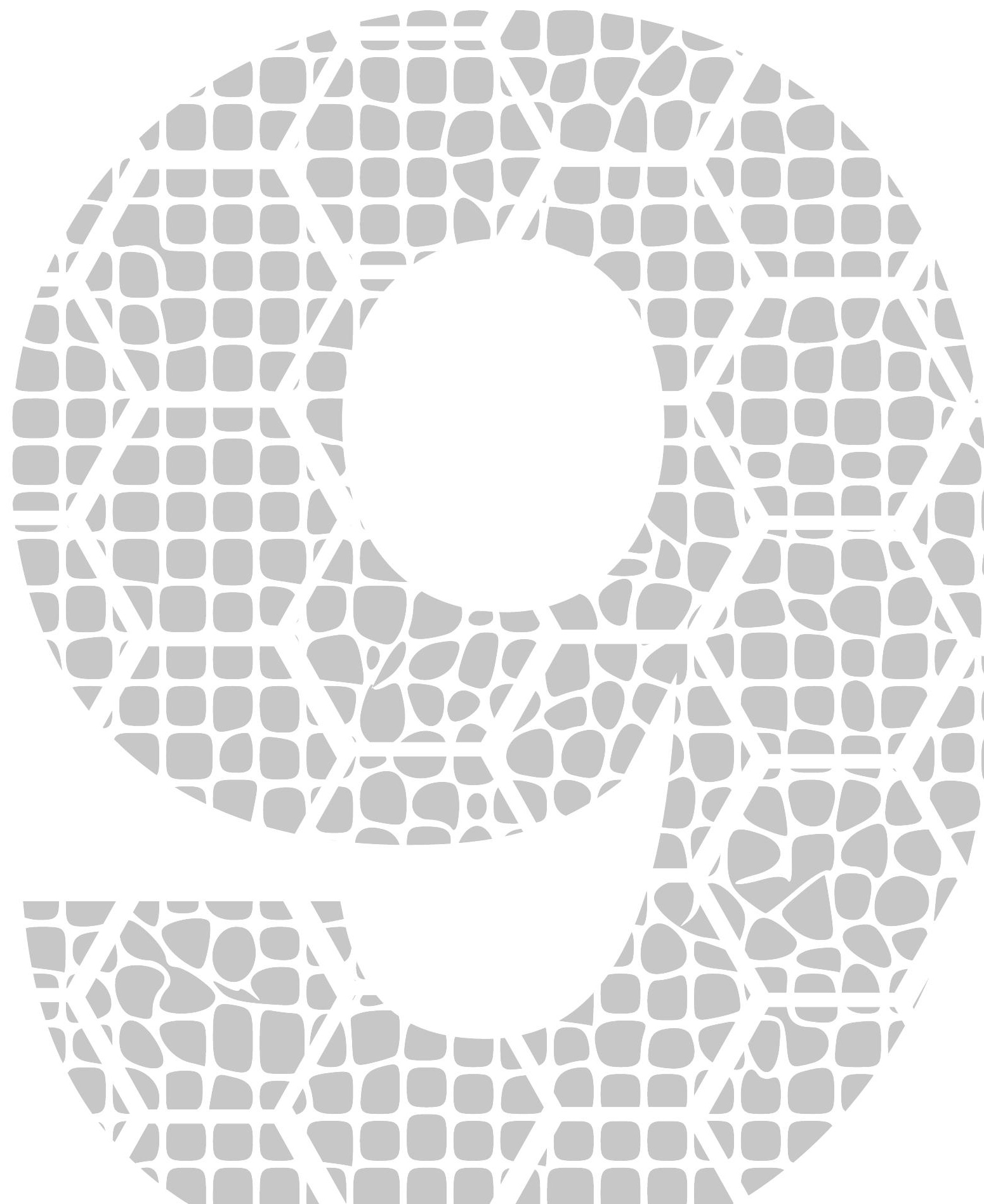




\section{Chapter 9}

General discussion and future perspectives 


\section{General discussion}

Despite substantial improvements in treatment options over the past decades, cancer remains one of the worldwide leading causes of mortality and morbidity. The prognosis between cancer types varies widely, with lung cancer as the number one cause of cancer related deaths [1]. To improve response rates, and to reduce unnecessary toxicity and costs, it is essential to quickly select the treatment with the highest expected benefit for the patient. Imaging can aid this treatment personalization by stratifying patients based on their prognosis and by predicting treatment efficacy. Additionally, imaging biomarkers can map tumour heterogeneities in 3D, identify resistant tumour areas, and guide treatment adaptation [2].

The research presented in this thesis aims to explore the use of functional imaging to characterize the tumour and tumour subvolumes, and subsequently to use these functional imaging characteristics for the prediction of patient prognosis, for patient stratification, and for treatment adaptation in non-small cell lung cancer (NSCLC), and head and neck cancer (HNC). We used various imaging modalities to visualize and quantify several features related to the different hallmarks of cancer. Positron emission tomography (PET) based imaging was used to visualize drug uptake, hypoxia levels, and tumour metabolism. Dynamic contrast-enhanced CT (DCE-CT) was used to image tumour vasculature.

\section{Visualization of drug uptake for patient stratification}

Monoclonal antibodies (mAb) have the ability to bind to specific targets on the tumour with high specificity and affinity. Although they have the potential to target cancer cells very selectively, their clinical benefit widely varies between patients. Labelling the antibodies with long-lived radionuclides, such as Zirconium-89, can show the distribution of the antibody in the tumour on PET and is hypothesized to be related to treatment success [3].

We showed, in the first part of this thesis, the clinical feasibility of labelling the epidermal growth factor receptor (EGFR) targeting drug cetuximab, with Zirconium89 (Chapter 2). No additional toxicity was associated with administration of ${ }^{89} \mathrm{Zr}$ cetuximab compared to standard cetuximab. Of the two dose schedules tested, the lower dose of $60 \mathrm{MBq}{ }^{89} \mathrm{Zr}$-cetuximab was recommended for future studies as the higher dose (120 MBq) did not provide improved image contrast. The subsequent phase II clinical trial (Chapter 3), showed a large variation in ${ }^{89} \mathrm{Zr}$-cetuximab uptake between the 17 included head and neck cancer patients. A discrepancy was shown between EGFR expression and ${ }^{89} \mathrm{Zr}$-cetuximab, confirming previously reported results in mice [4]. We hypothesized that for cetuximab to be beneficial, sufficient targets 
should be present in the tumour (i.e. EGF receptors), and cetuximab should reach these targets. In tumours lacking EGFR expression, response to the targeted drug was unexpected, while in tumours with high EGFR expression, the accessibility of the tumour was expected to be an important factor in cetuximab effectiveness. As hypothesized, we observed a mix of low and high ${ }^{89} \mathrm{Zr}$-cetuximab uptake in patients with high EGFR expression. However, in one patient with a low EGFR expression, a remarkably high ${ }^{89} \mathrm{Zr}$-cetuximab uptake was observed. Unfortunately, we were unable to link treatment outcome to these observed differences in ${ }^{89} \mathrm{Zr}$-cetuximab PET uptake, due to the small heterogeneous patient group with heterogeneous treatments from four different treatment arms.

Until now, to our knowledge, only one other clinical trial has been performed with ${ }^{89} \mathrm{Zr}$-cetuximab. This study in colorectal cancer did attempt to correlate ${ }^{89} \mathrm{Zr}$ cetuximab uptake and outcome, and showed promising results, though in a very small patient cohort. They found a clinical benefit in four out of six patients with uptake of ${ }^{89} \mathrm{Zr}$-cetuximab in the tumour, while in three out of four patients without uptake progressive disease was observed [5].

${ }^{89} \mathrm{Zr}$ PET imaging is not limited to cetuximab, labelling of other monoclonal antibodies has been explored in several studies. The first feasibility study using Zirconium-89 labelled antibodies, was published in 2006 [6]. Since then, several other studies have looked into the feasibility, biodistribution, optimal dose, and best imaging time point, for different $\mathrm{mAb}$ and various tumour sites [7-14]. A few studies have attempted to correlate treatment outcome and ${ }^{89} \mathrm{Zr}-\mathrm{mAb}$ PET imaging. Bahce et al. showed a positive trend, although not significant, between ${ }^{89} \mathrm{Zr}$-bevacizumab uptake $\left(\mathrm{SUV}_{\text {peak }}\right)$ and overall survival and progression free survival in NSCLC patients treated with chemotherapy plus bevacizumab [15]. Oosting et al. found for ${ }^{89} \mathrm{Zr}$-bevacizumab in renal cell carcinoma, that high baseline $S_{U V} V_{\max }$ was associated with a longer time to progression [16]. A study of ${ }^{89} \mathrm{Zr}$-fresolimumab in high-grade glioma, showed good uptake of labelled antibody, however, since all patients experienced recurrences, PET uptake could not be related to clinical benefit and the study was closed early [17]. Gebhart et al. used combined ${ }^{89} \mathrm{Zr}$-trastuzumab and FDG PET/CT to predict outcome of HER2 positive breast patients. With the combined approach, they could accurately predict morphological response and stratify patients based on their time to treatment failure [18].

In addition to baseline ${ }^{89} \mathrm{Zr}-\mathrm{mAb}$ PET imaging, some studies have explored the possibility of imaging during or shortly after treatment, to quantify early response to mAb treatment. Gaykema et al. performed ${ }^{89} \mathrm{Zr}$-trastuzumab and ${ }^{89} \mathrm{Zr}$-bevacizumab PET imaging at baseline and after 15 days, in breast cancer patients [19]. Changes on ${ }^{89} \mathrm{Zr}$-trastuzumab were positively associated with the response on CT. A study with ${ }^{89} \mathrm{Zr}$-bevacizumab in neuroendocrine tumours also found a correlation between the 
change in ${ }^{89} \mathrm{Zr}$-bevacizumab, between baseline 2, and 12 weeks after treatment, and the response on CT [20]. Van Es et al. examined the potential of ${ }^{89} \mathrm{Zr}$-bevacizumab PET as early indicator of treatment efficiency, at 2 and 6 weeks after start treatment, in metastatic renal cell carcinoma [21]. All patients that completed treatment had stable disease, and thus the potential of ${ }^{89} \mathrm{Zr}$-bevacizumab could not be evaluated.

Overall, the majority of the initial clinical trials with ${ }^{89} \mathrm{Zr}-\mathrm{mAb}$ have shown the potential of (early) response prediction in several cancer sides. Most studies have found heterogeneous PET uptake between patients, which would be one of the essential preconditions for patient stratification [22]. The trials performed so far are limited in their size; the number of included patients ranges between 7 and 23, with one exceptionally large trial of 56 patients [18]. Further validation in larger clinical trials is required.

\section{Imaging of tumour hypoxia, vasculature, and metabolism}

Hypoxia has been recognized as an important target in oncology. Tumour cells are about three times as sensitive to radiation in a well oxygenated environment compared to in an anoxic environment, in cell culture [23]. The poor vasculature in hypoxic regions may also limit the distribution of chemotherapy, while at the same time hypoxia promotes a more aggressive and metastatic phenotype [24, 25]. In Chapter 4 , we first evaluated the prognostic value of PET imaging with the hypoxia tracer ${ }^{18} \mathrm{~F}$ HX4 in NSCLC. We found that patients with hypoxia at baseline had significantly worse overall survival compared to non-hypoxic tumours. This is the first study evaluating the prognostic value of HX4 PET. Previous studies with different PET tracers, have also found hypoxia to be a negative prognostic factor for survival in NSCLC. A significantly worse overall survival was found for patients with elevated ${ }^{18} \mathrm{~F}$ FAZA, ${ }^{62} \mathrm{Cu}$-ATSM, or ${ }^{18} \mathrm{~F}$-FETNIM PET uptake [26, 27]. Progression free survival was negatively correlated with high ${ }^{18} \mathrm{~F}$-FAZA, ${ }^{62} \mathrm{Cu}$-ATSM, or ${ }^{18} \mathrm{~F}$-MISO uptake [27-29]. Hypoxia is a characteristic of most solid tumours and has shown to be prognostic in other tumour sites, such as head and neck cancer and cervix cancer [30].

In Chapter 4, we tried to overcome tumour hypoxia by adding the vasodilating drug nitroglycerin to standard (chemo)radiotherapy. In a randomized phase II trial in NSCLC, nitroglycerin combined with chemotherapy showed a significant benefit over chemotherapy alone [31]. However this result could not be replicated in other trials combining nitroglycerin with chemotherapy or chemoradiotherapy [32-35]. None of the mentioned clinical trials used the baseline hypoxia status to select patients. This is a general problem with hypoxia targeting studies; as patient stratification is rare, the full potential of hypoxia targeting strategies are not fully explored [36]. We aimed to analyse the effect of nitroglycerin on tumour hypoxia and tumour vasculature for each 
individual patient, to ultimately predict the benefit of nitroglycerin on a patient level. We found a mixed response of increases and decreases in both hypoxia and blood flow and blood volume. A substantial decrease in hypoxia, as a results of the nitroglycerin patch, was only found in a small subset of patients which was not sufficient to analyse any improvement in outcome from nitroglycerin.

PET imaging with 2-nitroimidazoles based PET tracers (e.g. HX4, FMISO, FAZA) is the most widely studied method to visualize hypoxia. These PET tracers are, however, not widely available and can be expensive, cumbersome, and time-consuming to implement in routine. In Chapter 6, we studied the feasibility of modelling tumour hypoxia based on other, more widely available, imaging modalities. We could create virtual hypoxia PET images, by combining information from FDG PET/CT and DCECT scans. This technique may help stratify patients based on hypoxia status in absence of a hypoxia PET scan. The tumours were subdivided in small homogeneous patches, i.e. supervoxels, and for each subregion, hypoxia levels were calculated. This spatial distribution of hypoxia could, for example, also be used for dose painting. Although promising results were obtained, these models still require further validation in a larger patient cohort and preferable with different hypoxia PET tracers. In the best performing model, FDG PET appeared to be the most important predictor of hypoxia, while DCE-CT features only marginally increased the performance. The information on the tumour vasculature, in this study extracted from DCE-CT images, could possibly be gained from contrast CT scans. This should be investigated in further validation studies. To our knowledge, this study is the first fully data-driven approach to create virtual hypoxia maps. Other studies predicting hypoxia have been based on mathematical models, or a combination of mathematical models with anatomical input, for example from histology or MRI [37-39].

In Chapter 7, we explored an approach to combine multiple imaging modalities and to use the combined knowledge to assess patient prognosis. As discussed in Chapter 8, with the increasing availability of multiparametric imaging, there is an increasing need for analyses methods that can incorporate all available knowledge, and allow to assess multiple biological and functional properties simultaneously. We have developed such a technique and tested this on the same imaging set that was used as in Chapter 6, containing FDG PET/CT, hypoxia HX4 PET/CT and DCE-CT scans. An advantage of this technique is that uncertainties caused by the registration of the images or noise, are reduced by clustering similar voxels into supervoxels. The supervoxels of all tumours were grouped into four phenotypical clusters. The cluster with a high HX4 PET uptake, high FDG PET uptake, and intermediate blood flow and blood volume, was predictive for overall survival. Patients with a substantially high volume of this supervoxel cluster had a significantly lower overall survival compared to patients with a low relative or absolute volume of the cluster. Wu et al. performed 
a similar analysis in NSCLC, but only for CT and FDG PET imaging, and also found that the most metabolically active and metabolically heterogeneous subvolumes were prognostic for overall survival [40].

\section{Functional imaging for treatment adaptation — dose painting}

Dose painting is an attractive method to integrate tumour biology into radiotherapy treatment planning. It is likely that tumour heterogeneity affects radioresistance. If given a uniform dose throughout the tumour, local recurrences have shown to arise from small radioresistant niches $[41,42]$. Increasing the total radiotherapy dose to the tumour could theoretically increase the tumour control probability and is the safest approach if no information on radioresistance is available, however, the dose escalation that can be achieved is often limited by the healthy organs surrounding the tumour. In NSCLC it was shown that total tumour dose escalation without proper patient selection could even be harmful [43].

Dose painting requires an imaging surrogate to map the radioresistant tumour regions. Which imaging surrogate correlates best with radioresistance, and would therefore be best suitable for dose escalation, is still a matter of debate. Several imaging features linked to one of the hallmarks of cancer have been proposed. In Chapter 5, we compared for NSCLC, uniform dose escalation to the whole tumour, with boosting based on FDG PET and hypoxia HX4 PET, and found that a significantly higher dose could be achieved for the PET-based dose painting approaches, without increasing the dose to the organs at risk. The highest planned doses were feasible for the hypoxia PET plans, which had the smallest boost volumes. The FDG PET dose painting and uniform boosting are currently under evaluation in the prospective randomized PET-boost trial (NCT01024829) [44]. Results are expected around October 2018. The phase II clinical trial contained an extensive imaging protocol of FDG PET/CT, hypoxia HX4 PET/CT and DCE-CT. These images were also used for the analyses performed in Chapter 6 and Chapter 7.

In NSCLC, several other trials have evaluated dose painting or are currently studying the approach. Wanet et al. performed a single arm feasibility study of FDG PET boosting in 13 patients. In peripheral tumours, they could achieve an average dose of $89.2 \mathrm{~Gy}$ at acceptable toxicity rates. In central tumours, however, they observed severe toxicity at an average dose of $75 \mathrm{~Gy}$ [45]. Vera et al. recently reported their results on a hypoxia dose painting trial in locally advanced NSCLC [29]. Patients without hypoxic tumours received a standard dose of 66 Gy; patients with baseline hypoxia on FMISO PET scans received an extra boost, where the extra boost was limited by the dose constraints of the lungs and spinal cord. They found that baseline hypoxia had a strong negative effect on patient prognosis, but they could not reverse this effect by 
increasing the radiotherapy dose to these areas. It has to be noted though, that out of the 34 patients in the experimental arm, only 7 patients received a maximum dose of 80-86 Gy, 17 patients received a dose between 70 and 76 Gy, and for 10 patients, no dose escalation could be achieved. Several other studies have been initiated and are still ongoing. The multicentre phase III NARLAL2 trial (NCT02354274), is comparing FDG PET dose painting with standard 66 Gy in 33 fractions [46]. The randomized stage II-III RTEP7 trial (NCT02473133) is assessing dose redistribution based on FDG PET during treatment, after $42 \mathrm{~Gy}$ [47]. A similar approach is being tested in the randomized phase II RTOG-1106/ACRIN-6697 study (NCT01507428), where the plan is adapted after 18-19 fractions based on the FDG PET/CT [48]. Clinical results for all of these trials are awaited.

In head and neck cancer, the first clinical trial tested dose escalation in two dose levels (72.5 Gy and 77.5 Gy) to FDG-PET-positive subvolumes. One treatment related death in the second dose level halted the study [49]. A second study by the same group explored an adaptive dose painting by number approach on FDG PET voxel intensities combined with a conventional uniform dose. From the two dose levels tested, 80.9 Gy and $85.9 \mathrm{~Gy}$, significant late toxicities (i.e. mucosal ulcers) were observed, and 80.9 Gy was found to be the maximum tolerated dose [50]. Another phase I study tested uniform dose escalation of $82 \mathrm{~Gy}$ in the FDG high uptake region in 15 patients. The acute toxicities were within the protocol defined criteria, however, in two patients ulcerations did not heal and raised concerns regarding late toxicities [51]. Ongoing studies in head and neck cancer include a phase II randomized trial on FDG dose painting by numbers (NCT01341535) [52], a phase II trial comparing standard irradiation of 70 Gy with adaptive FDG PET dose redistribution of 64-84 Gy (NCT01504815) [53], and a phase II trial testing a $10 \%$ dose increase in hypoxic areas based on ${ }^{18} \mathrm{~F}$-MISO PET [54].

Dose painting strategies are also under investigation in other tumour sites, prostate [55, 56], or bone metastases [57] for example. The results of these studies, and the ongoing studies in NSCLC and HNC, should gain more insight into the benefits and limitations of dose painting.

\section{Future perspectives}

In this thesis, we showed some potential applications of integrating functional imaging in the radiotherapy workflow. It is expected that in future, the combination of more advanced and precise radiotherapy delivery techniques, sophisticated functional imaging methods, and improved validated biomarkers and models, will further enable treatment optimization and personalization. 


\section{Advances in radiotherapy techniques}

Radiotherapy techniques have made significant improvements over the last decades. Modern external beam irradiation is delivered with highly conformal photon beams in short treatment times. Small tumour (sub)volumes can be irradiated accurately, with high doses, which is exploited during dose painting. Modern clinical linear accelerators are, however, reaching their physical limit, and significant further improvements in dose distributions are unexpected $[58,59]$. Two exciting developments that are expected to substantially improve radiotherapy treatment for carefully selected patients, are the MRI-guided linear accelerator (MR-linac) and proton therapy [59]. These developments are currently under clinical evaluation and may be particularly important for a subset of NSCLC and head and cancer patients. The MR-linac is a hybrid modality, combining a linac with magnetic resonance imaging (MRI). The theoretical advantages of the MR-linac over cone-beam CT (CBCT) guided linacs, are the optimal soft tissue contrast imaging, the possibility to image during treatment, and image repeatedly without additional radiation dose. The system has the potential to reduce the uncertainties in tumour location, and enable fast replanning, gated irradiation, and tumour tracking. Consequently, the reduced uncertainties may allow for dose escalation or reduction in normal tissue toxicities, and adaptive focal boosting [60]. It remains to be seen if the uncertainty reduction from having the MRI images outweigh the increased spatial uncertainty of MRI compared to CT.

The other treatment technique currently installed or being installed in many centres in Europe and worldwide is proton therapy. Proton beams have the unique property to deposit almost all their energy at a particular depth, the so-called Bragg peak. Whereas photon treatments cause ionization damage over the whole beam length, proton beams release most of their energy towards the end of the beam and have a minimal exit dose. These favourable beam characteristics could theoretically target the tumour more accurately and reduce the dose to the organs at risk [61].

Both the MR-linac and proton therapy have the potential to deliver ionizing radiation with high accuracy. Combining functional imaging and these more accurate delivery techniques could optimize irradiating of biological target volumes. A main disadvantage of both technologies, compared to CBCT-guided photon treatment, is the increased costs. The clinical effectiveness and cost effectiveness are currently under evaluation [61-63]. Proper patient selection, e.g. based on (functional) imaging, is expected to be crucial to achieve the full potential of the MR-linac and proton therapy. 


\section{Developments in functional imaging and biomarkers}

Even though a variety of functional imaging techniques were explored in this thesis, including DCE-CT and several PET based approaches, this is only a small subset of the functional imaging techniques currently under clinical investigation [64]. In Chapter 8, we briefly mentioned some other functional imaging modalities, for example, ${ }^{18} \mathrm{~F}$ FLT PET, a biomarker for proliferation, or labelled drugs, such as, ${ }^{68} \mathrm{Ga}-\mathrm{PSMA}$ or ${ }^{89} \mathrm{Zr}$-bevacizumab. In this thesis, the PET tracer HX4 was used for hypoxia imaging. Several other ${ }^{18} \mathrm{~F}$ labelled 2-nitroimidazole PET tracers are currently under evaluation, that all have the same working principle, but have slightly different characteristics [65]. Other, more indirect markers for hypoxia, that have been examined include DCE-CT, ${ }^{15} \mathrm{O}$-labelled water, a variety of SPECT markers, and several MRI techniques, e.g. blood oxygen level dependent (BOLD), diffusion-weighted imaging (DWI), and oxygen-enhanced MRI [30, 36].

Despite the numerous imaging biomarkers used in clinical research, including many showing great potential, only a few make the transition into clinical practise. Crossing this translational gap will be one of the major challenges in imaging research. Before biomarkers can be approved for clinical decision making, biomarkers need to be reproducible (in a multicentre setting), biologically and clinically validated, and cost-effective [2,66]. Another challenge, as a consequence of the wide availability of different imaging modalities and the availability of multiparametric imaging, is the challenge of combining information from multiple imaging sources. Two approaches to deal with this were discussed in Chapter 8 .

In parallel to the developments in functional imaging techniques, more advanced imaging analysis methods and big data approaches are gaining research interest. These methods use tumour features based on image intensity, shape, and texture for example, or deep learning approaches [67,68]. Advanced image analysis methods have the potential to improve predictive models and help patient stratification.

To conclude, radiotherapy delivery techniques and functional imaging modalities are developing at a fast pace. The combination of very accurate radiation delivery modalities with biologically validated targets, will allow further optimization and personalization of treatment to increase the tumour control and reduce normal tissue toxicities. 


\section{References}

1. Ferlay, J., Soerjomataram, I., Ervik, M., et al. GLOBOCAN 2012 v1.0, cancer incidence and mortality worldwide: IARC cancerbase no. $11 \mathrm{http}$ //globocan.iarc. fr.

2. O’Connor, J. P. B., Aboagye, E. O., Adams, J. E., et al. Imaging biomarker roadmap for cancer studies. Nat Rev Clin Oncol 14, 169-86 (2017).

3. Wright, B. D. \& Lapi, S. E. Designing the magic bullet? The advancement of immuno-PET into clinical use. J Nucl Med 54, 1171-4 (2013).

4. Aerts, H. J. W. L., Dubois, L., Perk, L., et al. Disparity between in vivo EGFR expression and ${ }^{89} \mathrm{Zr}$-labeled cetuximab uptake assessed with PET. J Nucl Med 50, 123-31 (2009).

5. Menke-van der Houven van Oordt, C. W., Gootjes, E. C., Huisman, M. C., et al. ${ }^{89} \mathrm{Zr}$-cetuximab PET imaging in patients with advanced colorectal cancer. Oncotarget 6, 30384-93 (2015).

6. Borjesson, P. K. E., Jauw, Y. W., Boellaard, R., et al. Performance of immunopositron emission tomography with zirconium-89-labeled chimeric monoclonal antibody U36 in the detection of lymph node metastases in head and neck cancer patients. Clin Cancer Res 12, 2133-40 (2006).

7. Dijkers, E. C., Oude Munnink, T. H., Kosterink, J. G., et al. Biodistribution of ${ }^{89} \mathrm{Zr}$-trastuzumab and PET imaging of HER2-positive lesions in patients with metastatic breast cancer. Clin Pharmacol Ther 87, 586-92 (2010).

8. Gaykema, S. B. M., Brouwers, A. H., Lub-de Hooge, M. N., et al. ${ }^{89}$ Zr-bevacizumab PET imaging in primary breast cancer. J Nucl Med 54, 1014-8 (2013).

9. Pandit-Taskar, N., O’Donoghue, J. A., Durack, J. C., et al. A phase I/II study for analytic validation of ${ }^{89} \mathrm{Zr}$-J591 immunoPET as a molecular imaging agent for metastatic prostate cancer. Clin Cancer Res 21, 5277-85 (2015).

10. Lamberts, L. E., Menke-van der Houven van Oordt, C. W., ter Weele, E. J., et al. ImmunoPET with anti-mesothelin antibody in patients with pancreatic and ovarian cancer before anti-mesothelin antibody-drug conjugate treatment. Clin Cancer Res 22, 1642-52 (2016).

11. Ulaner, G. A., Hyman, D. M., Ross, D. S., et al. Detection of HER2-positive metastases in patients with HER2-negative primary breast cancer using ${ }^{89} \mathrm{Zr}$ trastuzumab PET/CT. J Nucl Med 57, 1523-8 (2016).

12. Bensch, F., Lamberts, L. E., Smeenk, M. M., et al. ${ }^{89}$ Zr-lumretuzumab PET imaging before and during HER3 antibody lumretuzumab treatment in patients with solid tumors. Clin Cancer Res (2017). 
13. Jansen, M. H., Veldhuijzen van Zanten, S. E. M., van Vuurden, D. G., et al. Molecular drug imaging: ${ }^{89} \mathrm{Zr}$-bevacizumab PET in children with diffuse intrinsic pontine glioma. J Nucl Med 58, 711-6 (2017).

14. O’Donoghue, J. A., Lewis, J. S., Pandit-Taskar, N., et al. Pharmacokinetics, biodistribution, and radiation dosimetry for ${ }^{89} \mathrm{Zr}$-trastuzumab in patients with esophagogastric cancer. J Nucl Med (2017).

15. Bahce, I., Huisman, M. C., Verwer, E. E., et al. Pilot study of ${ }^{89} \mathrm{Zr}$-bevacizumab positron emission tomography in patients with advanced non-small cell lung cancer. EJNMMI Res 4, 35 (2014).

16. Oosting, S. F., Brouwers, A. H., van Es, S. C., et al. ${ }^{89}$ Zr-bevacizumab PET visualizes heterogeneous tracer accumulation in tumor lesions of renal cell carcinoma patients and differential effects of antiangiogenic treatment. J Nucl Med 56, 63-9 (2015).

17. Den Hollander, M. W., Bensch, F., Glaudemans, A. W. J. M., et al. TGF-beta antibody uptake in recurrent high-grade glioma imaged with ${ }^{89} \mathrm{Zr}$-fresolimumab PET. J Nucl Med 56, 1310-4 (2015).

18. Gebhart, G., Lamberts, L. E., Wimana, Z., et al. Molecular imaging as a tool to investigate heterogeneity of advanced HER2-positive breast cancer and to predict patient outcome under trastuzumab emtansine (T-DM1): the ZEPHIR trial. Ann Oncol 27, 619-24 (2016).

19. Gaykema, S. B. M., Schröder, C. P., Vitfell-Rasmussen, J., et al. ${ }^{89} \mathrm{Zr}$-trastuzumab and ${ }^{89} \mathrm{Zr}$-bevacizumab PET to evaluate the effect of the HSP90 inhibitor NVPAUY922 in metastatic breast cancer patients. Clin Cancer Res 20, 3945-54 (2014).

20. Van Asselt, S. J., Oosting, S. F., Brouwers, A. H., et al. Everolimus reduces ${ }^{89} \mathrm{Zr}-$ bevacizumab tumor uptake in patients with neuroendocrine tumors. J Nucl Med 55, 1087-92 (2014).

21. Van Es, S. C., Brouwers, A. H., Mahesh, S. V. K., et al. ${ }^{89} \mathrm{Zr-bevacizumab} \mathrm{PET:}$ potential early indicator of everolimus efficacy in patients with metastatic renal cell carcinoma. J Nucl Med 58, 905-10 (2017).

22. Jauw, Y. W. S., Menke-van der Houven van Oordt, C. W., Hoekstra, O. S., et al. Immuno-positron emission tomography with zirconium-89-labeled monoclonal antibodies in oncology: what can we learn from initial clinical trials? Front Pharmacol 7, 131 (2016).

23. Gray, L. H., Conger, A. D., Ebert, M., Hornsey, S. \& Scott, O. C. A. The concentration of oxygen dissolved in tissues at the time of irradiation as a factor in radiotherapy. Br J Radiol 26, 638-48 (1953). 
24. Wouters, B. G., van den Beucken, T., Magagnin, M. G., Lambin, P. \& Koumenis, C. Targeting hypoxia tolerance in cancer. Drug Resist Updat 7, 25-40 (2004).

25. Wilson, W. R. \& Hay, M. P. Targeting hypoxia in cancer therapy. Nat Rev Cancer 11, 393-410 (2011).

26. Li, L., Hu, M., Zhu, H., Zhao, W., Yang, G. \& Yu, J. Comparison of $\left[{ }^{18} \mathrm{~F}\right]-$ fluoroerythronitroimidazole and $\left[{ }^{18} \mathrm{~F}\right]$-fluorodeoxyglucose positron emission tomography and prognostic value in locally advanced non-small-cell lung cancer. Clin Lung Cancer 11, 335-40 (2010).

27. Kinoshita, T., Fujii, H., Hayashi, Y., Kamiyama, I., Ohtsuka, T. \& Asamura, H. Prognostic significance of hypoxic PET using ${ }^{18} \mathrm{~F}$-FAZA and ${ }^{62} \mathrm{Cu}$-ATSM in nonsmall-cell lung cancer. Lung Cancer 91, 56-66 (2016).

28. Eschmann, S. M., Paulsen, F., Reimold, M., et al. Prognostic impact of hypoxia imaging with ${ }^{18} \mathrm{~F}$-misonidazole PET in non-small cell lung cancer and head and neck cancer before radiotherapy. J Nucl Med 46, 253-60 (2005).

29. Vera, P., Thureau, S., Chaumet-Riffaud, P., et al. Phase II study of a radiotherapy total dose increase in hypoxic lesions identified by F-miso PET/CT in patients with non-small cell lung carcinoma [RTEP5 study]. J Nucl Med 58, 1045-53 (2017).

30. Horsman, M. R., Mortensen, L. S., Petersen, J. B., Busk, M. \& Overgaard, J. Imaging hypoxia to improve radiotherapy outcome. Nat Rev Clin Oncol 9, 674-87 (2012).

31. Yasuda, H., Yamaya, M., Nakayama, K., et al. Randomized phase II trial comparing nitroglycerin plus vinorelbine and cisplatin with vinorelbine and cisplatin alone in previously untreated stage IIIB/IV non-small-cell lung cancer. J Clin Oncol 24, 688-94 (2006).

32. Arrieta, O., Blake, M., de la Mata-Moya, M. D., et al. Phase II study. Concurrent chemotherapy and radiotherapy with nitroglycerin in locally advanced nonsmall cell lung cancer. Radiother Oncol 111, 311-5 (2014).

33. Reinmuth, N., Meyer, A., Hartwigsen, D., et al. Randomized, double-blind phase II study to compare nitroglycerin plus oral vinorelbine plus cisplatin with oral vinorelbine plus cisplatin alone in patients with stage IIIB/IV non-small cell lung cancer (NSCLC). Lung Cancer 83, 363-8 (2014).

34. Davidson, A., Veillard, A. S., Tognela, A., et al. A phase III randomized trial of adding topical nitroglycerin to first-line chemotherapy for advanced nonsmallcell lung cancer: the Australasian lung cancer trials group NITRO trial. Ann Oncol 26, 2280-6 (2015). 
35. Dingemans, A. M. C., Groen, H. J. M., Herder, G. J. M., et al. A randomized phase II study comparing paclitaxel-carboplatin-bevacizumab with or without nitroglycerin patches in patients with stage IV nonsquamous nonsmall-cell lung cancer: NVALT12 (NCT01171170). Ann Oncol 26, 2286-93 (2015).

36. Dewhirst, M. W. \& Birer, S. R. Oxygen-enhanced MRI is a major advance in tumor hypoxia imaging. Cancer Res 76, 769-72 (2016).

37. Kelly, C. J. \& Brady, M. A model to simulate tumour oxygenation and dynamic $\left[{ }^{18} \mathrm{~F}\right]$-Fmiso PET data. Phys Med Biol 51, 5859-73 (2006).

38. Gu, S., Chakraborty, G., Champley, K., et al. Applying a patient-specific biomathematical model of glioma growth to develop virtual $\left[{ }^{18} \mathrm{~F}\right]$-FMISO-PET images. Math Med Biol 29, 31-48 (2012).

39. Grimes, D. R., Kannan, P., Warren, D. R., et al. Estimating oxygen distribution from vasculature in three-dimensional tumour tissue. J R Soc Interface 13, 20160070 (2016).

40. Wu, J., Gensheimer, M. F., Dong, X., et al. Robust intratumor partitioning to identify high-risk subregions in lung cancer: a pilot study. Int J Radiat Oncol Biol Phys 95, 1504-12 (2016).

41. Aerts, H. J. W. L., van Baardwijk, A. A. W., Petit, S. F., et al. Identification of residual metabolic-active areas within individual NSCLC tumours using a preradiotherapy ${ }^{18}$ Fluorodeoxyglucose-PET-CT scan. Radiother Oncol 91, 386-92 (2009).

42. Due, A. K., Vogelius, I. R., Aznar, M. C., et al. Recurrences after intensity modulated radiotherapy for head and neck squamous cell carcinoma more likely to originate from regions with high baseline $\left[{ }^{18} \mathrm{~F}\right]$-FDG uptake. Radiother Oncol 111, 360-5 (2014).

43. Bradley, J. D., Paulus, R., Komaki, R., et al. Standard-dose versus high-dose conformal radiotherapy with concurrent and consolidation carboplatin plus paclitaxel with or without cetuximab for patients with stage IIIA or IIIB nonsmall-cell lung cancer (RTOG 0617): a randomised, two-by-two factorial phase 3 study. Lancet Oncol 16, 187-99 (2015).

44. Van Elmpt, W., De Ruysscher, D., van der Salm, A., et al. The PET-boost randomised phase II dose-escalation trial in non-small cell lung cancer. Radiother Oncol 104, 67-71 (2012).

45. Wanet, M., Delor, A., Hanin, F. X., et al. An individualized radiation dose escalation trial in non-small cell lung cancer based on FDG-PET imaging. Strahlenther Onkol (2017). 
46. Møller, D. S., Nielsen, T. B., Brink, C., et al. Heterogeneous FDG-guided doseescalation for locally advanced NSCLC (the NARLAL2 trial): design and early dosimetric results of a randomized, multi-centre phase-III study. Radiother Oncol 124, 311-7 (2017).

47. U.S. National Institutes of Health. ClinicalTrials.gov Study of interest of personalized radiotherapy dose redistribution in patients with stage III NSCLC (RTEP7). https://clinicaltrials.gov/ct2/show/NCT02473133.

48. U.S. National Institutes of Health. ClinicalTrials.gov Study of positron emission tomography and computed tomography in guiding radiation therapy in patients with stage III non-small cell lung cancer. https: / / clinicaltrials.gov / ct2 / show / NCT01507428.

49. Madani, I., Duthoy, W., Derie, C., et al. Positron Emission Tomography-guided, focal-dose escalation using intensity-modulated radiotherapy for head and neck cancer. Int J Radiat Oncol Biol Phys 68, 126-35 (2007).

50. Madani, I., Duprez, F., Boterberg, T., et al. Maximum tolerated dose in a phase I trial on adaptive dose painting by numbers for head and neck cancer. Radiother Oncol 101, 351-5 (2011).

51. Rasmussen, J. H., Håkansson, K., Vogelius, I. R., et al. Phase I trial of ${ }^{18} \mathrm{~F}$ Fludeoxyglucose based radiation dose painting with concomitant cisplatin in head and neck cancer. Radiother Oncol 120, 76-80 (2016).

52. U.S. National Institutes of Health. ClinicalTrials.gov A study comparing adaptive biological imaging - voxel intensity - based radiotherapy (adaptive dose escalation) versus standard radiotherapy for head and neck cancer. https : / / clinicaltrials.gov/ct2/show/NCT01341535.

53. Heukelom, J., Hamming, O., Bartelink, H., et al. Adaptive and innovative Radiation Treatment FOR improving Cancer treatment outcomE (ARTFORCE); a randomized controlled phase II trial for individualized treatment of head and neck cancer. BMC Cancer 13, 84 (2013).

54. Welz, S., Mönnich, D., Pfannenberg, C., et al. Prognostic value of dynamic hypoxia PET in head and neck cancer: Results from a planned interim analysis of a randomized phase II hypoxia-image guided dose escalation trial. Radiother Oncol (2017).

55. U.S. National Institutes of Health. ClinicalTrials.gov Hypofractionated imageguided radiotherapy for prostate cancer: The HEIGHT trial (HEIGHT). https: / / clinicaltrials.gov/ct2/show/NCT01411332. 
56. U.S. National Institutes of Health. ClinicalTrials.gov BIOPROP20: biologically optimised IMRT for prostate radiotherapy hypofractionated radiotherapy with intra-prostatic boosts to tumour nodules in men with intermediate and high risk prostate cancer (BIOPROP20). https : / / clinicaltrials . gov / ct2 / show / NCT02125175.

57. U.S. National Institutes of Health. ClinicalTrials.gov Biological image guided antalgic stereotactic body radiotherapy of bone metastases. https://clinicaltrials. gov/ct2/show/NCT01429493.

58. Gregoire, V., Langendijk, J. A. \& Nuyts, S. Advances in radiotherapy for head and neck cancer. J Clin Oncol 33, 3277-84 (2015).

59. Baumann, M., Krause, M., Overgaard, J., et al. Radiation oncology in the era of precision medicine. Nat Rev Cancer 16, 234-49 (2016).

60. Kerkmeijer, L. G. W., Fuller, C. D., Verkooijen, H. M., et al. The MRI-linear accelerator consortium: evidence-based clinical introduction of an innovation in radiation oncology connecting researchers, methodology, data collection, quality assurance, and technical development. Front Oncol 6, 215 (2016).

61. Chang, J. Y., Jabbour, S. K., De Ruysscher, D., et al. Consensus statement on proton therapy in early-stage and locally advanced non-small cell lung cancer. Int J Radiat Oncol Biol Phys 95, 505-16 (2016).

62. Langendijk, J. A., Lambin, P., De Ruysscher, D., Widder, J., Bos, M. \& Verheij, M. Selection of patients for radiotherapy with protons aiming at reduction of side effects: the model-based approach. Radiother Oncol 107, 267-73 (2013).

63. Verma, V., Mishra, M. V. \& Mehta, M. P. A systematic review of the cost and costeffectiveness studies of proton radiotherapy. Cancer 122, 1483-501 (2016).

64. Aboagye, E. O. \& Kraeber-Bodere, F. Highlights lecture EANM 2016: "Embracing molecular imaging and multi-modal imaging: a smart move for nuclear medicine towards personalized medicine”. Eur J Nucl Med Mol Imaging 44, 1559-74 (2017).

65. Peeters, S. G. J. A., Zegers, C. M. L., Lieuwes, N. G., et al. A comparative study of the hypoxia PET tracers $\left[{ }^{18} \mathrm{~F}\right]-\mathrm{HX} 4,\left[{ }^{18} \mathrm{~F}\right]-\mathrm{FAZA}$, and $\left[{ }^{18} \mathrm{~F}\right]-\mathrm{FMISO}$ in a preclinical tumor model. Int J Radiat Oncol Biol Phys 91, 351-9 (2015).

66. Aide, N., Lasnon, C., Veit-Haibach, P., Sera, T., Sattler, B. \& Boellaard, R. EANM/EARL harmonization strategies in PET quantification: from daily practice to multicentre oncological studies. Eur J Nucl Med Mol Imaging 44, 17-31 (2017).

67. Aerts, H. J. W. L., Velazquez, E. R., Leijenaar, R. T. H., et al. Decoding tumour phenotype by noninvasive imaging using a quantitative radiomics approach. Nat Commun 5, 4006 (2014). 
68. Litjens, G., Kooi, T., Bejnordi, B. E., et al. A survey on deep learning in medical image analysis. Med Image Anal 42, 60-88 (2017). 



\section{List of abbreviations}


$\mathrm{BF}$

BV

CI

CR

CT

CTV

$\mathrm{D}_{0.1}$

DCE-CT

$\mathrm{D}_{\text {mean }}$

DNA

DVH

EGFR

EQD2

${ }^{18} \mathrm{~F}$

${ }^{18}$ F-FDG

${ }^{18} \mathrm{~F}-\mathrm{HX} 4$

FHV

GTV

$\mathrm{GTV}_{\ln }$

$\mathrm{GTV}_{\text {prim }}$

Gy

$\mathrm{HF}$

HNC

HNSCC

HU

HV

IHC

IQR
Blood flow

Blood volume

Confidence interval

Coefficient of repeatability

Computed tomography

Clinical target volume

Dose received by maximal $0.1 \%$ of the organ

Dynamic contrast-enhanced CT

Mean dose

Deoxyribonucleic acid

Dose-volume histogram

Epidermal growth factor receptor

Equivalent dose in 2 Gy fractions

Fluorine-18

Fluorodeoxyglucose

Fluortanidazole

Fraction of hypoxic volume

Gross tumour volume

GTV of the involved lymph nodes

GTV of the primary tumour

Gray

Hypoxic fraction

Head and neck cancer

Head and neck squamous cell carcinoma

Hounsfield unit

Hypoxic volume

Immunohistochemistry

Interquartile range 


\begin{tabular}{|c|c|}
\hline LAHNSCC & Locally advanced head and neck squamous cell carcinomas \\
\hline LRPFS & Locoregional progression-free survival \\
\hline $\mathrm{mAb}$ & Monoclonal antibody \\
\hline $\mathrm{MBq}$ & Megabecquerel \\
\hline MFS & Metastasis-free survival \\
\hline MRI & Magnetic resonance imaging \\
\hline NSCLC & Non-small cell lung cancer \\
\hline OAR & Organs at risk \\
\hline $\mathrm{OF}$ & Overlap fraction \\
\hline OS & Overall survival \\
\hline PET & Positron emission tomography \\
\hline PRV & Planning risk volume \\
\hline PTV & Planning target volume \\
\hline RMSE & Root-mean-square error \\
\hline ROI & Region of interest \\
\hline SD & Standard deviation \\
\hline SNR & Signal-to-noise ratio \\
\hline SPECT & Single-photon emission computed tomography \\
\hline SUV & Standardized uptake value \\
\hline $\mathrm{SUV}_{\max }$ & Maximum standardized uptake value \\
\hline $\mathrm{SUV}_{\text {mean }}$ & Mean standardized uptake value \\
\hline$S_{U V}$ peak & Peak standardized uptake value \\
\hline Sv & Sievert \\
\hline TBR & Tumour-to-background ratio \\
\hline $\mathrm{V}_{36}$ & Volume receiving at least $36 \mathrm{~Gy}$ \\
\hline VMAT & Volumetric arc therapy \\
\hline WHO & World Health Organization \\
\hline${ }^{89} \mathrm{Zr}$ & Zirconium-89 \\
\hline
\end{tabular}


Summary 


\section{Background and aim of the thesis}

Cancer is a worldwide health problem with over 14 million new cases in 2012. The number of new cancer cases is expected to increase and is estimated to reach almost 24 million by 2035. Despite substantial advances in treatment options, survival for many cancer types remains poor. Treatment is challenging due to the similarity between cancerous and healthy cells, and due to the heterogeneity of the disease.

To improve patient survival, and to reduce unnecessary toxicity and cost, it is essential to quickly select the treatment with the highest expected benefit. Functional imaging has the potential to guide treatment personalization by stratifying patients based on their prognosis and on the predicted treatment benefit. Functional imaging biomarkers can map tumour heterogeneities, identify resistant tumour regions, and guide treatment adaptation.

In this thesis, the use of functional imaging to characterize the tumour and tumour subvolumes was explored. Positron emission tomography (PET) was used to image drug uptake, hypoxia levels, and tumour metabolism. Dynamic contrast-enhanced CT (DCE-CT) was used to image tumour vasculature. Subsequently, these functional imaging characteristics were tested as prognostic markers, for patient stratification, and for treatment adaptation in non-small cell lung cancer and head and neck cancer.

\section{Non-invasive imaging of drug uptake}

In the first part of this thesis, non-invasive visualization and quantification of the monoclonal antibody cetuximab was explored. Monoclonal antibodies have the capability to bind to their targets with high specificity and affinity. Cetuximab, for example, targets the epidermal growth factor receptor (EGFR). The clinical benefit of monoclonal antibodies depend on the expression of the target in the tumour and on how well the antibody can reach these targets. We investigated the accessibility of the tumour to cetuximab with PET, by labelling cetuximab with radioactive Zirconium-89.

The clinical feasibility of this approach was first examined in a phase I study, as described in Chapter 2. Nine patients, six lung cancer and three head and neck cancer patients, were injected with two consecutive doses of $60 \mathrm{MBq}{ }^{89} \mathrm{Zr}$-cetuximab or a single dose of $120 \mathrm{MBq}{ }^{89} \mathrm{Zr}$-cetuximab. No additional toxicity was associated with administration of ${ }^{89} \mathrm{Zr}$-cetuximab compared to standard cetuximab, and both presented dose schedules were considered safe. The higher ${ }^{89} \mathrm{Zr}$-cetuximab dose did not result in improved image contrast and, therefore, a dose of $60 \mathrm{MBq}$ was recommended for future studies.

The promising results of the phase I trial, inspired a larger phase II trial in locally advanced head and neck squamous cell carcinomas, as described in Chapter 3. A large variation in ${ }^{89} \mathrm{Zr}$-cetuximab uptake was observed between the 17 scanned patients. We 
found that the best contrast in the tumour was achieved at 6-7 days after injection of the PET tracer. Furthermore, it was shown that ${ }^{89} \mathrm{Zr}$-cetuximab PET provides additional information on the accessibility of the tumour, which cannot be extracted from EGFR expression levels or FDG PET/CT scans.

\section{Hypoxia and DCE-CT imaging for outcome prediction and treatment adaptation}

In Part 2 we used functional imaging for outcome prediction and treatment adaptation. In Chapter 4, the prognostic value of hypoxia HX4 PET/CT and dynamic contrast-enhanced CT (DCE-CT) were evaluated. We found that patients with a hypoxic tumour, as measured on the HX4 PET, had worse overall survival compared to patients with non-hypoxic tumours. The patients in this chapter received, in addition to standard (chemo)radiotherapy, the vasodilating drug nitroglycerin. Nitroglycerin was added to target and reduce tumour hypoxia. The differences on the scans at baseline and after administration of nitroglycerin revealed, however, a mixed response to nitroglycerin: both increases and decreases in hypoxia levels and tumour blood flow and blood volume were observed on HX4 PET and DCE-CT.

Several radiotherapy adaptation strategies, to selectively increase the radiotherapy dose in radioresistant areas, were compared in the planning study in Chapter 5. We found that selective boosting subvolumes based on FDG PET or hypoxia PET was feasible in non-small cell lung cancer patients, without increasing the dose to the organs at risk. The highest dose was achieved for the hypoxia-boosting plans. For most patients, boosting the high metabolic subvolume (determined on FDG PET), also resulted in boosting the hypoxic areas, although to a lower dose, but not vice versa.

\section{Combining multiparametric imaging characteristics}

In the third part of this thesis, different strategies were examined to combine multiple (functional) imaging modalities on a subtumour level. In Chapter 6, we created multiple models to predict hypoxia levels in non-small cell lung cancer. Hypoxia is an interesting target for personalized radiotherapy treatment, however, hypoxia PET imaging is not widely available, expensive, and time-consuming. We presented a methodology to create virtual hypoxia images, based on the more widely available FDG PET/CT and DCE-CT imaging.

The same multiparametric imaging data set, containing hypoxia HX4 PET/CT, DCE-CT and FDG PET/CT, was utilized in Chapter 7 to identify subregions with characteristics phenotypes and to relate these subregions to treatment outcome. Two different clustering steps were performed based on all available imaging. First, 
clustering voxels in larger tumour regions to reduce registration uncertainties and noise. Next, grouping these subregions of all patients into phenotypic clusters. We found that one of the clusters, with high hypoxia levels, high metabolism (FDG uptake), and intermediate tumour blood flow and blood volume, was related to a highrisk tumour type. Patients with a large volume of this cluster had significantly worse survival compared to patients with no, or only a small volume of this cluster.

\section{How to move forward?}

In Chapter 8, we discussed the opportunities and the challenges of the combined use of multiple imaging modalities. We distinguished two complementary strategies, a data-driven and biological-driven approach, to move forward with multiparametric imaging. Finally, in Chapter 9, we discussed the results presented in this thesis and the future perspectives of functional imaging in radiotherapy. We believe that the combination of very accurate radiation delivery modalities with biologically validated targets, will allow further optimization and personalization of treatment to increase tumour control and reduce normal tissue toxicities. 



\section{Samenvatting}

\section{(Dutch summary)}




\section{Achtergrond en doel van het proefschrift}

Kanker is een wereldwijd gezondheidsprobleem met meer dan 14 miljoen nieuwe diagnoses in 2012. Het wordt verwacht dat het aantal kankergevallen zal blijven toenemen tot bijna 24 miljoen nieuwe gevallen in 2035. Voor vele kankersoorten zijn de overlevingskansen van patiënten nog steeds gering, ondanks de substantiële verbeteringen van de behandelopties. De behandeling van kanker wordt bemoeilijkt doordat kankercellen erg lijken op gezonde cellen en door de heterogeniteit van de ziekte.

Om de prognose van patiënten te verbeteren en om onnodige toxiciteit en kosten te voorkomen, is het essentieel om zo snel mogelijk de meest geschikte behandeling te kiezen. Functionele medische beeldvorming kan de personalisatie van behandelingen verbeteren door patiënten te groeperen op basis van hun prognose en verwachte profijt van een bepaalde behandeling. Functionele beeldvorming maakt het mogelijk om de heterogeniteit van een tumor in kaart te brengen, resistente tumorgebieden te identificeren en adaptieve behandeling te ondersteunen.

In dit proefschrift is het gebruik van functionele beeldvormingstechnieken voor het karakteriseren van de tumor en tumor subvolumes verkend. Positronemissietomografie (PET) is gebruikt voor het visualiseren van een gelabeld medicijn, zuurstoftekort (hypoxie) en het metabolisme van de tumor. Dynamische contrast-versterkte computertomografie (DCE-CT) is gebruikt voor het analyseren van de tumor vasculatuur. Vervolgens zijn de biomarkers berekend op basis van deze beeldvormingstechnieken getest als potentiële prognostische markers, als markers voor patiënt stratificatie en voor het aanpassen van de radiotherapie behandelplannen. Patiënten met niet-kleincellige longkanker en hoofd-halstumoren zijn in de studies geanalyseerd.

\section{Niet-invasieve beeldvorming van gelabelde medicijnen}

In het eerste deel van dit proefschrift is de tumoropname van monoklonale antilichamen gevisualiseerd en gekwantificeerd. Monoklonale antilichamen kunnen zich met erg hoge affiniteit en specificiteit binden aan een doelwit. Cetuximab is een voorbeeld van zo een antilichaam dat zich bindt aan de epidermale groeifactor receptor (EGFR). Het succes van een behandeling met monoklonale antilichamen is afhankelijk van de expressie van receptoren in de tumor en afhankelijk van hoe goed het medicijn de tumor kan bereiken. Wij hebben de bereikbaarheid van de tumor onderzocht met PET, door cetuximab te labellen met radioactief Zirconium-89.

De klinische haalbaarheid van deze methode is eerst onderzocht in een fase I studie, zoals beschreven in Hoofdstuk 2. Negen patiënten, zes longkanker en drie hoofd-halskanker patiënten, zijn geïnjecteerd met twee opeenvolgende doses van $60 \mathrm{MBq}{ }^{89} \mathrm{Zr}$-cetuximab of een enkele dosis van $120 \mathrm{MBq}{ }^{89} \mathrm{Zr}$-cetuximab. Geen extra 
toxiciteit werd geobserveerd na de toediening van ${ }^{89} \mathrm{Zr}$-cetuximab vergeleken met standaard cetuximab en beide dosis schema's zijn veilig bevonden. De hogere dosis ${ }^{89} \mathrm{Zr}$-cetuximab resulteerde niet in een verbeterd beeldcontrast en daarom wordt een dosis van $60 \mathrm{MBq}$ aangeraden voor toekomstige studies.

De veelbelovende resultaten van de fase I studie zijn de inspiratie geweest voor een grotere fase II studie in patiënten met lokaal gevorderde hoofd-halstumoren, zoals beschreven in Hoofdstuk 3. Een grote variatie in ${ }^{89} \mathrm{Zr}$-cetuximab opname werd geobserveerd tussen de 17 gescande patiënten. Het beste contrast in de tumor werd 6-7 dagen na injectie van de PET tracer bereikt. Daarnaast hebben we laten zien dat ${ }^{89} \mathrm{Zr}$-cetuximab PET extra informatie geeft die niet verkregen kan worden door EGFR expressie analyses of FDG PET/CT scans.

\section{Hypoxie en DCE-CT beeldvorming voor prognose bepaling en therapieadaptatie}

In deel 2 hebben we functionele beeldvormingstechnieken gebruikt voor het bepalen van de patiënt prognose en voor het aanpassen van radiotherapie behandelplannen. In Hoofdstuk 4 zijn de prognostische waarde van HX4 PET/CT en dynamische contrast-versterkte computertomografie (DCE-CT) onderzocht. We hebben gevonden dat patiënten met hypoxische tumoren, bepaald met HX4 PET, slechtere overlevingskansen hebben vergeleken met patiënten met niet-hypoxische tumoren. De patiënten onderzocht in dit hoofdstuk kregen naast de standaard chemo(radiotherapie) het vaatverwijdende middel nitroglycerine met als doel om hypoxie te verminderen. Het verschil tussen de scans met en zonder nitroglycerine liet echter gemixte resultaten zien: zowel toenames als afnames in hypoxie, perfusie en bloedvolume werden geobserveerd op de HX4 PET en DCE-CT scans.

Verschillende strategiën om selectief de bestralingsdosis in radioresistente gebieden te verhogen zijn vergeleken in Hoofdstuk 5. Selectieve dosis verhoging in subvolumes gebaseerd op FDG PET en hypoxie PET was haalbaar in niet-kleincellige longkanker patiënten, zonder de dosis in de omliggende organen te verhogen. De hoogste dosis kon behaald worden in de hypoxie plannen. Voor de meeste patiënten resulteerde het verhogen van de dosis in de metabolisch actieve gebieden (FDG PET opname) ook in een verhoging in de hypoxische gebieden, maar niet vice versa.

\section{Combineren van multiparametrische beeldkarakteristieken}

In het derde deel van dit proefschrift zijn verschillende manieren onderzocht om multiparametrische beeldkarakteristieken op een subtumor niveau te combineren. In Hoofdstuk 6 hebben we verschillende modellen gemaakt om hypoxie te voorspellen 
in niet-kleincellige longkanker. Hypoxie is een interessant doelwit voor gepersonaliseerde radiotherapie. Hypoxie PET is echter niet overal beschikbaar, duur en tijdrovend. Wij hebben een methodologie ontwikkeld om virtuele hypoxie beelden te reconstrueren op basis van de meer gangbare beeldvormingstechnieken FDG PET/CT en DCE-CT.

Dezelfde multiparametrische dataset, bestaande uit hypoxie HX4 PET/CT, DCECT en FDG PET/CT, is in Hoofdstuk $\mathbf{7}$ gebruikt voor het identificeren van tumor subvolumes met karakteristieke fenotypes en om deze te linken aan de prognose van de patiënten. Twee opeenvolgende clustering stappen zijn uitgevoerd op de beschikbare data. Eerst zijn de voxels in grotere tumorregio's gegroepeerd om beeldregistratie onzekerheden en ruis te verminderen. Vervolgens zijn deze subregio's van alle patiënten gegroepeerd in fenotypische clusters. Eén van deze clusters, gekenmerkt door zuurstoftekort (hoge HX4 PET opname), een hoog metabolisme (hoge FDG PET opname) en een gemiddelde perfusie en bloedvolume, correleerde met een hoog-risico tumor type. Patiënten met een groot (relatief) volume van dit cluster hadden significant slechtere overlevingskansen vergeleken met patiënten zonder dit cluster of met alleen een klein volume van het cluster.

\section{Toekomstperspectieven}

In Hoofdstuk 8 zijn de kansen en uitdagingen van het combineren van verschillende beeldvormende technieken beschreven. Twee strategiën zijn beschreven voor de toekomst van multiparametrische medische beeldvorming: een datagestuurde en een biologiegestuurde strategie. In Hoofdstuk 9 zijn tenslotte de resultaten van dit proefschrift en de toekomstperspectieven van functionele beeldvorming in radiotherapie beschreven. De combinatie van precieze bestralingstechnieken en gevalideerde biologische targets wordt verwacht bij te dragen aan de verdere optimalisatie en personalisatie van radiotherapie om zodoende locale tumorcontrole te verbeteren en de toxiciteit in normale weefsels te verminderen. 

Valorization addendum 


\section{Social relevance and target groups}

Cancer is one of the leading causes of mortality and morbidity worldwide. For many cancer types, the survival remains poor despite substantial advances in treatment options. In this thesis, we examined the tumour biology with different functional imaging modalities. A better understanding of the tumour biology and the tumour characteristics related to treatment resistance could improve current treatment options and help the development of new therapies.

To achieve the best treatment results, it is crucial to select the treatment with the highest expected benefit as quick as possible. Therapy could be adapted based on the patient prognosis. For example, treatment could be intensified in patients with poor prognosis, while in patients with a good prognosis, de-escalation of therapy dose could be considered. We used biomarkers derived from several functional imaging modalities to stratify patients based on their expected prognosis and to evaluate the expected treatment benefit. We showed that functional imaging could be used to redistribute the dose in the tumour and sculpt the dose for a specific case to better target the most resistant areas.

The ultimate aim of treatment personalization is to include the preference of the patient in the treatment decision; to balance increasing local tumour control and limiting toxicities to the surrounding healthy tissues based on the patient preference. Personalization will first of all affect the patient, their family, and the physician. In addition, it will befit the general population since cancer is a worldwide health problem. Improving treatment options will economically benefit the whole society.

\section{Activities and products}

A subset of the images acquired for the research presented in this thesis is publicly available (www.cancerdata.org). ${ }^{89} \mathrm{Zr}$-cetuximab PET/CT images of head and neck cancer patients, and HX4 PET/CT and FDG PET/CT images of non-small cell lung cancer patients are provided. The treatment plans generated for the planning study of Chapter 5 are also available on the same website. Researchers and other interested parties are free to reuse the images for their own research questions.

The methodologies used in Chapter 6 en Chapter 7 are largely based on opensource software. The performed image registration, the generation of the supervoxels, and the clustering of the supervoxels were all performed using freely available software. The methodology described in these chapters to combine multiparamteric images can easily be implemented and reused in other projects.

Functional imaging could have an important role in the further development of prediction models. Prediction models are being developed to support physicians in clinical decision-making. With the vast amount of information available for every 
patient, it becomes increasingly difficult for a physician to consider and incorporate all this information. Prediction models can combine data of multiple sources to predict patient prognosis. Some examples from the Maastro clinic research group are already available on www.predictcancer.org. Functional imaging, e.g. HX4 PET/CT imaging, has the potential to further improve these models and better support the physicians. Eventually, these prediction models could be integrated in decision support systems to help the patient in selecting a personalized treatment.

\section{Innovation}

Although the research presented in this thesis is based on a limited number of patients, the data sets are unique and difficult to acquire. To our knowledge, there is only one other clinical trial using ${ }^{89} \mathrm{Zr}$-cetuximab PET images. In that trial, ten patients with advanced colorectal cancer were included. Our studies are the first to visualize and quantify ${ }^{89}$ Zr-cetuximab PET uptake in head and neck cancer patients.

The combination of multiple functional images, as presented in Chapter 6 and Chapter 7, provides a unique insight in multiple tumour characteristics at the same time. With the increasing amount of data available, analysis methods are required that can manage multiple scans and can combine the knowledge. In Chapter 7 we provided an example of such a workflow.

In Chapter 4, we showed the prognostic value of hypoxia HX4 PET/CT imaging in non-small cell lung cancer. Although hypoxia PET imaging has shown to be prognostic before in several tumour types, this is the first study that shows the prognostic value of the novel hypoxia PET tracer HX4.

\section{Schedule and implementation}

The benefit of boosting specific radioresistant parts of the tumour is still under clinical evaluation. Maastro clinic is participating in a prospective phase II trial in non-small cell lung cancer (NCT01024829). Several other studies are ongoing, as discussed in the general discussion of this thesis. The results of these clinical trials will give more insight into the potential of such a strategy.

The prognostic value of the hypoxia PET marker HX4, needs to be further validated in a larger patient cohort. After validation, it can be implemented in prediction models. The prediction of hypoxia based on other imaging modalities (CT, FDG PET and DCECT) as presented in Chapter 6, could serve as a an alternative to hypoxia PET that is easier to implement in a clinical workflow.

Combining multiple imaging data sets will become proportionally important with the increasing amount of data available. The workflows presented in this thesis are based on several open-source packages and could be directly applied to other data. 
Acknowledgments

(Dankwoord) 
"Soms denk ik uren na en heb ik nog niks op papier, een andere keer bereik ik precies datzelfde in vijf minuten."

— Herman Finkers

$\mathrm{Na}$ iets meer dan vier jaar zwoegen en zweten is het geweldig leuk om alles samen te zien komen en terug te kunnen blikken op een paar mooie jaren. Mijn naam mag dan wel voorop dit boekje staan - en daar ben ik erg trots op - onderzoek doe je niet alleen en er zijn veel mensen die ik mag bedanken voor dit resultaat, voor de fijne samenwerking, hun hulp en support.

Om te beginnen gaat mijn dank uit naar mijn promotor, prof.dr. Philippe Lambin, en co-promotor, dr. ir. Wouter van Elmpt. Beste Philippe, bedankt dat ik deel mocht uitmaken van jouw onderzoeksgroep. Je zit boordevol ideeën en bent altijd opzoek naar nieuwe mogelijkheden. Je weet je studenten te stimuleren om dit ook te doen en breder te oriënteren dan alleen het directe onderwerp. Mijn promotie is hierdoor een erg divers leertraject geworden met veel verschillende soorten cursussen, congressen en leermomenten, bedankt. Beste Wouter, jij bent mijn grote vraagbaak geweest tijdens mijn promotie. Ik realiseer me meer en meer hoeveel geluk ik heb gehad dat je me vanaf het begin hebt begeleid met het plannen en aanpakken van zo'n groot project. Je grote hoeveelheid kennis, doelgerichtheid en no-bullshit houding maken het erg fijn om met je samen te werken. Ik waardeer het dat je voor iedereen altijd tijd vrij maakt (ook voor diegenen die je misschien officieel niet begeleidt).

Graag wil ik de leden van de beoordelingscommissie (prof. dr. F. Ramaekers, prof. dr. ir. A. Dekker, prof. dr. U. van der Heide, prof. dr. D. Thorwarth, prof. dr. ir. F. Verhaegen) bedanken voor het kritisch lezen en beoordelen van mijn thesis.

Het werk in dit proefschrift is voornamelijk gebaseerd op klinische studies. Dergelijke studies zijn enkel mogelijk door de bereidheid en het vertrouwen van patiënten om hier aan mee te werken. Bedankt voor alle waardevolle data die we dankzij jullie hebben kunnen verzamelen.

Het opzetten en uitvoeren van klinische studies is organisatorisch complex en vergt inspanningen op verschillende vlakken. Ik wil graag iedereen bedanken die hierbij betrokken is geweest: de patiëntenplanners, de trial poli (bedankt John en Claudia, jullie zijn van grote waarde voor alle klinische studies binnen Maastro), datamanagement (in het bijzonder Chantal, Anita en Rody), alle laboranten voor het maken van extra scans en plannen (vooral Judith bedankt voor al het werk dat je gedaan hebt voor de boost studie), het artsenteam, klinisch fysica (Michel, bedankt voor jouw hulp met de ${ }^{89} \mathrm{Zr}$-cetuximab PET en het delen van jouw PETkennis), ICT (Joeri en Ronald, ik had altijd lastige dataverzoeken, bedankt voor jullie ondersteuning). 
Deze thesis is niet alleen het resultaat van een fijne samenwerking tussen kliniek en research, maar ook onze collega's bij het Maastricht Universitair Medisch Centrum hebben ontzettend geholpen. Veel van de scans hebben we alleen kunnen maken dankzij de samenwerking met de afdeling Radiologie en Nucleaire Geneeskunde. Marco bedankt voor jouw input omtrent de verschillende CT-protocollen. Felix bedankt voor je waardevolle feedback. Véronique en Ernst-Jan van de afdeling Pathologie bedankt voor jullie hulp met de analyse van de EGFR-expressie.

De collega's van het VU medisch centrum, bedankt voor het delen van jullie Zirconium-89 PET imaging expertise.

Van het NKI wil ik graag Olga en Jolien bedanken voor het opzetten en uitvoeren van de ${ }^{89} \mathrm{Zr}$-cetuximab hoofd-hals studie. Ik heb onze samenwerking als erg prettig ervaren. Jan-Jakob en José, bedankt voor het delen van jullie ideeën over de longstudies. Matt, thank you for sharing your DCE-CT modelling code and help with setting it up.

Maastro lab: Ludwig and Ala, thank you for our discussions and sharing your views from a different perspective. Thank you to all the nice people I met from the lab: Kranthi, Marike, Nicolle, Tessa, Sanaz, Marijke, Carolien, Eloy, Venus and Simon.

Tonnis en André, bedankt voor de eerste kennismaking met de radiotherapie in Deventer. Jullie enthousiasme voor het vakgebied heeft me gemotiveerd om ook hierin verder te gaan en een promotietraject aan te gaan.

Graag wil ik alle coauteurs, voor zover nog niet genoemd, bedanken voor alle hulp en feedback. Judith en Frank, jullie hebben me in het begin van mijn promotie direct op weg geholpen met de ${ }^{89} \mathrm{Zr}$-cetuximab imaging studies, wat resulteerde in een voortvarende start. En al heeft de afronding wat langer op zich laten wachten, het resultaat mag er wezen. Bart, ik ga je hopelijk niet meer lastigvallen met weer een nieuwe analyse waardoor je resultaten weer moet aanpassen. Bedankt voor de samenwerking en dat je er altijd de humor wel van in kon zien. Esther en Dirk, bedankt voor het delen van jullie enorme kennis en enthousiasme.

Over the years, I had the pleasure to share our big research room with many many people. Your company made it always worth coming to the office. I will miss the coffee breaks with vlaai or treats from all over the world (I still have some yogurt balls for those who want...), our lunch walks and drinks. Thank you Patrick, Emmanuel, Georgi, Daniela, Fiere, Esther, Stefan, Lotte, Isabel, Sean, Adriana, Lucas, Daan, Davide, Celine, Pouya, Scott, Arthur, Bregtje, Pedro, Raghu, Mathieu, Abir, Joanna, Mariana, Ester, Qing, Johan, Matilde, Brent, Murillo, Jose, Ana, Sebastian, Yvonka, Abdalla, Alberto, Anshu, Cecile, Daniela, Frank, Henry, Inge, Jane, Leon, Jean, Scott, Leonard, Relinde, Simon, Tim, Turkey, Zhen, Zhenwei and Claire.

Karen, het was super om bij aankomst al een vriendelijk bekend gezicht te zien. Je hebt me snel op weg geholpen met het onderzoek, maar ook met de altijd gezellige 
Maastro feestjes. Je enthousiasme wist me, voordat ik überhaupt in dienst was, over te halen om mee te gaan naar het Oktoberfest. Skadi, je warme persoonlijkheid vormt een mooi contrast met je koude avonturen. Hopelijk komen we jou en Geert nog een keer tegen op zo'n mooi avontuur! Sara, we are already missing your bubbly personality in the office. It is not the same without your energy there. Shane, my favourite enemy, and secretly also good friend (my frienemy), you always manage to surprise me. Gabriel, you and Louise are very warm and welcoming people. I enjoyed having you as a roommate and later roommate of Mark, although Monte Carlo talk during Sunday morning breakfast was a bit much.. Ralph, enne? Ik heb door de jaren toch vooral je kruintje gezien, dat boven de monitor uitstak en heen en weer knikte. Bedankt dat je ons altijd pushte om even een frisse neus te halen. Evelyn, mijn lieve buurvrouw, bedankt dat je altijd alles in de gaten hield voor mij. Zonder jou had mijn leven waarschijnlijk nog minder structuur gehad. Janita, bedankt voor alle uurtjes club power, klimmen en squash. Het is leuk om te zien hoe fanatiek je altijd alles aanpakt. Timo, you are an easy person to like. I enjoyed our many discussions.

Mark, Jasper, Renee en Wouter, wat zijn we al op veel vette tripjes geweest en hebben we wazige dingen meegemaakt. Het is niet zo makkelijk om mensen te vinden die enthousiast worden van de meest onpraktische vastelaovend pekskes, eetbare paddenstoelen (geen zand in de mand!), en vakanties naar Transnistrië, Iran of Oekraïne. Maar het is gelukt! Ik kijk al uit naar het volgende avontuur!

Myrthe, ook al zien we elkaar niet al te vaak, het voelt altijd als vanouds. Super om zo'n loyale en gezellige vriendin te mogen hebben. Ik ben altijd weer positief verbaasd door je oprechtheid en eerlijkheid.

Dan zijn er nog heel veel mensen die ik in mijn studententijd in Enschede heb mogen leren kennen o.a. via mijn studentenhuis Los Chichis, onafhankelijk dispuut Xoun en handbalvereniging Cabezota. Het zijn te veel lieve mensen om op te noemen en ik ben bang iemand te vergeten als ik het wel probeer. Bedankt voor de gezellige momenten die we ook de laatste paar jaren hebben gehad, in Maastricht, of andere delen van het land.

Mijn paranimfen, Ruben en Jurgen, Jurgen en Ruben, ik vind het een hele eer dat jullie dit traject samen met mij willen afsluiten. Jurgen, ik kan nog heel goed herinneren dat we op onze eerste werkdag elkaar tegenkwamen bij ICT om ons laptopje op te halen. Wat zijn die jaren voorbijgevlogen! Je vrolijke karakter, Belgische trekjes en slechte grappen ga ik zeker missen. Wat hebben we toch gelachen in Aarhus. Why do ants never get sick? Ruben, of het nu sporten, skiën, borrelen, late avonden doorwerken aan een $\mathrm{PhD}$ filmpje of stressen voor een deadline is, ik heb mijn maatje gevonden! Ik houd vele mooie herinneringen over aan mijn jaren in Maastricht en hoop dat we elkaar zullen blijven zien.

Mams, Nico en Jurian, bedankt voor alle steun en bedankt dat ik altijd mijn eigen 
weg heb kunnen gaan. Mam je bent een waar voorbeeld voor mij. Je hebt me laten zien hoe je sterk, onafhankelijk en kritisch kunt zijn, maar vooral hoe je, wat er ook gebeurt, positief in het leven kunt staan. En dat alles met de nodige humor en het respect voor anderen. Ik ben blij dat je Nico hebt gevonden om de mooie momenten mee te delen.

Mark, it is quite unique to have someone by your side that truly understands what you're doing and what you're going through. You helped me with the research by always asking the right (annoying) questions. But way more importantly, you make life more interesting. I can completely be myself with you and I know that you will always support me. Our adventure together has just begun and I can't wait to see where it will bring us! Go raibh míle maith agaibh! 
Curriculum vitae 
Aniek Even was born on the $24^{\text {th }}$ of January 1988 in Losser, the Netherlands. After finishing her secondary education at Twents Carmel College Lyceumstraat in Oldenzaal in 2006, she enrolled in the Biomedical Engineering program of the University of Twente to combine her interests in both technical problemsolving and the complexity of the human body. During the Master's program, she had the opportunity to visit the Robarts Research Institute in London, Ontario, Canada. Here she performed an accuracy

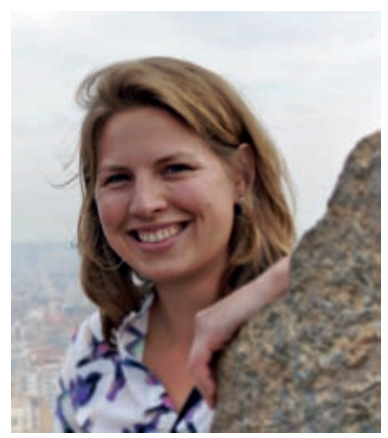
assessment of a 3D ultrasound imaging system for the thermal ablation of liver tumours. For her Master's thesis, she spent nine months at the Radiotherapeutic Institute RISO, Deventer, where she studied the optimization of high-dose-rate brachytherapy for prostate cancer. During this project she worked with 3D transrectal ultrasound and in-room cone-beam CT. In 2013 she obtained her Master's degree. In September of the same year, she joined the research team of Maastro clinic, Maastricht. As a $\mathrm{PhD}$ candidate she studied a wide range of functional imaging modalities (a variety of PET tracers, dynamic contrast-enhanced CT, dualenergy CT) to characterize the tumour and tumour subvolumes for radiotherapy purposes. She was involved in several large (multicentre) radiotherapy trials in nonsmall cell lung cancer and head and neck cancer.

\section{Grants and awards}

- BIGART travel grant, Biology-Guided Adaptive Radiotherapy, 2017, Aarhus, Denmark.

- Poster award, best clinical poster, international symposium Quantification and Standardisation in Medial and Preclinical Imaging: state of the art and future developments, 2017, Groningen, the Netherlands.

- ARTFORCE/ESTRO travel grant, Incorporating imaging in radiation oncology treatment delivery, 2015, Barcelona, Spain.

- EMIM travel grant, European Molecular Imaging Meeting, 2014 Antwerp, Belgium.

- ARTFORCE/ESTRO travel grant, Basic Clinical Radiobiology, 2014, Istanbul, Turkey. 


\section{Scientific publications}

- Ureba, A., Lindblom. E., Dasu, A., Uhrdin, J., Even, A. J. G., van Elmpt, W., Lambin, P., Wersäll, P. \& Toma-Dasu, I. Non-linear conversion of HX4 uptake for automatic segmentation of hypoxic volumes and dose prescription. Acta Oncol. 2017. Article in Press.

- Even, A. J. G., Reymen, B., La Fontaine, M. D., Das, M., Mottaghy, F. M., Belderbos, J. S. A., De Ruysscher, D., Lambin, P. \& van Elmpt, W. Clustering of multi-parametric functional imaging to identify high-risk subvolumes in nonsmall cell lung cancer. Radiother Oncol. 2017. Advance online publication; DOI: 10.1016/j.radonc.2017.09.041.

- Even, A. J. G., Reymen, B., La Fontaine, M. D., Das, M., Jochems, A., Mottaghy, F. M., Belderbos, J. S. A., De Ruysscher, D., Lambin, P. \& van Elmpt, W. Predicting tumor hypoxia in non-small cell lung cancer by combining CT, FDG PET and dynamic contrast-enhanced CT. Acta Oncol. 2017. Advance online publication; DOI: $10.1080 / 0284186 X .2017 .1349332$.

- Lambin, P., Leijenaar, R. T. H., Deist, T. M., Peerlings, J., de Jong, E. E. C., van Timmeren, J., Sanduleanu, S., Larue, R. T. H. M., Even, A. J. G., Jochems, A., van Wijk, Y., Woodruff, H., van Soest, J., Lustberg, T., Roelofs, E., van Elmpt, W., Dekker, A., Mottaghy, F. M., Wildberger, J. E. \& Walsh, S. Radiomics: the bridge between medical imaging and personalized medicine. Nat Rev Clin Oncol. 2017. Advance online publication; DOI: 10.1038/nrclinonc.2017.141.

- Van Loon, J. ${ }^{*}$ Even, A. J. G. ${ }^{*}$, Aerts, H. J. W. L., Öllers, M., Hoebers, F., van Elmpt, W., Dubois, L., Dingemans, A. M. C., Lalisang, R. I., Kempers, P., Brans, B., Winnepenninckx, V., Speel, E. J., Thunnissen, E., Smits, K. M., Boellaard, R., Vugts, D. J., De Ruysscher, D. \& Lambin, P. PET imaging of zirconium-89 labelled cetuximab: A phase I trial in patients with head and neck and lung cancer. Radiother Oncol. 2017;122(2):267-73.

- Even, A. J. G. , , Hamming-Vrieze, O.", van Elmpt, W., Winnepenninckx, V. J. L., Heukelom, J., Tesselaar, M. E. T., Vogel, W. V., Hoeben, A., Zegers, C. M. L., Vugts, D. J., van Dongen, G. A. M. S., Bartelink, H., Mottaghy, F. M., Hoebers, F. \& Lambin, P. Quantitative assessment of Zirconium-89 labeled cetuximab using PET/CT imaging in patients with advanced head and neck cancer: a theragnostic approach. Oncotarget. 2017;8(3):3870-80. 
- Lindblom, E., Dasu, A., Uhrdin, J., Even, A. J. G., van Elmpt, W., Lambin, P., Wersäll, P. \& Toma-Dasu, I. Defining the hypoxic target volume based on positron emission tomography for image guided radiotherapy - the influence of the choice of the reference region and conversion function. Acta Oncol. 2017;56(6):819-25.

- Lambin, P., Zindler, J., Vanneste, B. G. L., van de Voorde, L., Eekers, D., Compter, I., Panth, K. M., Peerlings, J., Larue, R. T. H. M., Deist, T. M., Jochems, A., Lustberg, T., van Soest, J., de Jong, E. E. C., Even, A. J. G., Reymen, B., Rekers, N., van Gisbergen, M., Roelofs, E., Carvalho, S., Leijenaar, R. T. H., Zegers, C. M. L., Jacobs, M., van Timmeren, J., Brouwers, P., Lal, J. A., Dubois, L., Yaromina, A., Van Limbergen, E. J., Berbee, M., van Elmpt, W., Oberije, C., Ramaekers, B., Dekker, A., Boersma, L. J., Hoebers, F., Smits, K. M., Berlanga, A. J. \& Walsh, S. Decision support systems for personalized and participative radiation oncology. Adv Drug Deliv Rev. 2017;109:131-53.

- Even, A. J. G., De Ruysscher, D. \& van Elmpt, W. The promise of multiparametric imaging in oncology: how do we move forward? Eur J Nucl Med Mol Imaging. 2016;43(7):1195-8.

- Van Elmpt, W., Zegers, C. M. L., Reymen, B., Even, A. J. G., Dingemans, A. M. C., Öllers M., Wildberger, J. E., Mottaghy, F. M., Das, M., Troost, E. G. C. \& Lambin, P. Multiparametric imaging of patient and tumour heterogeneity in non-small-cell lung cancer: quantification of tumour hypoxia, metabolism and perfusion. Eur J Nucl Med Mol Imaging. 2016;43(2):240-8.

- Lambin, P., Zindler, J., Vanneste, B., van de Voorde, L., Jacobs, M., Eekers, D., Peerlings, J., Reymen, B., Larue, R. T. H. M., Deist, T. M., de Jong, E. E. C., Even, A. J. G., Berlanga, A. J., Roelofs, E., Cheng, Q., Carvalho, S., Leijenaar, R. T. H., Zegers, C. M. L., van Limbergen, E., Berbee, M., van Elmpt, W., Oberije, C., Houben, R., Dekker, A., Boersma, L., Verhaegen, F., Bosmans, G., Hoebers, F., Smits, K. \& Walsh, S. Modern clinical research: How rapid learning health care and cohort multiple randomised clinical trials complement traditional evidence based medicine. Acta Oncol. 2015;54(9):1289-300.

- Even, A. J. G., van der Stoep, J., Zegers, C. M. L., Reymen, B., Troost, E. G. C., Lambin, P. \& van Elmpt, W. PET-based dose painting in non-small cell lung cancer: Comparing uniform dose escalation with boosting hypoxic and metabolically active sub-volumes. Radiother Oncol. 2015;116(2):281-6. 
- Zegers, C. M. L., van Elmpt, W., Reymen, B., Even, A. J. G., Troost, E. G. C., Öllers, M. C., Hoebers, F. J. P., Houben, R. M. A., Eriksson, J., Windhorst, A. D., Mottaghy, F. M., De Ruysscher, D. \& Lambin, P. In vivo quantification of hypoxic and metabolic status of NSCLC tumors using $\left[{ }^{18} \mathrm{~F}\right] \mathrm{HX} 4$ and $\left[{ }^{18} \mathrm{~F}\right] \mathrm{FDG}-\mathrm{PET} / \mathrm{CT}$ imaging. Clin Cancer Res. 2014;20(24):6389-97.

- Even, A. J. G., Nuver, T. T., Westendorp, H., Hoekstra, C. J., Slump, C. H. \& Minken, A. W. High-dose-rate prostate brachytherapy based on registered transrectal ultrasound and in-room cone-beam CT images. Brachytherapy. 2014;13(2):12836. 


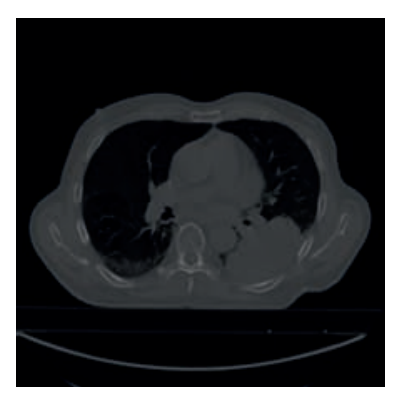

